\title{
Delirium as a disorder of brain network disintegration
}

Simone van Montfort 


\section{Delirium as a disorder of brain network disintegration}

Delirium als een stoornis van desintegratie van het hersennetwerk

(met een samenvatting in het Nederlands)

\section{Proefschrift}

ter verkrijging van de graad van doctor aan de

Universiteit Utrecht

op gezag van de

rector magnificus, prof.dr. H.R.B.M. Kummeling, ingevolge het besluit van het college voor promoties

in het openbaar te verdedigen op

\section{ISBN 978-94-6375-951-9}

For consistency some terms and graphical presentation of Tables and Figures have been standardized throughout this thesis. Therefore, there may be some differences with the articles that have been published.

\section{Copyright (C) 2020 Simone van Montfort}

All rights reserved. No part of this publication may be reproduced or transmitted in any form or by any means, electronic or mechanical, including photocopy, recording, or any information storage or retrieval system, without permission in writing from the author.

The copyright of articles that have been published or accepted for publication has been transferred to the respective journals.

Financial support for printing this thesis was kindly provided by the University Medical Center Utrecht Brain Center and by Alzheimer Nederland.

\section{Cover design Ramone de Bruin}

Lay out Camiel Lemmens | www.persoonlijkproefschrift.nl

Printing Ridderprint | www.ridderprint.nl dinsdag 22 september 2020 des ochtends te 11.00 uur

$$
\text { door }
$$

\section{Simone Jozefa Theodora van Montfort}

geboren op 24 november 1990

$$
\text { te Den Haag }
$$




\section{Promotoren:}

Prof. dr. A.J.C. Slooter

Prof. dr. J. Hendrikse

\section{Copromotor:}

Dr. E. van Dellen

\section{Table of contents}

Chapter 1 General introduction

Part I: Vulnerability for delirium

Chapter 2 Brain network disintegration as a final common pathway for delirium: a systematic review and

qualitative meta-analysis

Chapter 3 Predisposition for delirium and EEG characteristics

Chapter 4 fMRI network correlates of predisposing risk factors for delirium: a cross-sectional study

Part II: Clinical syndrome of delirium

Chapter 5 Resting-state $\mathrm{fMRI}$ reveals network disintegration during delirium

Chapter 6 Delirium is not associated with altered hub flexibility of the posterior

Part III: Longitudinal changes after delirium

Chapter 7 Functional brain network changes after major surgery and delirium

ary and general discussion

Chapter 8 Summary

Appendices

Dutch summary (Nederlandse samenvatting)

Acknowledgements (Dankwoord)

About the author

List of publications 


\section{Chapter 1}

General introduction 


\section{Delirium}

Delirium is derived from the Latin word 'delirare', which literally means 'a disturbed state of mind' and has been known since ancient times. Hippocrates (460 - 370 BC) may have been the first to describe a syndrome characterized by confusion and restlessness, which fluctuated over time and which was associated with physical illness. He called this condition 'phrenitis' 1,2. In the following eras, many other names have been used to describe the syndrome, including brain failure, acute change of mental status, delirium, acute brain dysfunction, intensive care psychosis and encephalopathy. Of these, the term delirium became the official name. In the 90's, Diagnostic and Statistical Manual of Mental Disorders (DSM) criteria were developed for the syndrome and delirium became an official diagnosis.

Currently, delirium is defined as a neuropsychiatric syndrome, characterized by an acute change in attention and awareness. It is always a direct consequence of an underlying medical condition ${ }^{3}$. It is a common syndrome, affecting $10-50 \%$ of the hospitalized elderly, and is a burden for patients and their relatives ${ }^{4}$. Delirium has several clinical manifestations, which are commonly divided into hypoactive, hyperactive and mixed subtypes. Hypoactive delirium is characterized by lethargy and lack of psychomotor behavior and speech. In contrast, the hyperactive subtype is defined by restlessness, hyper vigilance and agitation ${ }^{3}$. The mixed type manifest both hypoactive and hyperactive elements ${ }^{5}$. All types are associated with negative outcomes, such as prolonged hospital stay, long-term cognitive impairment and dementia ${ }^{4}$.

The development of delirium is usually the consequence of an interaction of various heterogeneous risk factors ${ }^{4,6,7}$. Risk factors for delirium can be divided in predisposing and precipitating factors ${ }^{6}$. Predisposing risk factors cover the baseline vulnerability to delirium, such as older age or cognitive impairments. Precipitating risk factors for delirium determine acute changes that can trigger the syndrome, such as surgery or sedation. In patients with predisposing risk factors for delirium, e.g. an elderly individual with some cognitive problems, a relatively mild precipitating factor, such as an uncomplicated cystitis, can lead to an episode of delirium ${ }^{4,6}$. Although heterogeneous various risk factors for delirium have been identified, the exact mechanism of how (a combination of) risk factors can lead to development of delirium is currently unknown ${ }^{4,6}$. In addition, the biological mechanism underlying the clinical syndrome of delirium is currently unknown. Nevertheless, recent studies have indicated that delirium can be accompanied with alterations in brain (network) activity ${ }^{8-10}$. Studying the brain network in relation to delirium may therefore give us new insights in this complex clinical syndrome.

\section{The brain as a complex network}

The brain can be considered as a complex network. On microscale, neurons are connected with each other via synapses. On macroscale, groups of thousands of neurons are forming functional modules between different brain areas, connected via grey and white matter tracts. The organization of the brain network is important for proper communication between different brain areas ${ }^{11,12}$. A distinction can be made between structural and functional networks. Structural networks can be seen as the roads that are connecting different brain areas, functional networks can be seen as the 'cars' that are driving these roads to exchange information between different brain areas. Structural networks are therefore generally more stable and functional networks have a more dynamical nature ${ }^{11}$. Understanding the organization of communication in the healthy brain and how this architecture is altered in the diseased brain, is an essential aspect in neuroscience. Network science is increasingly used to evaluate this brain communication ${ }^{13-15}$

Network science is a mathematical approach that gives an abstract representation of the elements of a system and their interactions, by dividing the network into nodes and connections between the nodes, i.e. the edges ${ }^{16}$. It is possible to map these brain networks with neuroimaging techniques such as (functional) magnetic resonance imaging (MRI). This method uses strong magnetic fields, magnetic field gradients, and radio waves to generate images of the brain and to measure brain activity by detecting changes associated with blood flow ${ }^{17}$. Another method to map these networks is by using neurophysiological measurements, such as electroencephalography (EEG) ${ }^{18}$, i.e. an electrophysiological monitoring 
method to record electrical activity of the brain (Figure 1). As the network is constructed, several network characteristics can be calculated, such as global connectivity strength, network efficiency and network integration. Connectivity strength represents the mean strength of the connections of the network. Network efficiency is a measure of how efficiently information is transferred from one side of the network to the other (Figure 2). Network integration is a measure of how central the network is organized (Figure 2).

Brain network organization differs between individuals or groups 11,19 Specific brain network alterations can be considered as a characteristic of a specific disorder or a marker for vulnerability, such as in epilepsy ${ }^{20-22}$. As brain network organization is fundamentally related to cognitive functioning and network alterations have been found during the acute state of delirium ${ }^{8-10,23}$, brain network disintegration may play a role in the underlying mechanism of the clinical syndrome.

\section{Objectives}

The aim of this dissertation is to evaluate delirium as a disorder of brain network disintegration. Network disintegration was tested as biological substrate of:

A. Vulnerability for delirium

B. Clinical syndrome of delirium

C. Longitudinal changes after delirium

\section{Outline of this dissertation}

Part I of this dissertation is focused on network disintegration and vulnerability for delirium. Chapter $\mathbf{2}$ is a systematic review and quantitative meta-analysis, in which we tested the hypothesis that delirium and its risk factors are associated with consistent brain network changes. In chapter 3 the relation between predisposition for delirium and delirium-related EEC characteristics is studied. In chapter $\mathbf{4}$, the relation between deliriumrelated $\mathrm{fMRI}$ network characteristics and predisposing risk for delirium is evaluated.
Part II of this dissertation is focused on the clinical symptoms of delirium and brain network disintegration. In chapter 5, fMRI network organization during delirium is studied to increase our understanding of the global organization of the functional network during the disorder, to localize possible alterations and to relate possible alterations to delirium severity. In chapter 6, the hypothesis is tested that delirium, with its fluctuating course, may rely on a more dynamical brain process.

Part III of this dissertation evaluates the hypothesis of lasting brain network disintegration due to delirium as the possible biological concept of negative outcomes, such as long-term cognitive impairment. In chapter 7, changes in the functional brain network over time, influenced by postoperative delirium, are studied.

In part IV, the main findings of this dissertation are summarized (chapter 8) and methodological considerations, suggestions for future research perspectives and possible clinical implications are discussed (chapter 9). 


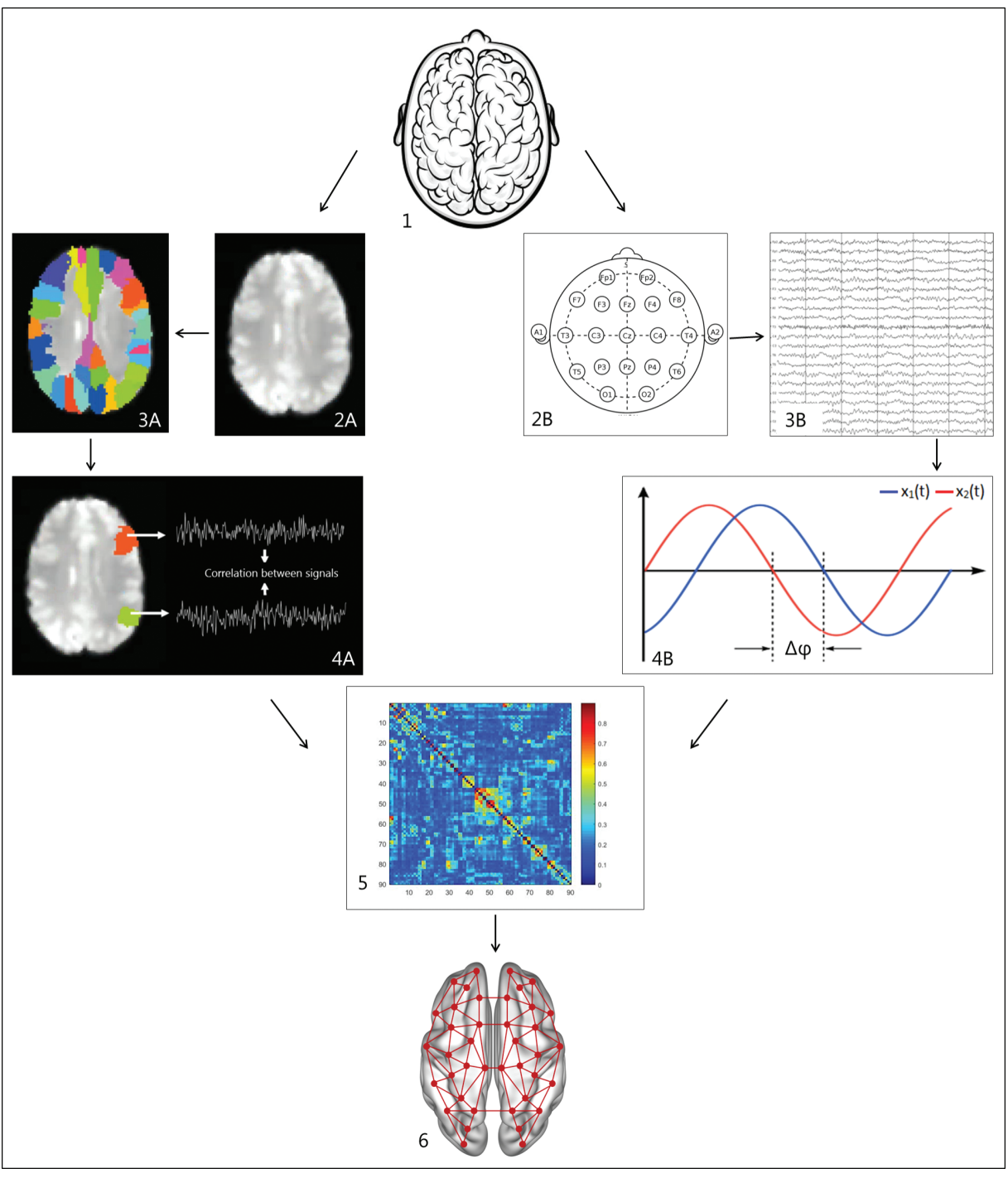

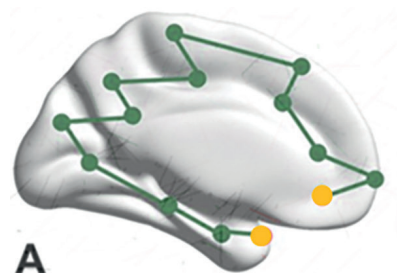

A

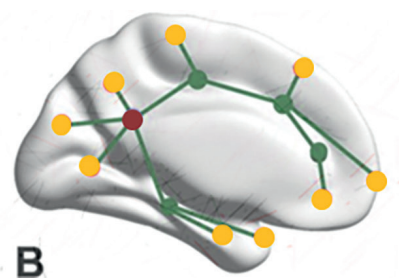
tween a less efficient and sparsely integrated network (A) and an highly efficient and highly integrated network (C). The length of the path between the two nodes that are furthest apart, can be used as a measure for network efficiency. The fraction of the yellow nodes, i.e. nodes that only have one connection, can be used as a measure of network integration.

Figure 1 Mapping the brain network by using neuroimaging or neurophysiological measurements. Neuroimaging or neurophysiological measurements can be used to obtain information from the brain (1). After neuroimaging, functional or structural brain images are available (2A). The brain is divided in different brain regions, using a predefined brain parcellation atlas (3A). Signals from different brain regions are coupled using the statistical interdependencies between their time series, for example by calculating the correlation coefficient between the time series from the brain regions (4A), resulting in a connectivity matrix (5). Using neurophysiological measurement, such as electroencephalography (EEG), electrodes are placed at predefined locations at the skull of a participant (2B). Electrodes record time series originating from functional brain signals (3B). Time series of different electrodes are coupled using the statistical interdependencies between time series, reflecting the strength of the coupling, for example by using the difference in phase of the time series (4B), resulting in a connectivity matrix (5). From this connectivity matrix, the brain network can be constructed (6) and brain network characteristics can be evaluated. 


\section{References}

1. Slooter A. Delirium, what's in a name? 2017. https://academic.oup.com/bja/articleabstract/119/2/283/4049136. Accessed April 15, 2020.

2. Morandi A, Pandharipande P, Trabucchi M, et al. Understanding international differences in terminology for delirium and other types of acute brain dysfunction in critically ill patients. Intensive Care Med. 2008;34(10):1907-1915. doi:10.1007/s00134008-1177-6

3. American Psychiatric Association. Diagnostic and Statistical Manual of Mental Disorders (5th Ed.). Washington, DC; 2013.

4. Marcantonio ER. Delirium in Hospitalized Older Adults. Solomon CG, ed. N EnglJ Med. 2017;377(15):1456-1466. doi:10.1056/NEJMcp1605501

5. Yang FM, Marcantonio ER, Inouye SK, et al. Phenomenological Subtypes of Delirium in Older Persons: Patterns, Prevalence, and Prognosis. Psychosomatics. 2009;50(3):248 254. doi:10.1176/APPI.PSY.50.3.248

6. Inouye SK, Westendorp RG, Saczynski JS. Delirium in elderly people. Lancet. 2014;383(9920):911-922. doi:10.1016/S0140-6736(13)60688-1

7. Zaal IJ, Devlin JW, Peelen LM, Slooter AJC. A Systematic Review of Risk Factors for Delirium in the ICU*. Crit Care Med. 2015;43(1):40-47. doi:10.1097/ CCM.0000000000000625

8. van Dellen E, van der Kooi AW, Numan T, et al. Decreased Functional Connectivity and Disturbed Directionality of Information Flow in the Electroencephalography of Intensive Care Unit Patients with Delirium after Cardiac Surgery. Anesthesiology. 2014;121(2):328-335. doi:10.1097/ALN.0000000000000329

9. Numan T, Slooter AJC, van der Kooi AW, et al. Functional connectivity and network analysis during hypoactive delirium and recovery from anesthesia. Clin Neurophysiol. 2017;128(6):914-924. doi:10.1016/j.clinph.2017.02.022

10. Choi SH, Lee H, Chung TS, et al. Neural network functional connectivity during and after an episode of delirium. Am J Psychiatry. 2012;169(5):498-507. doi:10.1176/appi. ajp.2012.11060976

11. Bullmore E, Sporns O. Complex brain networks: Graph theoretical analysis of structural and functional systems. Nat Rev Neurosci. 2009;10(3):186-198. doi:10.1038/nrn2575

12. Stam CJ. Modern network science of neurological disorders. Nat Rev Neurosci. 2014;15(10):683-695. doi:10.1038/nrn3801

13. Bassett DS, Sporns O. Network neuroscience. Nat Neurosci. 2017;20(3):353-364. doi:10.1038/nn.4502
14. Sporns O, Zalesky A. An Introduction to Brain Networks. In: Fundamentals of Human Imaging Connectomics. Elsevier; 2016:1-35. doi:10.1016/B978-0-12-407908-3.00001-7

15. van Straaten ECW, Stam CJ. Structure out of chaos: Functional brain network analysis with EEG, MEG, and functional MRI. Eur Neuropsychopharmacol. 2013;23(1):7-18. doi:10.1016/j.euroneuro.2012.10.010

16. Biggs N, Lloyd EK, Wilson RJ. Graph Theory, 1736-1936. Oxford: Clarendon Press; 1986. doi:10.1086/352170

17. van den Heuvel MP, Hulshoff Pol HE. Exploring the brain network: a review on restingstate fMRI functional connectivity. Eur Neuropsychopharmacol. 2010;20(8):519-534. doi:10.1016/j.euroneuro.2010.03.008

18. Stam CJ, Reijneveld JC. Graph theoretical analysis of complex networks in the brain Nonlinear Biomed Phys. 2007;1(1):3. doi:10.1186/1753-4631-1-3

19. Stam CJ, van Straaten ECW. The organization of physiological brain networks. Clin Neurophysiol. 2012;123(6):1067-1087. doi:10.1016/j.clinph.2012.01.011

20. Douw L, van Dellen E, de Groot M, et al. Epilepsy is related to theta band brain connectivity and network topology in brain tumor patients. BMC Neurosci. 2010;11(1):103. doi:10.1186/1471-2202-11-103

21. van Diessen E, Diederen SJH, Braun KPJ, Jansen FE, Stam CJ. Functional and structura brain networks in epilepsy: What have we learned? Epilepsia. 2013;54(11):1855-1865. doi:10.1111/epi.12350

22. Crossley NA, Mechelli A, Scott J, et al. The hubs of the human connectome are generally implicated in the anatomy of brain disorders. Brain. 2014;137(8):2382-2395. doi:10.1093/brain/awu132

23. Sporns $\mathrm{O}$. Contributions and challenges for network models in cognitive neuroscience. Nat Neurosci. 2014;17(5):652-660. doi:10.1038/nn.3690 


\section{Part 1}

Vulnerability for delirium 


\section{Chapter 2}

Brain network disintegration as a final common pathway for delirium: a systematic review and qualitative meta-analysis

Published in Neurolmage Clinical 2019; 23: 101809

Simone JT van Montfort

Edwin van Dellen

Cornelis J Stam

Amtul Ahmad

Lara J Mentink

Carla W Kraan

Andrew Zalesky

Arjen JC Slooter 


\section{Abstract}

Delirium is an acute neuropsychiatric syndrome characterized by altered levels of attention and awareness with cognitive deficits. It is most prevalent in elderly hospitalized patients and related to poor outcomes. Predisposing risk factors, such as older age, determine the baseline vulnerability for delirium, while precipitating factors, such as use of sedatives, trigger the syndrome. Risk factors are heterogeneous and the underlying biological mechanisms leading to vulnerability for delirium are poorly understood. We tested the hypothesis that delirium and its risk factors are associated with consistent brain network changes. We performed a systematic review and qualitative meta-analysis and included 126 brain network publications on delirium and its risk factors. Findings were evaluated after an assessment of methodological quality, providing $\mathrm{N}=99$ studies of good or excellent quality on predisposing risk factors, $\mathrm{N}=10$ on precipitation risk factors and $\mathrm{N}=7$ on delirium. Delirium was consistently associated with functional network disruptions, including lower EEG connectivity strength and decreased fMRI network integration. Risk factors for delirium were associated with lower structural connectivity strength and less efficient structural network organization. Decreased connectivity strength and efficiency appear to characterize structural brain networks of patients at risk for delirium, possibly impairing the functional network, while functional network disintegration seems to be a final common pathway for the syndrome.

\section{Introduction}

Brain network organization is fundamentally related to cognitive functioning ${ }^{1}$ and disturbed in various neurological and psychiatric disorders ${ }^{2}$. These impairments can even be a fingerprint of a specific disorder ${ }^{3}$ or a marker for vulnerability ${ }^{4,5}$. Delirium is an acute neuropsychiatric syndrome characterized by an altered level of attention and awareness with other cognitive deficits, due to another medical condition ${ }^{6}$. It has several clinica manifestations: hypoactive, hyperactive and a mixed type. Hypoactive delirium is characterized by lethargy and lack of psychomotor behavior and speech. Patients with the hyperactive subtype, however, demonstrate features of restlessness, hyper vigilance and agitation, and often experience hallucinations and delusions. The mixed type manifest both hypoactive and hyperactive elements ${ }^{7}$. Delirium is a common and serious clinical complication, affecting $10-50 \%$ of hospitalized elderly patients and related to poor outcomes, such as long-term cognitive impairment and death ${ }^{8}$ Delirium has been hypothesized to be a disconnection syndrome, caused by breakdown of brain networks ${ }^{9-11}$

Several risk factors for delirium have been recognized. However, known risk factors are heterogeneous and the underlying biological mechanisms leading to vulnerability for delirium are poorly understood. Risk factors for delirium can be distinguished into predisposing and precipitating factors ${ }^{12}$. Predisposing risk factors determine the baseline vulnerability for delirium, for example due to older age or cognitive impairment. Precipitating risk factors are acute changes that trigger the syndrome, for example sedation. Here, we evaluate if various predisposing risk factors induce similar brain network alterations, creating a more vulnerable (i.e. less connected and/ or less integrated) brain network. Network vulnerability may lower the threshold for a transition from a healthy state towards disturbed brain activity and connectivity. Precipitating factors may then cause an acute alteration in brain dynamics, that results in a global loss of functional brain interactions as a final common pathway to delirium.

Graph theory provides tools to quantitatively analyze network organization from a whole brain perspective. A graph represents a network of nodes and 
connections between the nodes, i.e. the edges. On a macro level, structura brain networks can be reconstructed using anatomically defined regions as nodes and white matter tracts connecting these brain regions as edges. It is possible to map these brain networks with neuroimaging techniques such as magnetic resonance imaging (MRI) with diffusion tensor imaging (DTI). The communication between brain regions (i.e. statistical relations or synchronization between time series of neural activity, recorded from different brain areas) is regarded as functional connectivity, which can be used to reconstruct a functional network. These functional brain networks can be characterized with imaging techniques such as functional MRI (fMRI) and positron emission tomography (PET), but also using neurophysiological measurements, such as near infrared spectroscopy (NIRS), magnetoencephalography (MEG) and electroencephalography (EEG) ${ }^{13}$. In the latter case, nodes are the electrodes of the EEG recording and synchronized activities between brain regions are considered as edges. The EEG signal consists of different oscillations, i.e. delta $(0.5-4 \mathrm{~Hz})$, theta $(4-8 \mathrm{~Hz})$, alpha $(8-13 \mathrm{~Hz})$, beta $(13-30 \mathrm{~Hz})$, and gamma $(>30 \mathrm{~Hz})$ band. All frequency bands show different functional network characteristics and can be analyzed separately. When the edges in a network are binary, i.e. a threshold is used to define if a connection is either present or absent, this is called an unweighted network ${ }^{14}$. A weighted network by contrast takes the connectivity strength or the weight of an edge into account ${ }^{14}$. Once a brain network is reconstructed, measures from graph theory can be used to further characterize network organization, which is illustrated in Supplementary Information Figure S1.

The aim of this investigation was to compare graph theoretical studies on delirium and its risk factors to test the hypothesis that a disturbance in network organization is a final common pathway in the pathophysiology of delirium. The inclusion of risk factors was based on a recent landmark review on delirium ${ }^{12}$.

\section{Methods}

\section{Systematic review}

Risk factors for delirium

In this systematic review and qualitative meta-analysis, we based the inclusion of risk factors on a recent landmark review that described 29 recognized risk factors for delirium ${ }^{12}$. Dementia; cognitive impairment, i.e. cognitive problems without the clinical diagnosis of dementia; history of delirium; functional impairment; visual impairment; hearing impairment; comorbidity; severity of illness; depression; history of transient ischemic attack or stroke; alcohol misuse; and older age were considered as predisposing risk factors for delirium ${ }^{12}$. Polypharmacy; psychoactive drugs; use of sedatives or hypnotics; use of physical restraints; use of bladder catheter; acute kidney injury; decreased serum albumin; decreased sodium; hypoglycemia; hypokalemia; metabolic acidosis; infection; iatrogenic disease; surgery; recent trauma; urgent admission; and previous coma were considered as precipitating risk factors for delirium ${ }^{12}$.

Delirium

As delirium is regarded as a manifestation of encephalopathy ${ }^{15-17}$, we included articles on either term, and grouped these into one category denoted as 'delirium'.

\section{Network outcomes}

Since graph theory studies may include a variety of outcomes, we focused on the outcomes that are more commonly analyzed and have a straightforward interpretation, i.e. (connectivity) strength, global efficiency, local clustering and modularity (Supplementary Information Figure S1).

\section{Search term and search strategy}

References for the systematic review were identified through searches of PubMed and EMBASE from inception to September 2018, by use of relevant terms "connectivity", "network", "graph", "disconnection", "dementia", "cognitive impairment", "history of delirium", "functional impairment", "visual impairment", "hearing impairment", "comorbidity or severity of illness", "depression", "(history of) transient ischemic 
attack or stroke", "alcohol misuse", "aging", "polypharmacy", "psychoactive drugs", "sedatives or hypnotics", "physical restraints", "bladder catheter", "acute kidney injury", "altered serum albumin level",

"altered sodium, glucose or potassium level", "metabolic acidosis", "infection", "iatrogenic disease", "surgery", "trauma admission", "urgent admission", "coma", "delirium", "encephalopathy", "magnetic resonance imaging", "electroencephalography", "electrocorticography", "diffusion tensor imaging", "resting state", "magnetoencephalography", "brain", "neuroimaging", "functional neuroimaging", "positron-emission tomography", "staining", "neurophysiology", "diffusion tractography", "diffusion magnetic resonance imaging", and "near infrared spectroscopy" (for the exact search term see Supplementary Information Tables S1 + S2). Articles resulting from these searches and relevant references cited in those publications were reviewed on the relevance of the title and the abstract by two authors (SVM and AA). The full text of potentially relevant articles were evaluated by two authors (SVM and AA).

Inclusion criteria

We included articles (a) published in English, (b) assessing whole brain graph analysis, (c) in humans (d) during delirium or during a state that is considered to be risk factor, (e) with use of a control group, ( $f$ ) for functional imaging with measurements conducted during resting state without intervention, and $(\mathrm{g})$ assessing one or more of the following outcomes: (normalized) connectivity strength of the global network, (normalized) global efficiency or (normalized) path length of the global network, (normalized) local clustering of the global network, and/or (normalized) modularity of the global network (Supplementary Information Figure S1). If eligibility for inclusion was uncertain, we discussed the article with a third author (EVD) and included the paper by consensus of all three authors.

\section{Quality criteria}

Previous literature has indicated that network analyses may be subject to various methodological choices, for example the use of adequate connectivity measures ${ }^{18-21}$ and the definition of nodes and edges ${ }^{22-26}$ These methodological choices can introduce bias and strongly influence the outcomes of graph analysis $19,20,27,28$. Therefore, we developed a priori quality criteria based on state-of-the-art methodological studies ${ }^{19,20,29-31}$ Consensus papers from experts in the field of interest ${ }^{19,20,29}$ were used to assess the quality of the studies and quantify their impact (Figure 1, Supplementary Information Text Section A1). Based on these, two authors (CK and LM) evaluated each study independently and categorized the quality as excellent, good or moderate. If the scores differed between authors, a third author (EVD or SVM) evaluated the study, and the quality score was determined after consensus of all three authors. 


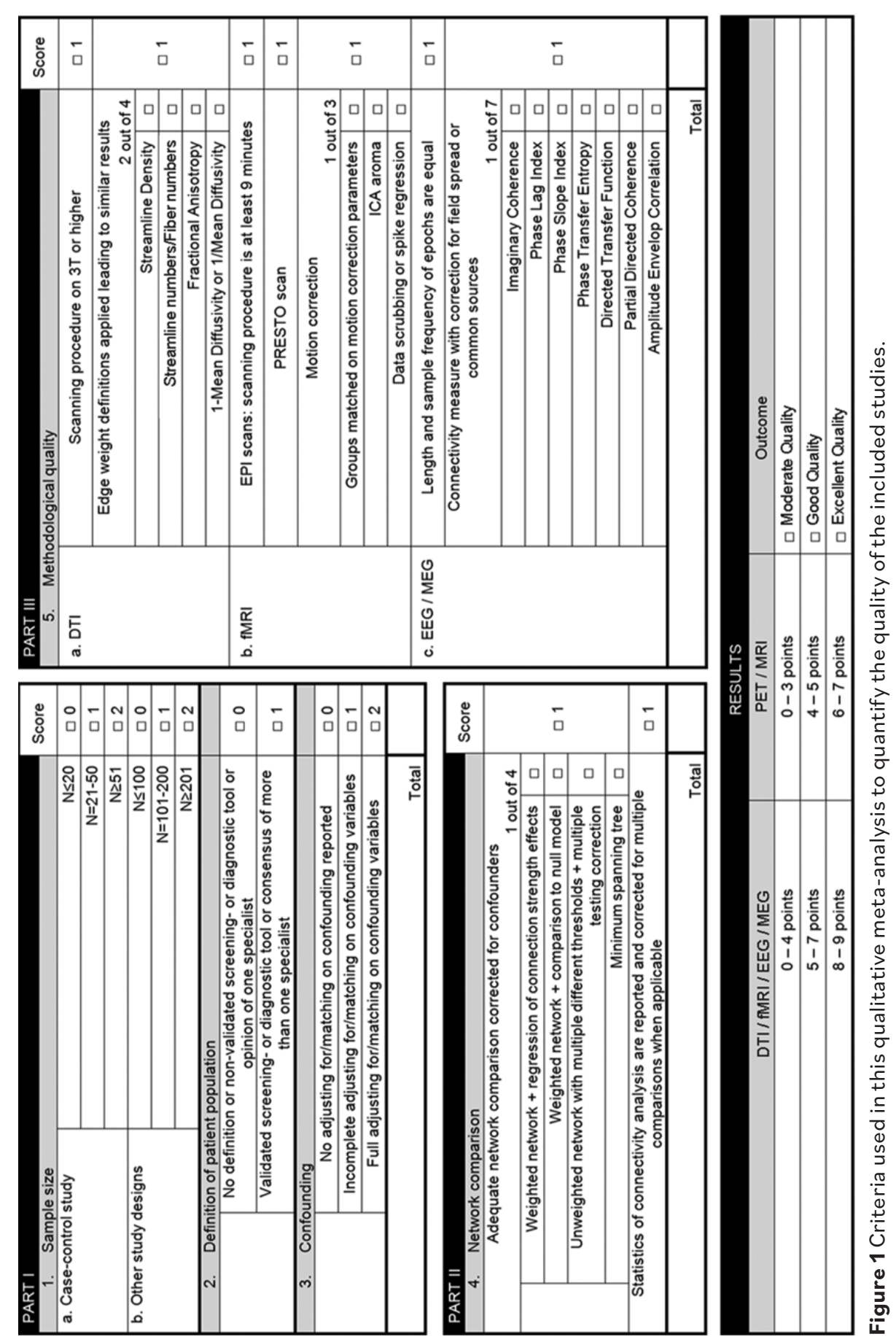

\section{Qualitative meta-analysis}

\section{Case-specific results}

Structural and functional brain network studies were separately analyzed for the different risk factors. As different imaging modalities measure various aspects of the structural and functional networks which should be interpreted differently, studies were grouped according to the imaging modality, i.e. white matter networks based on DTI, grey matter networks based on T1 structural MRI, functional networks based on fMRI and functional networks based on EEG or MEG. FMRI and EEG or MEG can be considered to give complementary information about functional interactions between brain areas, where the spatial resolution of $\mathrm{fMR}$ provides more accurate anatomical information, whereas EEG and MEC provide a higher temporal resolution of functional connectivity. All good and excellent quality studies for each modality (DTI, MRI grey matter networks, fMRI/PET, EEG/MEG) were compared per risk factor and outcome measure (connectivity strength, global efficiency, local clustering and modularity). Results of the outcomes were selected from the articles by two authors (AA and SVM) independently and checked by two other authors independently (LM and CK). If comparison of the outcomes extracted by both authors produced contradictory results, the authors discussed this with a third author (EVD), and adapted the outcome after consensus of all three authors. If a publication explored more than one risk factor separately, we took the comparison of each risk factor as a separate result, referred to as case.

Composite scores

As methods used to perform graph analyses were not equal between the different included studies, a quantitative meta-analysis appeared not to be feasible. However, to study whether delirium and its risk factors are associated with consistent brain network changes we performed a qualitative meta-analysis, in which we summarized results of the different included studies in composite scores. A composite score for each modality (DTI, MRI grey matter networks, fMRI/PET, EEG/MEG) and each outcome measure (connectivity strength, global efficiency, local clustering and modularity) was calculated. After exclusion of the moderate quality studies, all studies were given an equal weight in the composite score of the risk factor. The result of the composite score was one of the following: (a) "no 
effect", i.e. outcome was assessed, but the majority of studies found no effect of the risk factor on this outcome, (b) "higher" outcome value, i.e. the majority of investigations found an increase of this outcome measure associated with the risk factor, (c) "lower" outcome value, i.e. the majority of studies found a decrease of this outcome measure associated with the risk, (d) "inconclusive" outcome value, the more than $50 \%$ of the investigations reported contradictory results, (e) "not measured", i.e. no studies assessing this outcome were available for this risk factor. The composite score was accompanied with the percentage of studies representing the score (i.e. "no effect", "higher", "lower"). For example, if 5 DTI studies on the risk factor aging assessed the outcome global efficiency, of which 4 studies found a decreased global efficiency in older subjects, the composite score was "lower: 4 out of 5". Outcomes of moderate studies were qualitatively described in the results section if no good or excellent quality studies were available.

\section{Results}

Our literature search resulted in 24442 hits of which 126 studies met our inclusion criteria (Supplementary Information Figure S2). These 126 publications described in total 151 cases on different predisposing risk factors, precipitating risk factors or delirium (i.e. if a publication explored more than one risk factor separately, we took the comparison of each risk factor as a separate case) ${ }^{10,32-156}$. For a detailed overview of included studies, investigated risk factors, measurement techniques, outcomes and quality scores see Supplementary Information Table S3. After scoring, 118 cases were graded as qualitatively 'good or excellent', of which 99 on predisposing risk factors, 11 on precipitation risk factors and 7 on delirium, and included in our risk factor composite scores. Table 1 show findings for each modality: structural networks based on MRI grey matter similarity, structural networks based on DTI, functional networks based on $\mathrm{fMRI}$, and functional networks based on EEG. Below we describe findings on risk factors with at least 2 good or excellent quality studies, if not otherwise specified.

\section{Predisposing delirium risk factors and structural networks}

White matter networks

DTI-based structural network studies generally showed an association of predisposing risk factors for delirium with lower connectivity strength and lower network efficiency (Table 1, part 1A). Aging (2 out of $2(2 / 2)$ studies), cognitive impairment (2/2 studies) and depression (2/3 studies) were associated with lower connectivity strength $35,57,60,111,118,122$. Aging (2/2 studies), cognitive impairment (5/7 studies), dementia ( $3 / 4$ studies) and visual impairment ( 1 study) were all associated with lower network efficiency 35,57-63,94-96,111,154. Depression (6/7 studies) showed however no effect on efficiency and stroke $(\mathrm{N}=2)$ showed contradictory findings on efficiency $57,62,116-120,122,146$. The majority of risk factors showed no effect on local clustering 35,57-62,94-96,110,111,116,118-120,122,146,154. Mixed results were found for different risk factors for modularity: while one study on aging showed no effect ${ }^{111}$, a study on dementia showed increased modularity ${ }^{94}$.

\section{Grey matter networks}

Evidence for grey matter network alterations due to delirium predisposing risk factors was scarce (Table 1, part 1B). The two studies on aging both showed an association between aging and loss of efficiency ${ }^{32,34}$. However, no effect on grey matter network efficiency was found for cognitive impairment $(\mathrm{N}=5)$, dementia $(\mathrm{N}=6)$ and depression $(\mathrm{N}=6)$ in at least $50 \%$ of studies 51-53,55,90-93,111-115,117. Inconsistent results were found for various delirium risk factors on strength, local clustering and modularity ${ }^{32-34,45,51-}$ $55,90,92,93,111-115,117,137$

\section{Predisposing delirium risk factors and functional networks} fMRI and PET

fMRI-based functional network studies generally showed an association of predisposing risk factors for delirium and lower connectivity strength (Table 1, part 1C). Aging (2/3 studies) and dementia (2/3 studies) were associated with lower fMRI connectivity strength $38-40,102,103$. The same effect was found for cognitive impairment (2/2 studies) ${ }^{65,66}$, but these studies were of moderate quality. Regarding efficiency, most of the risk factors reported conflicting results on $\mathrm{fMRI}$ and PET networks (cognitive impairment: $\mathrm{N}=10$, dementia: $\mathrm{N}=11$ and hearing loss: $\mathrm{N}=2)^{71-73,75-77,98,99,102,103,138,139}$. Aging (3/4 
studies) and depression (4/7 studies) were associated with no effect on efficiency in $\mathrm{fMRI}$ and PET studies 38,39,41,45,64,67-69,71-73,75-77,124-126,128-130,157. For local clustering, $\mathrm{fMRI}$ and PET studies on dementia $(\mathrm{N}=11)$ and hearing impairment ( $N=2)$ showed conflicting results as well $71-73,76,77,98,99,102,103,138,139$ while most $\mathrm{fMRI}$ and PET studies on cognitive impairment (6/9 studies) and depression (5/6 studies) showed no effect ${ }^{69,71-73,76,77,124-126,128,157}$. Although al studies on aging (4/4 studies) showed decreased modularity ${ }^{38,39,41,42}$, studies on other risk factors showed inconclusive findings (cognitive impairment: $\mathrm{N}=6$ ) or no effect on modularity (4/5 studies) 64,68,69,71-73,102.

\section{$E E G$ and $M E G$}

EEG and MEG-based functional network studies showed mixed results with a tendency towards lower connectivity strength in the alpha band (Table 1 , part 1D). Specifically, aging ( $2 / 2$ studies) and cognitive impairment ( $2 / 3$ studies) were associated with a decreased EEG connectivity strength in the alpha band $49,50,78,79,81$. Studies on alpha band connectivity strength in dementia showed mixed results of decreased connectivity strength (1/2 studies) and no effect ( $1 / 2$ studies). However, two moderate quality studies also showed decreased alpha band connectivity strength ${ }^{80,108}$. No effects were found for other frequency bands. Mixed results for different risk factors were found on efficiency, local clustering and modularity $46,47,50,78$ ,79,81,106,107,132,133,147.

\section{Precipitating delirium risk factors and functional networks}

\section{fMRI}

Evidence for $\mathrm{fMRI}$ network alterations due to delirium-precipitating risk factors was scarce (Table 1, part 2A). Sedation ( $2 / 3$ studies) and renal failure $(N=1)$ were associated with decreased efficiency ${ }^{141-143}$, but coma $(\mathrm{N}=2)$ and neurotrauma $(\mathrm{N}=1)$ showed no effect on efficiency ${ }^{84,85,150}$. Mixed results for different risk factors were found for strength, local clustering and modularity $84,85,142,143,150,151$

\section{$E E G$ and $M E C$}

EEG and MEG-based functional network studies generally showed an association of precipitating risk factors for delirium with lower efficiency and a higher local clustering in the alpha band (Table 1, part 2B). Sedation (2/3 studies) and neurotrauma ( $\mathrm{N}=1$ of moderate quality) were associated with a decreased efficiency in the alpha band ${ }^{89,144,145,153}$. Sedation (2/2 studies) and neurotrauma ( $\mathrm{N}=1$ of moderate quality) were further associated with increased local clustering in the alpha band ${ }^{144,145,153}$. No effect was found in these two risk factors on connectivity strength $89,144,145,153$

\section{Delirium and functional networks}

fMRI

Evidence for $\mathrm{fMRI}$ network alterations in delirium was scarce (Table 1, part 3A). Only one fMRI study during delirium was detected, showing a loss in efficiency and local clustering ${ }^{86}$. Modularity was not assessed in this study. Three fMRI studies on hepatic encephalopathy ${ }^{87,134,135}$ (of which one of moderate quality ${ }^{135}$ ) did not show loss of efficiency, and reported decreased local clustering ${ }^{87,134,135}$. Two fMRI studies on hepatic encephalopathy (of which one of moderate quality ${ }^{135}$ showed decreased modularity ${ }^{136}$.

$E E G$

EEG-based functional network studies showed an association of delirium with lower connectivity strength in the alpha band (Table 1, part 3B). A decreased connectivity strength in the alpha band was reported in the available EEG publications ( $3 / 3$ studies) ${ }^{10,88,89}$, but two of these were based on the same dataset. No effect on local clustering ${ }^{10,89}$ was found ( $2 / 2$ studies). An inconclusive effect on alpha band efficiency was found due to methodological differences between studies ${ }^{9,86}$. Using the minimum spanning tree (MST) diameter, a less biased measure of efficiency than the path length of a weighted network ${ }^{158,159}$, a decreased alpha band efficiency was observed. 

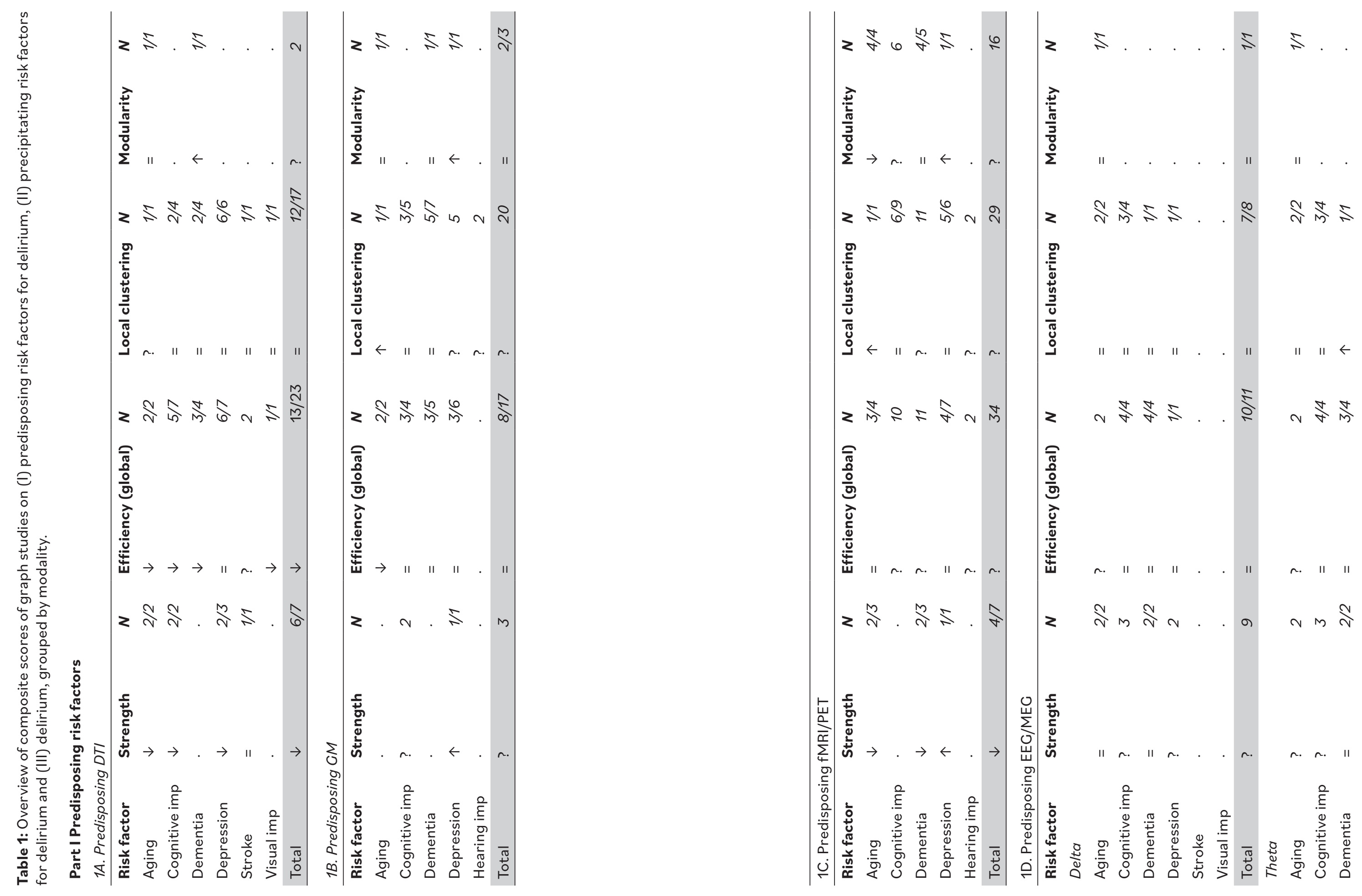

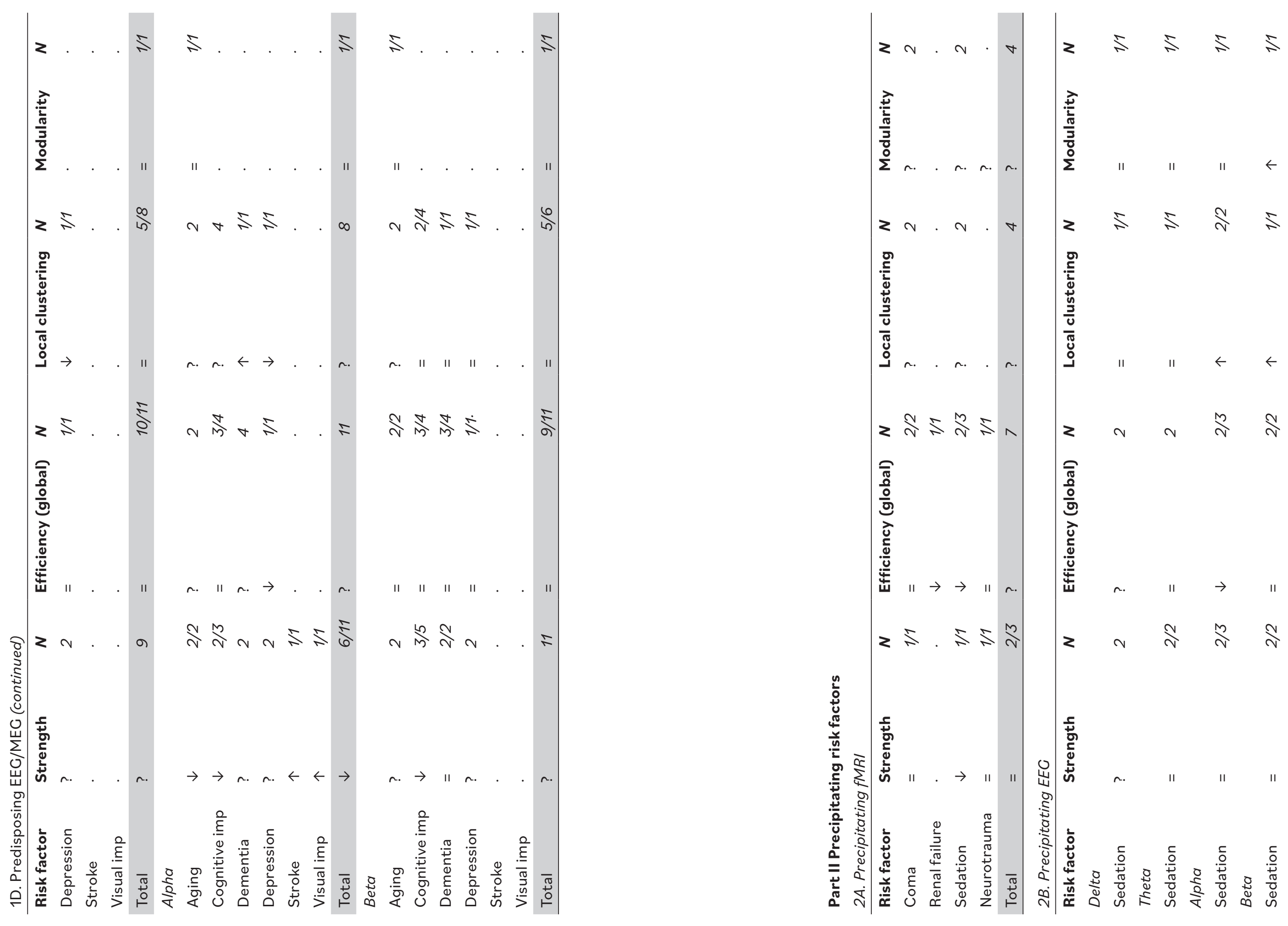


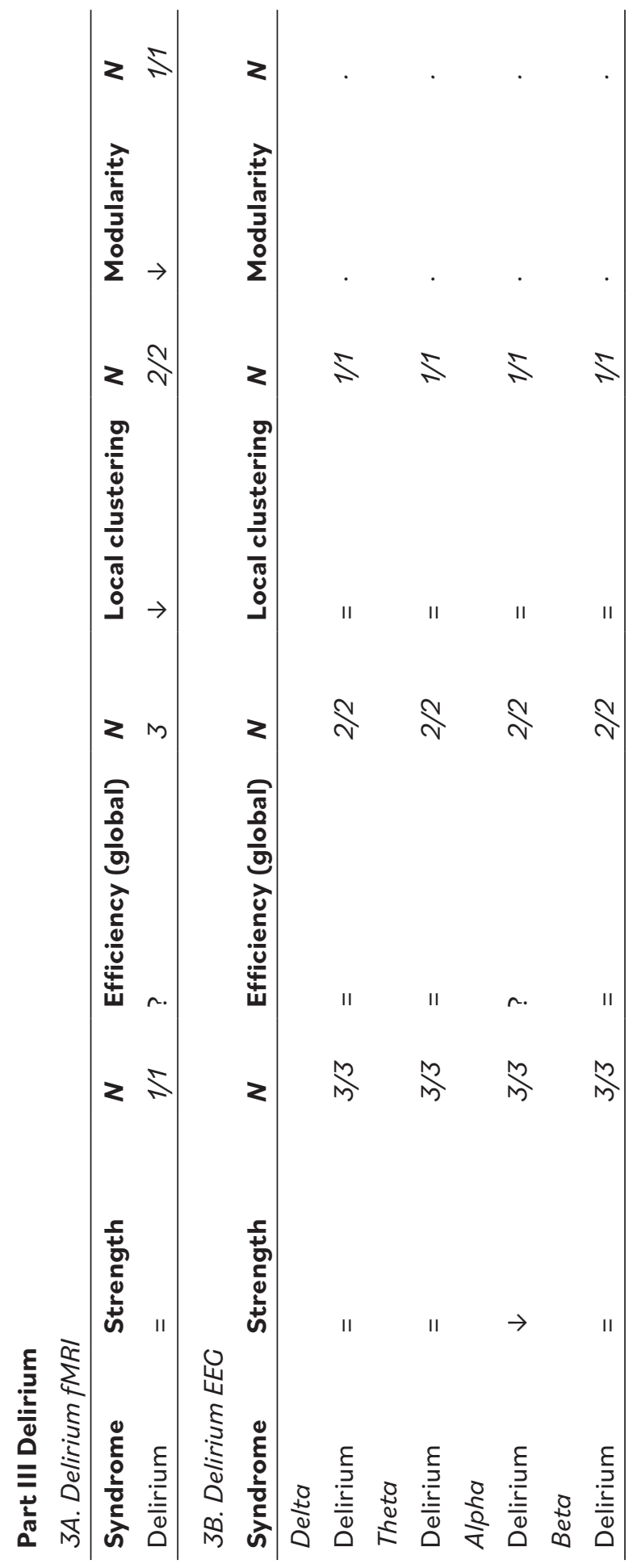

\section{Discussion}

We evaluated the evidence for alterations in the structural and functional brain network related to delirium and its risk factors (Figure 2). On a structural level, predisposing risk factors were generally associated with lower connectivity strength and less efficient organization of white matter connections. On a functional level, a decrease of functional connectivity strength was found in most fMRI- and some EEG studies related to predisposing risk factors. The limited $\mathrm{FMRI}$ and EEG data available on precipitating factors generally indicated less efficiency of functional networks. During delirium, functional brain networks were characterized by decreased alpha band EEG connectivity and lower fMRI network integration. Taken together, we found evidence that a less connected and less integrated brain network is a common mechanism in the pathophysiology of delirium.

\section{Effects of predisposing delirium risk factors on brain networks}

Although all studied risk factors were generally associated with decreased strength and loss of efficiency, most conclusive evidence for brain network alterations was found for aging, dementia and cognitive impairment. However, depression showed an aberrant effect in global efficiency of structural networks. A possible explanation is that depression is a more heterogeneous disorder with a largely unknown biological substrate ${ }^{160}$, making it difficult to compare studies within this risk factor. The risk factor age showed a stronger risk factor-specific pattern compared to other risk factors. Investigations on aging showed decreased efficiency in grey matter MRI studies and loss of modularity in $\mathrm{fMRI}$ studies, while findings on other risk factors were inconclusive or absent. Aging is known as a key risk factor for delirium ${ }^{12,31}$, which may be related to its extensive impact on brain network topology. 


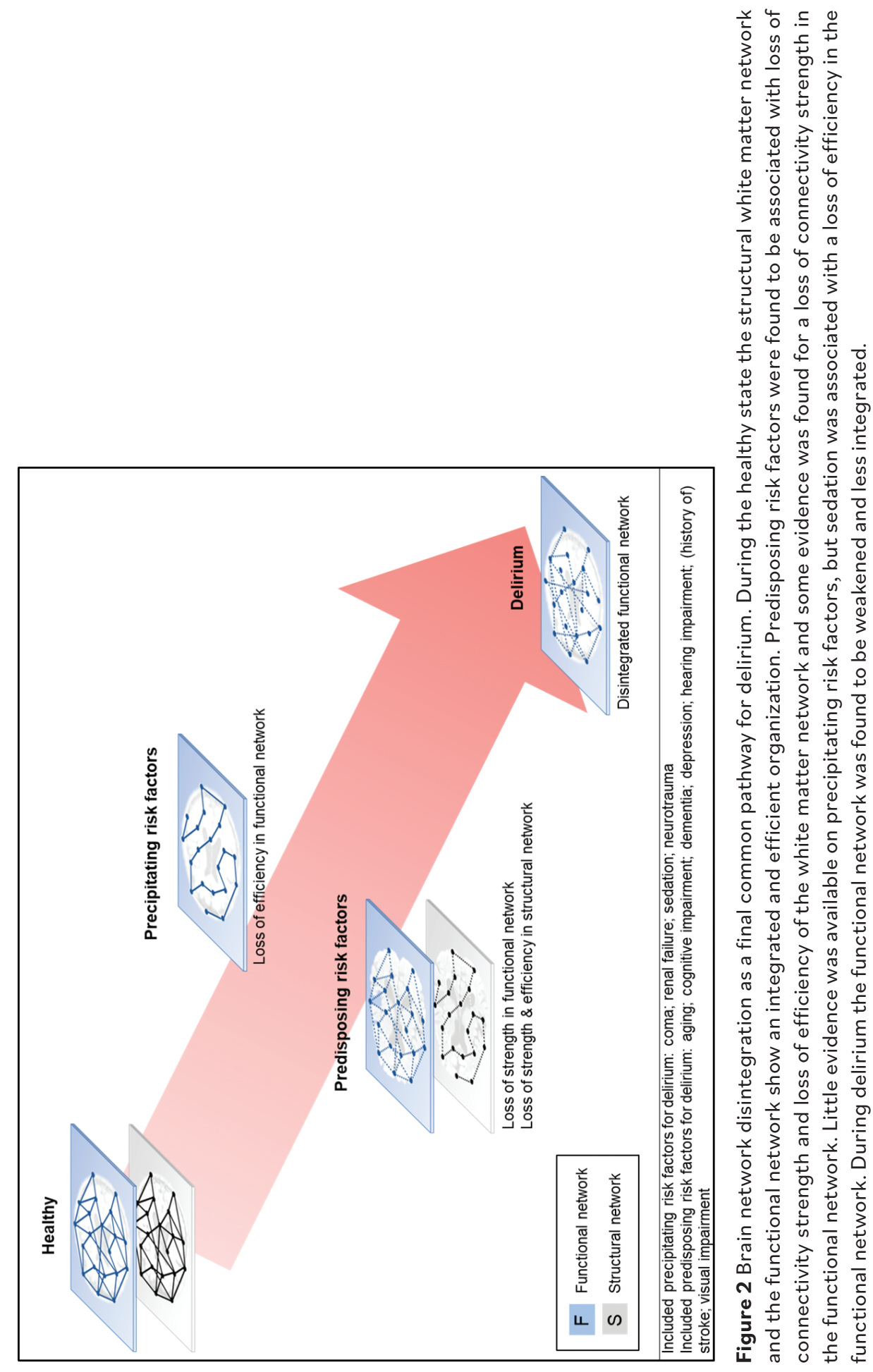

\section{Effects of delirium on brain networks}

During delirium, a variety of network changes have been observed, i.e. reduced connectivity strength, reduced global efficiency, reduced local clustering and reduced modularity, although the number of investigations was small. In general the strongest evidence was found for a less connected and disintegrated network during the syndrome. Due to the limited amount of studies, we are currently unable to distinguish specific network alterations to the different clinical subtypes of delirium.

\section{Strengths and limitations}

The framework of graph theory provides new opportunities to study the development of neuropsychiatric diseases. Our rigorous systematic review and qualitative meta-analysis revealed new insights on the pathophysiology of delirium. The development and use of the quality criteria for network studies, largely based on recent consensus papers on methodological approaches, allowed us to assess the robustness of findings ${ }^{19,20,27,28}$. These quality criteria can be used and adapted for future investigations on other topics.

We studied a variety of presumed delirium risk factors in relation to brain network alterations. As there is no general consensus which factors increase the risk of delirium, it could be argued that inclusion of some of these factors may have biased our analyses. In the absence of strong epidemiological evidence on the exact risk profile of delirium, we included delirium risk factors based on a recent landmark article published in a high-impact medical journal ${ }^{12}$

Comparing brain network outcomes of different studies in a qualitative way may be unconventional. The outcomes of the studies were similar, but some studies differed in study design and exact calculations of the outcomes. Moreover, efficiency estimates may be biased by connection strength ${ }^{25,26,28}$, which may be relevant for our qualitative analysis. A qualitative assessment suggested that efficiency loss due to delirium risk factors may at least partially be explained by lower connectivity strength, but average connectivity was not reported as outcome measure in the majority of cases (results in Supplementary Information Text Section A2). 
Future work, implementing recently introduced corrections for this possible confounder ${ }^{26,159}$, is needed to show if efficiency loss is present independent of connectivity strength effects. Observations of decreased connectivity strength and loss of network efficiency have been associated to other disorders as well, and may therefore not be specific for the pathophysiology of delirium ${ }^{2,3,162}$.

As positive and negative results are not equally reported in the literature 163,164 , our review may have been influenced by publication bias. We have attempted to reduce this bias by defining the risk factors for delirium on a previously published landmark paper ${ }^{12}$, by using a predefined systematic search term and by conducting our search in two different libraries, i.e. PubMed and EMBASE. However, like in other systematic reviews and metaanalyses, unpublished negative studies could not be included.

Delirious patients can be restless or agitated ${ }^{6}$, which may have influenced the quality of EEG and $\mathrm{FMRI}$ measurements ${ }^{22,29}$. Although in EEG analyses artifact-free epochs were used and usage of $\mathrm{fMRI}$ motion correction was part of our quality criteria, the results shown in this study may still have been (partly) effected by motion. Future studies on delirium may benefit from strict motion correction. In addition, patients with delirium always suffer from an underlying physical condition and may use a variety of medication, which may have influenced the brain status. However, the included studies on delirium all used a clinically matched control group to minimize medication effects (and other hospitalization), and in some studies patients were even matched on (specific types of) medication use. Furthermore, antipsychotics such as haloperidol may not particularly influence measures of brain function ${ }^{165}$. Likewise, delirious patients could suffer from brain damage, which might have led to differences in brain function ${ }^{2,166}$. This may however not be the essential factor for network disruptions during delirium as studies that strictly corrected for brain lesions in their study sample report similar results as studies that did not ${ }^{86,88}$.

Neuropsychiatric disorders may be associated with alterations of hubs in the network ${ }^{2,3}$. Hubs were not considered in the current study because of the lack of a formal definition of hubs, together with the small number of studies using hubs as a comparable outcome measure. Not all factors influencing vulnerability for delirium have been studied in relation to brain network alterations. Future work is needed to validate our hypothesis for other delirium risk factors and to integrate the framework of graph theory and brain networks with other biological processes underlying delirium.

\section{A network model of delirium}

Our findings suggest that delirium predisposition is associated with a less connected and less efficient structural network, and a less connected functional network. Structural and functional network organization are closely related ${ }^{167}$, and this relation may be of particular relevance for the pathophysiology of delirium. Computational studies have shown that reduced structural connectivity strength as characterized by reduced white matter volume, can cause decreased functional connectivity strength and efficiency ${ }^{168,169}$. Moreover, weakening of structural network efficiency may decrease global spreading of information in the functional networks, disabling cooperative effects between network components 170. Precipitating delirium risk factors may cause further loss of functional brain network efficiency towards a critical transition ${ }^{167,168}$, consequently inducing an acute global loss of functional interactions and network integration, as seen in functional connectivity studies in delirium patients $10,86,89$. Accordingly, white matter network studies on delirious patients or patients at risk for delirium, specifically show disturbances in white matter network strength and efficiency ${ }^{171,172}$, strengthening the evidence for our proposed network model of delirium.

The theory of alterations of brain networks do not have to replace other hypotheses on the pathophysiology of delirium. Important theories on the etiology of delirium include persistent neuroinflammation, an aberrant stress response and alterations of neurotransmission ${ }^{173}$. It remains to be studied to what extent these are associated with brain network alterations. A recent modeling study showed that EEG phenomena associated with delirium, including connectivity and network alterations, may be the result of imbalance between excitatory and inhibitory activity, as well as increased fluctuations in subcortical information ${ }^{174}$. Particularly an altered balance between glutamatergic and GABAergic neurotransmission may contribute 
to network vulnerability ${ }^{9}$. Previous studies have shown GABAergic medication, including benzodiazepines, as precipitant of delirium ${ }^{31}$ and reduced network connectivity ${ }^{175}$.

At present, management of delirium consists of symptomatic treatment and treatment of underlying conditions, while there is no proven intervention that directly improves the underlying brain dysfunction. There is therefore a need for targeted interventions focused on the pathophysiology of the disorder. Non-invasive targeted brain stimulation, such as transcranial direct current stimulation (tDSC) and Transcranial Magnetic Stimulation (TMS), may normalize the functional brain network and can have beneficial therapeutic effects in several groups of (neuro)psychiatric patients ${ }^{176,177}$ Based on the proposed model for delirium, we suggest that these networkbased interventions, may be promising for delirium treatment.

\section{Conclusion}

Decreased connectivity strength and efficiency seem to characterize structural brain networks of patients at risk for delirium, while functional network disintegration appears to be the final common pathway for the syndrome.

\section{Acknowledgements}

This research did not receive any specific grant from funding agencies in the public, commercial, or not-for-profit sectors. We would like to especially thank Luna Wattel for her great support in the layout of the figures.

\section{References}

1. Sporns 0 . Contributions and challenges for network models in cognitive neuroscience. Nat Neurosci. 2014;17(5):652-660. doi:10.1038/nn.3690

2. Stam CJ. Modern network science of neurological disorders. Nat Rev Neurosci. 2014;15(10):683-695. doi:10.1038/nrn3801

3. Crossley NA, Mechelli A, Scott J, et al. The hubs of the human connectome are generally implicated in the anatomy of brain disorders. Brain. 2014;137(8):2382-2395 doi:10.1093/brain/awu132

4. Douw L, van Dellen E, de Groot M, et al. Epilepsy is related to theta band brain connectivity and network topology in brain tumor patients. BMC Neurosci. 2010;11(1):103. doi:10.1186/1471-2202-11-103

5. van Diessen E, Diederen SJH, Braun KPJ, Jansen FE, Stam CJ. Functional and structural brain networks in epilepsy: What have we learned? Epilepsia. 2013;54(11):1855-1865. doi:10.1111/epi.12350

6. American Psychiatric Association. Diagnostic and Statistical Manual of Mental Disorders. American Psychiatric Association; 2013. doi:10.1176/appi.books.9780890425596

7. Yang FM, Marcantonio ER, Inouye SK, et al. Phenomenological Subtypes of Delirium in Older Persons: Patterns, Prevalence, and Prognosis. Psychosomatics. 2009;50(3):248254. doi:10.1176/APPI.PSY.50.3.248

8. Marcantonio ER. Delirium in Hospitalized Older Adults. Solomon CG, ed. N EnglJ Med. 2017;377(15):1456-1466. doi:10.1056/NEJMcp1605501

9. Sanders RD. Hypothesis for the pathophysiology of delirium: Role of baseline brain network connectivity and changes in inhibitory tone. Med Hypotheses. 2011;77(1):140 143. doi:10.1016/j.mehy.2011.03.048

10. van Dellen E, van der Kooi AW, Numan T, et al. Decreased Functional Connectivity and Disturbed Directionality of Information Flow in the Electroencephalography of Intensive Care Unit Patients with Delirium after Cardiac Surgery. Anesthesiology. 2014;121(2):328-335. doi:10.1097/ALN.0000000000000329

11. Young JWS. The network model of delirium. Med Hypotheses. 2017;104:80-85 doi:10.1016/j.mehy.2017.05.027

12. Inouye SK, Westendorp RG, Saczynski JS. Delirium in elderly people. Lancet 2014;383(9920):911-922. doi:10.1016/S0140-6736(13)60688-1

13. Stam CJ, Reijneveld JC. Graph theoretical analysis of complex networks in the brain Nonlinear Biomed Phys. 2007;1(1):3. doi:10.1186/1753-4631-1-3

14. Biggs N, Lloyd EK, Wilson RJ. Graph Theory, 1736-1936. Oxford: Clarendon Press; 1986 doi:10.1086/352170 
15. Williams ST. Pathophysiology of Encephalopathy and Delirium. J Clin Neurophysiol 2013;30(5):435-437. doi:10.1097/WNP.0b013e3182a73e04

16. Brown E, Douglas V. Moving Beyond Metabolic Encephalopathy: An Update on Delirium Prevention, Workup, and Management. Semin Neurol. 2015;35(6):646-655. doi:10.1055/s-0035-1564685

17. Maldonado JR. Acute Brain Failure: Pathophysiology, Diagnosis, Management, and Sequelae of Delirium. Crit Care Clin. 2017;33(3):461-519. doi:10.1016/j.ccc.2017.03.013

18. Chunmei Zhang, Wenxue Li, Ke Wang. Graph Theory-Based Approach for Stability Analysis of Stochastic Coupled Systems With Levy Noise on Networks. IEEE Trans Neural Networks Learn Syst. 2015;26(8):1698-1709. doi:10.1109/TNNLS.2014.2352217

19. van Diessen E, Numan $T$, van Dellen E, et al. Opportunities and methodological challenges in EEG and MEG resting state functional brain network research. Clin Neurophysiol. 2015;126(8):1468-1481. doi:10.1016/j.clinph.2014.11.018

20. Fornito A, Zalesky A, Breakspear M. Graph analysis of the human connectome: Promise, progress, and pitfalls. Neuroimage. 2013;80:426-444. doi:10.1016/j. neuroimage.2013.04.087

21. Rubinov M, Sporns O. Complex network measures of brain connectivity Uses and interpretations. Neuroimage. 2010;52(3):1059-1069. doi:10.1016/j. neuroimage.2009.10.003

22. Power JD, Schlaggar BL, Petersen SE. Recent progress and outstanding issued in motion correction resting state fmri. Neuroimage. 2015;105:536-551. doi:10.1016/j. neuroimage.2014.10.044.Recent

23. Pruim RHR, Mennes M, Buitelaar JK, Beckmann CF. Evaluation of ICA-AROMA and alternative strategies for motion artifact removal in resting state fMRI. Neuroimage. 2015;112:278-287. doi:10.1016/j.neuroimage.2015.02.063

24. Dijk KRA Van, Sabuncu MR, Buckner RL. Neurolmage The in fl uence of head motion on intrinsic functional connectivity MRI. Neuroimage. 2012;59(1):431-438. doi:10.1016/j. neuroimage.2011.07.044

25. van Wijk BCM, Stam CJ, Daffertshofer A, Stam C, Apkarian A. Comparing Brain Networks of Different Size and Connectivity Density Using Graph Theory. Sporns O, ed. PLoS One. 2010;5(10):e13701. doi:10.1371/journal.pone.0013701

26. van den Heuvel MP, de Lange SC, Zalesky A, Seguin C, Yeo BTT, Schmidt R. Proportional thresholding in resting-state $\mathrm{fMRI}$ functional connectivity networks and consequences for patient-control connectome studies: Issues and recommendations. Neuroimage. 2017;152:437-449. doi:10.1016/J.NEUROIMAGE.2017.02.005
27. Alderson-Day B, Diederen K, Fernyhough C, et al. Auditory Hallucinations and the Brain's Resting-State Networks: Findings and Methodological Observations. Schizophr Bull. 2016;42(5):1110-1123. doi:10.1093/schbul/sbw078

28. Tijms BM, Wink AM, de Haan W, et al. Alzheimer's disease: connecting findings from graph theoretical studies of brain networks. Neurobio/ Aging. 2013;34(8):2023-2036. doi:10.1016/j.neurobiolaging.2013.02.020

29. Ciric R, Wolf DH, Power JD, et al. Benchmarking of participant-level confound regression strategies for the control of motion artifact in studies of functional connectivity. Neuroimage. 2017;154:174-187. doi:10.1016/j.neuroimage.2017.03.020

30. Birn, R.M., Molloy, E.K., Patriat, R., Parker, T., Meier, T.B., Kirk, G.R., Nair, V.A., Meyerand, M.E., Prabhakaran V. The effect of scan length on the reliability of restingstate fMRI connectivity estimates. Neuroimage. 2013;83:550-558. doi:10.1016/J. NEUROIMAGE.2013.05.099

31. Zaal IJ, Devlin JW, Peelen LM, Slooter AJC. A Systematic Review of Risk Factors for Delirium in the ICU*. Crit Care Med. 2015;43(1):40-47. doi:10.1097/ CCM.0000000000000625

32. Zhu W, Wen W, He Y, Xia A, Anstey KJ, Sachdev P. Changing topological patterns in normal aging using large-scale structural networks. Neurobiol Aging. 2012;33(5):899913. doi:10.1016/j.neurobiolaging.2010.06.022

33. Chen ZJ, He Y, Rosa-Neto P, Gong G, Evans AC. Age-related alterations in the modular organization of structural cortical network by using cortical thickness from MRI. Neuroimage. 2011;56(1):235-245. doi:10.1016/j.neuroimage.2011.01.010

34. Wu K, Taki Y, Sato K, Qi H, Kawashima R, Fukuda H. A longitudinal study of structural brain network changes with normal aging. Front Hum Neurosci. 2013;7:113. doi:10.3389/ fnhum.2013.00113

35. Otte WM, van Diessen E, Paul S, et al. Aging alterations in whole-brain networks during adulthood mapped with the minimum spanning tree indices: The interplay of density, connectivity cost and life-time trajectory. Neuroimage. 2015;109:171-189. doi:10.1016/J.NEUROIMAGE.2015.01.011

36. Lim S, Han CE, Uhlhaas PJ, Kaiser M. Preferential detachment during human brain development: age- and sex-specific structural connectivity in diffusion tensor imaging (DTI) data. Cereb Cortex. 2015;25(6):1477-1489. doi:10.1093/cercor/bht333

37. Gong G, Rosa-Neto P, Carbonell F, Chen ZJ, He Y, Evans AC. Age- and gender-related differences in the cortical anatomical network.J Neurosci. 2009;29(50):15684-15693. doi:10.1523/JNEUROSCI.2308-09.2009 
38. Geerligs L, Rubinov M, Cam-Can, Henson RN. State and Trait Components of Functional Connectivity: Individual Differences Vary with Mental State. J Neurosci. 2015;35(41):13949-13961. doi:10.1523/JNEUROSCI.1324-15.2015

39. SongJ, Birn RM, Boly M, et al. Age-Related Reorganizational Changes in Modularity and Functional Connectivity of Human Brain Networks. Brain Connect. 2014;4(9):662-676. doi:10.1089/brain.2014.0286

40. Ferreira LK, Regina ACB, Kovacevic N, et al. Aging effects on whole-brain functional connectivity in adults free of cognitive and psychiatric disorders. Cereb Cortex. 2016;26(9):3851-3865. doi:10.1093/cercor/bhv190

41. Cao M, Wang JH, Dai ZJ, et al. Topological organization of the human brain functional connectome across the lifespan. Dev Cogn Neurosci. 2014;7:76-93. doi:10.1016/j. dcn.2013.11.004

42. Chan MY, Park DC, Savalia NK, Petersen SE, Wig GS. Decreased segregation of brain systems across the healthy adult lifespan. Proc Natl Acad Sci. 2014;111(46):E4997-E5006. doi:10.1073/pnas.1415122111

43. Onoda K, Yamaguchi S. Small-worldness and modularity of the resting-state functiona brain network decrease with aging. Neurosci Lett. 2013;556:104-108. doi:10.1016/j. neulet.2013.10.023

44. Meunier D, Achard S, Morcom A, Bullmore E. Age-related changes in modular organization of human brain functional networks. Neuroimage. 2009;44(3):715-723. doi:10.1016/J.NEUROIMAGE.2008.09.062

45. Liu Z, Ke L, Liu H, Huang W, Hu Z. Changes in Topological Organization of Functional PET Brain Network with Normal Aging. Chen C-T, ed. PLoS One. 2014;9(2):e88690. doi:10.1371/journal.pone.0088690

46. Knyazev GG, Volf N V, Belousova L V. Age-related differences in EEG connectivity and network topology. Neurobiol Aging. 2015;36(5):1849-1859. doi:10.1016/j. neurobiolaging.2015.02.007

47. Vecchio F, Miraglia F, Marra C, et al. Human brain networks in cognitive decline: a graph theoretical analysis of cortical connectivity from EEG data. J Alzheimers Dis. 2014;41(1):113-127. doi:10.3233/jad-132087

48. Micheloyannis S, Vourkas M, Tsirka V, Karakonstantaki E, Kanatsouli K, Stam CJ. The influence of ageing on complex brain networks: A graph theoretical analysis. Hum Brain Mapp. 2009;30(1):200-208. doi:10.1002/hbm.20492

49. Vysata O, Kukal J, Prochazka A, Pazdera L, Simko J, Valis M. Age-related changes in EEC coherence. Neurol Neurochir Pol. 2014;48(1). doi:10.1016/j.pjnns.2013.09.001
50. Smit DJA, de Geus EJC, Boersma M, Boomsma DI, Stam CJ. Life-Span Development of Brain Network Integration Assessed with Phase Lag Index Connectivity and Minimum Spanning Tree Graphs. Brain Connect. 2016;6(4):312-325. doi:10.1089/ brain.2015.0359

51. Yao Z, Zhang Y, Lin L, Zhou Y, Xu C, Jiang T. Abnormal Cortical Networks in Mild Cognitive Impairment and Alzheimer's Disease. Friston KJ, ed. PLoS Comput Biol. 2010;6(11):e1001006. doi:10.1371/journal.pcbi.1001006

52. Phillips DJ, Mcglaughlin A, Ruth D, Jager LR, Soldan A. Neurolmage : Clinical Graph theoretic analysis of structural connectivity across the spectrum of Alzheimer 3 s disease: The importance of graph creation methods. YNICL. 2015;7:377-390. doi:10.1016/j.nicl.2015.01.007

53. Pereira JB, Aarsland D, Ginestet CE, et al. Aberrant cerebral network topology and mild cognitive impairment in early Parkinson's disease. Hum Brain Mapp. 2015;36(8):29802995. doi:10.1002/hbm.22822

54. Li Y, Wang Y, Wu G, et al. Discriminant analysis of longitudinal cortical thickness changes in Alzheimer's disease using dynamic and network features. Neurobiol Aging. 2012;33(2):427.e15-30. doi:10.1016/j.neurobiolaging.2010.11.008

55. Li Q, Li X, Wang X, et al. Topological properties of large-scale cortical networks based on multiple morphological features in amnestic mild cognitive impairment. Neural Plast. 2016;2016:3462309. doi:10.1155/2016/3462309

56. Wang $X-N$, Zeng $\mathrm{Y}$, Chen $\mathrm{G}-\mathrm{Q}$, et al. Abnormal organization of white matter networks in patients with subjective cognitive decline and mild cognitive impairment. Oncotarget. 2016;7(31):48953-48962. doi:10.18632/oncotarget.10601

57. Bai F, Shu N, Yuan Y, et al. Topologically Convergent and Divergent Structural Connectivity Patterns between Patients with Remitted Geriatric Depression and Amnestic Mild Cognitive Impairment.J Neurosci. 2012;32(12):4307-4318. doi:10.1523/ JNEUROSCI.5061-11.2012

58. Daianu M, Jahanshad N, Nir TM, et al. Breakdown of Brain Connectivity Between Normal Aging and Alzheimer's Disease: A Structural k -Core Network Analysis. Brain Connect. 2013;3(4):407-422. doi:10.1089/brain.2012.0137

59. Morris RG, Lawrence AJ, Chung AW, Barrick TR, Markus HS. Structural network efficiency is associated with cognitive impairment in small-vessel disease. Neurology. 2014;83(4):304-311. doi:10.1212/wnl.0000000000000612

60. Shu N, Liang Y, Li H, et al. Disrupted Topological Organization in White Matter Structural Networks in Amnestic Mild Cognitive Impairment: Relationship to Subtype. Radiology. 2012;265(2):518-527. doi:10.1148/radiol.12112361 
61. Vaessen M, Jansen J, Vlooswijk MCG, et al. White matter network abnormalities are associated with cognitive decline in chronic epilepsy. Cereb Cortex. 2012;22(9) doi:10.1093/cercor/bhr298

62. Tang J, Zhong S, Chen Y, et al. Aberrant White Matter Networks Mediate Cognitive Impairment in Patients with Silent Lacunar Infarcts in Basal Ganglia Territory.J Cereb Blood Flow Metab. 2015;35(9):1426-1434. doi:10.1038/jcbfm.2015.67

63. Zhao T, Sheng C, Bi Q, Niu W, Shu N, Han Y. Age-related differences in the topological efficiency of the brain structural connectome in amnestic mild cognitive impairment. Neurobio/ Aging. 2017;59:144-155. doi:10.1016/j.neurobiolaging.2017.08.005

64. Yi L-Y, Liang X, Liu D-M, et al. Disrupted Topological Organization of Resting-State Functional Brain Network in Subcortical Vascular Mild Cognitive Impairment. CNS Neurosci Ther. 2015;21(10):846-854. doi:10.1111/cns.12424

65. Minati L, Chan D, Mastropasqua C, et al. Widespread alterations in functional brain network architecture in amnestic mild cognitive impairment. J Alzheimers Dis. 2014;40(1):213-220. doi:10.3233/jad-131766

66. Chang T-Y, Huang K-L, Ho M-Y, et al. Graph theoretical analysis of functional networks and its relationship to cognitive decline in patients with carotid stenosis.J Cereb Blood Flow Metab. 2016;36(4):808-818. doi:10.1177/0271678X15608390

67. Yu Y, Zhou X, Wang H, et al. Small-World Brain Network and Dynamic Functional Distribution in Patients with Subcortical Vascular Cognitive Impairment. Walter M, ed. PLoS One. 2015;10(7):e0131893. doi:10.1371/journal.pone.0131893

68. Baggio H-C, Sala-Llonch R, Segura B, et al. Functional brain networks and cognitive deficits in Parkinson's disease. Hum Brain Mapp. 2014;35(9):4620-4634. doi:10.1002/ hbm.22499

69. Wang J, Zuo X, Dai Z, et al. Disrupted functional brain connectome in individuals at risk for Alzheimer's disease. Biol Psychiatry. 2013;73(5):472-481. doi:10.1016/j. biopsych.2012.03.026

70. Xiang J, Guo H, Cao R, Liang $\mathrm{H}$, Chen JJ. An abnormal resting-state functional brain network indicates progression towards Alzheimer's disease. Neural Regen Res. 2013;8(30):2789-2799. doi:http://dx.doi.org/10.3969/j.issn.1673-5374.2013.30.001

71. Brier MR, Thomas JB, Fagan AM, et al. Functional connectivity and graph theory in preclinical Alzheimer's disease. Neurobiol Aging. 2013;35(4):757-768. doi:S01974580(13)00549-6 [pii]\r10.1016/j.neurobiolaging.2013.10.081

72. Kim HK, Yoo K, Na DL, Seo SW, Jeong J, Jeong Y. Non-monotonic reorganization of brain networks with Alzheimer's disease progression. Front Aging Neurosci. 2015;7:111. doi:10.3389/fnagi.2015.00111
73. Sun Y, Yin Q, Fang R, et al. Disrupted Functional Brain Connectivity and Its Association to Structural Connectivity in Amnestic Mild Cognitive Impairment and Alzheimer's Disease. Tian J, ed. PLoS One. 2014;9(5):e96505. doi:10.1371/journal.pone.0096505

74. Liu Z, Zhang Y, Yan H, et al. Altered topological patterns of brain networks in mild cognitive impairment and Alzheimer's disease: a resting-state fMRI study. Psychiatry Res. 2012;202(2):118-125. doi:10.1016/j.pscychresns.2012.03.002

75. Sang L, Chen L, Wang L, et al. Progressively Disrupted Brain Functional Connectivity Network in Subcortical Ischemic Vascular Cognitive Impairment Patients. Front Neurol. 2018;9. doi:10.3389/fneur.2018.00094

76. Sanabria-Diaz G, Martínez-Montes E, Melie-Garcia L. Glucose Metabolism during Resting State Reveals Abnormal Brain Networks Organization in the Alzheimer's Disease and Mild Cognitive Impairment. Lin C-P, ed. PLoS One. 2013;8(7):e68860. doi:10.1371/journal.pone.0068860

77. Seo EH, Lee DY, Lee J-M, et al. Whole-brain Functional Networks in Cognitively Normal, Mild Cognitive Impairment, and Alzheimer's Disease. He Y, ed. PLoS One. 2013;8(1):e53922. doi:10.1371/journal.pone.0053922

78. Zeng K, Wang Y, Ouyang G, Bian Z, Wang L, Li X. Complex network analysis of resting state EEG in amnestic mild cognitive impairment patients with type 2 diabetes. Front Comput Neurosci. 2015;9:133. doi:10.3389/fncom.2015.00133

79. Frantzidis CA, Vivas AB, Tsolaki A, Klados MA, Tsolaki M, Bamidis PD. Functional disorganization of small-world brain networks in mild Alzheimer's disease and amnestic Mild cognitive impairment: An EEG study using Relative Wavelet Entropy (RWE). Front Aging Neurosci. 2014;6:224. doi:10.3389/fnagi.2014.00224

80. Koenig T, Prichep L, Dierks T, et al. Decreased EEG synchronization in Alzheimer's disease and mild cognitive impairment. Neurobiol Aging. 2005;26(2):165-171. doi:10.1016/j.neurobiolaging.2004.03.008

81. Pineda-Pardo JA, Garcés P, López ME, et al. White Matter Damage Disorganizes Brain Functional Networks in Amnestic Mild Cognitive Impairment. Brain Connect. 2014;4(5):312-322. doi:10.1089/brain.2013.0208

82. Gomez C, Stam CJ, Hornero R, Fernandez A, Maestu F. Disturbed Beta Band Functional Connectivity in Patients With Mild Cognitive Impairment: An MEC Study. IEEE Trans Biomed Eng. 2009;56(6):1683-1690. doi:10.1109/TBME.2009.2018454

83. López-Sanz D, Bruña R, Garcés P, et al. Functional Connectivity Disruption in Subjective Cognitive Decline and Mild Cognitive Impairment: A Common Pattern of Alterations. Front Aging Neurosci. 2017;9:109. doi:10.3389/fnagi.2017.00109 
84. Achard S, Delon-Martin C, Vertes PE, et al. Hubs of brain functional networks are radically reorganized in comatose patients. Proc Nat/ Acad Sci. 2012;109(50):20608 20613. doi:10.1073/pnas.1208933109

85. Crone JS, Soddu A, Höller Y, et al. Altered network properties of the fronto-parietal network and the thalamus in impaired consciousness. Neurolmage Clin. 2014;4:240 248. doi:10.1016/j.nicl.2013.12.005

86. van Montfort SJT, van Dellen E, van den Bosch AMR, et al. Resting-state fMRI reveals network disintegration during delirium. Neurolmage Clin. 2018;20:35-41. doi:10.1016/J. NICL.2018.06.024

87. Chen H-J, Chen Q-F, Yang Z-T, Shi H-B. Aberrant topological organization of the functional brain network associated with prior overt hepatic encephalopathy in cirrhotic patients. Brain Imaging Behav. May 2018. doi:10.1007/s11682-018-9896-y

88. Brandt SA, Kraft A, Traenkner S, Schreiber SJ, Fleischmann R, Schmidt S. Delirium is associated with frequency band specific dysconnectivity in intrinsic connectivity networks: preliminary evidence from a large retrospective pilot case-control study. Pilot Feasibility Stud. 2019;5(1):1-13. doi:10.1186/s40814-018-0388-z

89. Numan T, Slooter AJC, van der Kooi AW, et al. Functional connectivity and network analysis during hypoactive delirium and recovery from anesthesia. Clin Neurophysiol. 2017;128(6):914-924. doi:10.1016/j.clinph.2017.02.022

90. He Y, Chen Z, Evans A. Structural Insights into Aberrant Topological Patterns of LargeScale Cortical Networks in Alzheimer's Disease.J Neurosci. 2008;28(18):4756-4766. doi:10.1523/JNEUROSCI.0141-08.2008

91. Liu Y, Yu C, Zhang X, et al. Impaired long distance functional connectivity and weighted network architecture in Alzheimer's disease. Cereb Cortex. 2014;24(6):1422-1435. doi:10.1093/cercor/bhs410

92. Tijms BM, Möller C, Vrenken H, et al. Single-Subject Grey Matter Graphs in Alzheimer's Disease. He Y, ed. PLoS One. 2013;8(3):e58921. doi:10.1371/journal.pone.0058921

93. John M, Ikuta T, FerbinteanuJ. Graph analysis of structural brain networks in Alzheimer's disease: beyond small world properties. Brain Struct Funct. 2017;222(2):923-942. doi:10.1007/s00429-016-1255-4

94. Wang T, Shi F, Jin Y, et al. Multilevel Deficiency of White Matter Connectivity Networks in Alzheimer's Disease: A Diffusion MRI Study with DTI and HARDI Models. Neural Plast. 2016;2016. doi:10.1155/2016/2947136

95. Lo C, Wang P-N, Chou K, Wang J, He Y, Lin C-P. Diffusion Tensor Tractography Reveals Abnormal Topological Organization in Structural Cortical Networks in Alzheimer's Disease.J Neurosci. 2010;30(50):16876-16885. doi:10.1523/JNEUROSCI.4136-10.2010
96. Reijmer YD, Leemans A, Caeyenberghs K, et al. Disruption of cerebral networks and cognitive impairment in Alzheimer disease. Neurology. 2013;80(15):1370-1377. doi:10.1212/WNL.0b013e31828c2ee5

97. Agosta F, Sala S, Valsasina P, et al. Brain network connectivity assessed using graph theory in frontotemporal dementia. Neurology. 2013;81(2):134-143. doi:10.1212/ WNL.0b013e31829a33f8

98. Sanz-Arigita EJ, Schoonheim MM, DamoiseauxJS, et al. Loss of "Small-World" Networks in Alzheimer's Disease: Graph Analysis of $\mathrm{fMRI}$ Resting-State Functional Connectivity. S. Breitner JC, ed. PLoS One. 2010;5(11):e13788. doi:10.1371/journal.pone.0013788

99. Zhao X, Liu Y, Wang X, et al. Disrupted Small-World Brain Networks in Moderate Alzheimer's Disease: A Resting-State fMRI Study. Sporns O, ed. PLoS One. 2012;7(3):e33540. doi:10.1371/journal.pone.0033540

100. Supekar K, Menon V, Rubin D, Musen M, Greicius MD. Network analysis of intrinsic functional brain connectivity in Alzheimer's disease. PLoS Comput Biol. 2008;4(6):e1000100. doi:10.1371/journal.pcbi.1000100

101. Qin Y, Li Y, Zhang S, et al. Frequency-specific alterations of large-scale functional brain networks in patients with Alzheimer's disease. Chin MedJ (Engl). 2015;128(5):602-609. doi:10.4103/0366-6999.151654

102. Peraza LR, Taylor J-P, Kaiser M. Divergent brain functional network alterations in dementia with Lewy bodies and Alzheimer's disease. Neurobio/Aging. 2015;36(9):2458 2467. doi:10.1016/j.neurobiolaging.2015.05.015

103. Filippi M, Basaia S, Canu E, et al. Brain network connectivity differs in early-onset neurodegenerative dementia. Neurology. 2017;89(17):1764-1772. doi:10.1212/ WNL.0000000000004577

104. de Haan W, Pijnenburg Y AL, Strijers RL, et al. Functional neural network analysis in frontotemporal dementia and Alzheimer's disease using EEG and graph theory. BMC Neurosci. 2009;10(1):101. doi:10.1186/1471-2202-10-101

105. Stam CJ, Jones BF, Nolte G, Breakspear M, Scheltens P. Small-world networks and functional connectivity in Alzheimer's disease. Cereb Cortex. 2007;17(1):92-99. doi:10.1093/cercor/bhj127

106. van Dellen E, de Waal H, van der Flier WM, et al. Loss of EEGNetwork Efficiency Is Related to Cognitive Impairment in Dementia With Lewy Bodies. Mov Disord. 2015;30(13):1785-1793. doi:10.1002/mds.26309

107. Afshari S, Jalili M. Directed Functional Networks in Alzheimer's Disease: Disruption of Global and Local Connectivity Measures. IEEE J Biomed Heal Informatics. 2017;21(4):949-955. doi:10.1109/JBHI.2016.2578954 
108. Berendse HW, de Munck JC, Scheltens P, et al. Graph theoretical analysis of magnetoencephalographic functional connectivity in Alzheimer's disease. Brain 2008;132(1):213-224. doi:10.1093/brain/awn262

109. De Haan W, Van der Flier WM, Koene T, Smits LL, Scheltens P, Stam CJ. Disrupted modular brain dynamics reflect cognitive dysfunction in Alzheimer's disease. Neuroimage. 2012;59(4):3085-3093. doi:10.1016/j.neuroimage.2011.11.055

110. Ajilore O, Lamar M, Kumar A. Association of Brain Network Efficiency with Aging, Depression, and Cognition. AmJ Geriatr Psychiatry. 2014;22(2):102-110. doi:10.1016/J. JAGP.2013.10.004

111. Lim HK, Jung WS, Aizenstein HJ. Aberrant topographical organization in gray matter structural network in late life depression: a graph theoretical analysis. Int Psychogeriatrics. 2013;25(12):1929-1940. doi:10.1017/S104161021300149X

112. Singh MK, Kesler SR, Hadi Hosseini SM, et al. Anomalous Gray Matter Structura Networks in Major Depressive Disorder. Biol Psychiatry. 2013;74(10):777-785. doi:10.1016/j.biopsych.2013.03.005

113. Lee CU, Lim HK, Shin J-H, Seong J-K, Um YH. Multiple cortical thickness subnetworks and cognitive impairments in first episode, drug naive patients with late life depression: A graph theory analysis.J Affect Disord. 2018;229:538-545. doi:10.1016/j. jad.2017.12.083

114. Chen VCH, Shen CY, Liang SHY, et al. Assessment of abnormal brain structures and networks in major depressive disorder using morphometric and connectome analyses. J Affect Disord. 2016;205:103-111. doi:10.1016/j.jad.2016.06.066

115. Mak E, Colloby SJ, Thomas A, O'Brien JT. The segregated connectome of late-life depression: a combined cortical thickness and structural covariance analysis. Neurobio/ Aging. 2016;48:212-221. doi:10.1016/j.neurobiolaging.2016.08.013

116. Korgaonkar MS, Fornito A, Williams LM, Grieve SM. Abnormal structural networks characterize major depressive disorder: A connectome analysis. Biol Psychiatry 2014;76(7):567-574. doi:10.1016/j.biopsych.2014.02.018

117. Ajilore O, Lamar M, Leow A, Zhang A, Yang S, Kumar A. Graph theory analysis of corticalsubcortical networks in late-life depression. Am J Geriatr Psychiatry. 2014;22(2):195 206. doi:10.1016/j.jagp.2013.03.005

118. Qin J, Wei M, Liu H, et al. Abnormal brain anatomical topological organization of the cognitive-emotional and the frontoparietal circuitry in major depressive disorder. Magn Reson Med. 2014;72(5):1397-1407. doi:10.1002/mrm.25036

119. Charlton RA, Leow A, Gadelkarim J, et al. Brain Connectivity in Late-Life Depression and Aging Revealed by Network Analysis. Am J Geriatr Psychiatry. 2015;23(6):642-650. doi:10.1016/j.jagp.2014.07.008
120. Nigro S, Passamonti L, Riccelli R, et al. Structural "connectomic" alterations in the limbic system of multiple sclerosis patients with major depression. Mult Scler J. 2015;21(8):1003-1012. doi:10.1177/1352458514558474

121. Long Z, Duan X, Wang Y, et al. Disrupted structural connectivity network in treatmentnaive depression. Prog Neuro-Psychopharmacology Biol Psychiatry. 2015;56:18-26. doi:10.1016/j.pnpbp.2014.07.007

122. Chen JH, Yao ZJ, Qin JL, Yan R, Hua LL, Lu Q. Aberrant global and regional topological organization of the fractional anisotropy-weighted brain structural networks in major depressive disorder. Chin Med J (Engl). 2016;129(6):679-689. doi:10.4103/0366 6999.178002

123. Bohr IJ, Kenny E, Blamire A, et al. Resting-state functional connectivity in late-life depression: Higher global connectivity and more long distance connections. Front Psychiatry. 2013;3:116. doi:10.3389/fpsyt.2012.00116

124. Meng C, Brandl F, Tahmasian M, et al. Aberrant topology of striatum's connectivity is associated with the number of episodes in depression. Brain. 2014;137(2):598-609. doi:10.1093/brain/awt290

125. Lord A, Horn D, Breakspear M, Walter M. Changes in Community Structure of Resting State Functional Connectivity in Unipolar Depression. Zang Y-F, ed. PLoS One. 2012;7(8):e41282. doi:10.1371/journal.pone.0041282

126. Luo Q, Deng Z, Qin J, et al. Frequency dependant topological alterations of intrinsic functional connectome in major depressive disorder. Sci Rep. 2015;5:9710. doi:10.1038/ srep09710

127. Zhang J, Wang J, Wu Q, et al. Disrupted Brain Connectivity Networks in Drug-Naive, First-Episode Major Depressive Disorder. Biol Psychiatry. 2011;70(4):334-342. doi:10.1016/j.biopsych.2011.05.018

128. Jin C, Gao C, Chen C, et al. A preliminary study of the dysregulation of the resting networks in first-episode medication-naive adolescent depression. Neurosci Lett. 2011;503(2):105-109. doi:10.1016/j.neulet.2011.08.017

129. Ye M, Yang T, Qing P, Lei X, Qiu J, Liu G. Changes of Functional Brain Networks in Major Depressive Disorder: A Graph Theoretical Analysis of Resting-State fMRI. Hayasaka S, ed. PLoS One. 2015;10(9):e0133775. doi:10.1371/journal.pone.0133775

130. Wang Z, Yuan Y, Bai F, You J, Zhang Z. Altered topological patterns of brain networks in remitted late-onset depression: a resting-state fMRI study. J Clin Psychiatry. 2016;77(1):123-130. doi:10.4088/JCP.14m09344

131. Ye M, Qing P, Zhang K, Liu G. Altered network efficiency in major depressive disorder. BMC Psychiatry. 2016;16(1):450. doi:10.1186/s12888-016-1053-9 
132. Leuchter AF, Cook IA, Hunter AM, Cai C, Horvath S. Resting-State Quantitative Electroencephalography Reveals Increased Neurophysiologic Connectivity in Depression. El-Deredy W, ed. PLoS One. 2012;7(2):e32508. doi:10.1371/journal. pone.0032508

133. Shim M, Im CH, Kim YW, Lee SH. Altered cortical functional network in major depressive disorder: A resting-state electroencephalogram study. Neurolmage Clin. 2018;19:1000-1007. doi:10.1016/j.nicl.2018.06.012

134. Zhang LJ ian., Zheng G, Zhang L, et al. Disrupted small world networks in patients without overt hepatic encephalopathy: a resting state fMRI study. Eur J Radiol 2014;83(10):1890-1899. doi:10.1016/j.ejrad.2014.06.019

135. Jao T, Schröter M, Chen C-L, et al. Functional brain network changes associated with clinical and biochemical measures of the severity of hepatic encephalopathy. Neuroimage. 2015;122:332-344. doi:10.1016/j.neuroimage.2015.07.068

136. Li Q, Shi D, Zheng G, et al. Altered Modular Organization of Functional Connectivity Networks in Cirrhotic Patients without Overt Hepatic Encephalopathy. Biomed Res Int. 2014;2014:1-11. doi:10.1155/2014/727452

137. Kim E, Kang H, Lee HH-J, et al. Morphological brain network assessed using graph theory and network filtration in deaf adults. Hear Res. 2014;315:88-98. doi:10.1016/j. heares.2014.06.007

138. Xu H, Fan W, Zhao X, et al. Disrupted functional brain connectome in unilateral sudden sensorineural hearing loss. Hear Res. 2016;335:138-148. doi:10.1016/j. heares.2016.02.016

139. Zhang $Y$, Mao Z, Feng $S$, et al. Altered functional networks in long-term unilateral hearing loss: A connectome analysis. Brain Behav. 2018;8(2):e00912. doi:10.1002/ brb3.912

140. Wang L, Xia M, Li K, et al. The effects of antidepressant treatment on resting-state functional brain networks in patients with major depressive disorder. Hum Brain Mapp. 2015;36(2):768-778. doi:10.1002/hbm.22663

141. Ma X, Jiang G, Li S, et al. Aberrant Functional Connectome in Neurologically Asymptomatic Patients with End-Stage Renal Disease. Hayasaka S, ed. PLoS One. 2015;10(3):e0121085. doi:10.1371/journal.pone.0121085

142. Monti MM, Lutkenhoff ES, Rubinov M, et al. Dynamic Change of Global and Loca Information Processing in Propofol-Induced Loss and Recovery of Consciousness. Daunizeau J, ed. PLoS Comput Biol. 2013;9(10):e1003271. doi:10.1371/journal. pcbi.100327
143. Hashmi JA, Loggia ML, Khan S, et al. Dexmedetomidine Disrupts the Local and Global Efficiencies of Large-scale Brain Networks. Anesthesiology. 2017;126(3):419-430. doi:10.1097/ALN.0000000000001509

144. Lee H, Mashour GA, Noh G-J, Kim S, Lee U. Reconfiguration of network hub structure after propofol-induced unconsciousness. Anesthesiology. 2013;119(6):1347-1359. doi:10.1097/ALN.0b013e3182a8ec8c

145. Blain-Moraes S, Tarnal V, Vanini G, et al. Network Efficiency and Posterior Alpha Patterns Are Markers of Recovery from General Anesthesia: A High-Density Electroencephalography Study in Healthy Volunteers. Front Hum Neurosci. 2017;11:328. doi:10.3389/fnhum.2017.00328

146. Shi L, Wang D, Chu WCW, et al. Abnormal Organization of White Matter Network in Patients with No Dementia after Ischemic Stroke. Zuo X-N, ed. PLoS One. 2013;8(12):e81388. doi:10.1371/journal.pone.0081388

147. Guo X, Jin Z, Feng X, Tong S. Enhanced effective connectivity in mild occipital stroke patients with hemianopia. IEEE Trans Neural Syst Rehabil Eng. 2014;22(6):1210-1217. doi:10.1109/TNSRE.2014.2325601

148. Kim J, Parker D, Whyte J, et al. Disrupted Structural Connectome Is Associated with Both Psychometric and Real-World Neuropsychological Impairment in Diffuse Traumatic Brain Injury. J Int Neuropsychol Soc. 2014;20(9):887-896. doi:10.1017/ S1355617714000812

149. Caeyenberghs K, Leemans A, Leunissen I, et al. Altered structural networks and executive deficits in traumatic brain injury patients. Brain Struct Funct. 2014;219(1):193-209. doi:10.1007/s00429-012-0494-2

150. Messé A, Caplain S, Pélégrini-Issac M, et al. Specific and Evolving Resting-State Network Alterations in Post-Concussion Syndrome Following Mild Traumatic Brain Injury. Valdes-Sosa PA, ed. PLoS One. 2013;8(6):e65470. doi:10.1371/journal. pone. 0065470

151. Han K, Mac Donald CL, Johnson AM, et al. Disrupted modular organization of resting state cortical functional connectivity in U.S. military personnel following concussive "mild" blast-related traumatic brain injury. Neuroimage. 2014;84:76-96. doi:10.1016/j. neuroimage.2013.08.017

152. van der Horn HJ, Liemburg EJ, Scheenen ME, de Koning ME, Spikman JM, van der Naalt J. Graph Analysis of Functional Brain Networks in Patients with Mild Traumatic Brain Injury. Stamatakis EA, ed. PLoS One. 2017;12(1):e0171031. doi:10.1371/journal. pone. 0171031 
153. Maestú F, Boccaletti S, Castellanos NP, et al. Principles of recovery from traumatic brain injury: Reorganization of functional networks. Neuroimage. 2010;55(3):1189 1199. doi:10.1016/j.neuroimage.2010.12.046

154. Shu N, Liu Y, Li J, Li Y, Yu C, Jiang T. Altered Anatomical Network in Early Blindness Revealed by Diffusion Tensor Tractography. Sporns O, ed. PLoS One. 2009;4(9):e7228. doi:10.1371/journal.pone.0007228

155. Bola M, Gall C, Moewes C, Fedorov A, Hinrichs H, Sabel BA. Brain functional connectivity network breakdown and restoration in blindness. Neurology. 2014;83(6):542-551. doi:10.1212/WNL.0000000000000672

156. Wang L, Guo X, Sun J, Jin Z, Tong S. Cortical networks of hemianopia stroke patients: a graph theoretical analysis of EEG signals at resting state. Eng Med Biol Soc. 2012;2012:49-52. doi:10.1109/EMBC.2012.6345868

157. Disorder FMD, Zhang J, Wang J, et al. Disrupted Brain Connectivity Networks in Drug Naive ,. Biol Psychiatry. 2011;70(4):334-342. doi:10.1016/j.biopsych.2011.05.018

158. Tewarie P, van Dellen E, Hillebrand A, Stam CJ. The minimum spanning tree: An unbiased method for brain network analysis. Neuroimage. 2015;104:177-188. doi:10.1016/j. neuroimage.2014.10.015

159. Stam CJ, Tewarie P, Van Dellen E, van Straaten ECW, Hillebrand A, Van Mieghem P. The trees and the forest: Characterization of complex brain networks with minimum spanning trees. Int J Psychophysiol. 2014;92(3):129-138. doi:10.1016/j. ijpsycho.2014.04.001

160. Fried El, Nesse RM, Zivin K, Guille C, Sen S. Depression is more than the sum score of its parts: individual DSM symptoms have different risk factors. Psychol Med. 2014;44(10):2067-2076. doi:10.1017/S0033291713002900

161. Kraft RH, Mckee PJ, Dagro AM, Grafton ST. Combining the Finite Element Method with Structural Connectome-based Analysis for Modeling Neurotrauma: Connectome Neurotrauma Mechanics. Sporns O, ed. PLoS Comput Biol. 2012;8(8):e1002619. doi:10.1371/journal.pcbi.1002619

162. Mashour, GA, Hudetz A. Neural Correlates of Unconsciousness in Large-Scale Brain Networks. Trends Neurosci. 2018;41(3):150-160.

163. Easterbrook P., Gopalan R, Berlin J., Matthews D. Publication bias in clinical research. Lancet. 1991;337(8746):867-872. doi:10.1016/0140-6736(91)90201-Y

164. Rothstein HR, Sutton AJ, Borenstein M. Publication Bias in Meta-Analysis: Prevention, Assessment and Adjustments. In: Wiley; 2006:374. doi:10.1002/0470870168.ch1

165. H. Roder C, Marie Hoogendam J, M. van der Veen F. FMRI, Antipsychotics and Schizophrenia. Influence of Different Antipsychotics on BOLD-Signal. Curr Pharm Des. 2010;16(18):2012-2025. doi:10.2174/138161210791293088
166. Kant IMJ, de Bresser J, van Montfort SJT, Slooter AJC, Hendrikse J. MRI Markers of Neurodegenerative and Neurovascular Changes in Relation to Postoperative Delirium and Postoperative Cognitive Decline. Am J Geriatr Psychiatry. 2017;25(10) doi:10.1016/j.jagp.2017.06.016

167. Honey CJ, Sporns O, Cammoun L, et al. Predicting human resting-state functional connectivity from structural connectivity. Proc Nat/ Acad Sci U SA. 2009;106(6):20352040. doi:10.1073/pnas.0811168106

168. Cabral J, Hugues E, Kringelbach ML, Deco G. Modeling the outcome of structural disconnection on resting-state functional connectivity. Neuroimage. 2012;62(3):1342 1353. doi:10.1016/j.neuroimage.2012.06.007

169. Cabral J, Kringelbach M, Deco G. Functional Graph Alterations in Schizophrenia: A Result from a Global Anatomic Decoupling? Pharmacopsychiatry. 2012;45(S 01):S57S64. doi:10.1055/s-0032-1309001

170. Mišić B, Betzel RF, Nematzadeh A, et al. Cooperative and Competitive Spreading Dynamics on the Human Connectome. Neuron. 2015;86(6):1518-1529. doi:10.1016/j. neuron.2015.05.035

171. Kyeong S, Shin JE, Yang KH, Lee WS, Chung T-S, Kim J-J. Neural predisposing factors of postoperative delirium in elderly patients with femoral neck fracture. Sci Rep. 2018;8(1):7602. doi:10.1038/s41598-018-26030-2

172. Chen H-J, Shi H-B, Jiang L-F, Li L, Chen R. Disrupted topological organization of brain structural network associated with prior overt hepatic encephalopathy in cirrhotic patients. Eur Radiol. 2018;28(1):85-95. doi:10.1007/s00330-017-4887-8

173. Maldonado JR. Delirium pathophysiology: An updated hypothesis of the etiology of acute brain failure. Int J Geriatr Psychiatry. 2018;33(11):1428-1457. doi:10.1002/ gps.4823

174. Ponten SC, Tewarie P, Slooter AJC, Stam CJ, van Dellen E. Neural Network Modeling of EEG Patterns in Encephalopathy.J Clin Neurophysio/. 2013;30(5):545-552. doi:10.1097/ WNP.0b013e3182a73e16

175. Ferrarelli F, Massimini M, Sarasso S, et al. Breakdown in cortical effective connectivity during midazolam-induced loss of consciousness. Proc Natl Acad Sci U S A 2010;107(6):2681-2686. doi:10.1073/pnas.0913008107

176. Cocchi L. Personalized Transcranial Magnetic Stimulation in Psychiatry. Biol Psychiatry Cogn Neurosci Neuroimaging. 2018;3(9):731-741. doi:10.1016/J.BPSC.2018.01.008

177. Kuo M-F, Paulus W, Nitsche MA. Therapeutic effects of non-invasive brain stimulation with direct currents (tDCS) in neuropsychiatric diseases. Neuroimage. 2014;85:948960. doi:10.1016/J.NEUROIMAGE.2013.05.117 


\section{Supplementary Information}

Table S1: PubMed search term $(A+B+C)$

\begin{tabular}{|c|c|c|}
\hline $\begin{array}{l}\text { Part A } \\
\text { (connectivity } \\
\text { OR "network" } \\
\text { OR graph OR } \\
\text { disconnection } \\
\text { OR dyscon- } \\
\text { nection) OR } \\
\text { connectome } \\
\text { [Mesh] OR } \\
\text { connectomics } \\
\text { AND }\end{array}$ & $\begin{array}{l}\text { Part B } \\
\text { delirium [Mesh] OR "post- } \\
\text { operative delirium" OR } \\
\text { "history of delirium } \\
\text { "encephalopathy" } \\
\text { dementia } \\
\text { "cognitive impairment" OR } \\
\text { "cognitive decline" } \\
\text { "functional impairment" } \\
\text { vision disorders [Mesh] } \\
\text { hearing loss [Mesh] } \\
\text { comorbidity [Mesh] } \\
\text { "severity of illness" } \\
\text { frailty } \\
\text { depression [Mesh] } \\
\text { ischemic attack, transient } \\
\text { [Mesh] } \\
\text { "history of transient isch- } \\
\text { emia" } \\
\text { stroke [Mesh] } \\
\text { "history of stroke" } \\
\text { ("alcohol misuse" OR "alco- } \\
\text { hol abuse") } \\
\text { (aging [Mesh] OR "elder- } \\
\text { ly") } \\
\text { (polypharmacy [Mesh] OR } \\
\text { "several drugs") } \\
\text { psychoactive drug [Mesh] } \\
\text { hypnotics and sedatives } \\
\text { [Mesh] } \\
\text { physical restraint [Mesh] } \\
\text { (urinary catheters [Mesh] } \\
\text { OR urea [Mesh]) }\end{array}$ & $\begin{array}{l}\text { Part C } \\
\text { (magnetic resonance imaging } \\
\text { [Mesh] OR electroencephalog- } \\
\text { raphy [Mesh] OR electrocorti- } \\
\text { cography [Mesh] OR diffusion } \\
\text { tensor imaging [Mesh] OR } \\
\text { "resting state" OR magneto- } \\
\text { encephalography [Mesh] OR } \\
\text { brain [Mesh] OR neuroimaging } \\
\text { [Mesh] OR functional neuroim- } \\
\text { aging [Mesh] OR positron-emis- } \\
\text { sion tomography [Mesh] OR } \\
\text { staining [Mesh] OR neurophysi- } \\
\text { ology [Mesh] OR diffusion trac- } \\
\text { tography [Mesh] OR diffusion } \\
\text { magnetic resonance imaging } \\
\text { [Mesh] OR "spectroscopy, near } \\
\text { infrared" [Mesh]) }\end{array}$ \\
\hline
\end{tabular}

Table S1: PubMed search term $(A+B+C)$ (continued)

Part A

$$
\text { Part B }
$$

(blood urea nitrogen [Mesh] OR creatinine$$
\text { [Mesh]) }
$$

("renal failure" OR renal insufficiency [Mesh] OR acute kidney injury [Mesh])

albumins [Mesh]

(hyponatremia [Mesh] OR hypernatremia [Mesh])

(hyperglycemia [Mesh] OR hypoglycaemia [Mesh]) glucose [Mesh]

(hyperkalemia [Mesh] OR hypokalemia [Mesh])

("metabolic acidosis" OR acidosis [Mesh])

infection [Mesh]

("iatrogenic event" OR iatrogenic disease (Mesh))

aortic aneurysm [Mesh] surgery [Mesh]

("trauma" OR wounds and injuries [Mesh]) NOT stress disorders, post-traumatic [Mesh]

(urgent OR ambulatory care [Mesh])

coma [Mesh] 
Table S2: EMBASE search term $(A+B+C)$

\begin{tabular}{l|l} 
Part A & Part B \\
('connectome'/exp & 'delirium'/exp OR \\
'graph'OR' 'd?s- & 'postoperative de- \\
connection') AND & lirium' OR 'history of \\
delirium' \\
encephalopathy \\
& 'dementia' NOT \\
'mild cognitive im- \\
pairment' \\
'cognitive impair- \\
ment' NOT ('de- \\
mentia'/exp OR \\
'dementia') OR \\
'cognitive decline' \\
'functional impair- \\
ment' \\
'visual disorder' \\
'hearing loss' \\
comorbidity'/exp \\
'severity of illness' \\
'frailty' \\
'depression'/exp \\
'transient ischemic \\
attack' \\
'history of transient \\
ischemia' \\
'stroke'/exp OR 'his- \\
tory of stroke' \\
'alcohol misuse' OR \\
'alcohol abuse' \\
'aging'/exp NOT \\
'dementia' NOT \\
'dementia'/exp \\
'polypharmacy' OR \\
'several drugs used' \\
'psychoactive drug' \\
\end{tabular}

Table S2: EMBASE search term $(A+B+C)$ (continued)

Part C

'nuclear magnetic resonance

imaging'/exp or 'diffusion tensor

imaging'/exp or 'magnetoenceph-

alography system'/exp or 'electro-

encephalogram'/exp or 'brain'/exp

or 'neuroimaging'/exp or 'functional

neuroimaging'/exp or 'positron

emission tomography'/exp or

'staining'/exp or 'neurophysiology'/

exp or 'diffusion weighted imaging'/

exp or 'near infrared spectroscopy'/

exp) and [humans]/lim and [en-

glish]/lim and [abstracts]/lim
Part A

\begin{tabular}{|l|l} 
Part B & Part C \\
'hypnotic agent' OR & \\
'sedative agent' & \\
'physical restraint' & \\
'urinary catheter' & \\
OR 'urea'/exp \\
'blood urea nitro- \\
gen' OR 'creatinine'/ \\
exp \\
'renal failure'/exp \\
OR 'acute kidney \\
injury'/exp \\
'albumins' \\
'hyponatremia' OR \\
'hypernatremia' \\
'hyperglycemia' OR \\
'hypoglycaemia' \\
'glucose'/exp \\
'hyperkalemia' OR \\
'hypokalemia' \\
'metabolic acidosis' \\
'infection' \\
'iatrogenic disease' \\
'aorta aneurysm' \\
'surgery/exp \\
'trauma' NOT 'post- \\
traumatic stress \\
disorder' \\
'urgent medical \\
aid' OR 'ambulatory \\
care' \\
'coma'/exp
\end{tabular}




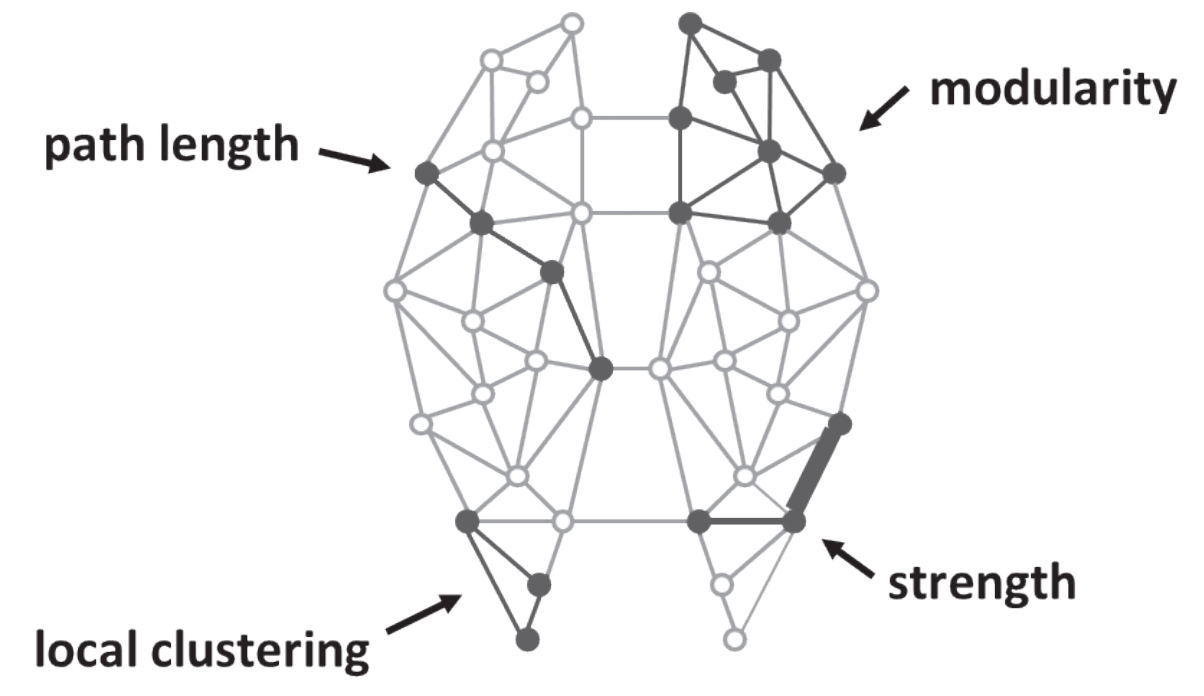

Figure S1 A network or graph consists of nodes, i.e. brain regions, and edges, i.e. the structural or functional connection between brain regions. The strength of a network relates to the strength of the edges and may be used to indicate the overall level of connectivity (indicated in the lower right corner by the thickness of the edges). Graph analyses can then be used to characterize different aspects of network organization. The path length relate to the minimum number of edges or the shortest path between two nodes (illustrated in the upper left corner). This indicates how efficient these nodes are connected, where global efficiency is higher for shorter path lengths. Local clustering (illustrated in the lower left corner) quantifies the fraction of neighbors of a node (i.e. other nodes that it is directly connected with) that are also connected with each other, which is a measure of local segregation. Modularity (upper right corner) characterizes the presence of communities or modules within the full network. Modules may represent functional subunits of a network, for example the visual system, with high connectivity within the community, but low connectivity with nodes outside of the module.

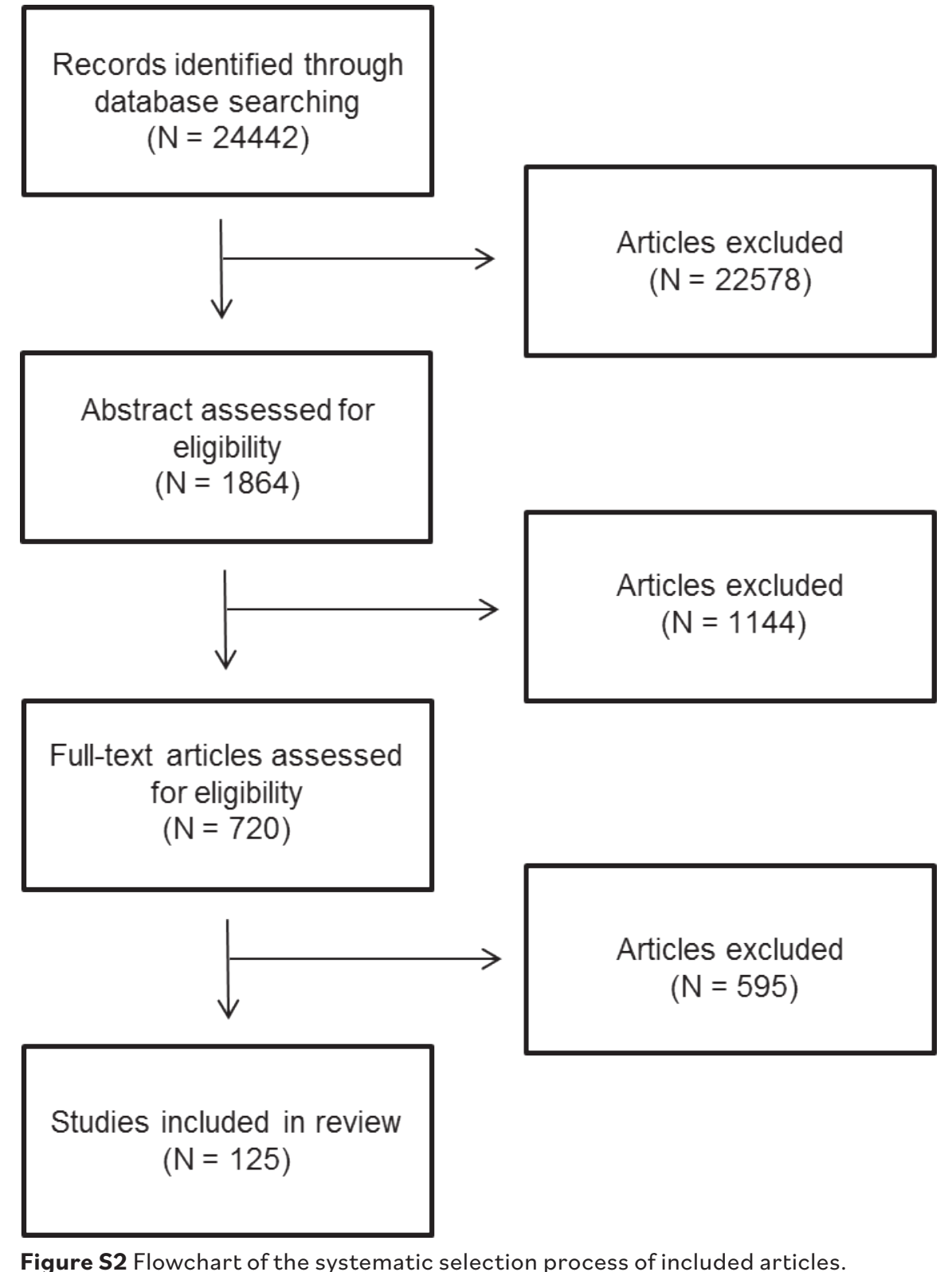

Figure S2 Flowchart of the systematic selection process of included articles. 


\section{Supplementary Information Text Section A1 Explanation of quality criteria}

The quality criteria are displayed in Figure 1, below we explain the separate items.

\section{Part I}

1. Sample size for the smallest group

Sample size is important for the evidence and reliability of the results. Assuming weak to medium effect sizes in network studies, numbers shown in Figure 1 appeared to be required (Friston, 2012; Lenth, 2001).

\section{Definition of patient population}

The use of a validated screening or diagnostic tool or consensus of different experts on the diagnosis is essential to define the patient group properly and to exclude noise based on incorrectly classified patients (van Diessen et al., 2015).

\section{Confounding}

Risk factor specific major confounding variables:

Depression:

Age + gender

Hearing impairment:

Age + gender

Vision impairment:

Cognitive impairment:

Age + gender

Dementia:

Stroke:

IQ/level of education + age

IQ/level of education + age

IQ/level of education + age

Aging:

Coma:

IQ/level of education + gender

Delirium:

Encephalopathy:

Neurotrauma:

Sedation:

representative control cohort + age

representative control cohort + age + gender representative control cohort + age + gender representative control cohort + age + gender within subject comparison to pre-sedative state

Confounders can strongly influence the brain network (Gong et al., 2009), we therefore suggest to control properly for these variables. We focused on risk factors with a known effect on the outcome variables and/or a possible effect on the estimated interaction between the risk factor and the outcome variable.

\section{Part II}

4. Network comparison

Most studies use unweighted, i.e. binary networks. A motivation to use a binary network could be the idea that only important connections are included and to discard spurious connections that are potentially influenced by, for example, noise (Bullmore and Sporns, 2009; van Wijk et al., 2010) Selecting the value for the threshold is, however, arbitrary and may vary between individuals and groups (van Wijk et al., 2010). Therefore, when using an unweighted network, it is suggested to use multiple different thresholds and to adequately correct for multiple testing (Rubinov and Sporns, 2010 van Wijk et al., 2010). A weighted network overcomes the problem of this subjective choice of a threshold and provides a more realistic representation of functional networks, but may include spurious weak connections in the network. In addition, bias is introduced when comparing networks that differ in connectivity values and their distribution, which is often the case when comparing patient groups to healthy controls (van Wijk et al., 2010). Therefore, a regression of connection strength effects (Otte et al., 2015), or a comparison to a null model, e.g. by normalizing the network outcomes by random graphs, is useful (Lee et al., 2010). An alternative adequate network comparison can be performed using the minimum spanning tree (MST). The MST is an acyclic sub-network that connects all nodes and may solve the mentioned methodological limitations of weighted and unweighted networks (Stam et al., 2014; Tewarie et al., 2015)

To increase the transparency of the analysis and its possible influence on the results, the statistics should be reported. To control for type I error rate, multiple comparison correction is recommended (Cao and Zhang, 2014). 


\section{Part III}

5. Methodological quality

a. DTI

Advantages of $3 \mathrm{~T}$ compared to 1,5T are an increased signal to noise ratio, increased spatial resolution and increased temporal resolution (Soher et al., 2007), which may be of importance in defining the edges in DTI network studies (Fornito et al., 2013). There is currently no consensus on the best method to define the edge weights. In accordance with a recent methodological paper we used at least two different methods and show that both lead to similar results (Fornito et al., 2013).

\section{b. $F M R I$}

Longer $\mathrm{fMRI}$ scanning time is preferable, increasing the scan time to at least nine minutes greatly improves the reliability (Birn, R.M., Molloy, E.K., Patriat, R., Parker, T., Meier, T.B., Kirk, G.R., Nair, V.A., Meyerand, M.E., Prabhakaran, 2013). When using PRESTO scans, the duration of scanning does not strongly influence the quality. Since initial reports regarding the strong impact of motion artifact on the reliability of fMRI studies, an effective method of motion correction based on a recently published consensus paper should be applied (Ciric et al., 2017).

\section{c. $E E G / M E G$}

As epoch length and sample frequency can influence the connectivity measure, we recommend to use epochs of an identical length and sample frequency within a study (van Dellen et al., 2014; van Diessen et al., 2015). EEG and MEG techniques are sensitive to field spread, i.e. the problem that multiple electrodes pick up activity from a single source (Sarvas, 1987), and volume conduction, i.e. the 'blurring' effect due to the electrical conduction properties of the brain (van den Broek et al., 1998). Several connectivity measures are identified that correct for both problems which were summarized in a recent methodological paper, we advise to use one of these measures (van Diessen et al., 2015).

\section{Results}

Outcome

Excellent quality

Good quality

DTI, fMRI, EEG studies

8 - 9 points

5 - 7 points

Moderate quality

$0-4$ points

PET, MRI studies

$6-7$ points

4 - 5 points

$0-3$ points

\section{References used for quality criteria:}

Birn, R.M., Molloy, E.K., Patriat, R., Parker, T., Meier, T.B., Kirk, G.R., Nair, V.A., Meyerand, M.E., Prabhakaran, V., 2013. The effect of scan length on the reliability of resting-state fMRI connectivity estimates. Neuroimage 83, 550-558. https://doi.org/10.1016/J NEUROIMAGE.2013.05.099

Bullmore, E., Sporns, O., 2009. Complex brain networks: Graph theoretical analysis of structural and functional systems. Nat. Rev. Neurosci. https://doi.org/10.1038/nrn2575

Cao, J., Zhang, S., 2014. Multiple comparison procedures. JAMA 312, 543-4. https://doi. org/10.1001/jama.2014.9440

Ciric, R., Wolf, D.H., Power, J.D., Roalf, D.R., Baum, G.L., Ruparel, K., Shinohara, R.T., Elliott, M.A., Eickhoff, S.B., Davatzikos, C., Gur, R.C., Gur, R.E., Bassett, D.S., Satterthwaite, T.D., 2017. Benchmarking of participant-level confound regression strategies for the control of motion artifact in studies of functional connectivity. Neuroimage 154, 174187. https://doi.org/10.1016/j.neuroimage.2017.03.020

Fornito, A., Zalesky, A., Breakspear, M., 2013. Graph analysis of the human connectome: Promise, progress, and pitfalls. Neuroimage 80, 426-444. https://doi.org/10.1016/j neuroimage.2013.04.087

Friston, K., 2012. Ten ironic reviews for non-statistical reviewers. Neuroimage 61, 1300 1310.

Gong, G., Rosa-Neto, P., Carbonell, F., Chen, Z.J., He, Y., Evans, A.C., 2009. Age- and genderrelated differences in the cortical anatomical network. J. Neurosci. 29, 15684-93. https://doi.org/10.1523/JNEUROSCI.2308-09.2009

Lee, U., Oh, G., Kim, S., Noh, G., Choi, B., Mashour, G.A., 2010. Brain Networks Maintain a Scale-free Organization across Consciousness, Anesthesia, and Recovery. Anesthesiology 113, 1081-1091. https://doi.org/10.1097/ALN.0b013e3181f229b5

Lenth, R. V, 2001. Some Practical Guidelines for Effective Sample Size Determination. Am. Stat. 55, 187-193. https://doi.org/10.1198/000313001317098149 
Otte, W.M., van Diessen, E., Paul, S., Ramaswamy, R., Subramanyam Rallabandi, V.P., Stam, C.J., Roy, P.K., 2015. Aging alterations in whole-brain networks during adulthood mapped with the minimum spanning tree indices: The interplay of density, connectivity cost and life-time trajectory. Neuroimage 109, 171-189. https://doi.org/10.1016/J. NEUROIMAGE.2015.01.011

Rubinov, M., Sporns, O., 2010. Complex network measures of brain connectivity: Uses and interpretations. Neuroimage 52, 1059-1069. https://doi.org/10.1016/j. neuroimage.2009.10.003

Sarvas, J., 1987. Basic mathematical and electromagnetic concepts of the biomagnetic inverse problem. Phys. Med. Biol. 32, 11-22. https://doi.org/10.1088/0031 9155/32/1/004

Soher, B.J., Dale, B.M., Merkle, E.M., 2007. A review of MR physics: 3 T versus 1.5T. Magn Reson. Imaging Clin. N. Am. 15, 277-90, v. https://doi.org/10.1016/j.mric.2007.06.002

Stam, C.J., Tewarie, P., Van Dellen, E., van Straaten, E.C.W., Hillebrand, A., Van Mieghem, P., 2014. The trees and the forest: Characterization of complex brain networks with minimum spanning trees. Int.J. Psychophysiol. 92, 129-138. https://doi.org/10.1016/j. ijpsycho.2014.04.001

Tewarie, P., van Dellen, E., Hillebrand, A., Stam, C.J., 2015. The minimum spanning tree: An unbiased method for brain network analysis. Neuroimage 104, 177-188. https://doi. org/10.1016/j.neuroimage.2014.10.015

van Dellen, E., van der Kooi, A.W., Numan, T., Koek, H.L., Klijn, F.A.M., Buijsrogge, M.P., Stam, C.J., Slooter, A.J.C., 2014. Decreased Functional Connectivity and Disturbed Directionality of Information Flow in the Electroencephalography of Intensive Care Unit Patients with Delirium after Cardiac Surgery. Anesthesiology 121, 328-335. https://doi.org/10.1097/ALN.0000000000000329

van den Broek, S.., Reinders, F., Donderwinkel, M., Peters, M.., 1998. Volume conduction effects in EEG and MEG. Electroencephalogr. Clin. Neurophysiol. 106, 522-534. https://doi.org/10.1016/S0013-4694(97)00147-8

van den Heuvel, M.P., de Lange, S.C., Zalesky, A., Seguin, C., Yeo, B.T.T., Schmidt, R., 2017. Proportional thresholding in resting-state fMRI functional connectivity networks and consequences for patient-control connectome studies: Issues and recommendations. Neuroimage 152, 437-449. https://doi.org/10.1016/J.NEUROIMAGE.2017.02.005

van Diessen, E., Numan, T., van Dellen, E., van der Kooi, A.W., Boersma, M., Hofman, D., van Lutterveld, R., van Dijk, B.W., van Straaten, E.C.W., Hillebrand, A., Stam, C.J., 2015 Opportunities and methodological challenges in EEG and MEG resting state functional brain network research. Clin. Neurophysiol. 126, 1468-81. https://doi.org/10.1016/j. clinph.2014.11.018 van Wijk, B.C.M., Stam, C.J., Daffertshofer, A., Stam, C., Apkarian, A., 2010. Comparing Brain Networks of Different Size and Connectivity Density Using Graph Theory. PLoS One 5, e13701. https://doi.org/10.1371/journal.pone.0013701 


\section{Text Section A2}

\section{Covariance in efficiency and connectivity}

\section{strength effects}

Since the number of connections and the average connection strength influence network measures, such as efficiency (van den Heuvel et al., 2017; van Wijk et al., 2010) (1,2), some of the observations on decreased efficiency may be explained by loss of connections. An evaluation of this phenomenon in our studies revealed that a decreased efficiency was reported along with a decreased strength in $31 \%$ of the included cases of good or excellent quality. Only $14 \%$ of cases reported decreased efficiency but no decrease in strength, while $66 \%$ of the cases did not provide information on strength differences.

\section{References used for covariance in efficiency and connectivity}

\section{strength effects:}

van den Heuvel, M.P., de Lange, S.C., Zalesky, A., Seguin, C., Yeo, B.T.T., Schmidt, R., 2017 Proportional thresholding in resting-state fMRI functional connectivity networks and consequences for patient-control connectome studies: Issues and recommendations. Neuroimage 152, 437-449. https://doi.org/10.1016/J.NEUROIMAGE.2017.02.005

van Wijk, B.C.M., Stam, C.J., Daffertshofer, A., Stam, C., Apkarian, A., 2010. Comparing Brain Networks of Different Size and Connectivity Density Using Graph Theory. PLoS One 5, e13701. https://doi.org/10.1371/journal.pone.0013701

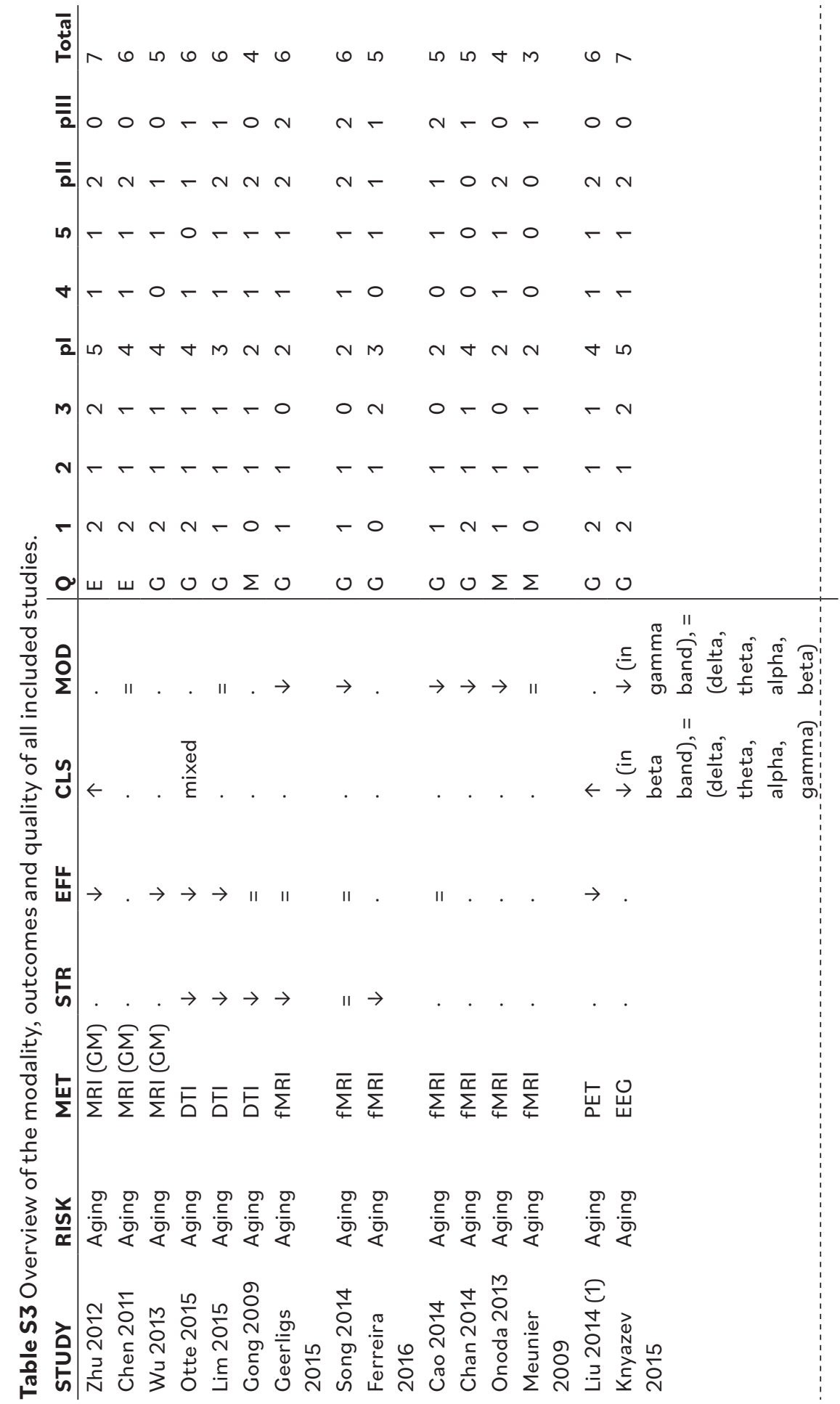



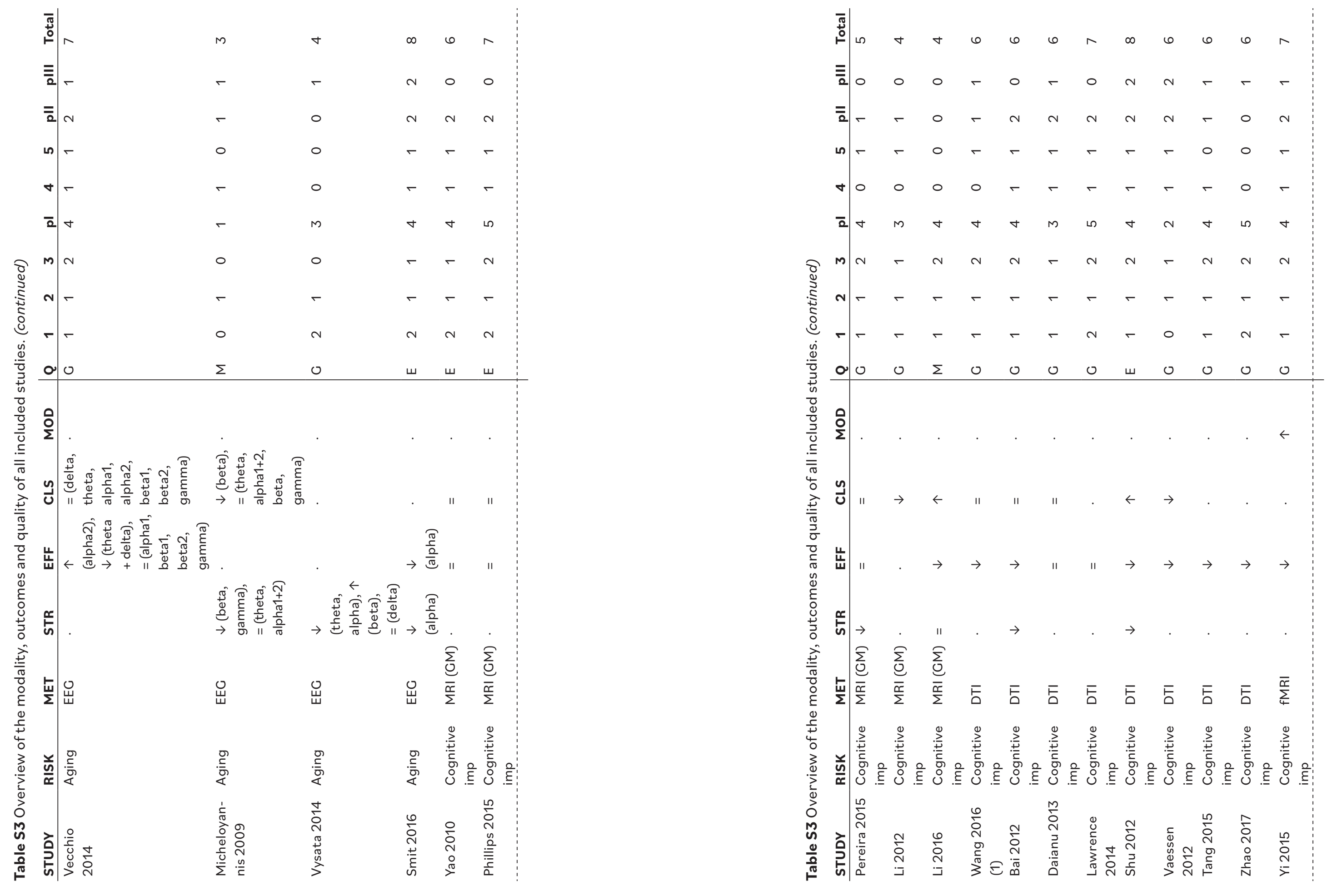

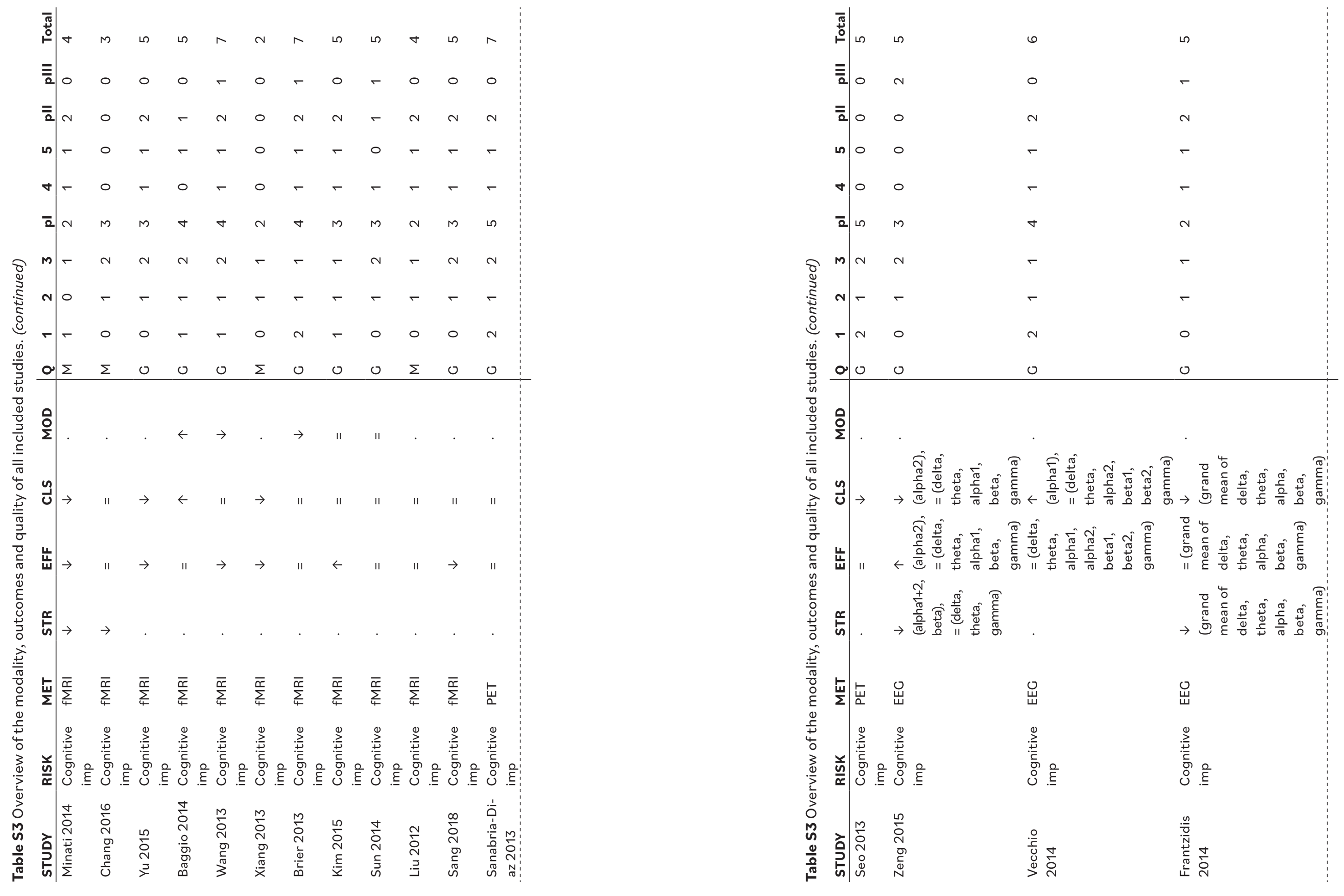

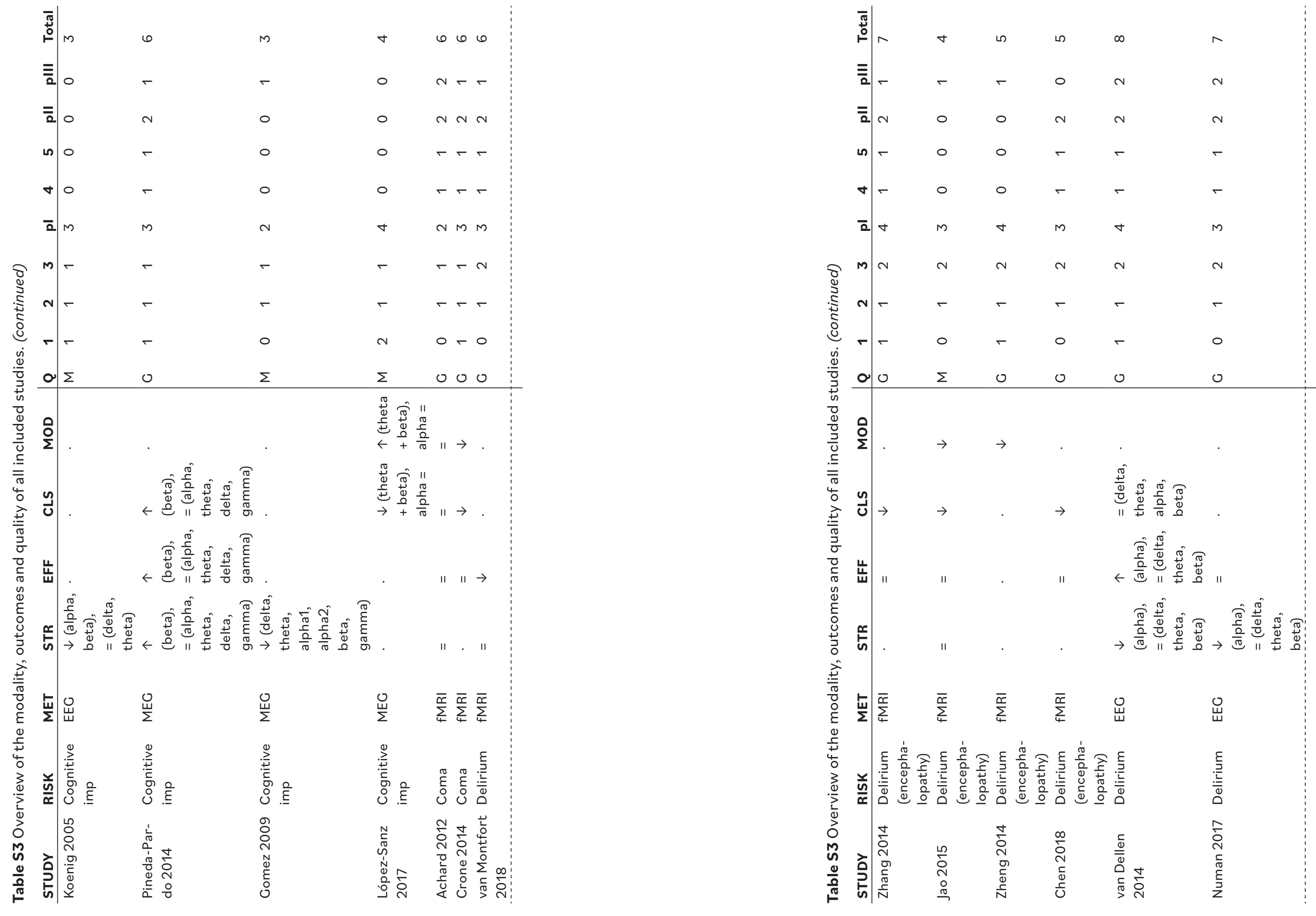

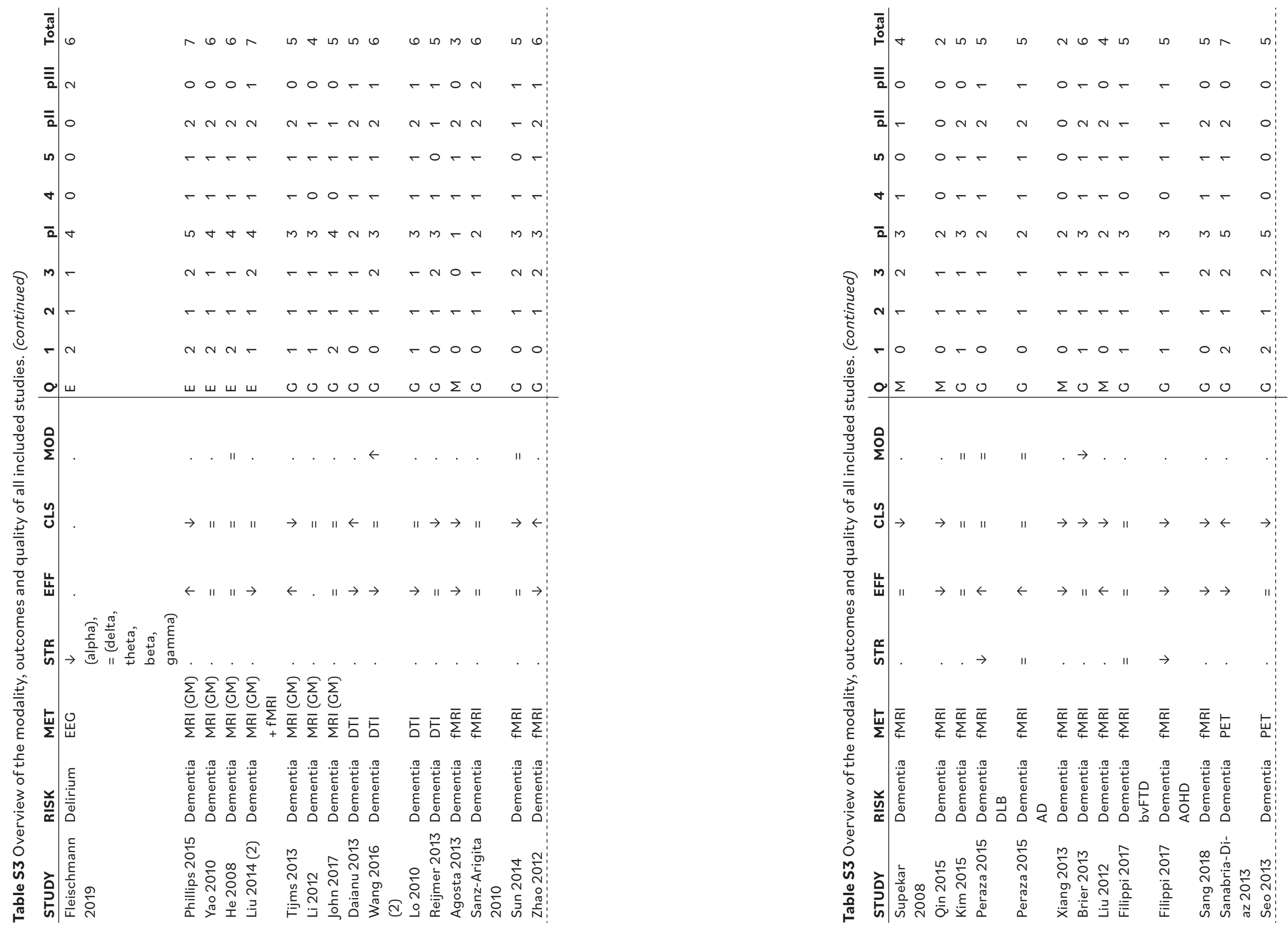

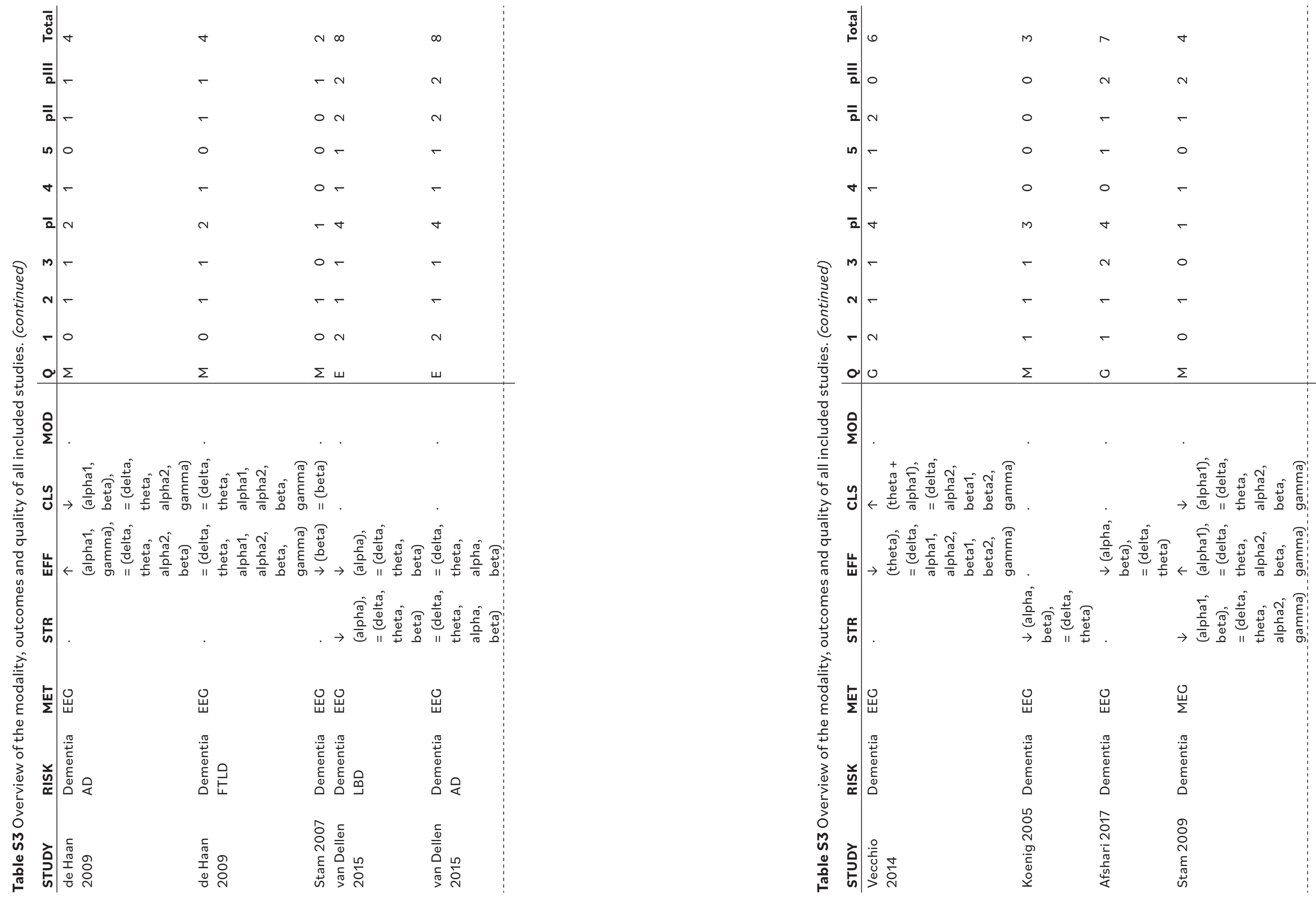

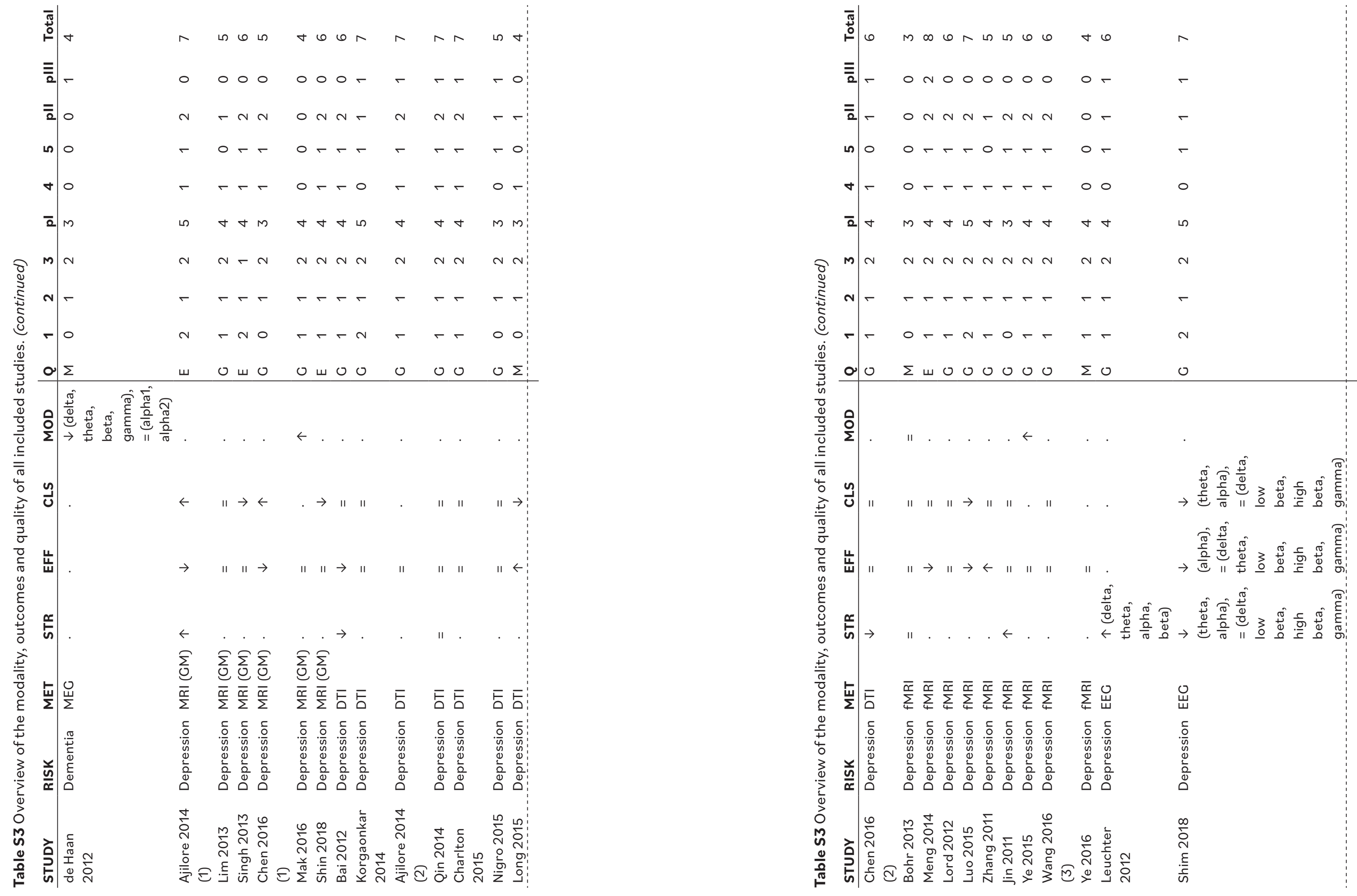

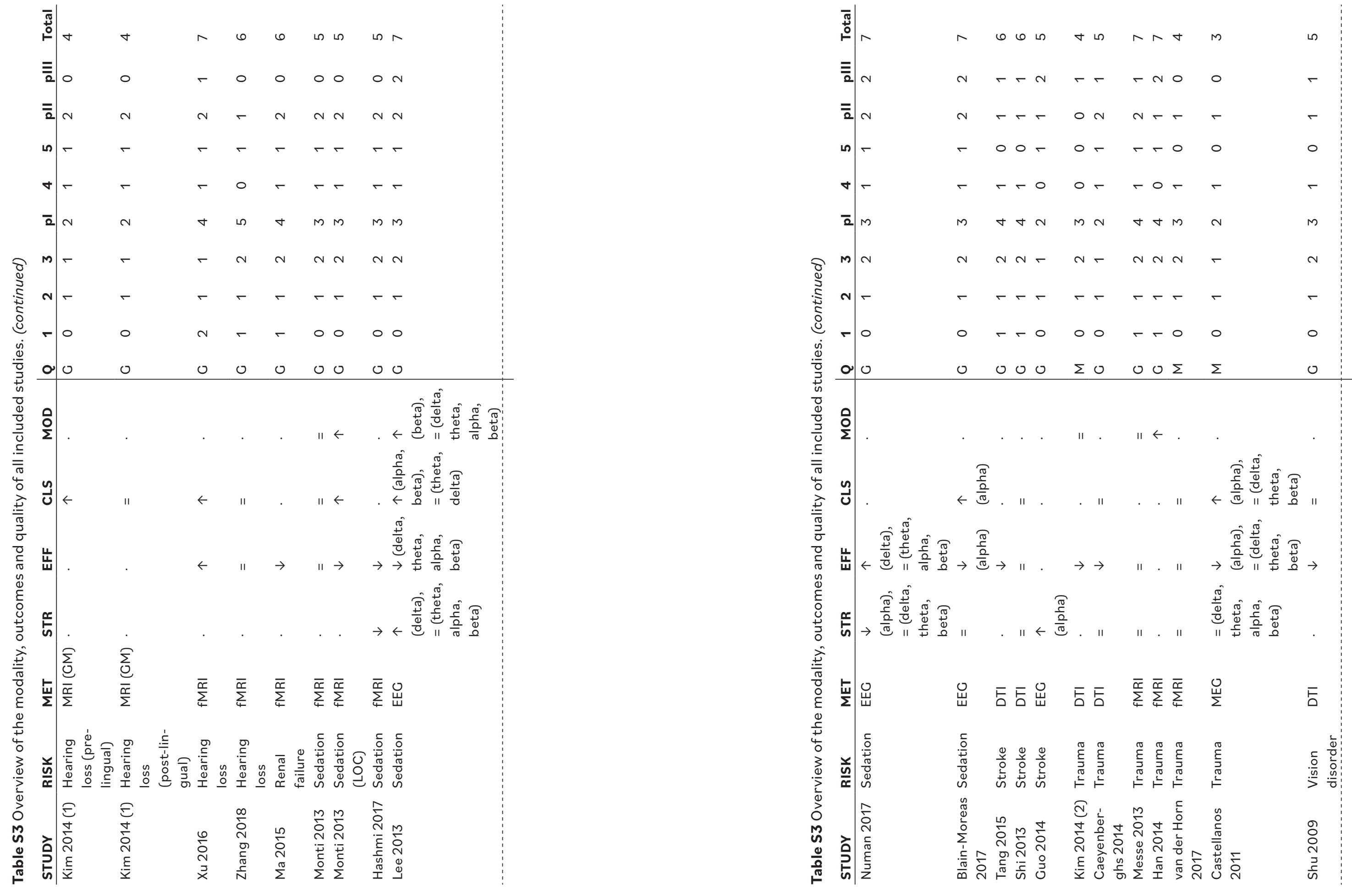


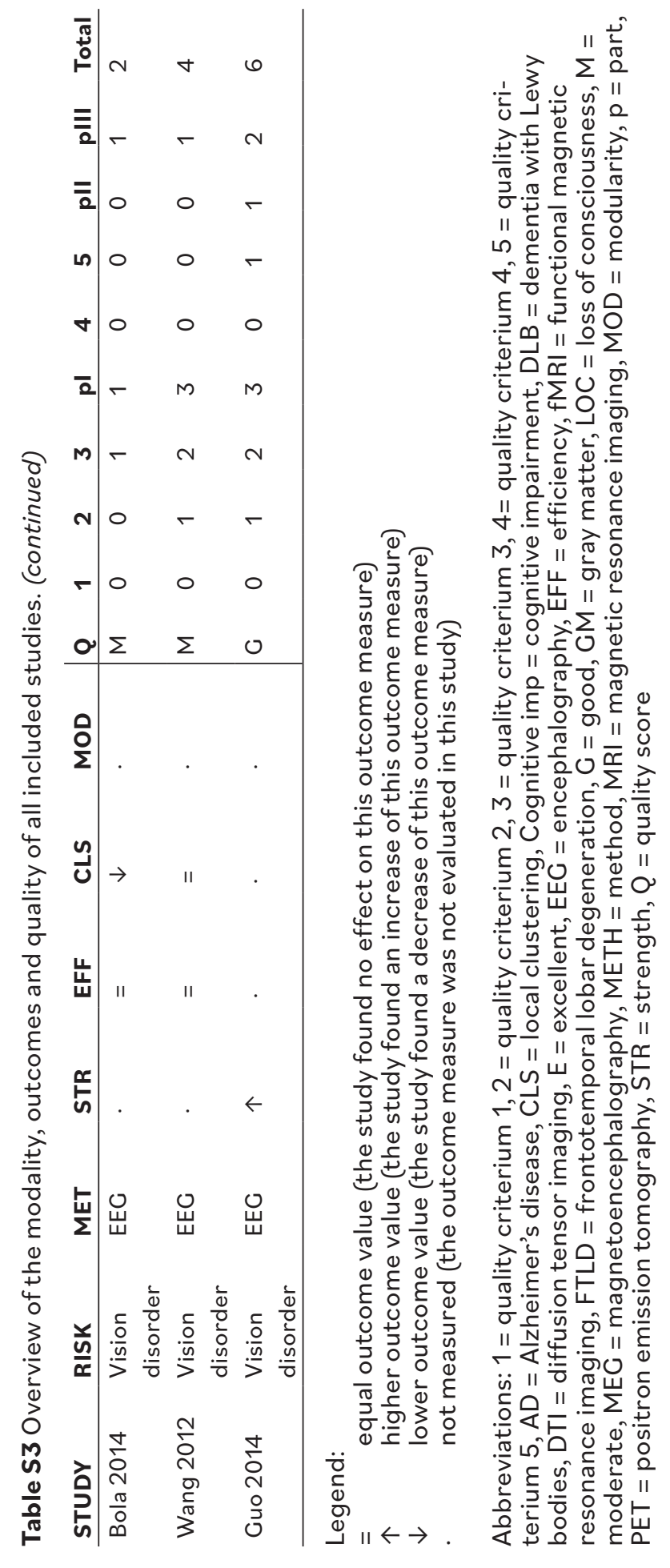




\section{Chapter 3}

Predisposition for delirium and EEG characteristics

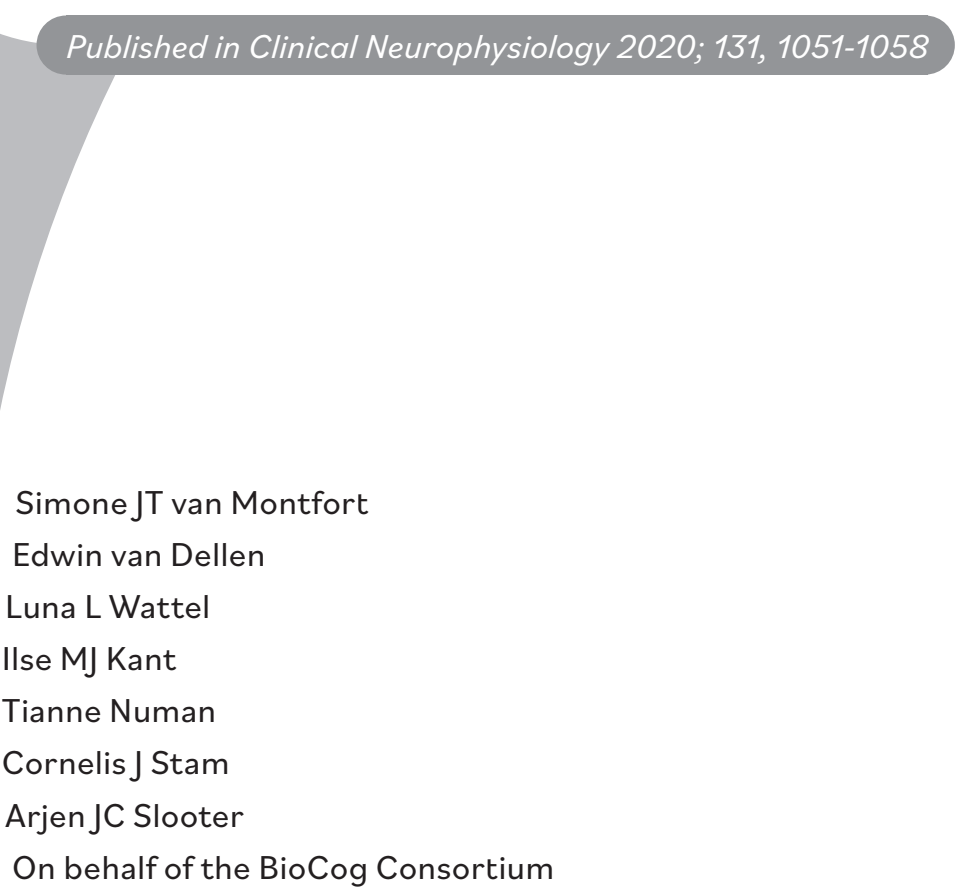




\section{Abstract}

\section{Objective}

Delirium is associated with increased electroencephalography (EEG) delta activity, decreased connectivity strength and decreased network integration. To improve our understanding of development of delirium, we studied whether non-delirious individuals with a predisposition for delirium also show these EEG abnormalities.

\section{Methods}

Elderly subjects $(\mathrm{N}=206)$ underwent resting-state EEG measurements and were assessed on predisposing delirium risk factors, i.e. older age, alcohol misuse, cognitive impairment, depression, functional impairment, history of stroke and physical status. Delirium-related EEG characteristics of interest were relative delta power, alpha connectivity strength (phase lag index) and network integration (minimum spanning tree leaf fraction). Linear regression analyses were used to investigate the relation between predisposing delirium risk factors and EEG characteristics that are associated with delirium, adjusting for confounding and multiple testing.

\section{Results}

Functional impairment was related to a decrease in connectivity strength (adjusted $\mathrm{R}^{2}=0.071, \beta=0.201, \mathrm{p}<0.05$ ). None of the other risk factors had significant influence on EEG delta power, connectivity strength or network integration.

\section{Conclusions}

Functional impairment seems to be associated with decreased alpha connectivity strength. Other predisposing risk factors for delirium had no effect on the studied EEG characteristics.

\section{Significance}

Predisposition for delirium is not consistently related to EEG characteristics that can be found during delirium.

\section{Introduction}

Delirium is an acute neuropsychiatric syndrome, predominantly characterized by a disturbance of attention and awareness with additional cognitive dysfunction ${ }^{1}$. It is a common and serious clinical complication of another medical condition, affecting over $10 \%$ of hospitalized elderly patients ${ }^{2}$. Delirium is related to poor outcomes, such as long-term cognitive impairment and death ${ }^{2}$. The development of delirium is often not the result of one factor, but rather of an interaction of various risk factors ${ }^{2-4}$. Risk factors for delirium can be distinguished into predisposing and precipitating factors ${ }^{3}$. Predisposing risk factors determine the baseline vulnerability to delirium, for example due to older age. Precipitating risk factors for delirium are acute changes that trigger the syndrome, for example sedation. A variety of risk factors have been recognized, but the underlying biological mechanisms leading to vulnerability to delirium remain poorly understood.

Delirium appears to be related to global neurophysiological disturbances, which can be measured on three different levels ${ }^{5-8}$ (Figure 1). Focusing on the frequency spectrum of the neurophysiological signal, previous studies have shown slowing of resting state electroencephalography (EEG) activity during delirium, most accurately characterized by an increase in relative delta power ${ }^{5,6}$. In addition, focusing on functional connectivity (i.e. statistical interdependencies between activity of remote brain regions which are assumed to reflect communication between these regions ${ }^{9}$ ), delirium has been characterized by decreased functional connectivity strength in the alpha frequency band ${ }^{5,6,10}$. Patterns of functional connectivity can be represented as networks, which can subsequently be analyzed with methods derived from network theory ${ }^{11,12}$. Global organizational patterns, such as network efficiency and network integration, can be calculated from these functional networks ${ }^{11-13}$. Focusing on functional network characteristics, delirium has been associated with impaired network integration 5,8,10 Therefore, it is hypothesized that delirium is a disconnection syndrome, reflecting a breakdown of functional brain networks $6,14,15$

However, it is unknown if these neurophysiological alterations coincide with the onset of delirium, or reflect vulnerability for the disorder. The aim of 
this study was to test the hypothesis that predisposing delirium risk factors induce similar neurophysiological alterations as during delirium. In this way, vulnerability may lower the threshold for a transition from a healthy state towards disturbed brain activity that manifests as delirium. More specifically, we hypothesized that predisposing risk factors for delirium are associated with increased delta power, decreased connectivity strength and decreased network integration in the alpha frequency band (Figure 1). To gain more insight in the multifactorial nature of the disorder, we additionally evaluated the cumulative effect of the predisposing risk factors on these EEG characteristics.

\section{Methods}

\section{Design and study population}

The subjects for this study derive from the Biomarker Development for Postoperative Cognitive Impairment in the Elderly (BioCog) project at the University Medical Center (UMC) Utrecht ${ }^{16}$. In this cross-sectional substudy, elderly individuals were included, consisting of non-hospitalized participants that were on the waiting list for elective surgery, recruited via the University Medical Center (UMC) Utrecht (i.e. orthopedic-, cardiac-, gastro-intestinal-, maxillofacial- or otorhinolaryngologic surgery), as well as participants that were recruited via a local general practitioner. Inclusion criteria were a European ancestry, age of 65 year or above, and a signed informed consent for the study. Participants with one or more of the following characteristics were excluded: a life expectancy shorter than a year; an indication for (early) dementia as indicated with a score of 23 or lower on the Mini Mental State Examination (MMSE) ${ }^{17}$; missing EEG data. EEG measurements and clinical assessments were performed on the same day.
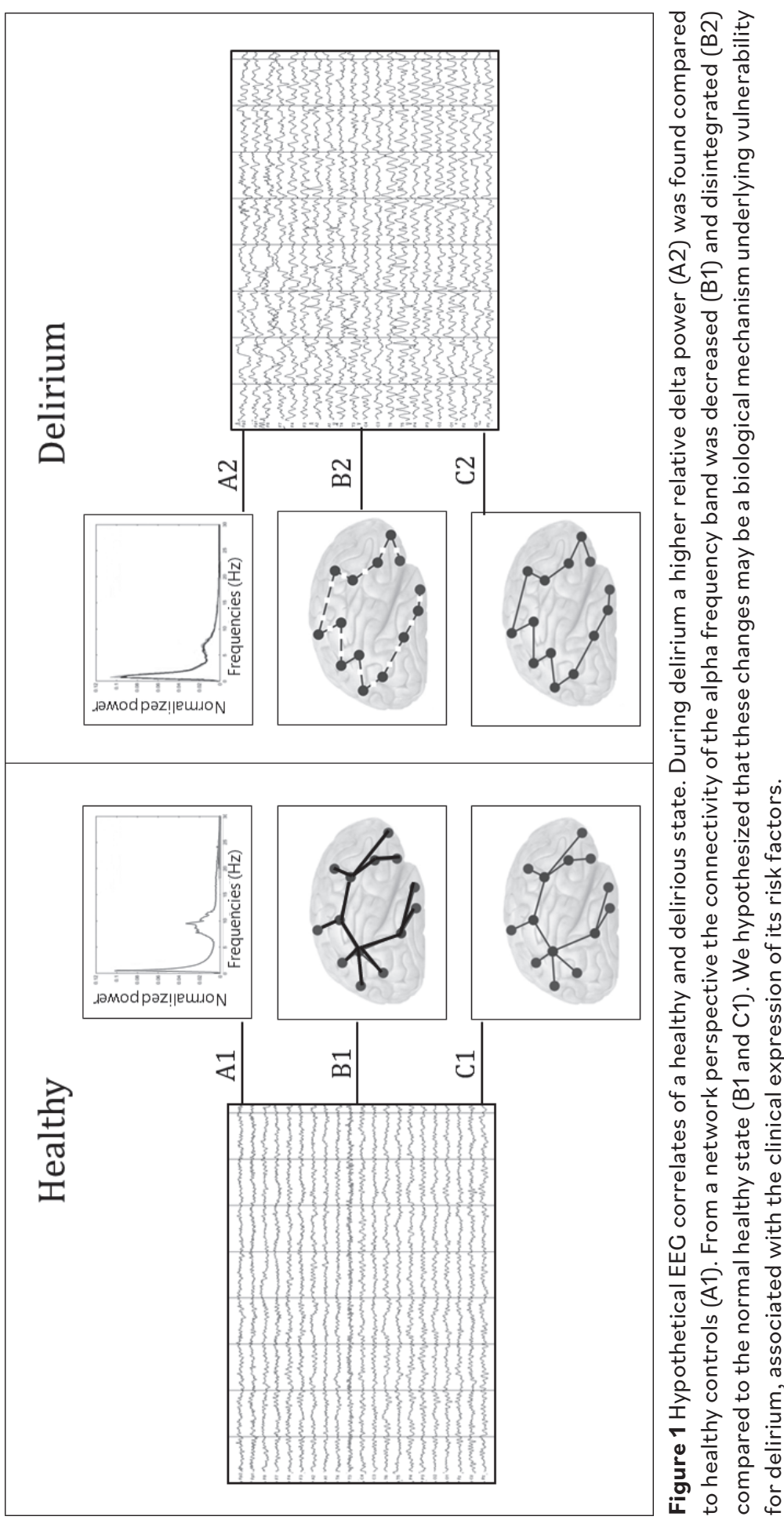


\section{Clinical assessment}

The risk factors investigated in this study were based on a high quality review ${ }^{3}$. We were not able to evaluate all risk factors described in the review, i.e. participants with dementia, hearing or visual impairment and history of delirium were not available, and comorbidity was not measured within this study.

Age and history of transient ischemic attack or stroke

To determine age and history of transient ischemic attack (TIA) or stroke, the medical records of the participants were used. If this information was not available, participants were asked whether they had experienced a TIA or stroke. If either or both were positive, this risk factor was considered present.

\section{Alcohol misuse}

The self-reported Alcohol Use Disorders Identification Test (Audit) was used to determine the risk factor of alcohol misuse. The Audit is a validated questionnaire of 10 items that assesses alcohol consumption, drinking behaviors, and alcohol-related problems ${ }^{18,19}$. The questions were scored on a 5 -point Likert scale (0-4). A cut-off value of 8 points was used to distinguish between normal and harmful alcohol consumption ${ }^{20}$.

\section{Cognitive impairment}

To determine cognitive impairment, the MMSE was used. The MMSE is a short examination that is often used in clinical practice ${ }^{17}$. The continuous outcome measure was the total score (0-30).

\section{Depression}

To estimate depression, the validated Hospital Anxiety and Depression Scale (HADS) was used ${ }^{21,22}$. For the current study, only the 7 items assessing depression were used. They were scored on a 4-point Likert scale (0-3) with a maximum score of 21 . We considered participants with a score of 7 or above as depressed ${ }^{23}$.
Functional impairment

Functional impairment was measured with the validated Barthel Index following the Hamburg classification manual ${ }^{24-26}$. The continuous outcome measure was the total score (0-100), where the maximum score of 100 indicates fully independent functional ability.

\section{Physical status}

The American Society of Anesthesiologists (ASA) validated classification is widely used for the assessment of preoperative physical status ${ }^{27,28}$, ranging from I. healthy; II. mild systematic disease; III severe systematic disease that is not incapacitating; IV. incapacitating systematic disease that is a constant threat to life; to V. moribund status, not expected to survive for 24 hours without surgery ${ }^{29}$. The used outcome measure was dichotomous, where an ASA-score of I was classified as healthy and an ASA-score of II or higher as unhealthy.

Estimated intelligence coefficient (IQ)

The validated Dutch reading test for adults 'Nederlandse leestest voor volwassenen' (NLV) was used to estimate premorbid IQ ${ }^{30}$. The participant was requested to read aloud a list of 50 words. The pronunciation of each word was scored on correctness by a trained assessor. The raw score ranges from 0-100 and was converted to an estimated IQ score using the NLV norm table ${ }^{30}$.

\section{EEG recordings, selection and preprocessing}

A 5-minute EEG recording was performed using a 32-electrode cap (Braincap MR, Brain Products $\mathrm{GmbH}$, Germany) at the positions of the 10-20 system. BrainVision Recorder (Brain Products $\mathrm{GmbH}$, Germany) was used at a sample frequency of $5000 \mathrm{~Hz}$. During recording, the participants sat upright and were awake with their eyes closed. Electrode impedance was kept below $5 \mathrm{k} \Omega$.

The quality of the EEG recordings was independently inspected visually by two researchers (ED and LW) in BrainVision Analyzer 2 software (Brain Products $\mathrm{GmbH}$, Germany). The first 10 artifact-free epochs of 8 seconds were selected for further analyses, as this was previously shown 
to be sufficient for stable results ${ }^{6,31,32}$. The first 10 artifact-free epochs of 8 seconds were selected for further analyses. Signals from electrodes TP9 and TP10 were excluded due to muscle artifacts and signals from the electrocardiography (ECG) electrode discarded from the analysis, leaving a total of 29 electrodes included in the analysis. Data was re-referenced towards an average reference that included all electrodes (except A1 and A2). The unfiltered EEG recordings were down sampled to $512 \mathrm{~Hz}$ using cubic spline interpolation.

BrainWave software was used for further analysis (v0.9.152.12.5; freely available at: http://home.kpn.nl/stam7883/). Data were band-pass filtered into five frequency bands: delta $(0.5-4 \mathrm{~Hz})$, theta $(4-8 \mathrm{~Hz})$, alpha $(8-13 \mathrm{~Hz})$, beta $(13-25 \mathrm{~Hz})$ and gamma $(25-48 \mathrm{~Hz})$

\section{EEG outcomes}

Relative delta power

For each subject, the relative spectral power was computed using a fast Fourier transformation and averaged over all channels and epochs. Relative spectral power was calculated as the ratio of the power of the corresponding band (i.e. delta, theta, alpha, beta or gamma) and the total power. As previous studies have shown, slowing of resting state EEG activity during delirium is most accurately characterized by an increase in relative delta power ${ }^{5,6}$. Therefore, we have only used the relative delta power for further analyses.

Mean phase lag index (PLI)

The mean phase lag index (PLI) was used to measure functional connectivity strength with minimal bias due to volume conduction ${ }^{33,34}$. The PLI was calculated between each channel $i$ and $j$ based on their instantaneous phase difference $\left(\Delta \phi_{\mathrm{t}}\right)$ using the following definition:

$$
P L I_{i j}=\left|\left\langle\operatorname{sign}\left(\Delta \varphi_{t}\right)\right\rangle\right|
$$

The $\operatorname{sign}(\Delta \phi t)$ is 1 for all positive phase differences and -1 for all negative phase differences, which was averaged over an epoch. The absolute value of this average is the PLI, giving a score between 0 (i.e. no phase synchronization or equal in leading and lagging over the epoch) and 1 (i.e. complete phase-locking) between each channel $i$ and $j$ in the alpha frequency band, resulting in a connectivity matrix of PLI values. As previous studies have shown an impaired connectivity strength specifically in the alpha frequency band during delirium ${ }^{5,6}$, only PLI values of the alpha frequency band were calculated.

The minimum spanning tree (MST) can be regarded as the backbone of the original network, connecting all nodes without forming loops ${ }^{35,36}$ (Figure 2). The MST of a network with $\mathrm{N}$ nodes always contains $\mathrm{N}-1$ edges, which allows a reliable comparison with another network with the same number of nodes ${ }^{32,35,36}$. PLI values of the connectivity matrix were ranked and the highest PLI value was included as the first MST connection using Kruskal's algorithm ${ }^{37}$ The second highest PLI value was then added as an MST connection, until all nodes (EEG channels) were connected. If adding a connection would result in a loop or triangle, this connection was discarded and the next PLI value was evaluated. For each participant, functional connectivity strength was calculated by averaging the alpha frequency band PLI values of included connections in the MST of all 10 epochs. For readability purposes, the term 'mean PLI' is used in the following parts of the article to indicate these mean alpha frequency band PLI values of the MST.

\section{MST leaf fraction}

The MST leaf fraction was used to measure network integration. It describes the proportion of nodes with a degree of one, i.e. nodes that are connected to only one other node ${ }^{35,36}$. A small leaf fraction means that the network has few nodes that are connected to only one other node, describing a network that is sparsely integrated (Figure 2). A large leaf fraction means that the network has many nodes that are connected to only one other node, describing a network that is highly integrated ${ }^{35,36,38}$. Since previous studies on delirium have found disruptions in the leaf fraction in the alpha frequency band specifically ${ }^{5,6,8}$, we focused on the alpha frequency band in our analyses. For each participant, network integration was calculated by averaging the MST leaf fraction values of the alpha frequency band of all 10 epochs. For readability purposes, the term 'MST leaf fraction' is used in the 
following parts of this article to indicate these alpha frequency band MST leaf fraction values.

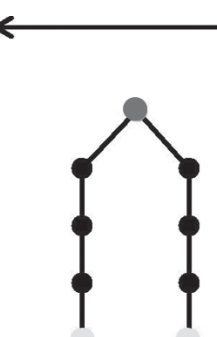

Path-like Tree

\section{Minimum Spanning Tree}

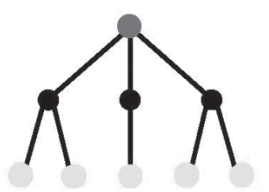

Hierarchical Tree

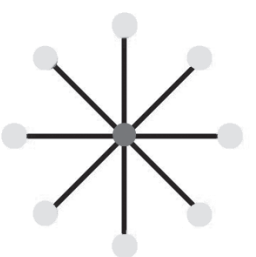

Star-like Tree
Figure 2 Schematic representation of the minimum spanning tree. Minimum spanning trees can conceptually range between a path-like tree (a sparsely integrated network) and a star-like tree (a highly integrated network). The shown path-like tree has two leaf nodes (light grey), i.e. nodes that are connected to only one other node, describing a network that is sparsely integrated. A path-like tree has a small leaf fraction. At the other end of the spectrum is a star-like tree, which has in this example one central node (dark grey) and eight leaf nodes (light grey). It thus has a high leaf fraction and information can spread easily across the network, but the central node in the star-like tree will easily be overloaded with information. A hierarchical tree is a hypothesized optimal topology, combining a relatively high efficiency, while the relatively low maximum number of connections per node prevents the overload of central hub regions.

\section{Statistical analysis}

The effect of all seven risk factors on the three outcome measures, adjusted for gender and IQ, was tested with multivariate linear regression models. The association of the individual risk factors on the three outcome measures (i.e. relative delta power, mean PLI, MST leaf fraction) was analyzed with univariate linear regression models. We performed additional, exploratory analyses on the extremes of the distribution of a possible indicator (highest versus lowest quintile) to avoid the report of false negative findings.

Multicollinearity between the different risk factors was tested with a Spearman's rank order correlation, revealing no correlations between the seven risk factors $(r<0.2)$. The mean PLI and the MST leaf fraction were not normally distributed and were log transformed for all analyses. A likelihood ratio test revealed linearity of all continuous variables. False Discovery Rate (FDR) correction was performed to control for multiple testing in the univariate and multivariate models using the Benjamini and
Hochberg method ${ }^{39,40}$. After FDR correction, a corrected $p$-value below 0.05 was considered statistically significant ${ }^{40}$. Statistical analyses were performed in IBM SPSS Statistics version 21

\section{Results}

\section{Demographics}

In this study, 230 participants were included, of whom 181 subjects were on the waiting list for elective surgery in het UMC Utrecht, and 49 were recruited via a general practitioner. From the included participants, eight were excluded due to insufficient EEG quality and 16 were excluded due to missing clinical data. Our total sample consisted of 206 participants with complete data on all clinical variables. Table 1 shows the demographics and risk factors for delirium in the sample. A mean power spectrum (Figure S1), a topographical power plot (Figure S2) and a typical MST network (Figure S3) can be found in the Supplementary Information. 
Table 1 Demographics and risk factors for delirium in the total sample.

\begin{tabular}{ll} 
& Total sample $(\mathrm{n}=206)$ \\
\hline Male, $\mathrm{n}(\%)$ & $137(67)$ \\
IQ, mean \pm SD & $104.2 \pm 12.4$ \\
Age in years, mean \pm SD & $71.3 \pm 4.8$ \\
Lowest quintile, cut-off, $\mathrm{n}(\%)$ & $\leq 67,37(18)$ \\
Highest quintile, cut-off, $\mathrm{n}(\%)$ & $\geq 76,43(17)$ \\
Alcohol misuse & \\
Yes, $\mathrm{n}(\%)$ & $10(5)$ \\
No, $\mathrm{n}(\%)$ & $196(95)$ \\
MMSE (cognitive impairment), mean \pm SD & $28.5 \pm 1.5$ \\
Lowest quintile, cut-off, $\mathrm{n}(\%)$ & $\leq 26,15(12)$ \\
Highest quintile, cut-off, $\mathrm{n}(\%)$ & $30,67(33)$ \\
HADS (depression) & \\
Yes, $\mathrm{n}(\%)$ & $20(10)$ \\
No, $\mathrm{n}(\%)$ & $186(90)$ \\
BI (functional impairment), mean \pm SD & $97.7 \pm 5.6$ \\
Lowest quintile, cut-off, $\mathrm{n}(\%)$ & $\leq 99,43(21)$ \\
Highest quintile, cut-off, $\mathrm{n}(\%)$ & $100,163(79)$ \\
History of TIA/stroke & \\
Yes, $\mathrm{n}(\%)$ & $26(13)$ \\
No, $\mathrm{n}(\%)$ & $180(87)$ \\
Physical status & \\
Healthy, $\mathrm{n}(\%)$ & $30(15)$ \\
Unhealthy, $\mathrm{n}$ (\%) & $176(85)$ \\
\hline
\end{tabular}

Abbreviations: $I Q$ = intelligence coefficient, MMSE = Mini Mental State Examination, HADS = Hospital Anxiety and Depression Scale, BI = Barthel Index, TIA = transient ischemic attack

Multivariate models

The results of the multivariate models, to test the effect of all seven risk factors on the three outcome measures (i.e. relative delta power, mean PLI, MST leaf fraction), are shown in Table 2. Functional impairment remained associated with mean PLI, independent of other risk factors, IQ or gender $\left(F(9,196)=1.671\right.$, adjusted $R^{2}=.071, \beta=.198, p=.018, p<0.05$ after FDR correction). The multivariate models for relative delta power and MST leaf fraction did not show significant effects of the other delirium risk factors.
Univariate models

The results of the univariate models on individual risk factors and the three outcome measures, are shown in Table 2. A significant effect of functional impairment on mean PLI was found $\left(F(1,204)=7.85\right.$, adjusted $R^{2}=.032$, $\beta=.193, p=.006, p<0.05$ after FDR correction) (Figure 3 ). None of the other delirium risk factors were associated with any of the outcome measures. Notably, after FDR correction, age was not significantly associated with mean PLI $\left(F(1,204)=3.859\right.$, adjusted $\left.R^{2}=.014, \beta=-.136, p=.051\right)$ and MST leaf fraction $\left(F(1,204)=3.125\right.$, adjusted $\left.R^{2}=.010, \beta=-.123, p=.079\right)$.

Post-hoc extreme quintiles comparisons

Comparing the extreme quintiles within the continuous variables (i.e. age, cognitive impairment and functional impairment), showed a significant difference of the highest quintile of functional impairment compared to the lowest quintile on mean PLI ( $\mathrm{t}(106)=-3.502, \mathrm{p}=0.001)$ ) and on MST leaf fraction $(t(99)=-2.690, p=0.008)$ ) (Table S1). Other comparisons within the extreme quintiles of the continuous variables did not show significant differences. 

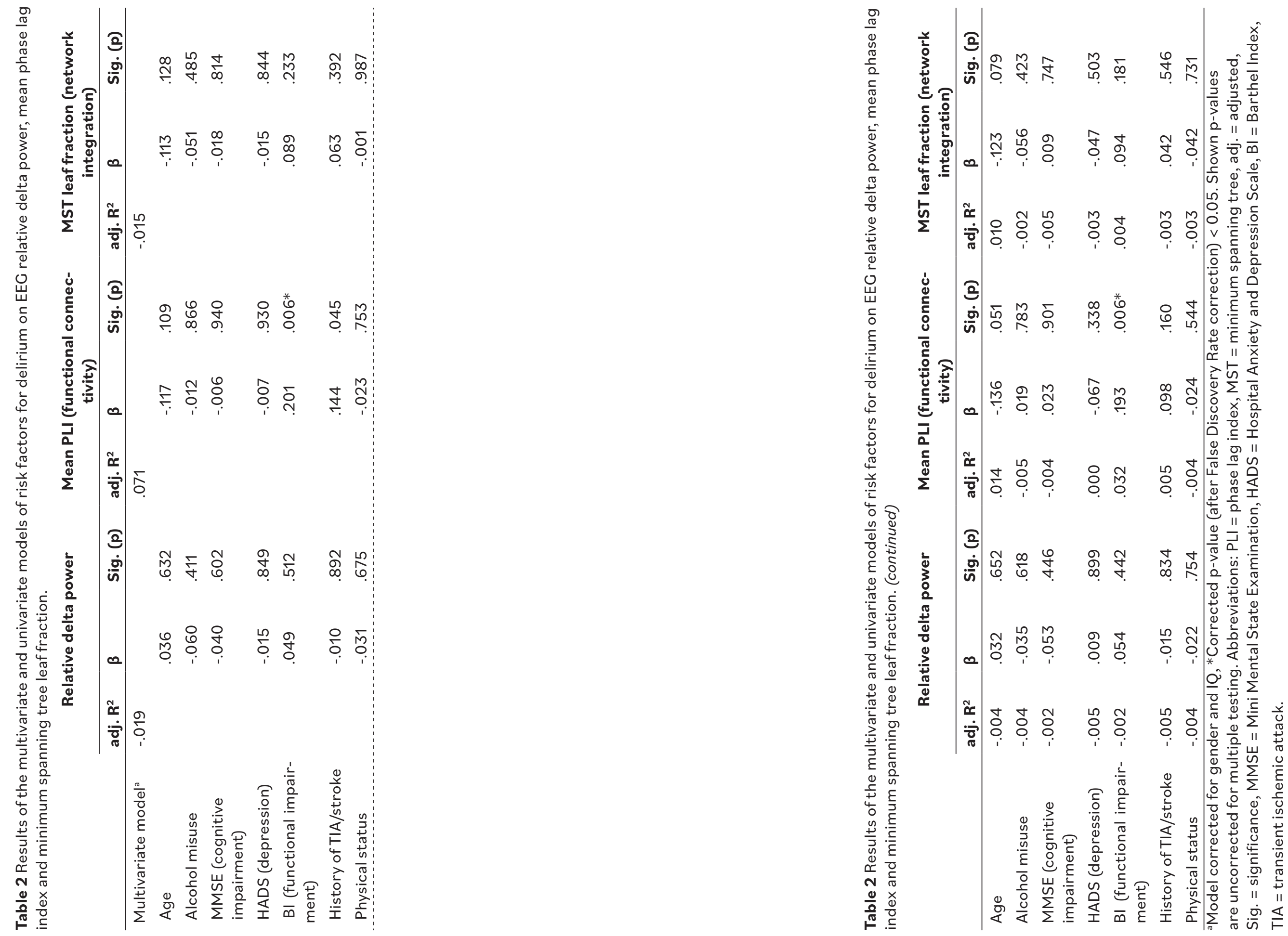


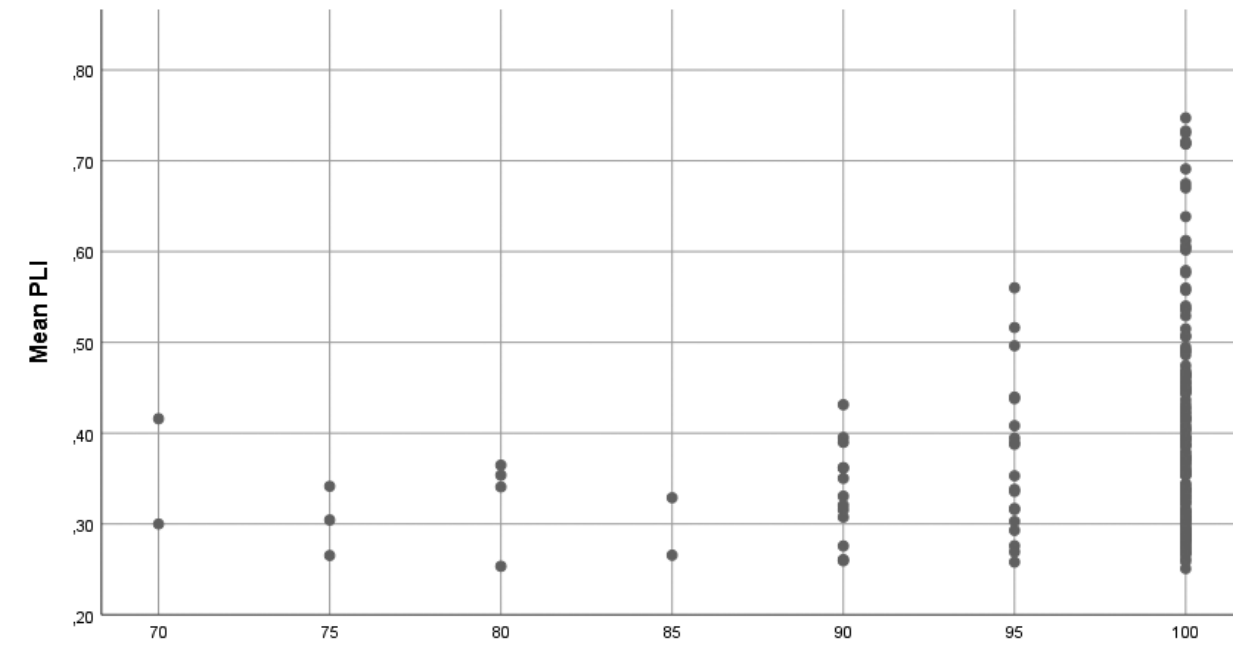

Barthel Index

Figure 3 The relationship between functional impairment and EEG connectivity strength. A significant association was found between functional impairment, as measured with the Barthel Index, and EEG connectivity strength, as measured with the mean phase lag index of the minimum spanning tree in the alpha frequency band. The association was independent of other risk factors for delirium, IQ or gender. Abbreviations: $\mathrm{PLI}=$ phase lag index.

\section{Discussion}

In summary, functional impairment in non-delirious individuals was related to a decrease in EEG connectivity strength, but was not related to relative delta power or measures of network integration. A model that combined the seven studied risk factors showed that this association was independent from the other predisposing risk factors, gender or IQ. No individual or cumulative relation was found between the other risk factors (i.e. age, alcohol misuse, cognitive impairment, depression, history of TIA or stroke, physical status) and relative delta power, connectivity strength or measures of network integration. These findings suggest that most predisposing risk factors for delirium do not affect EEG characteristics that are disturbed during delirium, with exception of the risk factor functional impairment. Therefore, predisposition for delirium is not consistently related to alterations in EEG characteristics.

Associations between predisposing risk factors for delirium and EEC characteristics were investigated in different ways, i.e. in separate regression models, in combined regression models, and in extreme quintiles comparisons, revealing a robust finding of the relationship between functional impairment and connectivity strength. Although a relationship has been found between physical health and neurophysiological outcomes ${ }^{41}$, our study is the first to investigate the relationship between functional impairment and neurophysiological alterations in a cohort of non-delirious elderly at risk for delirium. The measurement of functional impairment may provide a reflection of physical frailty in our sample. Physical frailty is defined as an age-related syndrome of decreased reserve causing vulnerability to physiological stressors and could manifest as functional dependency ${ }^{42,43}$. Previous studies have shown that frailty is strongly associated with the risk of delirium ${ }^{44-46}$. However, as the risk factors included in this study were based on a landmark paper that did not identify frailty as a risk factor for delirium ${ }^{3}$, we did not consider frailty as a separate risk factor. The clinical overlap between frailty and functional impairment may explain why only functionally impaired individuals showed a similarly impaired functional connectivity as previously found during delirium ${ }^{5,6}$, while individuals with other risk factors for delirium did not. However, we found relatively low adjusted $\mathrm{R} 2$ and beta values for the association of functional impairment and decreased connectivity strength (adjusted $R 2=0.071, \beta=0.201$ ). Although this relationship was statistically significant, the explanatory power was weak as only $7.1 \%$ of the variation in connectivity strength could be explained by functional impairment. Future research is needed to clarify the exact relationship between frailty, functional impairment and delirium, and the similarity in their underlying biological mechanisms.

The other risk factors for delirium were not related to the studied EEC alterations, which is not compatible with previous studies indicating that age and cognitive impairment were associated with decreased functional connectivity ${ }^{47-54}$. Interestingly, although non-significant, age did show a similar pattern in the univariate model on functional connectivity and cognitive impairment did show a similar pattern comparing the extreme quintiles on functional connectivity, as compared to previous studies ${ }^{47-54}$ Whereas previous studies were mostly performed using a case-control design comparing clinically diagnosed patients to healthy controls ${ }^{47-54}$, we evaluated multiple risk factors for delirium in a group of elderly individuals. 
These methodological differences may impede a direct comparison of our study to the previous literature.

Our findings suggest that predisposing delirium risk factors do not consistently impact EEG characteristics that are disturbed during delirium. Delirium is a state with an acute onset ${ }^{1}$. The related neurophysiological changes may only occur during delirium, or as a result of precipitating factors in the days before clinical manifestation of the syndrome ${ }^{55,56}$. It could be that predisposing risk factors still impair the structural network (i.e. lead to decreased anatomical as opposed to functional connections) ${ }^{57}$, while precipitating risk factors may influence the functional network ${ }^{5,58-61}$. In severe cases, the structural network may subsequently alter the functiona network, as structural and functional networks are robustly linked ${ }^{62-65}$ This alternative hypothesis is supported by the finding that predisposing risk factors were found to be associated with decreased structura connectivity as well as efficiency ${ }^{10,57}$. Future research evaluating the effects of predisposing risk factors on structural brain network characteristics will provide more insight in the validity of the network theoretical framework for understanding the vulnerability to delirium.

This study is the first to empirically investigate the association between predisposing risk factors for delirium and EEG characteristics in the same study population. It represents a novel approach to unravel the pathophysiological mechanisms of a very common medical condition. Biaslimiting EEG measures on a substantial amount of data, i.e. 80 seconds of EEG recording per participant, were used ${ }^{32}$. Nevertheless, a limitation of the current study is the selection of the participant sample. Since the participants derived from a relatively healthy population, this resulted in low variability and low frequency of some of the (dichotomous) risk factors. Furthermore, the measures for functional impairment and physical status, may not have been sufficiently sensitive. The Barthel Index shows a ceiling effect, and the ASA-score is only a very rough indicator for physical status 28,67 . Although the landmark review on delirium risk factors that we used did include the most prominent and robust factors, it did not include al known risk factors. Another possible limitation of the study might be that we could not detect associations between different delirium risk factors.
In our study risk factors for delirium were not significantly related to each other. However, due to the limited clinical measurements used in the study, we cannot draw strong conclusions on (lack of) associations among different risk factors for delirium. A significant limitation of the study is that we had no information on medication use of the participants during the EEG measurement. Although the participants were derived from a relatively healthy population and were not hospitalized, we cannot preclude effects of possible medication on the EEG signal. Furthermore, as our aim was to test the hypothesis that predisposing delirium risk factors induce similar neurophysiological alterations as during delirium, we did not evaluated neurophysiological outcomes that are not known as being altered during delirium. Impairments may have shifted to other frequency bands or outcomes in patients at risk for delirium. A more data-driven approach may be used in future studies to learn more about other possible (neurophysiological) alterations in persons at risk of delirium. In addition, as the spatial resolution of EEG is low, we were not able to include anatomical information, which may be of relevance for developing delirium. It would therefore be interesting to replicate our study design with functional magnetic resonance imaging ( $\mathrm{FMRI}$ ).

\section{Conclusion}

Although functional impairment was related to a decrease in connectivity strength, other predisposing delirium risk factors were not found to be associated with EEG characteristics of delirium. Therefore, vulnerability for delirium is not consistently associated with alterations in EEG characteristics, and the onset of delirium may reflect new neurophysiological alterations. 


\section{Acknowledgements}

This work was supported by funding from the European Union Seventh Framework Program under Grant Agreement No. 602461 [FP7/20072013] and funding from the Dutch Organization for Health Research and Development (ZonMW) under Grant Agreement No. 60-63600-98-711. EEC hardware and analysis software was provided by the BioCog Consortium partner Pharmaimage Biomarker Solutions $\mathrm{GmbH}$, Berlin Germany (www. pi-pharmaimage.com).

\section{References}

1. American Psychiatric Association. Diagnostic and Statistical Manual of Mental Disorders (5th Ed.). Washington, DC; 2013.

2. Marcantonio ER. Delirium in Hospitalized Older Adults. Solomon CG, ed. N EnglJ Med. 2017;377(15):1456-1466. doi:10.1056/NEJMcp1605501

3. Inouye SK, Westendorp RG, Saczynski JS. Delirium in elderly people. Lancet 2014;383(9920):911-922. doi:10.1016/S0140-6736(13)60688-1

4. Zaal IJ, Devlin JW, Peelen LM, Slooter AJC. A Systematic Review of Risk Factors for Delirium in the ICU*. Crit Care Med. 2015;43(1):40-47. doi:10.1097/ CCM.0000000000000625

5. Numan T, Slooter AJC, van der Kooi AW, et al. Functional connectivity and network analysis during hypoactive delirium and recovery from anesthesia. Clin Neurophysiol. 2017;128(6):914-924. doi:10.1016/j.clinph.2017.02.022

6. van Dellen E, van der Kooi AW, Numan T, et al. Decreased Functional Connectivity and Disturbed Directionality of Information Flow in the Electroencephalography of Intensive Care Unit Patients with Delirium after Cardiac Surgery. Anesthesiology. 2014;121(2):328-335. doi:10.1097/ALN.0000000000000329

7. Brandt SA, Kraft A, Traenkner S, Schreiber SJ, Fleischmann R, Schmidt S. Delirium is associated with frequency band specific dysconnectivity in intrinsic connectivity networks: preliminary evidence from a large retrospective pilot case-control study. Pilot Feasibility Stud. 2019;5(1):1-13. doi:10.1186/s40814-018-0388-z

8. van Montfort SJT, van Dellen E, van den Bosch AMR, et al. Resting-state fMRI reveals network disintegration during delirium. Neurolmage Clin. 2018;20:35-41. doi:10.1016/J. NICL.2018.06.024

9. Aertsen AM, Gerstein GL, Habib MK, Palm G. Dynamics of neuronal firing correlation modulation of "effective connectivity."J Neurophysiol. 1989;61(5):900-917. doi:10.1152/ jn.1989.61.5.900

10. van Montfort SJT, van Dellen E, Stam CJ, et al. Brain network disintegration as a final common pathway for delirium: a systematic review and qualitative meta-analysis. Neurolmage Clin. 2019;23. doi:10.1016/j.nicl.2019.101809

11. van Straaten ECW, Stam CJ. Structure out of chaos: Functional brain network analysis with EEG, MEG, and functional MRI. Eur Neuropsychopharmacol. 2013;23(1):7-18. doi:10.1016/j.euroneuro.2012.10.010

12. Stam CJ, van Straaten ECW. The organization of physiological brain networks. Clin Neurophysiol. 2012. doi:10.1016/j.clinph.2012.01.011 
13. Bullmore E, Sporns O. Complex brain networks: Graph theoretical analysis of structural and functional systems. Nat Rev Neurosci. 2009;10(3):186-198. doi:10.1038/nrn2575

14. Sanders RD. Hypothesis for the pathophysiology of delirium: Role of baseline brain network connectivity and changes in inhibitory tone. Med Hypotheses. 2011;77(1):140 143. doi:10.1016/j.mehy.2011.03.048

15. Young JWS. The network model of delirium. Med Hypotheses. 2017;104:80-85 doi:10.1016/j.mehy.2017.05.027

16. Winterer $\mathrm{G}$, Androsova $\mathrm{G}$, Bender $\mathrm{O}$, et al. Personalized risk prediction of postoperative cognitive impairment - rationale for the EU-funded BioCog project. Eur Psychiatry 2018. doi:10.1016/j.eurpsy.2017.10.004

17. Folstein M, Anthony JC, Parhad I, Duffy B, Gruenberg EM. The meaning of cognitive impairment in the elderly. J Am Geriatr Soc. 1985;33(4):228-235. http://www.ncbi. nIm.nih.gov/pubmed/3989183. Accessed January 28, 2019.

18. Bohn MJ, Babor TF, Kranzler HR. The Alcohol Use Disorders Identification Test (AUDIT) validation of a screening instrument for use in medical settings. J Stud Alcohol, 1995;56(4):423-432. doi:10.15288/jsa.1995.56.423

19. Reinert DF, Allen JP. The Alcohol Use Disorders Identification Test: An Update of Research Findings. Alcohol Clin Exp Res. 2007;31(2):185-199. doi:10.1111/j.15300277.2006.00295.x

20. Babor T, Higgins-Biddle J, Saunders J. AUDIT: the alcohol use disorders identification test: guidelines for use in primary health care. 2001. http://apps.who.int/iris/ bitstream/handle/10665/67205/WHO_MSD_?sequence=1. Accessed January 28, 2019.

21. Zigmond AS, Snaith RP. The Hospital Anxiety and Depression Scale. Acta Psychiatr Scand. 1983;67(6):361-370. doi:10.1111/j.1600-0447.1983.tb09716.x

22. Bjelland I, Dahl AA, Haug TT, Neckelmann D. The validity of the Hospital Anxiety and Depression Scale: An updated literature review.J Psychosom Res. 2002;52(2):69-77. doi:10.1016/S0022-3999(01)00296-3

23. Vodermaier A, Millman RD. Accuracy of the Hospital Anxiety and Depression Scale as a screening tool in cancer patients: a systematic review and meta-analysis. Support Care Cancer. 2011;19(12):1899-1908. doi:10.1007/s00520-011-1251-4

24. Mahoney FI, Barthel DW. FUNCTIONAL EVALUATION: THE BARTHEL INDEX. Md State Med J. 1965;14:61-65. http://www.ncbi.nlm.nih.gov/pubmed/14258950. Accessed January 28, 2019.

25. Collin C, Wade DT, Davies S, Horne V. The Barthel ADL Index: a reliability study. Int Disabil Stud. 1988;10(2):61-63. http://www.ncbi.nlm.nih.gov/pubmed/3403500. Accessed January 28, 2019.
26. Lübke N, Meinck M, Von Renteln-Kruse W. [The Barthel Index in geriatrics. A context analysis for the Hamburg Classification Manual]. Z Gerontol Geriatr. 2004;37(4):316326. doi:10.1007/s00391-004-0233-2

27. Aronson WL, McAuliffe MS, Miller K. Variability in the American Society of Anesthesiologists Physical Status Classification Scale. AANA J. 2003;71(4):265-274. http://www.ncbi.nlm.nih.gov/pubmed/13677221. Accessed January 28, 2019.

28. Sankar A, Johnson SR, Beattie WS, Tait G, Wijeysundera DN. Reliability of the American Society of Anesthesiologists physical status scale in clinical practice. Br J Anaesth. 2014;113(3):424-432. doi:10.1093/bja/aeu100

29. Owens WD, Felts JA, Spitznagel EL. ASA physical status classifications: a study of consistency of ratings. Anesthesiology. 1978;49(4):239-243. http://www.ncbi.nlm. nih.gov/pubmed/697077. Accessed January 28, 2019.

30. Mulder, J., Bouma, J. M. \& Schmand B. Handboek Neuropsychologische Diagnostiek. Amsterdam: Pearson Assessment and Information B.V; 2012

31. Fraschini M, Demuru M, Crobe A, Marrosu F, Stam CJ, Hillebrand A. The effect of epoch length on estimated EEG functional connectivity and brain network organisation. $J$ Neural Eng. 2016;13(3):036015. doi:10.1088/1741-2560/13/3/036015

32. van Diessen E, Numan T, van Dellen E, et al. Opportunities and methodological challenges in EEG and MEG resting state functional brain network research. Clin Neurophysiol. 2015;126(8):1468-1481. doi:10.1016/j.clinph.2014.11.018

33. Boersma M, Smit DJA, Boomsma DI, De Geus EJC, Delemarre-van de Waal HA, Stam CJ Growing Trees in Child Brains: Graph Theoretical Analysis of ElectroencephalographyDerived Minimum Spanning Tree in 5- and 7-Year-Old Children Reflects Brain Maturation. Brain Connect. 2013;3(1):50-60. doi:10.1089/brain.2012.0106

34. van Dellen E, de Waal H, van der Flier WM, et al. Loss of EEGNetwork Efficiency Is Related to Cognitive Impairment in Dementia With Lewy Bodies. Mov Disord. 2015;30(13):1785-1793. doi:10.1002/mds.26309

35. Tewarie P, van Dellen E, Hillebrand A, Stam CJ. The minimum spanning tree: An unbiased method for brain network analysis. Neuroimage. 2015;104:177-188. doi:10.1016/j. neuroimage.2014.10.015

36. Stam CJ, Tewarie P, Van Dellen E, van Straaten ECW, Hillebrand A, Van Mieghem $P$. The trees and the forest: Characterization of complex brain networks with minimum spanning trees. Int J Psychophysiol. 2014;92(3):129-138. doi:10.1016/j. ijpsycho.2014.04.001

37. Kruskal JB. On the Shortest Spanning Subtree of a Graph and the Traveling Salesman Problem. Proc Am Math Soc. 1956;7(1):48-50. https://www.jstor.org/stable/ pdf/2033241.pdf. Accessed March 20, 2019 
38. van Dellen E, Bohlken MM, Draaisma L, et al. Structural Brain Network Disturbances in the Psychosis Spectrum. Schizophr Bull. 2016;42(3):782-789. doi:10.1093/schbul/ sbv178

39. Genovese CR, Lazar NA, Nichols T. Thresholding of Statistical Maps in Functional Neuroimaging Using the False Discovery Rate. Neuroimage. 2002;15(4):870-878. doi:10.1006/NIMG.2001.1037

40. Benjamini Y, Hochberg Y. Controlling the False Discovery Rate: A Practical and Powerful Approach to Multiple Testing. J R Stat Soc Ser B. 1995;57(1):289-300. doi:10.1111/j.2517-6161.1995.tb02031.x

41. Douw L, Nieboer D, van Dijk BW, Stam CJ, TwiskJWR. A Healthy Brain in a Healthy Body: Brain Network Correlates of Physical and Mental Fitness. Lambiotte R, ed. PLoS One. 2014;9(2):e88202. doi:10.1371/journal.pone.0088202

42. Clegg A, Young J, lliffe S, Rikkert MO, Rockwood K. Frailty in elderly people. Lancet. 2013;381(9868):752-762. doi:10.1016/S0140-6736(12)62167-9

43. Fried LP, Tangen CM, Walston J, et al. Frailty in Older Adults: Evidence for a Phenotype. Journals Gerontol Ser A Biol Sci Med Sci. 2001;56(3):M146-M157. doi:10.1093/ gerona/56.3.M146

44. Pitkala KH, Laurila J V., Strandberg TE, Tilvis RS. Prognostic Significance of Delirium in Frail Older People. Dement Geriatr Cogn Disord. 2005;19(2-3):158-163. doi:10.1159/000082888

45. Eeles EMP, White S V., O'Mahony SM, Bayer AJ, Hubbard RE. The impact of frailty and delirium on mortality in older inpatients. Age Ageing. 2012;41(3):412-416. doi:10.1093/ ageing/afs 021

46. Brown CH, Max L, LaFlam A, et al. The Association Between Preoperative Frailty and Postoperative Delirium After Cardiac Surgery. Anesth Analg. 2016;123(2):430-435. doi:10.1213/ANE.0000000000001271

47. Ferreira LK, Regina ACB, Kovacevic N, et al. Aging effects on whole-brain functional connectivity in adults free of cognitive and psychiatric disorders. Cereb Cortex. 2016;26(9):3851-3865. doi:10.1093/cercor/bhv190

48. Vysata O, KukalJ, Prochazka A, Pazdera L, SimkoJ, Valis M. Age-related changes in EEC coherence. Neurol Neurochir Pol. 2014;48(1). doi:10.1016/j.pjnns.2013.09.001

49. Smit DJA, de Geus EJC, Boersma M, Boomsma DI, Stam CJ. Life-Span Development of Brain Network Integration Assessed with Phase Lag Index Connectivity and Minimum Spanning Tree Graphs. Brain Connect. 2016;6(4):312-325. doi:10.1089/ brain.2015.0359
50. Geerligs L, Rubinov M, Cam-Can, Henson RN. State and Trait Components of Functional Connectivity: Individual Differences Vary with Mental State. J Neurosci. 2015;35(41):13949-13961. doi:10.1523/JNEUROSCI.1324-15.2015

51. Minati L, Chan D, Mastropasqua $\mathrm{C}$, et al. Widespread alterations in functional brain network architecture in amnestic mild cognitive impairment. J Alzheimers Dis. 2014;40(1):213-220. doi:10.3233/jad-131766

52. Chang T-Y, Huang K-L, Ho M-Y, et al. Graph theoretical analysis of functional networks and its relationship to cognitive decline in patients with carotid stenosis.J Cereb Blood Flow Metab. 2016;36(4):808-818. doi:10.1177/0271678X15608390

53. Zeng K, Wang Y, Ouyang G, Bian Z, Wang L, Li X. Complex network analysis of resting state EEG in amnestic mild cognitive impairment patients with type 2 diabetes. Front Comput Neurosci. 2015;9:133. doi:10.3389/fncom.2015.00133

54. Frantzidis CA, Vivas AB, Tsolaki A, Klados MA, Tsolaki M, Bamidis PD. Functional disorganization of small-world brain networks in mild Alzheimer's disease and amnestic Mild cognitive impairment: An EEG study using Relative Wavelet Entropy (RWE). Front Aging Neurosci. 2014;6:224. doi:10.3389/fnagi.2014.00224

55. Evans JL, Nadler JW, Preud'homme XA, et al. Pilot prospective study of postsurgery sleep and EEG predictors of post-operative delirium. Clin Neurophysiol. 2017;128(8):1421-1425. doi:10.1016/J.CLINPH.2017.05.004

56. Numan T, van den Boogaard M, Kamper AM, Rood PJT, Peelen LM, Slooter AJC. Recognition of Delirium in Postoperative Elderly Patients: A Multicenter Study. J Am Geriatr Soc. 2017;65(9):1932-1938. doi:10.1111/jgs.14933

57. Kyeong S, Shin JE, Yang KH, Lee WS, Chung T-S, Kim J-J. Neural predisposing factors of postoperative delirium in elderly patients with femoral neck fracture. Sci Rep. 2018;8(1):7602. doi:10.1038/s41598-018-26030-2

58. Mashour, GA, Hudetz A. Neural Correlates of Unconsciousness in Large-Scale Brain Networks. Trends Neurosci. 2018;41(3):150-160.

59. Blain-Moraes S, Tarnal V, Vanini G, et al. Network Efficiency and Posterior Alpha Patterns Are Markers of Recovery from General Anesthesia: A High-Density Electroencephalography Study in Healthy Volunteers. Front Hum Neurosci. 2017;11:328. doi:10.3389/fnhum.2017.00328

60. Lee H, Mashour GA, Noh G-J, Kim S, Lee U. Reconfiguration of network hub structure after propofol-induced unconsciousness. Anesthesiology. 2013;119(6):1347-1359. doi:10.1097/ALN.0b013e3182a8ec8c

61. Maestú F, Boccaletti S, Castellanos NP, et al. Principles of recovery from traumatic brain injury: Reorganization of functional networks. Neuroimage. 2010;55(3):11891199. doi:10.1016/j.neuroimage.2010.12.046 
62. Honey $\mathrm{CJ}$, Sporns $\mathrm{O}$, Cammoun L, et al. Predicting human resting-state functional connectivity from structural connectivity. Proc Nat/ Acad Sci U S A. 2009;106(6):2035 2040. doi:10.1073/pnas.0811168106

63. Cabral J, Kringelbach M, Deco G. Functional Graph Alterations in Schizophrenia: A Result from a Global Anatomic Decoupling? Pharmacopsychiatry. 2012;45(S 01):S57S64. doi:10.1055/s-0032-1309001

64. Cabral J, Hugues E, Kringelbach ML, Deco G. Modeling the outcome of structural disconnection on resting-state functional connectivity. Neuroimage. 2012;62(3):1342 1353. doi:10.1016/j.neuroimage.2012.06.007

65. Mišić B, Betzel RF, Nematzadeh A, et al. Cooperative and Competitive Spreading Dynamics on the Human Connectome. Neuron. 2015;86(6):1518-1529. doi:10.1016/j. neuron.2015.05.035

66. van Montfort, SJT; van Dellen, E; Stam C, Ahmad A, Mentink L, Kraan C, Zalesky A, Slooter A. Brain network disintegration as a final common pathway for delirium: a systematic review. 2019.

67. Sarker S-J, Rudd AG, Douiri A, Wolfe CDA. Comparison of 2 extended activities of daily living scales with the Barthel Index and predictors of their outcomes: cohort study within the South London Stroke Register (SLSR). Stroke. 2012;43(5):1362-1369. doi:10.1161/STROKEAHA.111.645234

\section{Supplementary Information}

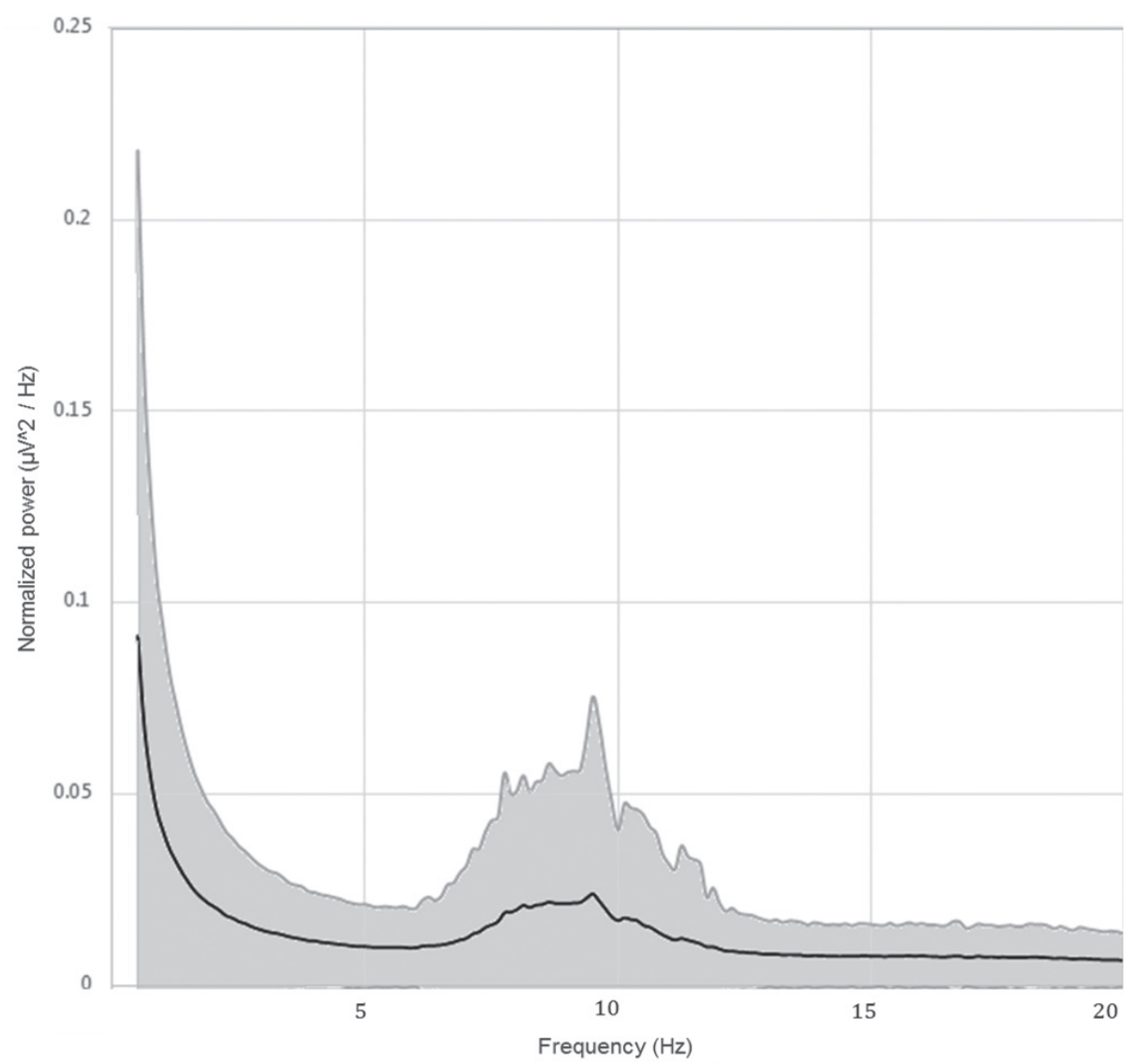

Figure S1: Mean power spectrum based on the EEG data of all participants $(N=206)$. The mean is depicted by the black line, +1 and -1 standard deviation are visualized by the grey shading. 
A
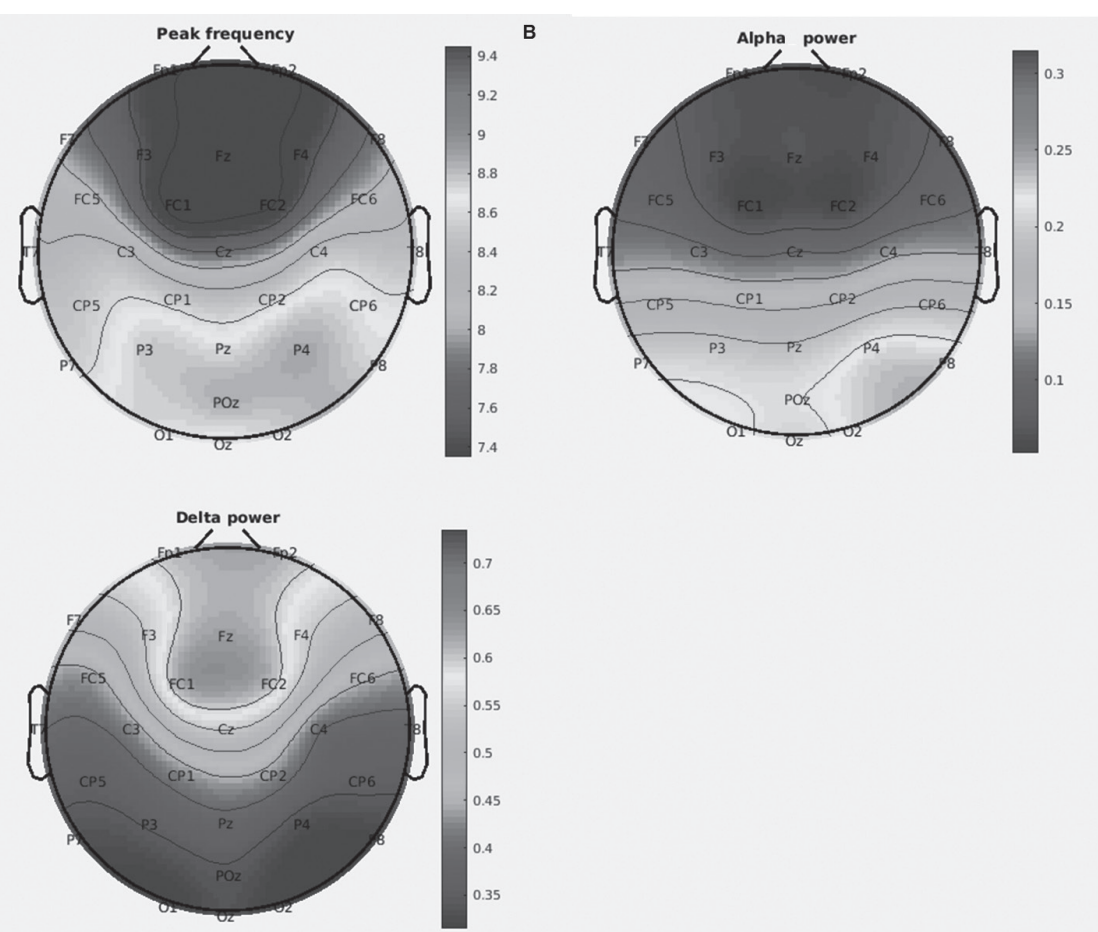

Figure S2: Topographical power plots of (A) the peak frequency, (B) the relative alpha power and (C) the relative delta power.

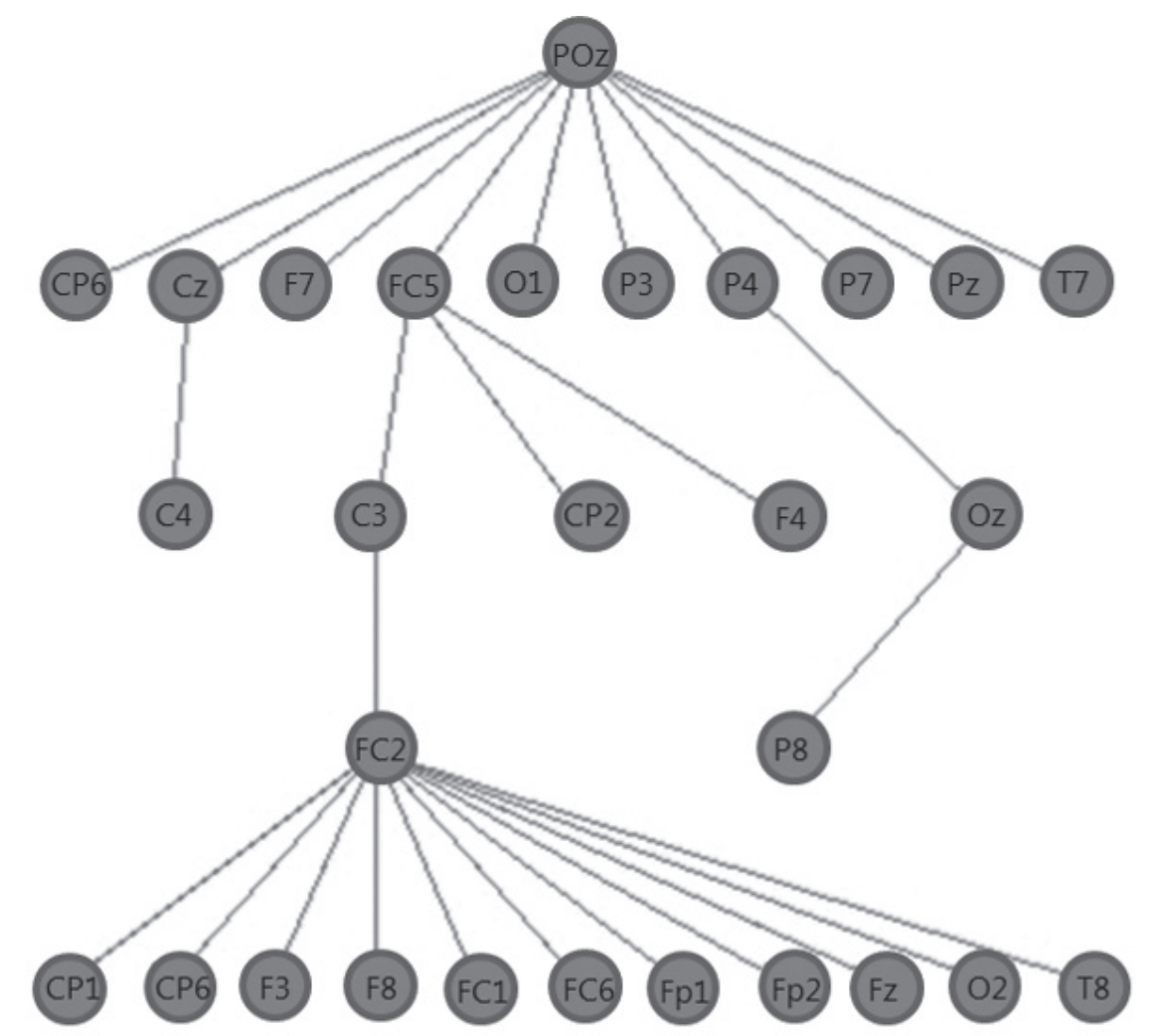

Figure S3: A minimum spanning tree of the alpha frequency band based on the EEG data of a representative subject. 
Table S1: Results of the extreme quintile comparisons t-test of relative delta power (A), mean PLI (B) and MST leaf fraction (C) in age, MMSE and Barthel Index. Abbreviations: $\mathrm{MMSE}=$ Mini Mental State Examination, $\mathrm{BI}=$ Barthel Index, $\mathrm{df}=$ degrees of freedom.

A. Relative delta power

\begin{tabular}{llll} 
& $\mathbf{d f}$ & $\mathbf{t}$ & $\mathbf{p}$ \\
\hline Age & 78 & 0.648 & 0.519 \\
MMSE (cognitive impairment) & 89 & -1.972 & 0.056 \\
BI (functional impairment) & 204 & -1.521 & 0.130 \\
B. Mean PLI & & & \\
& $\mathbf{d f}$ & $\mathbf{t}$ & $\mathbf{p}$ \\
\hline Age & 78 & -1.520 & 0.132 \\
MMSE (cognitive impairment) & 69.5 & -1.885 & 0.064 \\
BI (functional impairment) & 106.5 & -3.502 & 0.001 \\
C. MST leaffraction & & & \\
& & & $\mathbf{p}$ \\
\hline Age & $\mathbf{d f}$ & $\mathbf{t}$ & 0.081 \\
MMSE (cognitive impairment) & 78 & -1.770 & 0.056 \\
BI (functional impairment) & 89 & 1.972 & 0.008
\end{tabular}




\section{Chapter 4}

fMRI network correlates of predisposing risk factors for delirium: a cross-sectional study

Published in Neurolmage Clinical 2020; 27, 102347

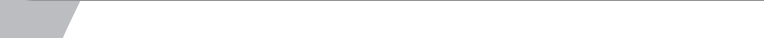

Simone JT van Montfort

Arjen JC Slooter

Ilse MJ Kant

Rutger R van der Leur

Claudia D Spies

Jeroen de Bresser

Theodoor D Witkamp

Jeroen Hendrikse

Edwin van Dellen

On behalf of the BioCog Consortium 


\section{Abstract}

Delirium, the clinical expression of acute encephalopathy, is a common neuropsychiatric syndrome that is related to poor outcomes, such as longterm cognitive impairment. Disturbances of functional brain networks are hypothesized to predispose for delirium. The aim of this study in non-delirious elderly individuals was to investigate whether predisposing risk factors for delirium are associated with $\mathrm{fMRI}$ network characteristics that have been observed during delirium. As predisposing risk factors, we studied age, alcohol misuse, cognitive impairment, depression, functional impairment, history of transient ischemic attack or stroke, and physical status. In this multicenter study, we included 554 subjects and analyzed resting-state fMRI data from 222 elderly subjects (63\% male, age range: 65-85 year) after rigorous motion correction. Functional network characteristics were analyzed and based on the minimum spanning tree (MST). Global functional connectivity strength, network efficiency (MST diameter) and network integration (MST leaf fraction) were analyzed, as these measures were altered during delirium in previous studies. Linear regression analyses were used to investigate the relation between predisposing delirium risk factors and delirium-related $\mathrm{fMRI}$ characteristics, adjusted for confounding and multiple testing. Predisposing risk factors for delirium were not associated with delirium-related $\mathrm{fMRI}$ network characteristics. Older age within our elderly cohort was related to global functional connectivity strength ( $\beta=.182, p<0.05)$, but in the opposite direction than hypothesized. Deliriumrelated functional network impairments can therefore not be considered as the common mechanism for predisposition for delirium.

\section{Introduction}

Delirium is an acute and common neuropsychiatric syndrome, affecting $10-50 \%$ of hospitalized elderly patients ${ }^{1}$. The syndrome is by definition a consequence of one or more medical conditions, and predominantly characterized by a disturbance in attention and awareness with additional cognitive deficits ${ }^{2}$. Delirium is a burden for patients and related to poor outcomes, such as long-term cognitive impairment ${ }^{1}$. From an etiological perspective, risk factors for delirium can be distinguished into predisposing factors (i.e. baseline vulnerability for delirium, for example older age), and precipitating factors (i.e. acute changes that trigger the syndrome, for example an infectious disease) ${ }^{3}$. The development of delirium is usually the result of interaction of several different risk factors ${ }^{1,3,4}$.

While such etiological models aim to understand the underlying biological mechanism of risk for delirium, prediction models aim to predict the occurrence of delirium with a certain accuracy irrespective of mechanistic assumptions of causality. Etiological models on delirium have shown a range of relative risk values of predisposing risk factors for delirium (relative risk scores of dementia: 2.3 - 4.7; cognitive impairment: $1.3-4.2$; history of delirium: 3, functional impairment 2.5 - 4.0; visual impairment: 1.1 - 3.5; hearing impairment: 1.3 ; severity of illness or physical status: $1.1-5.6$; depression: 1.2 - 3.2; history of transient ischemic attack or stroke: 1.6; alcohol misuse: 1.4 - 5.7; older age: $1.1-6.6)^{3}$. Relative risk values or etiological fractions of predisposing risk factors are difficult to quantify as they probably consist of an interaction between predisposition and precipitating events (which may even be non-linear) (van Montfort et al., 2019).

The neural substrate of predisposition for delirium remains poorly understood, and is hypothesized to reflect the cumulative effects of aging and physical, cognitive and psychological frailty. Focusing on the shared biological characteristics of predisposing risk factors allows us to increase our understanding of the risk profile of delirium before acute changes, (such as an infectious disease or trauma), occur. 
t has been hypothesized that delirium is a disconnection syndrome, caused by the breakdown of functional brain networks ${ }^{5-8}$. The functional network may represent the communication between different brain regions ${ }^{9}$. Brain network organization can be characterized based on functional connectivity maps, representing the statistical interdependencies of time-series recorded from different brain areas, for example measured with imaging techniques such as functional magnetic resonance imaging $(\mathrm{fMRI}){ }^{10,11}$. It has been shown that during delirium, the network was less efficient organized and less integrated 6,12,13. Although disturbances between several brain regions have been suggested during delirium, the functional connectivity between two specific regions that could be involved in cognition, attention or consciousness, was found to be altered during delirium in two independent studies, i.e. between the posterior cingulate cortex (PCC) and the dorsolateral prefrontal cortex (DLPFC) ${ }^{14,15}$. In addition, a recent review evaluating network studies on delirium and its risk factors suggested that predisposing delirium risk factors are generally associated with decreased global functional connectivity strength ${ }^{8}$. Functional network impairments may therefore be a common mechanism in the pathophysiology of delirium and a possible biological pathway towards vulnerability for delirium. In this way, vulnerability may correspond to a lower threshold for a transition from a healthy state towards encephalopathy with disturbed brain activity that manifests as delirium ${ }^{16}$

Investigating the integrated effect of delirium risk factors on the functional network may support this hypothesis and may lead to a unified understanding of delirium vulnerability associated with a variety of heterogeneous factors. However, a previous study did not show strong relationships between electroencephalography (EEG) (network) characteristics and predisposing risk factors for delirium ${ }^{17}$. $\mathrm{fMRI}$ has a superior spatial resolution compared to EEG, and could be used to integrate functional brain network analysis with neuroanatomical information, such as functional connectivity between specific regions. Analysis of the association between delirium risk factors and fMRI networks may therefore provide important additional information on altered network organization as a common mechanism to explain vulnerability for delirium. Accordingly, we note that rather than studying delirium itself, we specifically studied risk factors for delirium. The aim of the present study was to evaluate the effect of predisposing delirium risk factors on $\mathrm{fMRI}$ network characteristics in an elderly cohort. It was hypothesized that predisposing risk factors for delirium, separate or combined, are associated with delirium-related fMRI network characteristics, i.e. decreased functional connectivity strength, decreased network efficiency and decreased network integration. As a secondary analysis, we evaluated the effect of predisposing delirium risk factors on the regional connection between the PCC and the DLPFC. Although the etiology of delirium is complex and multifactorial, the exact weight or relative risk of independent risk factors is unknown. The inclusion of risk factors was based on a recent high quality review on delirium ${ }^{3}$.

\section{Methods}

\section{Study design and population}

This study is part of the Biomarker Development for Postoperative Cognitive Impairment in the Elderly (BioCog) project at the University Medical Center (UMC) Utrecht and Charité Hospital at Berlin ${ }^{18}$. In the current cross-sectional sub-study, elderly individuals were included, who were non-hospitalized participants scheduled to undergo elective surgery (i.e. orthopedic-, cardiac-, gastro-intestinal-, maxillofacial- or otorhinolaryngologic surgery), as well as participants that were recruited via a local general practitioner. Inclusion criteria were European ancestry, -age of 65 year or over, and signed informed consent for the study. Participants with one or more of the following characteristics were excluded: a life expectancy shorter than a year; an indication for (early) dementia as indicated with a score of 23 or lower on the Mini Mental State Examination (MMSE) ${ }^{19}$; missing fMRI data. fMRI measurements and clinical assessments were performed on the same day.

\section{Clinical assessment}

Risk factors evaluated in this study were based on a high quality review 3. We were not able to investigate all risk factors described in the review, i.e. participants with dementia, history of delirium and unsolved hearing or visual impairment were unavailable, comorbidity was not measured within this study. 
Age

To determine age, the medical records of the participants were used.

\section{Alcohol misuse}

To define alcohol misuse the self-reported Alcohol Use Disorders Identification Test (Audit) was used. The Audit is a validated questionnaire of 10 items that assesses alcohol consumption, drinking behaviors, and alcohol-related problems ${ }^{20,21}$. A cut-off value of 8 points was used to determine alcohol misuse ${ }^{22}$.

\section{Cognitive impairment}

To define cognitive impairment, the total score on the MMSE was used ${ }^{19}$ and studied as continuous variable.

\section{Depression}

To define depression, the Geriatric Depression Scale (GDS) with 15 items was used ${ }^{23,24}$. A score of 6 was used as cut-off to determine depression.

\section{Functional impairment}

Functional impairment was defined with the validated Barthel Index following the Hamburg classification manual ${ }^{25-27}$. The continuous outcome measure was the total score (0-100), where the maximum score of 100 indicates fully independent functional ability.

\section{History of transient ischemic attack or stroke}

To determine history of transient ischemic attack (TIA) or stroke, the medical records of the participants were used. If this information was not available, participants were asked whether they had experienced a TIA or stroke. If either or both were positive, this risk factor was considered present. In addition, cortical, subcortical and lacunar infarcts, were scored based on the STRIVE criteria ${ }^{28}$ by a neuroradiologists (TW or JB) by use of the T1-weighted, the fluid-attenuated inversion recovery (FLAIR) sequence and the diffusion-weighted image (DWI). The final classification of TIA or stroke was based on all available information.

\section{Physical status}

Physical status was defined using the American Society of Anesthesiologists (ASA) classification. The validated ASA score is widely used for the assessment of preoperative physical status ${ }^{29,30}$, ranging from I. healthy; II. mild systematic disease; III severe systematic disease that is not incapacitating; IV. incapacitating systematic disease that is a constant threat to life; to V. moribund status, not expected to survive for 24 hours without surgery ${ }^{31}$. We studied this measure dichotomized, where an ASA-score of I was classified as healthy and an ASA-score of II or higher as unhealthy.

\section{Estimated intelligence coefficient (IQ)}

The validated reading test for adults 'Nederlandse leestest voor volwassenen' (NLV) for the Dutch subjects or the 'Mehrfachwahl-WortschatzIntelligenztest' (MWT-A) for the German subjects was used to estimate premorbid IQ ${ }^{32,33}$. The raw scores were converted to an estimated IQ score.

\section{Image processing}

MRl scans

Imaging was performed on a 3T Achieva (Philips Medical Systems, Best, the Netherlands) scanner in Utrecht and on a 3T TrioTim (Siemens Healthineers, Erlangen, Germany) scanner in Berlin. For the structural scan, a T1weighted 3D Turbo Field Echo (TFE) image or a T1-weighted Magnetization Prepared Rapid Gradient Echo (MPRAGE) image was made, respectively. The sequence parameters of the T1 TFE were: TR $=7.9 \mathrm{~ms}$, TE $=4.5 \mathrm{~ms}$, flip angle $=8^{\circ}, 192$ sagittal slices, voxel size $1 \times 1 \times 1 \mathrm{~mm}$. The sequence parameters of the T1 MPRAGE were: $T R=2500 \mathrm{~ms}, \mathrm{TE}=4.77 \mathrm{~ms}$, flip angle = 70, 192 sagittal slices, voxel size $1 \times 1 \times 1 \mathrm{~mm}$. For the resting-state blood-oxygen-level dependent (BOLD) fMRI ( $r$-fMRI) scan, a T2*-weighted gradient-echo echoplanar imaging (GE-EPI) image was used with the following sequence parameters: $\mathrm{TR}=2000 \mathrm{~ms}, \mathrm{TE}=30 \mathrm{~ms}$, flip angle $=78^{\circ}, 32$ transversal slices, voxel size

$3 \times 3 \times 3,75 \mathrm{~mm}, 238$ volumes in 7 minutes and 55 seconds. The rs-fMRI was made in a dark room and participants were asked to close their eyes and to stay awake. For visual inspection of brain infarcts a FLAIR (TR $=4800$, $\mathrm{TE}=125$, inversion time $=1650 \mathrm{~ms}$ (Utrecht); TR = 4800, TE = 388, inversion 
time $=1800 \mathrm{~ms}$ (Berlin) and DWI (voxel size $=0.96 \times 1.19 \times 4.00 \mathrm{~mm}^{3}$ $\mathrm{TR}=3294, \mathrm{TE}=68 \mathrm{~ms}$ (Utrecht only)) was used.

\section{Preprocessing}

Image preprocessing was performed using the FMRIB's Software Library (FSL) ${ }^{34-36}$. The brain was automatically extracted from the T1-weighted scan ${ }^{37}$. Time series were motion corrected with MCFLIRT ${ }^{38,39}$. Participants with a mean relative displacement larger than $0.2 \mathrm{~mm}$ were excluded ${ }^{40}$. It has been recognized that motion during the fMRI measurement can induce systematically bias inference, therefore additional motion correction is necessary ${ }^{40-44}$. Volumes that exceeded the threshold of 0.2 $\mathrm{mm}$ framewise displacement ${ }^{45}$ were removed and a regression analysis with 36 motion components was done. Motion components were: three voxel-wise displacement parameters and their white matter, cerebrospinal fluid, global time courses, and the quadrates, temporal derivatives and quadrates of the derivatives of these six parameters ${ }^{43}$. Average time series from the cerebral spinal fluid, the white matter and grey matter intensities were determined after tissue segmentation with the FMRIB's Automated Segmentation Tool (FAST) ${ }^{46}$. A band-pass filter $(0.01-0.08 \mathrm{~Hz})$ was applied 43. The functional scan was registered to the high-resolution anatomical image by using rigid registration. The anatomical scan was subsequently matched with the Montreal Neurological Institute (MNI) 152 T1-weighted $2 \mathrm{~mm}$ image in standard space with affine registration. Functional scans were slice-time corrected and spatial smoothed to reduce noise $(5 \mathrm{~mm}$ full-width-half-maximum). The first 15 volumes were deleted to ensure stabilized magnetization. If the remaining data was less than 240 seconds, the subject was excluded from further analysis ${ }^{47}$

Connectivity and network analysis

We selected 264 regions putative functional areas that cover the cortical and subcortical brain regions ${ }^{48}$. To estimate 264 regional mean time series, voxel time series within each region were averaged. Functional connectivity was subsequently calculated between all time series pairs using Pearson's correlations, resulting in a $264 \times 264$ functional connectivity matrix for every participant. Minimum spanning tree (MST) network backbones were extracted using Kruskal's algorithm (MATLAB, version R2016b) ${ }^{49}$
Only positive correlations were taken into account as a result of the MST analysis, thus avoiding the problematic interpretation of negative BOLD correlations ${ }^{50,51}$. The MST can be considered as the backbone of the original network, connecting all regions without forming loops ${ }^{50,51}$, which allows a relatively unbiased comparison with another network with the same number of regions ${ }^{50-52}$ (Figure 1). Correlation values of the connectivity matrix were ranked and the highest value was included as the first MST connection using Kruskal's algorithm ${ }^{49}$. The second highest value was then added as an MST connection, until all 264 regions were connected. If adding a connection would result in a loop or triangle, this connection was discarded and the next value was evaluated. Note that formally, a maximum spanning tree was constructed; the highest connectivity values were used to construct the MST as these connections were expected to reflect communication with minimal cost. We refer to the minimum spanning tree or MST throughout this manuscript to be consistent with previous literature using this approach. Since it was previously shown that global functional network connectivity, network efficiency, network organization and the regional connectivity between the PCC and the DLPFC were altered in relation to (risk for) delirium, these outcomes were evaluated in our study ${ }^{6,12-15}$.

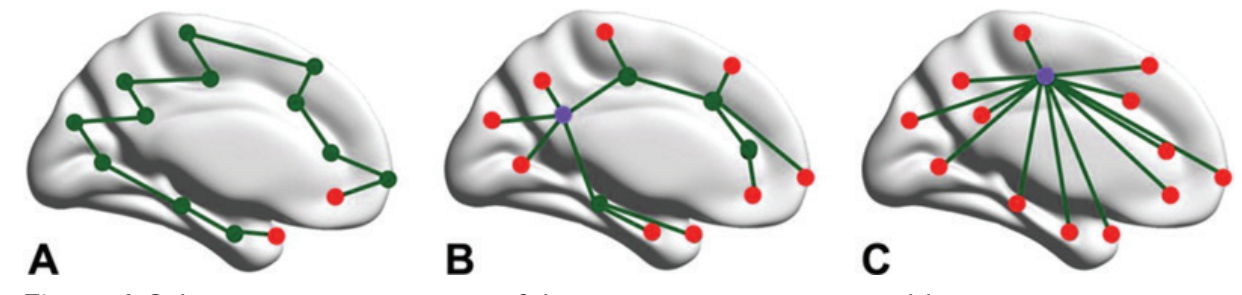

Figure 1: Schematic representation of the minimum spanning tree. Minimum spanning trees can conceptually range between a path-like tree (a less efficient and sparsely integrated network) and a star-like tree (an highly efficient and highly integrated network). Diameter is the length of the path between the two nodes that are furthest apart, and a measure for network efficiency. Leaf fraction is the fraction of leaf nodes (red), i.e. nodes that only have one edge, and therefore a measure of network integration. (A) Line-like network: few leaf nodes and a long diameter, (B) hierarchical tree structure: conceptually optimal topology, (C) star-like network: many leaf nodes + short diameter, central node (purple) will easily be overloaded with information. 


\section{Global functional connectivity strength}

For each participant, global functional connectivity strength was calculated by averaging the connectivity values of the connections in the MST.

Network efficiency (MST diameter)

The MST diameter was used to assess network efficiency. It describes the number of edges connecting the most remote nodes in the MST and gives an indication of the efficiency of global network organization ${ }^{50,51}$. A low diameter describes a network in which information is efficiently processed between remote brain regions $50,51,53$

Network integration (MST leaffraction)

The MST leaf fraction was used to estimate network integration. It describes the proportion of regions with a degree of one, i.e. regions that are connected to only one other region ${ }^{50,51}$. A large leaf fraction describes a network that is highly integrated ${ }^{50,51,53}$.

Regional functional connectivity between the PCC and the DLPFC

The PCC was defined as the region centered at coordinates ( $M N I x / y / z)$ : -11/-56/16 (Power atlas region \#77), -3/-49/13 (Power atlas region \#78) and 11/-54/17 (Power atlas region \#82) ${ }^{14,15,48}$. The DLPFC left was defined as the region centered at coordinates (MNI x/y/z): -42/38/21 (Power atlas region \#167) and -34/55/4 (Power atlas region \#176) ${ }^{14,15,48}$. The DLPFC right was defined as the region centered at coordinates (MNI x/y/z): 38/43/15 (Power atlas region \#168) and 40/18/40 (Power atlas region \#175) 14,15,48. The connection between the PCC and the left or right DLPFC was calculated for each participant using Pearson's correlations between the mean time series of the regions.

\section{Statistical analysis}

Variables that were not normally distributed, were log transformed for all analyses. The association of the individual risk factors on the five outcome measures (i.e. global functional connectivity strength, MST diameter, MST leaf fraction, PCC-DLPFC left and PCC-DLPFC right connectivity strength) were analyzed in separate linear regression models. As age, gender and IQ can be considered as confounders for delirium and network outcomes, we adjusted for center, age (if age was not the determinant), gender and $I Q$ in the analyses ${ }^{1,54-56}$. The associations of all seven risk factors combined on the five outcome measures, adjusted for center, gender and IQ, were studied with three different multivariable linear regression models. To avoid the report of false negative findings, additional, exploratory analyses were performed on the extremes of the distribution of a possible indicator (highest versus lowest quintile).

To control for multiple testing a False Discovery Rate (FDR) correction was applied using the Benjamini and Hochberg method ${ }^{57,58}$. After FDR correction, a corrected $p$-value below 0.05 was considered statistically significant ${ }^{58}$. Statistical analyses were performed in IBM SPSS Statistics version 21.

\section{Results}

Demographics

In this study, 554 participants were eligible (Table 1). From the eligible participants, 17 were excluded due to discontinuation of the fMR measurement, 251 were excluded due to insufficient quality of the fMRI scan because of motion, and 64 were excluded due to missing clinical data. Our total sample therefore consisted of 222 participants with complete data on all clinical variables, of whom 182 were non-hospitalized participants scheduled to undergo elective surgery and 40 were participants recruited via a local general practitioner. Table 2 shows the demographics and risk factors for delirium of the included participants used for analyses. Compared to the total cohort, our study population contained more subjects from the center Utrecht, more males and more subjects that had a history of TIA or stroke, was younger and more healthy (Table 1). No correlation was found between relative motion and global functional connectivity strength, MST diameter or MST leaf fraction (Supplementary Information Figure S1 + Figure S2)

Models of individual risk factors

The results of the models on individual risk factors and the five outcome measures (i.e. global functional connectivity strength, MST diameter, MST 
leaf fraction, functional connectivity strength between PCC and DLPFC left and between PCC and DLPFC right) are shown in Table 3. A significant effect of age on global functional connectivity strength was found ( $F(4$, $216)=5.82, \beta=.178, p=.007, p<0.05$ after FDR correction) (Figure 2), but in the opposite direction than expected. None of the other delirium risk factors were associated with any of the outcome measures. Rerunning our analyses while excluding the participants that were recruited via a local general practitioner revealed the same results.

Models of all risk factors combined

The results of the multivariable models, to test the effect of all seven risk factors on the five outcome measures are also shown in Table 3. Age was positively associated with global functional connectivity strength, independent of other risk factors, center, IQ and gender $(F(10,211)=3.195$, $\beta=.182, p=.008, p<0.05$ after FDR correction). The other multivariable models did not show an association with delirium risk factors. Rerunning our analyses while excluding the participants that were recruited via a local general practitioner revealed the same results.

Post-hoc extreme quintiles comparisons

Comparing the extreme quintiles within the continuous variables (i.e. age, cognitive impairment and functional impairment), showed an association of age on global functional connectivity strength $(t(126)=2.860, p=0.005)$. Other comparisons within the extreme quintiles of the continuous variables did not show statistically significant associations.

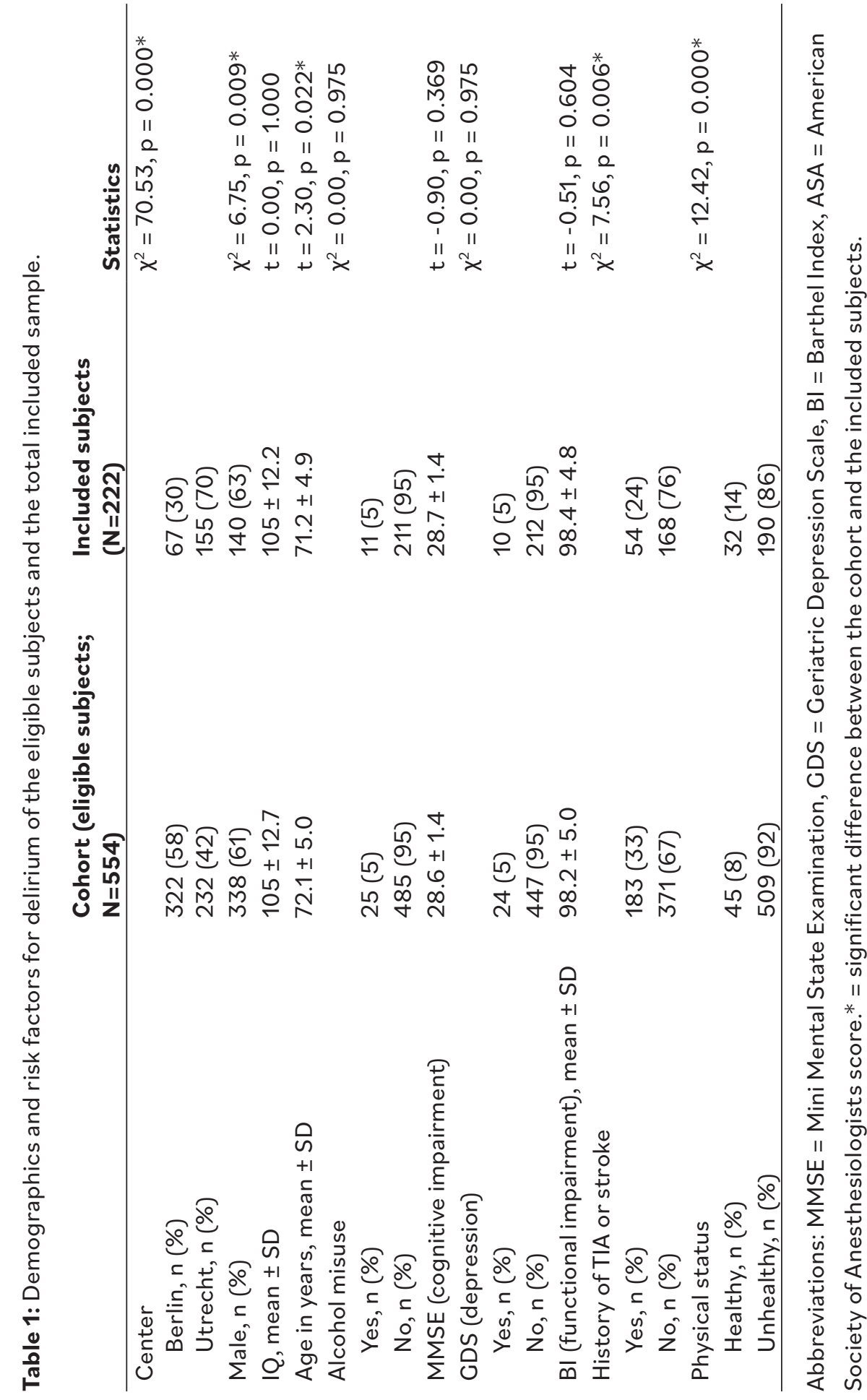



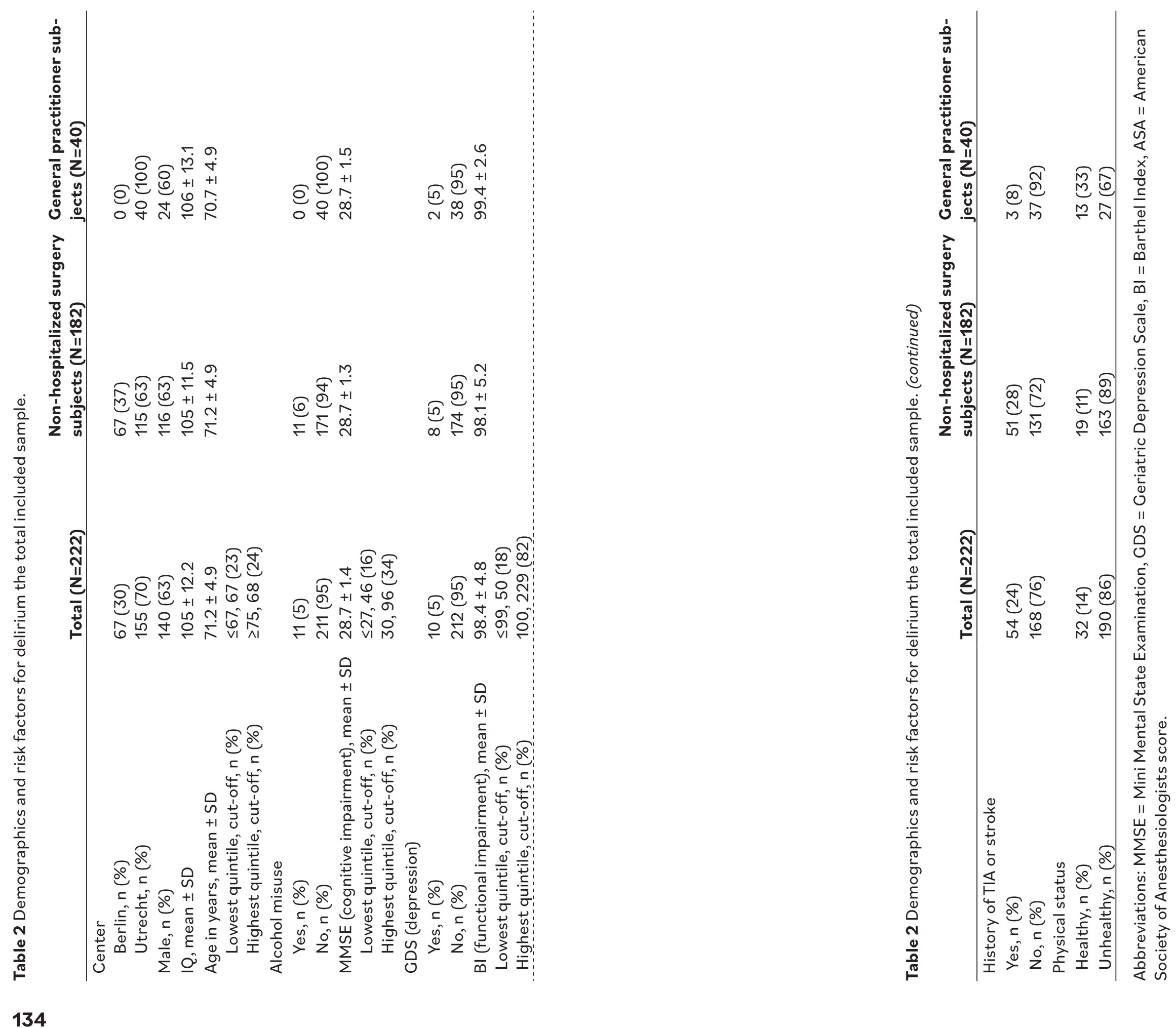

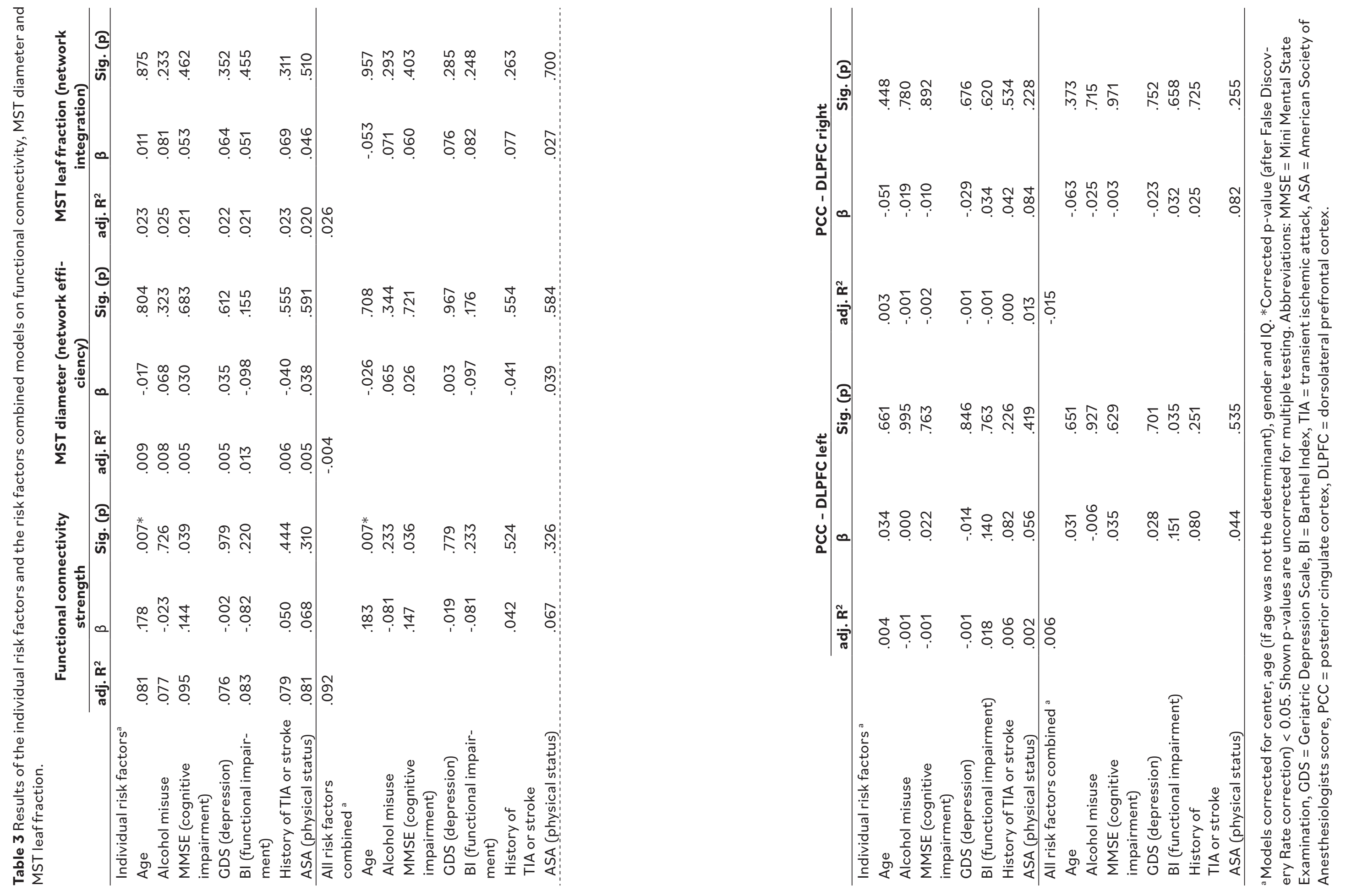


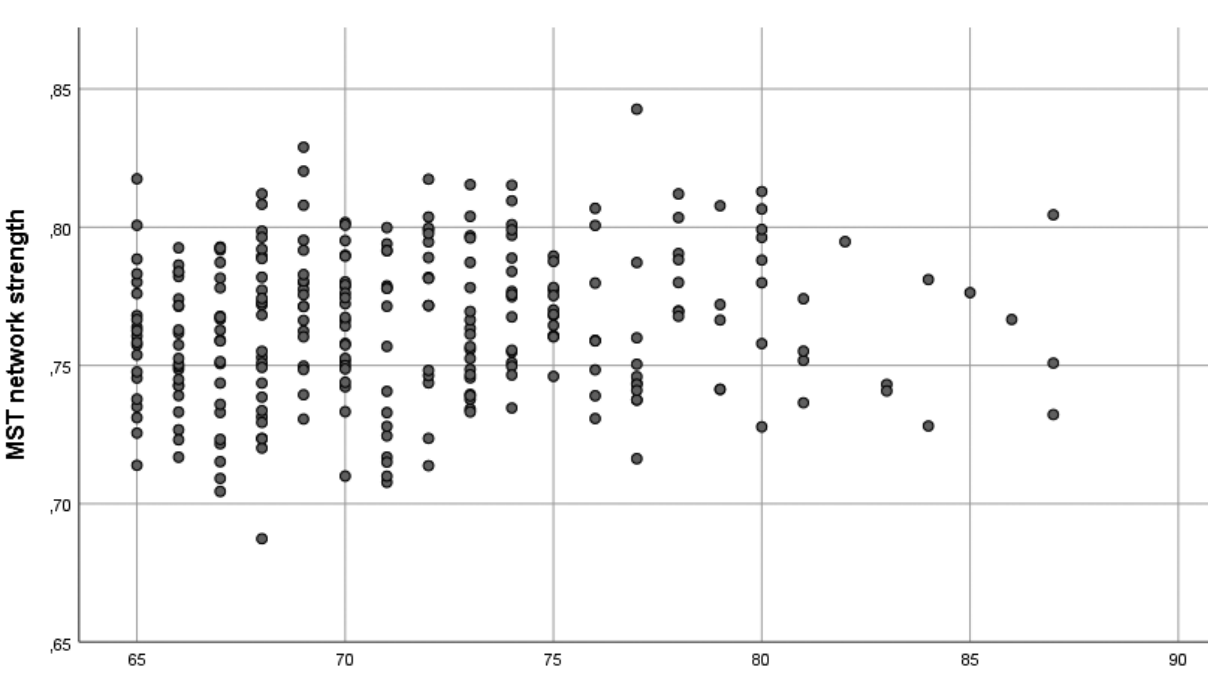

Age

Figure 2 The relationship between age and global functional connectivity strength. A sig nificant association was found between age and global functional connectivity strength. The association was independent of other risk factors for delirium, center, intelligence quotient or gender.

\section{Discussion}

We tested the hypothesis that predisposing risk factors for delirium, separate or combined, are associated with delirium-related fMRI network characteristics, i.e. decreased global functional connectivity strength, decreased network efficiency, decreased network integration, altered regional functional connectivity between the PCC and the DLPFC. None of the studied predisposing risk factors for delirium affected $\mathrm{fMRI}$ network characteristics in the direction of disturbances observed during delirium. In this cohort of elderly subjects, age was related to an increased connectivity strength, which is an association in the opposite direction than hypothesized. No association was found between age and network efficiency or network integration. Therefore, predisposing delirium risk factors seem not to decrease connectivity strength, efficiency or integration of functional brain networks.

The finding of a positive relationship between age and global functional connectivity strength is in contrast with the existing literature, showing decreased global functional connectivity strength when older subjects were compared to younger subjects ${ }^{8,59,60}$. Differences between our results and previous findings may relate to differences in methodology. Previous studies have used other network characteristics and have compared groups of younger subjects to older subjects. We studied age as a continuous variable and included a sample with less contrast, that only contained subjects of 65 years and above, and used MST backbone network characteristics. The MST uses only the backbone network to calculate global functional connectivity strength resulting in connections with a higher signal-tonoise ratio, which may partly explain the differences between our work and previous studies. It should however be noted that the effect size of the association of age with global functional connectivity strength was small, i.e. although the relationship was statistically significant, only a limited proportion of variation was explained by the model. In addition, as patients (especially the oldest part of our sample) should be in a relatively healthy general condition to be scheduled for elective surgery, this finding might be influenced by a cohort effect. Therefore, we cannot draw firm conclusions on the relationship between age and global functional connectivity strength in elderly based on our results.

This study is the first to empirically investigate the association between predisposing risk factors for delirium and delirium-related fMRI network characteristics in the same study population. We used robust methods and included a large number of participants in this multicenter study. However, the selection of the study population may be considered as a limitation of the study. As the selection was performed from a relatively uniform, relatively healthy elderly population, a strong contrast between subjects experiencing risk factors versus subjects not experiencing risk factors was lacking. If the part of the group with delirium risk factors would have been compared to a healthy young group, the results might have differed. Furthermore, the selection of predisposing risk factors was based on an influential review ${ }^{3}$, and not on more recent systematic reviews, as these latter publications included prediction models instead of etiologic models. Prediction models yield predictors that may not necessarily play a role in the pathophysiology of delirium, an example is 'urgent admission'. The interpretation of our study is limited to the included predisposing risk factors. Another limitation is the exclusion of a considerable part of our sample due to strict motion 
correction. In particular, frail elderly may have had problems with laying completely still and may therefore have been excluded from the study. This may have resulted in a selection of a healthier and younger part of the cohort. As motion during $\mathrm{fMRI}$ measurement can induce systematical bias, we were forced to perform this rigorous motion correction ${ }^{41-44}$. Another important limitation of the study is that information on medication use (e.g. psychotropic or antiepileptic drugs) of the subjects during the fMRI measurements was not available. We therefore cannot exclude effects of possible medication on the fMRI measurements. However, the subjects were derived from a relatively healthy non-hospitalized population. Further, we focused on $\mathrm{fMRI}$ network characteristics that are altered during delirium, and did not evaluate the relationship between delirium risk factors and all possible fMRI (network) characteristics or used seed-based analyses to focus on unexplored regional connections that are altered in patients at risk for delirium. It could therefore be that functional brain impairments related to vulnerability to delirium are represented in other $\mathrm{fMRI}$ outcomes. In addition, the structural network was not evaluated in this study. The risk profile for delirium might as well be reflected in structural network abnormalities, as we found in a recent review and meta-analysis ${ }^{8}$.

Our findings are in line with our recent study investigating the association between predisposing risk for delirium and delirium-related neurophysiological alterations using EEG ${ }^{17}$. Taken together, these two studies did not find (strong) evidence for the hypothesis that predisposing risk for delirium is related to the same brain network disturbances as are observed in delirium. Nevertheless, an alternative hypothesis may be that predisposition for delirium is defined by other functional brain (network) characteristics than the profile of delirium itself. In other words, it could be that other parameters reflect a predisposing state than those that are altered during delirium. On the other hand, it could be that predisposing risk for delirium is solely related to structural network abnormalities ${ }^{12,61}$, while precipitating risk factors and the fluctuating nature of delirium itself may be characterized by functional network impairments $6,12,13,62-65$. Predisposing and precipitating risk factors are expected to cause delirium in a complex interaction $3,5,7,66$. The scope of the current work was to test the hypothesis that predisposing risk for delirium is reflected in the functional brain network. Future work should elucidate the predisposing risk of delirium in relation to precipitating events and the occurrence of delirium itself, which are currently subject of study.

\section{Conclusion}

This study was the first to empirically evaluate the hypothesis of functional network impairments as biological pathways underlying vulnerability for delirium using fMRI. None of the predisposing risk factors for delirium was associated with decreased global functional connectivity strength, network efficiency, network integration or the regional functional connectivity between the posterior cingulate and the dorsolateral prefrontal cortex We therefore conclude that predisposition for delirium is not consistently associated to delirium-related functional network alterations, as studied with fMRI.

\section{Acknowledgements}

This work was supported by funding from the European Union Seventh Framework Program under Grant Agreement No. 602461 [FP7/20072013]; and funding from the Dutch Organization for Health Research and Development (ZonMW) Mental Health Program under Grant Agreement No. 60-63600-98-711. 


\section{References}

1. Marcantonio ER. Delirium in Hospitalized Older Adults. Solomon CG, ed. N EnglJ Med. 2017;377(15):1456-1466. doi:10.1056/NEJMcp1605501

2. American Psychiatric Association. Diagnostic and Statistical Manual of Mental Disorders (5th Ed.). Washington, DC; 2013.

3. Inouye SK, Westendorp RG, Saczynski JS. Delirium in elderly people. Lancet 2014;383(9920):911-922. doi:10.1016/S0140-6736(13)60688-1

4. Zaal IJ, Devlin JW, Peelen LM, Slooter AJC. A Systematic Review of Risk Factors for Delirium in the ICU*. Crit Care Med. 2015;43(1):40-47. doi:10.1097/ CCM.0000000000000625

5. Sanders RD. Hypothesis for the pathophysiology of delirium: Role of baseline brain network connectivity and changes in inhibitory tone. Med Hypotheses. 2011;77(1):140143. doi:10.1016/j.mehy.2011.03.048

6. van Dellen E, van der Kooi AW, Numan T, et al. Decreased Functional Connectivity and Disturbed Directionality of Information Flow in the Electroencephalography of Intensive Care Unit Patients with Delirium after Cardiac Surgery. Anesthesiology 2014;121(2):328-335. doi:10.1097/ALN.0000000000000329

7. Young JWS. The network model of delirium. Med Hypotheses. 2017;104:80-85. doi:10.1016/j.mehy.2017.05.027

8. van Montfort SJT, van Dellen E, Stam CJ, et al. Brain network disintegration as a final common pathway for delirium: a systematic review and qualitative meta-analysis. Neurolmage Clin. 2019;23. doi:10.1016/j.nicl.2019.101809

9. Bassett DS, Sporns O. Network neuroscience. Nat Neurosci. 2017;20(3):353-364. doi:10.1038/nn.4502

10. Aertsen AM, Gerstein GL, Habib MK, Palm G. Dynamics of neuronal firing correlation modulation of "effective connectivity." J Neurophysiol. 1989;61(5):900-917. doi:10.1152/ jn.1989.61.5.900

11. van den Heuvel MP, Hulshoff Pol HE. Exploring the brain network: a review on restingstate fMRI functional connectivity. Eur Neuropsychopharmacol. 2010;20(8):519-534. doi:10.1016/j.euroneuro.2010.03.008

12. van Montfort SJT, van Dellen E, van den Bosch AMR, et al. Resting-state fMRI reveals network disintegration during delirium. Neurolmage Clin. 2018;20:35-41. doi:10.1016/J. NICL.2018.06.024

13. Numan T, Slooter AJC, van der Kooi AW, et al. Functional connectivity and network analysis during hypoactive delirium and recovery from anesthesia. Clin Neurophysiol. 2017;128(6):914-924. doi:10.1016/j.clinph.2017.02.022
14. Choi SH, Lee H, Chung TS, et al. Neural network functional connectivity during and after an episode of delirium. Am J Psychiatry. 2012;169(5):498-507. doi:10.1176/appi. ajp.2012.11060976

15. OhJ, Shin JE, Yang KH, et al. Cortical and subcortical changes in resting-state functional connectivity before and during an episode of postoperative delirium. Aust New Zeal Psychiatry. 2019;53(8):794-806. doi:10.1177/0004867419848826

16. Slooter A, Otte W, Devlin J, et al. Updated nomenclature of delirium and acute encephalopathy. Statements endorsed by the American Academy of Neurology (AAN), American Delirium Society (ADS), European Academy of Neurology (EAN), European Delirium Association (EDA), European Geriatric Medici. Intensive Care Med. 2020

17. van Montfort S, van Dellen E, Wattel L, et al. Predisposition for delirium and EEC characteristics. Clin Neurophysiol. 2020

18. Winterer G, Androsova G, Bender O, et al. Personalized risk prediction of postoperative cognitive impairment - rationale for the EU-funded BioCog project. Eur Psychiatry. 2018. doi:10.1016/j.eurpsy.2017.10.004

19. Folstein M, Anthony JC, Parhad I, Duffy B, Gruenberg EM. The meaning of cognitive impairment in the elderly. J Am Geriatr Soc. 1985;33(4):228-235. http://www.ncbi. nlm.nih.gov/pubmed/3989183. Accessed January 28, 2019

20. Bohn MJ, Babor TF, Kranzler HR. The Alcohol Use Disorders Identification Test (AUDIT): validation of a screening instrument for use in medical settings. J Stud Alcohol. 1995;56(4):423-432. doi:10.15288/jsa.1995.56.423

21. Reinert DF, Allen JP. The Alcohol Use Disorders Identification Test: An Update of Research Findings. Alcohol Clin Exp Res. 2007;31(2):185-199. doi:10.1111/j.15300277.2006.00295.x

22. Babor T, Higgins-Biddle J, Saunders J. AUDIT: the alcohol use disorders identification test: guidelines for use in primary health care. 2001. http://apps.who.int/iris/ bitstream/handle/10665/67205/WHO_MSD_?sequence=1. Accessed January 28, 2019.

23. Yesavage JA, Brink TL, Rose TL, et al. Development and validation of a geriatric depression screening scale: A preliminary report.J Psychiatr Res. 1982;17(1):37-49. doi:10.1016/0022-3956(82)90033-4

24. Yesavage JA, Sheikh JI. Geriatric Depression Scale (GDS): Recent evidence and development of a shorter version. Clin Gerontol. 1986;5(1-2):165-173. doi:10.1300/ J018v05n01_09

25. Lübke N, Meinck M, Von Renteln-Kruse W. [The Barthel Index in geriatrics. A context analysis for the Hamburg Classification Manual]. Z Gerontol Geriatr. 2004;37(4):316326. doi:10.1007/s00391-004-0233-2 
26. Collin C, Wade DT, Davies S, Horne V. The Barthel ADL Index: a reliability study. Int Disabil Stud. 1988;10(2):61-63. http://www.ncbi.nlm.nih.gov/pubmed/3403500. Accessed January 28, 2019.

27. Mahoney FI, Barthel DW. FUNCTIONAL EVALUATION: THE BARTHEL INDEX. Md State Med J. 1965;14:61-65. http://www.ncbi.nlm.nih.gov/pubmed/14258950. Accessed January 28, 2019.

28. Wardlaw JM, Smith EE, Biessels GJ, et al. Neuroimaging standards for research into small vessel disease and its contribution to ageing and neurodegeneration. Lancet Neurol. 2013;12(8):822-838. doi:10.1016/S1474-4422(13)70124-8

29. Aronson WL, McAuliffe MS, Miller K. Variability in the American Society of Anesthesiologists Physical Status Classification Scale. AANA J. 2003;71(4):265-274. http://www.ncbi.nlm.nih.gov/pubmed/13677221. Accessed January 28, 2019

30. Sankar A, Johnson SR, Beattie WS, Tait G, Wijeysundera DN. Reliability of the American Society of Anesthesiologists physical status scale in clinical practice. BrJ Anaesth 2014;113(3):424-432. doi:10.1093/bja/aeu100

31. Owens WD, Felts JA, Spitznagel EL. ASA physical status classifications: a study of consistency of ratings. Anesthesiology. 1978;49(4):239-243. http://www.ncbi.nlm. nih.gov/pubmed/697077. Accessed January 28, 2019.

32. Lehrl S, Triebig G, Fischer B. Multiple choice vocabulary test MWT as a valid and short test to estimate premorbid intelligence. Acta Neurol Scand. 1995;91(5):335-345. doi:10.1111/j.1600-0404.1995.tb07018.x

33. Mulder, J., Bouma, J. M. \& Schmand B. Handboek Neuropsychologische Diagnostiek Amsterdam: Pearson Assessment and Information B.V; 2012

34. Jenkinson M, Beckmann CF, Behrens TEJ, Woolrich MW, Smith SM. FSL. Neuroimage. 2012;62(2):782-790. doi:10.1016/j.neuroimage.2011.09.015

35. Smith SM, Jenkinson M, Woolrich MW, et al. Advances in functional and structural MR image analysis and implementation as FSL. In: Neurolmage. Vol 23. ; 2004:S208-S219. doi:10.1016/j.neuroimage.2004.07.051

36. Woolrich MW, Jbabdi S, Patenaude B, et al. Bayesian analysis of neuroimaging data in FSL. Neuroimage. 2009;45(1):S173-S186. doi:10.1016/j.neuroimage.2008.10.055

37. Smith SM. Fast robust automated brain extraction. Hum Brain Mapp. 2002;17(3):143 155. doi:10.1002/hbm.10062

38. Jenkinson M, Bannister P, Brady M, Smith S. Improved Optimization for the Robust and Accurate Linear Registration and Motion Correction of Brain Images. Neuroimage. 2002;17(2):825-841. doi:10.1006/nimg.2002.1132
39. Jenkinson M, Smith S. A global optimisation method for robust affine registration of brain images. Med Image Anal. 2001;5(2):143-156. doi:10.1016/S1361-8415(01)00036

40. Power JD, Barnes KA, Snyder AZ, Schlaggar BL, Petersen SE. Spurious but systematic correlations in functional connectivity MRI networks arise from subject motion. Neuroimage. 2012;59(3):2142-2154. doi:10.1016/j.neuroimage.2011.10.018

41. Power JD, Schlaggar BL, Petersen SE. Recent progress and outstanding issued in motion correction resting state fmri. Neuroimage. 2015;105:536-551. doi:10.1016/j. neuroimage.2014.10.044.Recent

42. Pruim RHR, Mennes M, Buitelaar JK, Beckmann CF. Evaluation of ICA-AROMA and alternative strategies for motion artifact removal in resting state fMRI. Neuroimage. 2015;112:278-287. doi:10.1016/j.neuroimage.2015.02.063

43. Satterthwaite TD, Elliott MA, Gerraty RT, et al. An improved framework for confound regression and filtering for control of motion artifact in the preprocessing of restingstate functional connectivity data. Neuroimage. 2013;64:240-256. doi:10.1016/j neuroimage.2012.08.052

44. Ciric R, Wolf DH, Power JD, et al. Benchmarking of participant-level confound regression strategies for the control of motion artifact in studies of functional connectivity. Neuroimage. 2017;154:174-187. doi:10.1016/j.neuroimage.2017.03.020

45. Power JD, Mitra A, Laumann TO, Snyder AZ, Schlaggar BL, Petersen SE. Methods to detect, characterize, and remove motion artifact in resting state fMRI. Neuroimage. 2014;84:320-341. doi:10.1016/j.neuroimage.2013.08.048

46. Zhang Y, Brady M, Smith S. Segmentation of brain MR images through a hidden Markov random field model and the expectation-maximization algorithm. IEEE Trans Med Imaging. 2001;20(1):45-57. doi:10.1109/42.906424

47. Birn, R.M., Molloy, E.K., Patriat, R., Parker, T., Meier, T.B., Kirk, G.R., Nair, V.A. Meyerand, M.E., Prabhakaran V. The effect of scan length on the reliability of restingstate fMRI connectivity estimates. Neuroimage. 2013;83:550-558. doi:10.1016/J. NEUROIMAGE.2013.05.099

48. Power JD, Cohen AL, Nelson SM, et al. Functional network organization of the human brain. Neuron. 2011;72(4):665-678. doi:10.1016/j.neuron.2011.09.006

49. KruskalJB. On the Shortest Spanning Subtree of a Graph and the Traveling Salesman Problem. Proc Am Math Soc. 1956;7(1):48-50. https://www.jstor.org/stable/ pdf/2033241.pdf. Accessed March 20, 2019 
50. Stam CJ, Tewarie P, Van Dellen E, van Straaten ECW, Hillebrand A, Van Mieghem P. The trees and the forest: Characterization of complex brain networks with minimum spanning trees. Int J Psychophysiol. 2014;92(3):129-138. doi:10.1016/j. ijpsycho.2014.04.001

51. Tewarie P, van Dellen E, Hillebrand A, Stam CJ. The minimum spanning tree: An unbiased method for brain network analysis. Neuroimage. 2015;104:177-188. doi:10.1016/j. neuroimage.2014.10.015

52. van Diessen E, Numan T, van Dellen E, et al. Opportunities and methodological challenges in EEG and MEG resting state functional brain network research. Clin Neurophysiol. 2015;126(8):1468-1481. doi:10.1016/j.clinph.2014.11.018

53. van Dellen E, Bohlken MM, Draaisma L, et al. Structural Brain Network Disturbances in the Psychosis Spectrum. Schizophr Bull. 2016;42(3):782-789. doi:10.1093/schbul/ sbv178

54. Otte WM, van Diessen E, Paul S, et al. Aging alterations in whole-brain networks during adulthood mapped with the minimum spanning tree indices: The interplay of density, connectivity cost and life-time trajectory. Neuroimage. 2015;109:171-189. doi:10.1016/J.NEUROIMAGE.2015.01.011

55. Stumme J, Jockwitz C, Hoffstaedter F, Amunts K, Caspers S. Functional network reorganization in older adults: Graph-theoretical analyses of age, cognition and sex. Neuroimage. 2020:116756. doi:10.1016/j.neuroimage.2020.116756

56. van Dellen E, Sommer IE, Bohlken MM, et al. Minimum spanning tree analysis of the human connectome. Hum Brain Mapp. 2018;39(6):2455-2471. doi:10.1002/hbm.24014

57. Genovese CR, Lazar NA, Nichols T. Thresholding of Statistical Maps in Functiona Neuroimaging Using the False Discovery Rate. Neuroimage. 2002;15(4):870-878. doi:10.1006/NIMG.2001.1037

58. Benjamini Y, Hochberg Y. Controlling the False Discovery Rate: A Practical and Powerful Approach to Multiple Testing. J R Stat Soc Ser B. 1995;57(1):289-300. doi:10.1111/j.2517-6161.1995.tb02031.x

59. Ferreira LK, Regina ACB, Kovacevic N, et al. Aging effects on whole-brain functiona connectivity in adults free of cognitive and psychiatric disorders. Cereb Cortex. 2016;26(9):3851-3865. doi:10.1093/cercor/bhv190

60. Geerligs L, Rubinov M, Cam-Can, Henson RN. State and Trait Components of Functional Connectivity: Individual Differences Vary with Mental State. J Neurosci. 2015;35(41):13949-13961. doi:10.1523/JNEUROSCI.1324-15.2015

61. Kyeong S, Shin JE, Yang KH, Lee WS, Chung T-S, Kim J-J. Neural predisposing factor of postoperative delirium in elderly patients with femoral neck fracture. Sci Rep. 2018;8(1):7602. doi:10.1038/s41598-018-26030-2
62. Blain-Moraes S, Tarnal V, Vanini G, et al. Network Efficiency and Posterior Alpha Patterns Are Markers of Recovery from General Anesthesia: A High-Density Electroencephalography Study in Healthy Volunteers. Front Hum Neurosci. 2017;11:328. doi:10.3389/fnhum.2017.00328

63. Lee H, Mashour GA, Noh G-J, Kim S, Lee U. Reconfiguration of network hub structure after propofol-induced unconsciousness. Anesthesiology. 2013;119(6):1347-1359. doi:10.1097/ALN.0b013e3182a8ec8c

64. Maestú F, Boccaletti S, Castellanos NP, et al. Principles of recovery from traumatic brain injury: Reorganization of functional networks. Neuroimage. 2010;55(3):11891199. doi:10.1016/j.neuroimage.2010.12.046

65. Mashour, GA, Hudetz A. Neural Correlates of Unconsciousness in Large-Scale Brain Networks. Trends Neurosci. 2018;41(3):150-160

66. Maldonado JR. Delirium pathophysiology: An updated hypothesis of the etiology of acute brain failure. Int J Geriatr Psychiatry. 2018;33(11):1428-1457. doi:10.1002/ gps. 4823 


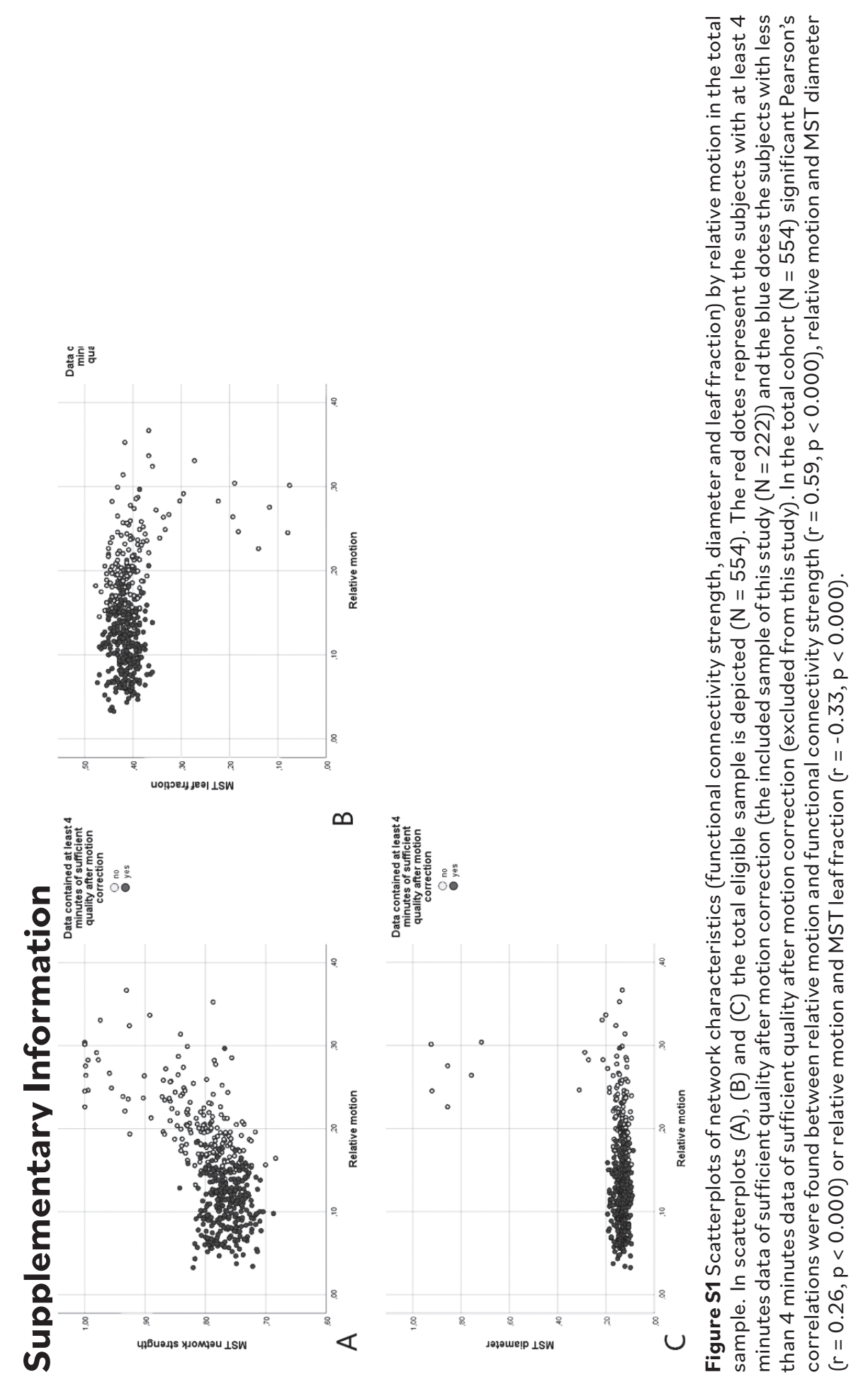

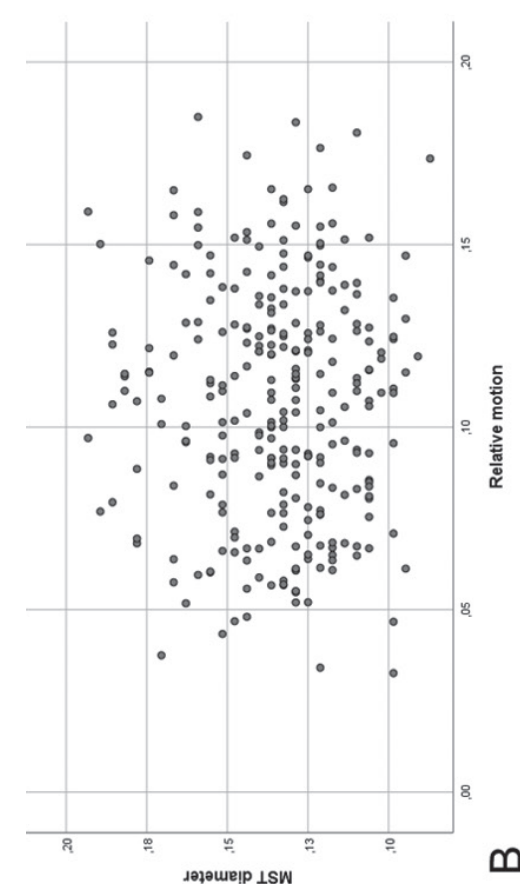
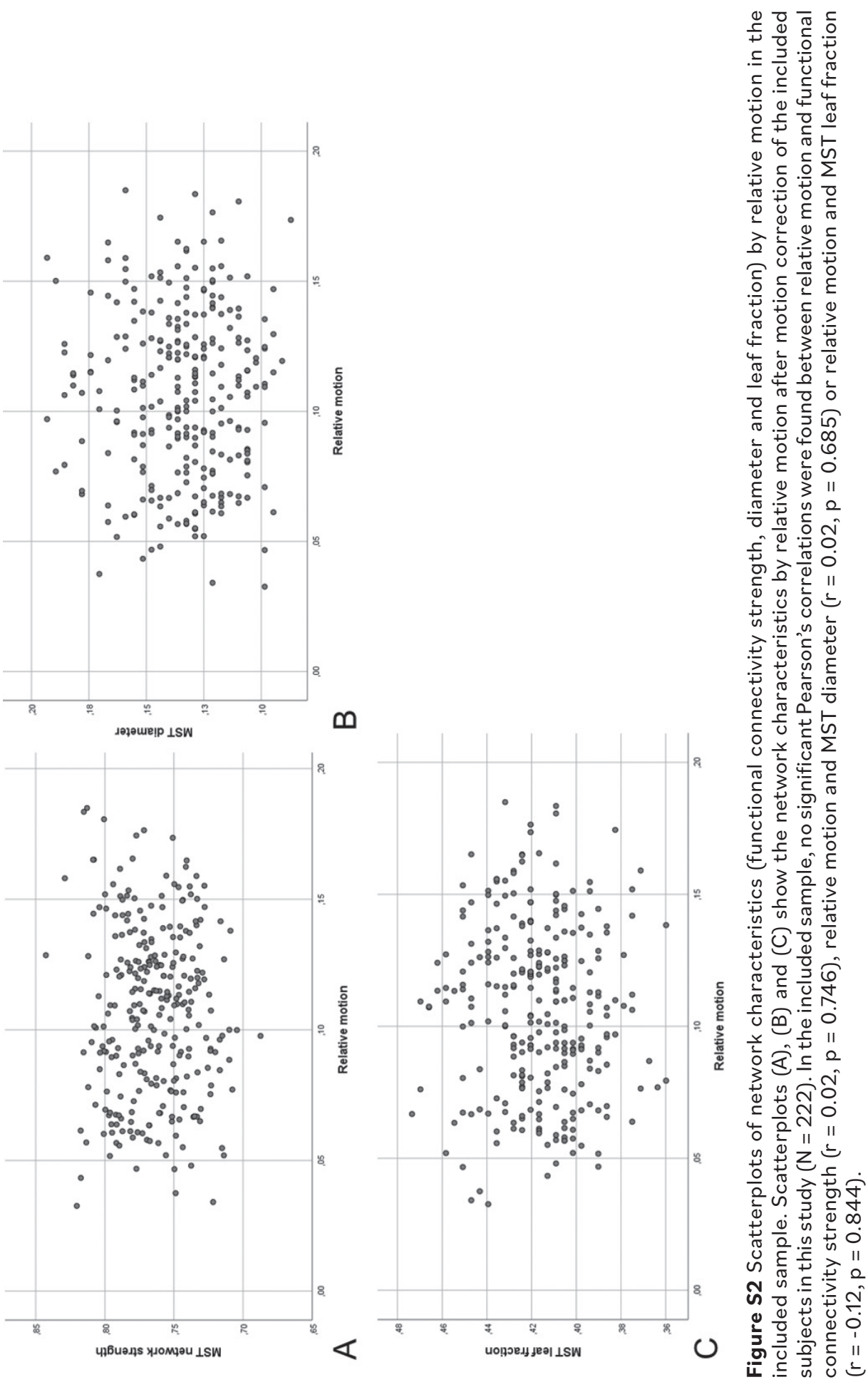


\section{Part 2}

Clinical syndrome of delirium 


\section{Chapter 5}

Resting-state fMRI reveals network disintegration during delirium

Published in Neurolmage Clinical 2018; 20: 35-41

Simone JT van Montfort

Edwin van Dellen

Aletta MR van den Bosch

Willem M Otte

Maya JL Schutte

Soo-Hee Choi

Tae-Sub Chung

Sunghyon Kyeong

Arjen JC Slooter

Jae-Jin Kim 


\section{Abstract}

Delirium is characterized by inattention and other cognitive deficits, symptoms that have been associated with disturbed interactions between remote brain regions. Recent EEG studies confirm that disturbed global network topology may underlie the syndrome, but lack an anatomica basis. The aim of this study was to increase our understanding of the global organization of functional connectivity during delirium and to localize possible alterations. Resting-state fMRI data from 44 subjects were analyzed, and motion-free data were available in nine delirious patients, seven post delirium patients and thirteen non-delirious clinical controls. We focused on the functional network backbones using the minimum spanning tree, which allows unbiased network comparisons. During delirium a longer diameter (mean $(M)=0.30$, standard deviation $(S D)=0.05$, $P=0.024)$ and a lower leaf fraction $(M=0.32, S D=0.03, P=0.027)$ was found compared to the control group $(M=0.28, S D=0.04$ respectively $M=0.35, S D=0.03$ ), suggesting reduced functional network integration and efficiency. Delirium duration was strongly related to loss of network hierarchy ( $r$ o $=-0.92, P=0.001$ ). Connectivity strength was decreased in the post delirium group $(M=0.16, S D=0.01)$ compared to the delirium group $(M=0.17, S D=0.03, P=0.024)$ and the control group $(M=0.19$, $S D=0.02, P=0.001)$. Permutation tests revealed a decreased degree of the right posterior cingulate cortex during delirium and complex regional alterations after delirium. These findings indicate that delirium reflects disintegration of functional interactions between remote brain areas and suggest long-term impact after the syndrome resolves.

\section{Introduction}

Delirium is a common and serious problem affecting more than $15 \%$ of the hospitalized elderly patients ${ }^{1}$. This acute neuropsychiatric syndrome is a direct consequence of another medical condition, and is predominantly characterized by decreased attention and altered awareness with other cognitive disturbances ${ }^{2}$. Delirium is related to poor outcomes such as prolonged length of hospital stay ${ }^{3,4}$ and long-term cognitive impairment

${ }^{5}$. Previous studies suggested neurotransmitter imbalances, an aberrant stress response, and persistent neuroinflammation as potential underlying mechanisms ${ }^{6}$, but the pathogenesis remains poorly understood.

As adequate cognitive functioning requires interaction or functional connectivity between remote brain regions ${ }^{7,8}$, a recent hypothesis stated that delirium is a disconnection syndrome ${ }^{9,10}$, caused by breakdown of functional brain networks ${ }^{11}$. In previous electroencephalography (EEG) studies, we found that delirious patients have a decrease of alpha band (8-13 Hz) global functional connectivity strength, and a more random, less integrated network organization compared to control patients without delirium ${ }^{9,10}$. This was based however on scalp EEG-registrations only, which has low spatial resolution.

Functional magnetic resonance imaging ( $\mathrm{fMRI}$ ) - which maps the entire brain rather than only the surface - could potentially provide important additional information on the anatomical basis of these altered network organization. However, due to the symptomatology of delirium it is very challenging to acquire $\mathrm{fMRI}$ scans from delirious patients. Nonetheless, one fMRI study comparing delirious, post delirious and comparison subjects has been performed, showing abnormal interactions between brain areas involved in attention and awareness in delirious patients ${ }^{12}$. Activity of the dorsolateral prefrontal cortex and the posterior cingulate cortex was positively correlated in delirium patients, whereas a negative correlation was found in comparison participants. Yet this study was focusing on a limited set of a priori defined brain regions. 
Since recent findings suggest that delirium may be an expression of a disrupted global network organization, it would be relevant to extend these finding into a more globally oriented study on network organization. Recent findings strongly suggest that delirium and related cognitive alterations are associated with global network modifications rather than specific regional changes $9,10,13,14$. To understand the nature of delirium it is therefore crucial to also map the global network changes.

Traditional graph analysis has however methodological challenges, including a significant bias in most group comparisons ${ }^{15-17}$. A robust network backbone is captured by the minimum spanning tree (MST). MST analysis has recently been shown to capture clinically relevant network changes in challenging acquisition conditions ${ }^{16,18}$. MSTs connect all nodes with the highest possible weights without forming loops. Therefore, the MST always consists of a fixed number of connections, which avoids the methodological bias of spurious connections or arbitrary thresholding in group comparisons of network topology.

The aim of this study was to increase our understanding of the global organization of functional connectivity after transition to and recovery from delirium, and relate possible alterations to anatomical regions. Secondly, we aimed to explore how network alterations relate to delirium severity measures. We hypothesized that the functional brain network during delirium is less efficient and disintegrated network that partly restores after recovery from delirium.

\section{Methods}

Patient population and clinical assessment

The study protocol was approved by the Institutional Review Board of Yonsei University and informed consent was signed, either given by the participant or a caretaker ${ }^{12}$. Twenty two delirious patients were recruited from the Gangnam Severance Hospital at Yonsei University in Seoul, Korea. Delirium was screened daily and diagnosed according to DSM-IV criteria for delirium by trained psychiatrists using the Memorial Delirium Assessment Scale and the Delirium Rating Scale-Revised-98 (DRS-R-98) ${ }^{19,20}$. Scans obtained from delirium patients included an anatomical T1-weighted scan and a restingstate BOLD fMRI scan. The day after recovery from delirium (i.e. a score of $<10$ on the Memorial Delirium Assessment Scale or a score of $<15$ on the DRS-R-98), a follow-up scan was made of thirteen of these patients using the same scan protocol. The total number of days a patient was diagnosed as delirious was used as a score for delirium duration. The DRS-R-98 score on the day of MRI scanning was used as a measure of severity of delirium. Only low-dose antipsychotics were administered in the delirium and post delirium groups, the dose of antipsychotics was similar in both conditions. The causes of delirium in our sample were multifactorial and mostly included systemic or metabolic disease.

A control group of 22 non-delirious clinical controls, matched on age, sex and extent of leukoaraiosis, was recruited from the Databank for Brain Imaging at Gangnam Severance Hospital. The data bank included functional and structural brain MR images of inpatients or outpatients who had various medical or neurological conditions, who were scanned with the same MR sequences as the patients with delirium and who consented to provide the data.

Exclusion criteria for all groups were a history of cognitive decline such as dementia, a history of seizure or traumatic brain injury, or previously identified focal lesions larger than $3 \mathrm{~cm}$. Since motion artifacts can influence the $\mathrm{FMRI}$ data and cause spurious results ${ }^{21-23}$, a strict motion correction was performed (see additional motion correction paragraph below). After motion correction, our sample consisted of nine delirium patients, of whom seven patients were scanned post delirium and thirteen controls.

Image acquisition

High-resolution anatomical images were obtained using a spoiled gradientecho sequence (matrix $=512 \times 512$, echo time $(T E)=1.7 \mathrm{msec}$, repetition time $(T R)=7.0 \mathrm{msec}$, field of view $=210 \mathrm{~mm}$, slice thickness $=1.2 \mathrm{~mm}$, flip angle $=20^{\circ}$, number of slices $=240$ ) to serve as an anatomical reference. Functional images were obtained over 400 seconds using gradientecho echo-planar imaging sequences in a Signa EXCITE 3-T MR system (GE, Milwaukee; matrix $=64 \times 64$, TE $=17.6 \mathrm{msec}$, $\mathrm{TR}=2500 \mathrm{msec}$, field 
of view $=240 \mathrm{~mm}$, slice thickness $=3 \mathrm{~mm}$, flip angle $=90^{\circ}$, number of slices $=50$ ). All participants were instructed to rest with their eyes closed during each scan.

\section{Preprocessing}

Image preprocessing was performed using the FMRIB's Software Library (FSL) ${ }^{24-26}$. The brain was automatically extracted from the T1-weighted scan ${ }^{27}$. Time series were motion corrected with MCFLIRT ${ }^{28,29}$. This removes subject's head movement and allows calculation of the mean relative displacement. Participants with a mean relative displacement larger than $0.2 \mathrm{~mm}$ were excluded ${ }^{30}$. Six motion components were extracted, i.e. three voxel-wise displacement parameters and the temporal signal intensities obtained from white matter, cerebrospinal fluid, and global time courses ${ }^{23}$. The functional scan was registered to the high-resolution anatomical image by using rigid registration. The anatomical scan was subsequently matched with the Montreal Neurological Institute (MNI) 152 T1-weighted 2 $\mathrm{mm}$ image in standard space with affine registration. Functional scans were slice-time corrected, spatial smoothed to reduce noise $(5 \mathrm{~mm}$ full-widthhalf-maximum). The first 15 volumes were deleted to ensure stabilized magnetization. Average time series from the cerebral spinal fluid, the white matter and grey matter intensities were determined after tissue segmentation Tool (FAST) ${ }^{31}$.

Additional motion correction

It has been recognized that motion can have large impact on the resting state $\mathrm{fMRI}$ signal and can induce systematically bias inference $21,23,30,32,33$.

The standard FSL pipeline is not sufficient to remove motion artifacts from the data, therefore additional motion correction is necessary $21,23,30,32,33$.

Out of two additional motion correction methods, i.e. independent component analysis-based strategy for Automatic Removal of Motion Artifacts (ICA-AROMA) 22,32 and spike regression ${ }^{23,30}$, we selected the most effective one in controlling motion effects in our dataset for final correction, i.e. spike regression (see Supplementary Figure S1). Volumes that exceeded the threshold of $0.2 \mathrm{~mm}$ framewise displacement ${ }^{34}$ were removed and a regression analysis with 24 motion components was done. Motion components were: three voxel-wise displacement parameters and their white matter, cerebrospinal fluid, global time courses, the quadrates, temporal derivatives and quadrates of the derivatives of these six parameters ${ }^{35}$. A band-pass filter $(0.01-0.08 \mathrm{~Hz})$ was applied ${ }^{23}$. If the remaining data was less than 300 seconds, the subject was excluded from further analysis ${ }^{43}$.

\section{Global network analysis}

We selected 90 regions from the AAL brain atlas (V4) that cover the cortical and subcortical regions ${ }^{44}$. To estimate 90 regional mean time series, voxel time series within each AAL region were averaged. A correlation network was constructed for every participant, based on those time series using Pearson's correlation. Only positive correlations were taken into account as a result of the MST analysis, thus avoiding the problematic interpretation of negative BOLD correlations ${ }^{16,18}$. Mean connectivity strength of the network was calculated per subject. MST network backbones were extracted with Kruskal's algorithm ${ }^{45}$ (MATLAB, version R2016b). For each MST the diameter, kappa, tree hierarchy and leaf fraction were calculated (see Table 1 for the definitions and explanation ${ }^{18}$ ). See Figure 1 for an explanation on MST structure.
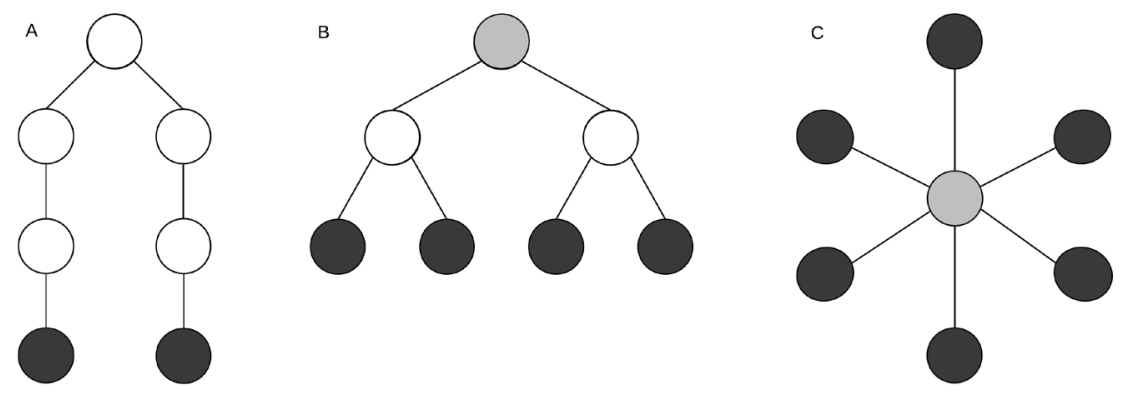

Figure 1 Schematic representation of a line-like, hierarchically tree structure and starlike network. Each network type has 7 nodes and 6 edges. Leaf nodes, the blue nodes in the figure, are nodes that only have one edge and the diameter is the length of the path between the two nodes that are furthest apart. The orange nodes in the figure have a high betweenness centrality. (A) Line-like network: few leaf nodes + long diameter, (B) hierarchical tree structure, (C) star-like network: many leaf nodes + short diameter + a central node with a high betweenness centrality. 
Regional network analysis

To localize possible alterations in network organization to anatomical regions, regional network characteristics were analyzed. Since global network characteristics were analyzed based on the MST, we also focused on MST characteristics for regional analysis, i.e. MST degree and MST betweenness centrality were used ${ }^{18}$ (Table 1 ).

Table 1 Definition and explanation of the MST outcomes

\begin{tabular}{|c|c|c|}
\hline Outcome & Definition & Explanation \\
\hline \multicolumn{3}{|c|}{ Global measures } \\
\hline Diameter & $\begin{array}{l}\text { Number of edges } \\
\text { connecting the most } \\
\text { remote nodes in the } \\
\text { MST }\end{array}$ & $\begin{array}{l}\text { Gives an indication of the efficien- } \\
\text { cy of global network organization. } \\
\text { In a network with a low diameter, } \\
\text { information is efficiently processed } \\
\text { between remote brain regions. }\end{array}$ \\
\hline Kappa & $\begin{array}{l}\text { Measure of the broad- } \\
\text { ness of the degree } \\
\text { distribution }\end{array}$ & $\begin{array}{l}\text { Related to resilience against } \\
\text { attacks, epidemic spreading and } \\
\text { the synchronizability of complex } \\
\text { networks. }\end{array}$ \\
\hline Tree hierarchy & $\begin{array}{l}\text { Quantifies hierarchy as } \\
\text { the trade-off between } \\
\text { large scale integration } \\
\text { in the MST and the } \\
\text { overload of central } \\
\text { nodes }\end{array}$ & $\begin{array}{l}\text { This measure was defined to the } \\
\text { hypothesized optimal topology } \\
\text { of the brain, where information is } \\
\text { transferred between nodes in the } \\
\text { fewest possible steps, while pre- } \\
\text { venting information overload of } \\
\text { central nodes. }\end{array}$ \\
\hline Leaf fraction & $\begin{array}{l}\text { Fraction of leaf nodes } \\
\text { in the MST: a leaf node } \\
\text { is defined as a node } \\
\text { with only one connec- } \\
\text { tion }\end{array}$ & $\begin{array}{l}\text { Describes to what extend the } \\
\text { network has a central, integrated } \\
\text { organization. A high leaf fraction } \\
\text { indicates that the network is largely } \\
\text { dependent on central nodes. }\end{array}$ \\
\hline \multicolumn{3}{|c|}{ Regional measures } \\
\hline Degree & $\begin{array}{l}\text { Number of edges for a } \\
\text { given node }\end{array}$ & $\begin{array}{l}\text { Reflects the importance of a node } \\
\text { in the network. A node with a high } \\
\text { degree is a more important node in } \\
\text { the network. }\end{array}$ \\
\hline $\begin{array}{l}\text { Betweenness } \\
\text { centrality }\end{array}$ & $\begin{array}{l}\text { Fraction of all short- } \\
\text { est paths that pass } \\
\text { through a particular } \\
\text { node }\end{array}$ & $\begin{array}{l}\text { Betweenness centrality ranges } \\
\text { between } 0 \text { (leaf node) and } 1 \text { (central } \\
\text { node in a star-like network). }\end{array}$ \\
\hline
\end{tabular}

Statistical analyses

The mean connectivity strength and the MST outcomes leaf fraction, diameter, kappa and tree hierarchy were compared between i) delirium and controls, ii) post delirium and controls, and iii) delirium and post delirium. For these analyses a bootstrapped linear mixed models with 'group' (i.e. delirium, post delirium, control) as independent variable and 'subject' as random variable were used. This approach was corrected for within-subject correlation allowing calculation of robust $95 \%$ confidence intervals of group differences. Modeling was done within the $\mathrm{R}$ statistical software (version 3) with the Imeresampler package. A False Discovery Rate correction was used to control for multiple comparisons. As exploratory analysis, spearman's correlation analyses were conducted to evaluate the association between the five global MST network outcomes (of the delirium group) and the duration or severity of delirium. These exploratory analyses were not corrected for multiple comparisons.

Regional MST characteristics were tested between groups $(P<.05)$, by using permutation tests in Matlab (Monte Carlo 2-sided test, 1000 permutations), corrected for multiple comparisons ${ }^{46}$. Nodal degree and nodal betweenness centrality of all 90 regions were tested for group differences between i) delirium and controls, ii) post delirium and controls, and iii) delirium and post delirium.

\section{Results}

Demographics

Table 2 shows the demographic and clinical data of the delirium, post delirium and control group. The delirium and control group were comparable on age, sex and extent of leukoaraiosis. The average duration of an episode of delirium was 9.00 days (standard deviation (SD) 2.73). After spike regression, no differences were found in motion, i.e. mean frame-wise displacement, between the three groups $(F=1.35, P=0.60)$. No correlation was found between our network outcome measures and motion, i.e. mean relative displacement (see Supplementary Figure S2). 
Table 2 Demographic and clinical characteristics of delirium patients, the post delirium patients and comparison subjects.

\begin{tabular}{lllll} 
& $\begin{array}{l}\text { Delirium } \\
(\mathbf{N}=9)\end{array}$ & $\begin{array}{l}\text { Post delirium } \\
(\mathbf{N}=7)\end{array}$ & $\begin{array}{l}\text { Control } \\
(\mathbf{N}=13)\end{array}$ & $\mathbf{P}^{1}$ \\
\hline Age (years) & $75.56(6.88)$ & $75.43(8.00)$ & $72.69(6.65)$ & 0.34 \\
Gender (male) & $55.6 \%(\mathrm{~N}=4)$ & $85.7 \%(\mathrm{~N}=6)$ & $46.2 \%(\mathrm{~N}=6)$ & 0.68 \\
PVH (range 0-6) & $2.44(1.33)$ & $2.14(1.22)$ & $1.46(1.39)$ & 0.11 \\
$\begin{array}{l}\text { DWMH (range } \\
\text { 0-24) }\end{array}$ & $7.44(6.32)$ & $4.71(5.90)$ & $7.85(5.47)$ & 0.88 \\
$\begin{array}{l}\text { Focal lesions } \\
\text { (part of group) }\end{array}$ & $55.6 \%(\mathrm{~N}=4)$ & $28.6 \%(\mathrm{~N}=2)$ & $30.8 \%(\mathrm{~N}=4)$ & 0.54 \\
MDAS score & $15.44(3.61)$ & $5.00(2.83)$ & - & - \\
\hline
\end{tabular}

Mean (SD) is shown. ${ }^{1}$ Statistical difference between delirium and control group. Abbreviations: $\mathrm{PVH}=$ periventricular hyperintensities, $\mathrm{DWMH}=$ deep white matter hyperintensities, MDAS = Memorial Delirium Assessment Scale.

\section{Global network organization}

Figure 2 shows boxplots of the overall connectivity strength and MST outcomes diameter, kappa, leaf and tree hierarchy for the delirium, post delirium and control group. Mean network outcomes are displayed in Supplementary Table S1. Connectivity strength was significantly decreased in the post delirium group (M: 0.16, SD: 0.01) compared to the control group (M: 0.19, SD: 0.02) with a difference of -0.04 (95\% Cl -0.05- -0.02 corrected $\mathrm{P}=0.001)$ and compared to the delirium group (M: 0.17, SD: $0.03)$ with a difference of $-0.02(95 \% \mathrm{Cl}-0.02-0.00$, corrected $\mathrm{P}=0.027)$ Diameter was significantly increased during delirium (M: 0.30, SD: 0.05) compared to the control group (M: 0.28, SD: 0.04) with a difference of 0.04 (95\% Cl $-0.01-0.08$, corrected $\mathrm{P}=0.024)$. Leaf fraction was significantly decreased during delirium (M: 0.32, SD: 0.03) compared to the control group (M: 0.35, SD: 0.03), with a difference of - $0.02(95 \% \mathrm{Cl}-0.04-0.02$ corrected $\mathrm{P}=0.027$ ).

In order to increase the comparability with previous studies, we additionally calculated classical graph measures, i.e. clustering coefficient, density and path length from the weighted connectivity matrix (for definitions see Supplementary Table S2). This resulted in similar results as our MST findings, however these analyses additionally showed decreased clustering, decreased density and increased path length in the post delirium group (Supplementary Figure S3).

We found significant negative correlations between delirium duration and leaf fraction (rho $=-0.73, \mathrm{P}=0.039$ ), and between delirium duration and tree hierarchy (rho $=-0.92, \mathrm{P}=0.001$ ) (Figure 2). No significant correlations were found between delirium severity and the global MST network measures.

Regional network organization

We performed permutation analysis to test for possible alterations of the nodal characteristics degree and betweenness centrality. We found that the degree of the right posterior cingulate cortex was lower in the delirium group compared to the control group (corrected $P=0.039$ ) (Figure 3 ).

Several differences in regional betweenness centrality were found between groups (Supplementary Figure S4). Betweenness centrality of the right inferior temporal gyrus was lower in the delirium group compared to the control group (corrected $\mathrm{P}=0.004$ ). Betweenness centrality of the left anterior cingulum and the right pallidum were lower in the post delirium group compared to the control group (both corrected $P=0.016$ ) Betweenness centrality of the orbital part of the right middle frontal gyrus, the right medial orbitofrontal cortex and the left anterior cingulate were lower in the delirium group compared to the post delirium group (corrected $P=0.030$, corrected $P=0.016$, corrected $P=0.031$, resp.) 


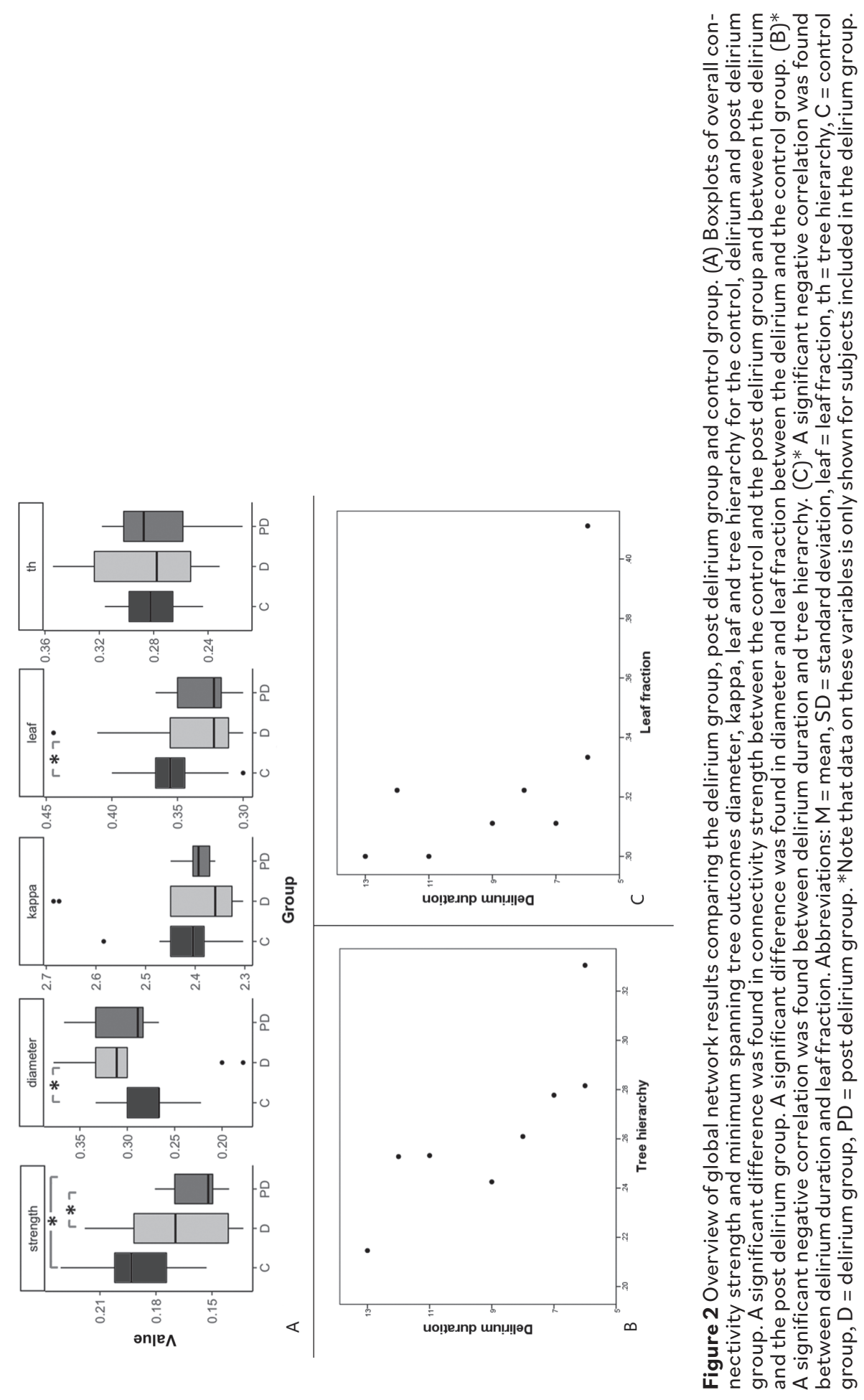

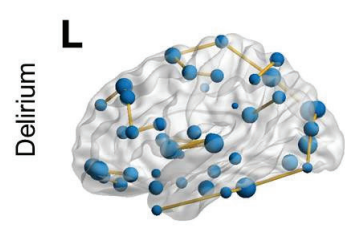
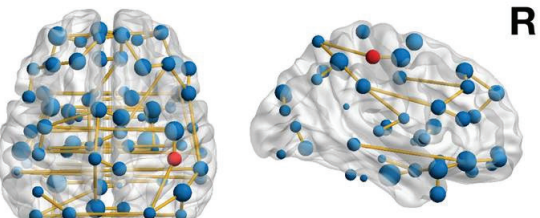

$\mathbf{R}$
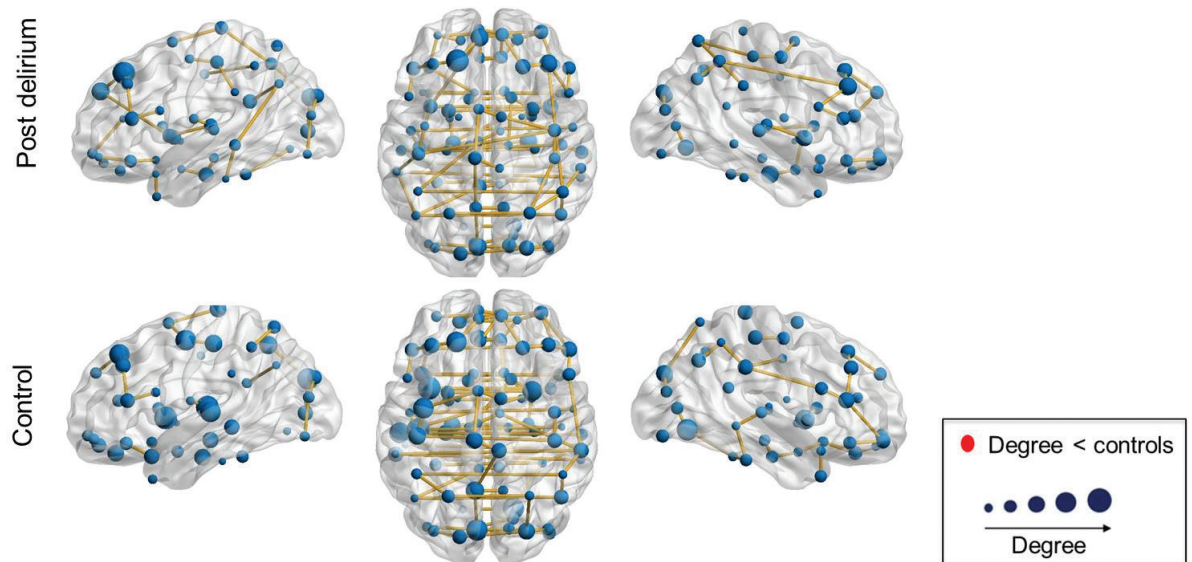

Figure 3 Visualization of the mean degree in the minimum spanning tree network of the delirium, post delirium and the control group. The anatomical labeling atlas with 90 regions was used. The size of the nodes corresponds to the degree. Red nodes mark regions with a significant group difference in degree. The degree of the right posterior cingulate was significantly lower in the delirium group compared to the control group.

\section{Discussion}

This study shows a less efficient, disintegrated network during delirium, which was in accordance with our hypothesis. More profound network disintegration was strongly associated with longer duration of delirium, suggesting that this mechanism is pivotal in the pathophysiology of the syndrome. Connectivity strength was declined after recovery of delirium. In addition, several differences between regional network characteristics were found after delirium was resolved, including a lower degree of the right posterior cingulate cortex and a lower centrality of the right inferior temporal gyrus during delirium and complex regional alterations after delirium. Taken together, these findings suggest long-term impact of delirium on functional connectivity and brain network organization after remission of the syndrome. 
Our findings of decreased network integration during delirium are in line with previous EEG graph theoretical studies ${ }^{9,10}$. The loss of network integration and efficiency may reflect the underlying neural mechanism for the cognitive dysfunction during the syndrome. Interestingly, the transition to a more path-like network during delirium is similar to findings in MST studies on dementia ${ }^{14,47}$. Previous EEG studies additionally suggested a change in connectivity strength during delirium ${ }^{9,10}$. In the present study we found a similar trend, but this did not reach statistical significance. This may be explained by our small sample size. Another possibility may be that $\mathrm{fMRI}$ is less sensitive than EEG in picking up functional connectivity strength alterations during delirium. Nevertheless, we did find a difference in connectivity strength between the post delirium group and the contro group, and between the post delirium and the delirium group. Delirium is associated with long-term cognitive decline ${ }^{5,48,49}$, while decreased functional connectivity was related to cognitive impairment in previous work 14,47 . We speculate that an irreversible decrease of connectivity strength due to delirium may be an underlying pathophysiological mechanism of this association between delirium and cognitive decline.

Although classical graph analyses showed persisting organizational changes after delirium was resolved, MST network analyses did not. This difference might be due to the strong dependency of classical graph measures on connectivity strength, which makes the interpretation of the classical graph measures difficult ${ }^{17}$. As simulation studies suggest that the MST overcomes this bias ${ }^{18}$, we will focus on the MST findings in the evaluation of network alteration after delirium. Interestingly, we found a large variation in post delirium patients in MST outcomes that were affected during delirium. This may be due to the smaller size of the group, but could also indicate heterogeneity. Considering that delirium is associated with long-term cognitive impairment ${ }^{5,48,49}$, it could be that the sub-group of delirious patients who will develop this long-term problems shows remaining alterations in global brain organization, while the patients without lasting problems will recover from global brain organization disturbances. In line with this hypothesis, we observed that network integration was negatively associated with delirium duration. The post delirium patients also showed regional network alterations compared to the control group, which may reflect (more localized) long-term network impairments.

This fMRI study showed a lower nodal MST degree, i.e. number of connections, in the right posterior cingulate cortex during delirium. The posterior cingulate cortex is an important structure in the default mode network and previous studies have shown impaired activity in this region in patient groups with disorders of cognition, attention or consciousness ${ }^{50}$. A previous $\mathrm{fMRI}$ study on network alteration during delirium in a priori chosen brain regions, showed an abnormal interaction between the posterior cingulate cortex and the dorsolateral prefrontal cortex, i.e. regions involved in attention and cognition, which resolved after delirium. The lower degree in the right posterior cingulate cortex might reflect loss of hub function of this region, related to a development of long-term cognitive impairment.

We observed that betweenness centrality of post delirium patients was lower in a part of the default mode network, i.e. the middle prefrontal cortex, and in regions involved in emotional and cognitive processes, i.e. the orbitofrontal cortex, anterior cingulate and globus pallidus. These regions are not known in the literature as hub regions ${ }^{51}$. We would therefore not expect a high betweenness centrality, which makes it difficult to interpret what the importance of a lower betweenness centrality in the post delirium group is. The complex regional alterations might reflect a process of neural plasticity in order to recover from delirium, or might be an indication of lasting impairment related to the vulnerability for negative outcomes after delirium.

Strengths of our study were the strict methodology, using groups matched on age, sex and the extent of leukoaraiosis, strict motion correction and bias limiting MST network comparisons. Furthermore, we had access to a unique dataset of $\mathrm{fMRI}$ scans during delirium, which is very challenging to perform.

This study also has several limitations. It is difficult to instruct delirious, sometimes restless, patients, to undergo resting-state fMRI measurements. Therefore, the reliability of the 'resting-state' may not be assured in this study. Due to the limited scanning time and the need for strict motion 
correction for global brain network analysis, a considerable part of our study population had to be excluded. It can be hypothesized that the most restless patients that needed to be excluded were the patients with most severe delirium. This would have resulted in an underestimation of the true effect. Samples and preprocessing steps may differ from the previous study on this dataset ${ }^{12}$, due to recent improvements in processing of resting state $\mathrm{fMRI}$ data for connectivity analysis. Another limitation might be that antipsychotic medication could have influenced our results ${ }^{52}$. It should however be noted that the use of antipsychotics was equally distributed between the delirium and post delirium scans and that only low-dose antipsychotics were administered in both scan conditions. Future research should focus on global and regional network studies during delirium, with larger sample sizes and follow-up measurements to evaluate the long-term cognitive effects of network changes due to delirium.

\section{Conclusion}

This fMRI study revealed a disintegrated, less efficient resting-state network during delirium, which correlates with the duration of the disorder, and loss of hub function of the right posterior cingulate cortex. Connectivity strength was declined after delirium was resolved. Additional complex regional alterations were shown after delirium, indicating a process of persistent vulnerability or ongoing recovery. These findings provide further evidence that delirium reflects a disintegration of functional interactions between remote brain areas and indicate long-term impact after remission of the syndrome.

\section{Acknowledgements}

This work was supported by a grant from the Korea Health Technology R\&D Project through the Korea Health Industry Development Institute (KHIDI), funded by the Ministry of Health \& Welfare, Republic of Korea (grant number: HI16C0132)

\section{References}

1. Marcantonio ER. Delirium in Hospitalized Older Adults. Solomon CG, ed. N EnglJ Med. 2017;377(15):1456-1466. doi:10.1056/NEJMcp1605501.

2. American Psychiatric Association. Diagnostic and Statistical Manual of Mental Disorders. American Psychiatric Association; 2013. doi:10.1176/appi.books.9780890425596.

3. Brooks PB. Postoperative Delirium in Elderly Patients. AJN, AmJ Nurs. 2012;112(9):38 49. doi:10.1097/01.NAJ.0000418922.53224.36.

4. Pompei P, Foreman M, Rudberg MA, Inouye SK, Braund V, Cassel CK. Delirium in Hospitalized Older Persons: Outcomes and Predictors. J Am Geriatr Soc 1994;42(8):809-815. doi:10.1111/j.1532-5415.1994.tb06551.x.

5. Pandharipande PP, Girard TD, Jackson JC, et al. Long-term cognitive impairment after critical illness. N EnglJ Med. 2013;369(14):1306-1316. doi:10.1056/NEJMoa1301372.

6. Zaal IJ, Devlin JW, Peelen LM, Slooter AJC. A Systematic Review of Risk Factors for Delirium in the ICU*. Crit Care Med. 2015;43(1):40-47. doi:10.1097/ CCM.0000000000000625

7. Bullmore E, Sporns O. Complex brain networks: graph theoretical analysis of structural and functional systems. Nat Rev Neurosci Nat Rev Neurosci. 2009;10(3):186-198. doi:10.1038/nrn2575

8. Stam CJ, van Straaten ECW. The organization of physiological brain networks. Clin Neurophysiol. 2012;123(6):1067-1087. doi:10.1016/j.clinph.2012.01.011.

9. van Dellen E, van der Kooi AW, Numan T, et al. Decreased Functional Connectivity and Disturbed Directionality of Information Flow in the Electroencephalography of Intensive Care Unit Patients with Delirium after Cardiac Surgery. Anesthesiology. 2014;121(2):328-335. doi:10.1097/ALN.0000000000000329.

10. Numan T, Slooter AJC, van der Kooi AW, et al. Functional connectivity and network analysis during hypoactive delirium and recovery from anesthesia. Clin Neurophysiol. 2017;128(6):914-924. doi:10.1016/j.clinph.2017.02.022.

11. Sanders RD. Hypothesis for the pathophysiology of delirium: Role of baseline brain network connectivity and changes in inhibitory tone. Med Hypotheses. 2011;77(1):140143. doi:10.1016/j.mehy.2011.03.048.

12. Choi SH, Lee H, Chung TS, et al. Neural network functional connectivity during and after an episode of delirium. Am J Psychiatry. 2012;169(5):498-507. doi:10.1176/appi. ajp.2012.11060976.

13. Stam CJ. Modern network science of neurological disorders. Nat Rev Neurosci. 2014;15(10):683-695. doi:10.1038/nrn3801. 
14. van Dellen E, de Waal H, van der Flier WM, et al. Loss of EEG Network Efficiency Is Related to Cognitive Impairment in Dementia With Lewy Bodies. Mov Disord. 2015;30(13):1785-1793. doi:10.1002/mds.26309.

15. Fornito A, Zalesky A, Breakspear M. Graph analysis of the human connectome Promise, progress, and pitfalls. Neuroimage. 2013;80:426-444. doi:10.1016/j. neuroimage.2013.04.087.

16. Stam CJ, Tewarie P, Van Dellen E, van Straaten ECW, Hillebrand A, Van Mieghem P. The trees and the forest: Characterization of complex brain networks with minimum spanning trees. Int J Psychophysiol. 2014;92(3):129-138. doi:10.1016/j ijpsycho.2014.04.001.

17. van Wijk BCM, Stam CJ, Daffertshofer A, Stam C, Apkarian A. Comparing Brain Networks of Different Size and Connectivity Density Using Graph Theory. Sporns O, ed. PLoS One. 2010;5(10):e13701. doi:10.1371/journal.pone.0013701.

18. Tewarie P, van Dellen E, Hillebrand A, Stam CJ. The minimum spanning tree: An unbiased method for brain network analysis. Neuroimage. 2015;104:177-188. doi:10.1016/j. neuroimage.2014.10.015.

19. Breitbart W, Rosenfeld B, Roth A, Smith MJ, Cohen K, Passik S. The memorial delirium assessment scale.J Pain Symptom Manage. 1997;13(3):128-137. doi:10.1016/S08853924(96)00316-8.

20. Trzepacz PT, Mittal D, Torres R, Kanary K, Norton J, Jimerson N. Validation of the Delirium Rating Scale-Revised-98. J Neuropsychiatry Clin Neurosci. 2001;13(2):229 242. doi:10.1176/jnp.13.2.229.

21. Power JD, Schlaggar BL, Petersen SE. Recent progress and outstanding issued in motion correction resting state fmri. Neuroimage. 2015;105:536-551. doi:10.1016/j. neuroimage.2014.10.044

22. Pruim RHR, Mennes M, van Rooij D, Llera A, Buitelaar JK, Beckmann CF. ICA-AROMA: A robust ICA-based strategy for removing motion artifacts from $\mathrm{fMRI}$ data. Neuroimage. 2015;112:267-277. doi:10.1016/j.neuroimage.2015.02.064

23. Satterthwaite TD, Elliott MA, Gerraty RT, et al. An improved framework for confound regression and filtering for control of motion artifact in the preprocessing of restingstate functional connectivity data. Neuroimage. 2013;64:240-256. doi:10.1016/j. neuroimage.2012.08.052

24. Jenkinson M, Beckmann CF, Behrens TEJ, Woolrich MW, Smith SM. FSL. Neuroimage. 2012;62(2):782-790. doi:10.1016/j.neuroimage.2011.09.015.

25. Smith $\mathrm{SM}$, Jenkinson M, Woolrich MW, et al. Advances in functional and structura MR image analysis and implementation as FSL. Neurolmage. 2004;23:S208-S219. doi:10.1016/j.neuroimage.2004.07.051
26. Woolrich MW, Jbabdi S, Patenaude B, et al. Bayesian analysis of neuroimaging data in FSL. Neuroimage. 2009;45(1):S173-S186. doi:10.1016/j.neuroimage.2008.10.055

27. Smith SM. Fast robust automated brain extraction. Hum Brain Mapp. 2002;17(3):143 155. doi:10.1002/hbm.10062

28. Jenkinson M, Bannister P, Brady M, Smith S. Improved Optimization for the Robust and Accurate Linear Registration and Motion Correction of Brain Images. Neuroimage. 2002;17(2):825-841. doi:10.1006/nimg.2002.1132.

29. Jenkinson M, Smith S. A global optimisation method for robust affine registration of brain images. Med Image Anal. 2001;5(2):143-156. doi:10.1016/S1361-8415(01)000366.

30. Power JD, Barnes KA, Snyder AZ, Schlaggar BL, Petersen SE. Spurious but systematic correlations in functional connectivity MRI networks arise from subject motion. Neuroimage. 2012;59(3):2142-2154. doi:10.1016/j.neuroimage.2011.10.018.

31. Zhang Y, Brady M, Smith S. Segmentation of brain MR images through a hidden Markov random field model and the expectation-maximization algorithm. IEEE Trans Med Imaging. 2001;20(1):45-57. doi:10.1109/42.906424

32. Pruim RHR, Mennes M, Buitelaar JK, Beckmann CF. Evaluation of ICA-AROMA and alternative strategies for motion artifact removal in resting state fMRI. Neuroimage. 2015;112:278-287. doi:10.1016/j.neuroimage.2015.02.063

33. Ciric $R$, Wolf $D H$, Power JD, et al. Benchmarking of participant-level confound regression strategies for the control of motion artifact in studies of functional connectivity. Neuroimage. 2017;154:174-187. doi:10.1016/j.neuroimage.2017.03.020.

34. Power JD, Mitra A, Laumann TO, Snyder AZ, Schlaggar BL, Petersen SE. Methods to detect, characterize, and remove motion artifact in resting state fMRI. Neuroimage. 2014;84:320-341. doi:10.1016/j.neuroimage.2013.08.048

35. Yan C-C, Cheung B, Kelly C, et al. A comprehensive assessment of regional variation in the impact of head micromovements on functional connectomics. Neuroimage. 2013;76:183-201. doi:10.1016/j.neuroimage.2013.03.004.

36. Niazy RK, Xie J, Miller K, Beckmann CF, Smith SM. Spectral characteristics of resting state networks. Prog Brain Res. 2011;193:259-276. doi:10.1016/B978-0-444-538390.00017-X.

37. Dijk KRA Van, Hedden T, Venkataraman A, Evans KC, Lazar SW, Buckner RL. Intrinsic Functional Connectivity As a Tool For Human Connectomics: Theory, Properties, and Optimization. J Neurophysiol. 2010;103:297-321. doi:10.1152/jn.00783.2009.

38. Feinberg DA, Moeller S, Smith SM, et al. Multiplexed Echo Planar Imaging for SubSecond Whole Brain FMRI and Fast Diffusion Imaging. PLoS One. 2010;5(12):e15710. doi:10.1371/journal.pone.0015710. 
39. Smith SM, Beckmann CF, Andersson J, et al. Resting-state fMRI in the Human Connectome Project. Neuroimage. 2013;80:144-168. doi:10.1016/j neuroimage.2013.05.039

40. Della-Maggiore, V., Chau, W., Peres-Neto, P.R., Mclntosh AR. An Empirical Comparison of SPM Preprocessing Parameters to the Analysis of fMRI Data. Neuroimage. 2002;17(1):19-28. doi:10.1006/NIMG.2002.1113.

41. Davey, C.E., Grayden, D.B., Egan, G.F., Johnston LA. Filtering induces correlation in fMRI resting state data. Neuroimage. 2013;64:728-740. doi:10.1016/j. neuroimage.2012.08.022

42. Christova P, Lewis SM, Jerde TA, Lynch JK, Georgopoulos AP. True association between resting fMRI time series based on innovations.J Neural Eng. 2011;8(4):46025. doi:10.1088/1741-2560/8/4/046025

43. Birn, R.M., Molloy, E.K., Patriat, R., Parker, T., Meier, T.B., Kirk, G.R., Nair, V.A., Meyerand, M.E., Prabhakaran V. The effect of scan length on the reliability of resting state fMRI connectivity estimates. Neuroimage. 2013;83:550-558. doi:10.1016/j. neuroimage.2013.05.099

44. Tzourio-Mazoyer N, Landeau B, Papathanassiou D, et al. Automated Anatomical Labeling of Activations in SPM Using a Macroscopic Anatomical Parcellation of the MNI MRI Single-Subject Brain. Neuroimage. 2002;15(1):273-289. doi:10.1006/ nimg.2001.0978.

45. Kruskal JB,Jr. On the Shortest Spanning Subtree of a Graph and the Traveling Salesman Problem. Proc Am Math Soc. 1956;7(1):48. doi:10.2307/2033241.

46. Groppe DM, Urbach TP, Kutas M. Mass univariate analysis of event-related brain potentials/fields I: a critical tutorial review. Psychophysiology. 2011;48(12):1711-1725 doi:10.1111/j.1469-8986.2011.01273.x.

47. Yu M, Gouw AA, Hillebrand A, et al. Different functional connectivity and network topology in behavioral variant of frontotemporal dementia and Alzheimer's disease: an EEG study. Neurobiol Aging. 2016;42:150-162. doi:10.1016/j. neurobiolaging.2016.03.018.

48. Inouye SK, Marcantonio ER, Kosar CM, et al. The short-term and long-term relationship between delirium and cognitive trajectory in older surgical patients. Alzheimer's Dement. 2016;12(7):766-775. doi:10.1016/j.jalz.2016.03.005.

49. Wolters AE, Peelen LM, Veldhuijzen DS, et al. Long-Term Self-Reported Cognitive Problems After Delirium in the Intensive Care Unit and the Effect of Systemic Inflammation. J Am Geriatr Soc. 2017;65(4):786-791. doi:10.1111/jgs.14660.

50. Leech R, Sharp DJ. The role of the posterior cingulate cortex in cognition and disease. Brain. 2014;137(1):12-32. doi:10.1093/brain/awt162.
51. Heuvel M van den, Sporns O. Network hubs in the human brain. Trends Cogn Sci. 2013;17(12):683-696. doi: 10.1016/j.tics.2013.09.012.

52. H. Roder C, Marie Hoogendam J, M. van der Veen F. FMRI, Antipsychotics and Schizophrenia. Influence of Different Antipsychotics on BOLD-Signal. Curr Pharm Des. 2010;16(18):2012-2025. doi:10.2174/138161210791293088. 


\section{Supplementary Information}
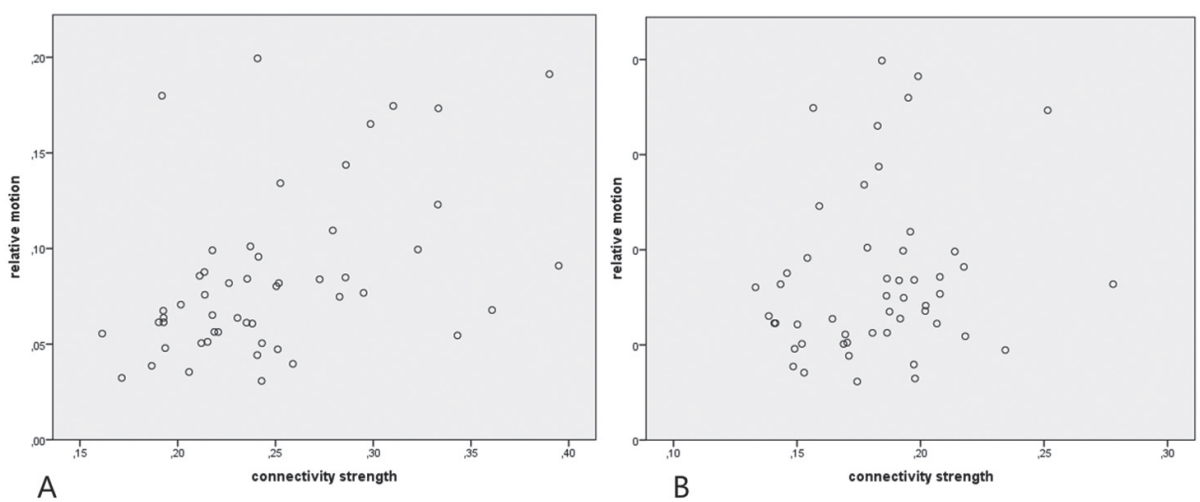

B

Figure S1 Correlation between relative motion and connectivity strength after ICA-AROMA (A) and spike regression (B) motion correction. (A) After ICA-AROMA motion correction a significant Pearson correlation was found between relative motion, i.e. framewise displacement, and connectivity strength, $r=0.452, P=0.001$. (B) After spike regression motion correction a non-significant Pearson correlation was found between relative motion, i.e. framewise displacement, and connectivity strength, $r=0.184, P=0.197$.
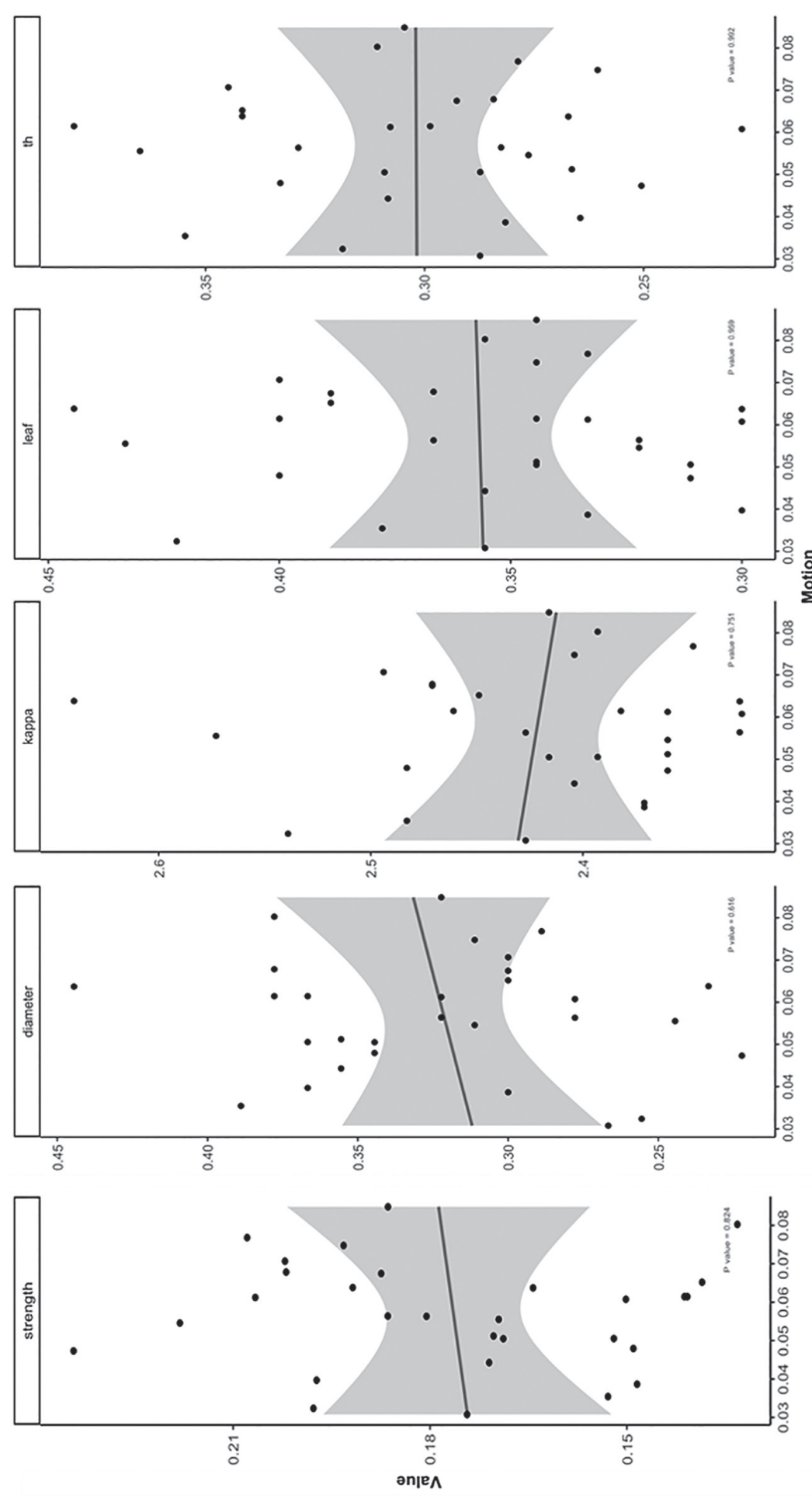
Table S1 Network outcomes of delirium patients, the post delirium patients and control subjects

Delirium ( $=9$ ) Post delirium Control $(N=13)$

$$
(\mathbf{N}=7)
$$

\begin{tabular}{llll}
\hline Strength & $0.17(0.03)$ & $0.16(0.01)^{\mathrm{a}, \mathrm{b}}$ & $0.19(0.02)$ \\
Diameter & $0.30(0.05)^{\mathrm{a}}$ & $0.30(0.06)$ & $0.28(0.04)$ \\
Kappa & $2.38(0.11)$ & $2.45(0.11)$ & $2.41(0.07)$ \\
Leaf fraction & $0.32(0.04)^{\mathrm{a}}$ & $0.36(0.04)$ & $0.35(0.03)$ \\
Tree hierarchy & $0.26(0.02)$ & $0.31(0.03)$ & $0.28(0.02)$ \\
\hline
\end{tabular}

Mean (SD) is shown. a Significantly different from control group. ${ }^{b}$ Significantly different from delirium group.

Table S2 Definitions of the additional network outcomes from the weighted network

\section{Outcome}

\section{Definition}

Clustering

The fraction of triangles around an individual node represents the clustering coefficient and is equivalent to the fraction of the node's neighbors that are also neighbors of each other.

Density The degree of an individual node is equal to the number of links connected to that node. The mean network degree is used as a measure of density.

Path length The average shortest path length between all pairs of nodes in the network.
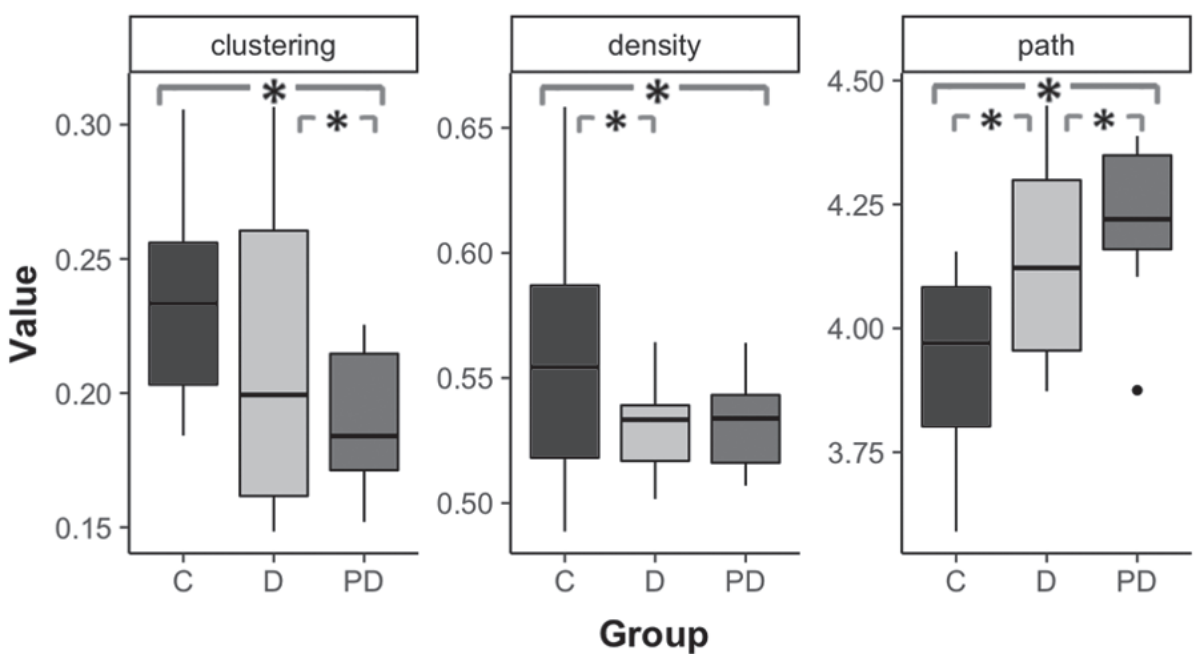

Figure S3 Boxplots of additional network outcomes from the weighted network, i.e. clustering coefficient, density and path length for the control, delirium and post delirium group. Path length was significantly increased during delirium compared to the control group with a difference of $0.21(95 \% \mathrm{Cl} 0.07-0.35$, corrected $\mathrm{P}=0.024)$. Path length was significantly increased after delirium compared to the control group with a difference of $0.32 \mathrm{~g} 95 \% \mathrm{Cl}$ $0.14-0.46$, corrected $\mathrm{P}=0.001$ ) and compared to the delirium group with a difference of $0.11(95 \% \mathrm{Cl}-0.08-0.27$, corrected $\mathrm{P}=0.021)$. Clustering was significantly decreased after delirium compared to the control group with a difference of $-0.05(95 \% \mathrm{Cl}-0.05-$ -0.02 , corrected $\mathrm{P}=0.001$ ) and compared to the delirium group with a difference of -0.04 $(95 \% \mathrm{Cl}-0.04--0.01$, corrected $\mathrm{P}=0.024)$. Density was significantly decreased during delirium compared to the control group with a difference of $-0.03(95 \% \mathrm{Cl}-0.06--0.01$ corrected $P=0.029)$. Density was significantly decreased during after delirium compared to the control group with a difference of $-0.03(95 \% \mathrm{Cl}-0.06--0.01$, corrected $\mathrm{P}=0.032)$. 

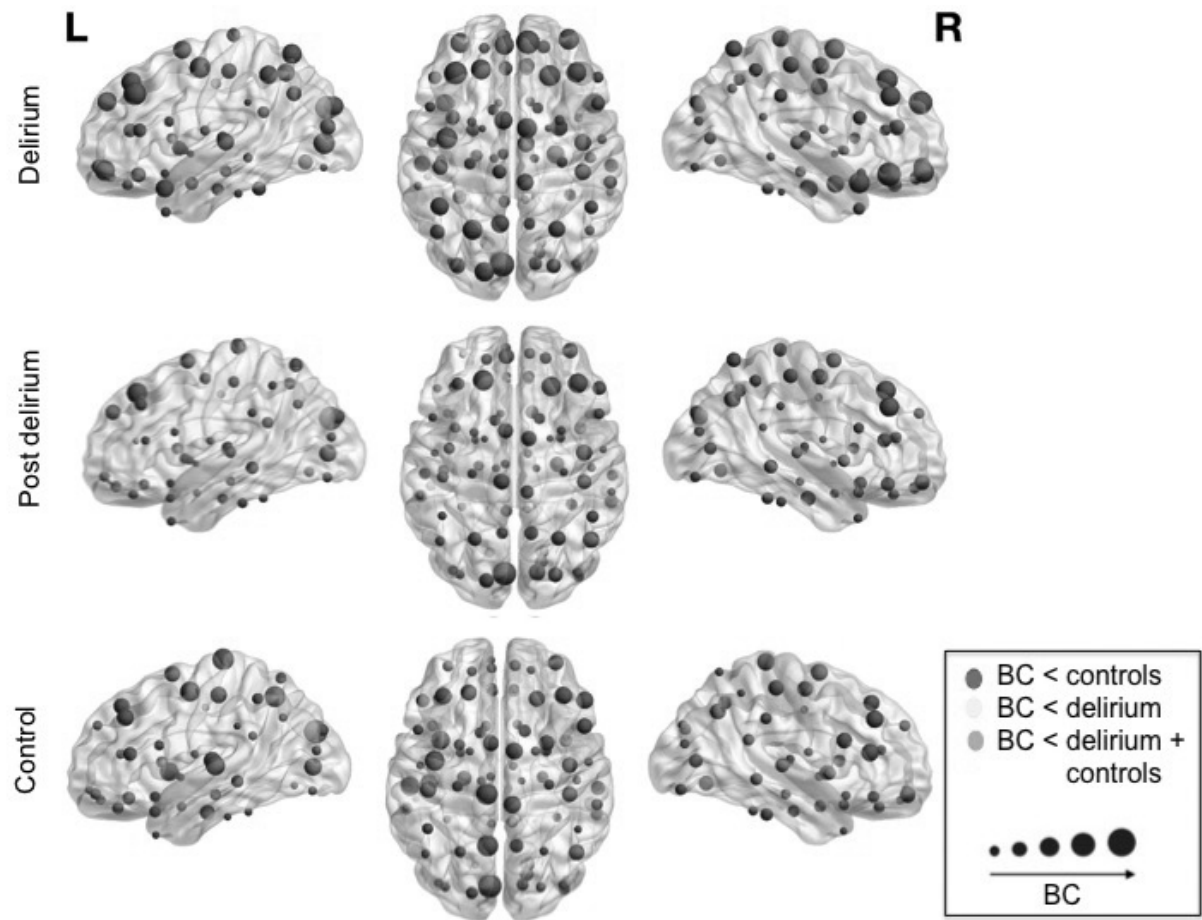

Figure S4 Visualization of the mean betweenness centrality (BC) in the MST backbone network of the delirium, post delirium and control group. The anatomical labeling atlas with 90 regions was used. The size of the nodes corresponds to the degree. Red, yellow and orange nodes mark regions with a significant group difference in degree. $\mathrm{BC}$ of the right inferior temporal gyrus was significantly lower in the delirium group compared to the healthy control group. BC of the left anterior cingulum and the right pallidum were significantly lower in the post delirium group compared to the healthy control group. BC of the orbital part of the right middle frontal gyrus, the right medial orbitofrontal cortex and the left anterior cingulum were lower in the post-delirium group compared to the delirium group. 


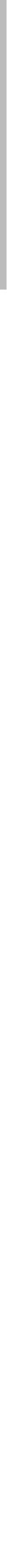




\section{Introduction}

Delirium is an acute disturbance of attention, awareness and cognition that tends to fluctuate over time. It is an acute and serious condition, affecting more than $15 \%$ of all hospitalized patients, and is related to poor outcomes such as prolonged length of hospital stay and long-term cognitive impairment. Although previous studies suggest multiple hypotheses towards underlying mechanisms, e.g. neurotransmitter imbalances, abnormal stress response and neuroinflammation, the exact pathogenesis of delirium remains poorly understood. Since adequate cognitive functioning requires interaction or functional connectivity between brain regions, delirium might constitute a clinical manifestation of the breakdown of functional network. Based on electroencephalography increased spectral variability and decreased complexity was found during delirium ${ }^{1}$. In a first functional magnetic resonance imaging ( $\mathrm{fMRI}$ ) study, activity of the dorsolateral prefrontal cortex and the posterior cingulate cortex (PCC) was positively correlated in delirium patients, whereas a negative correlation was found in controls without delirium ${ }^{2}$. In an additional study, whole-brain network analysis showed that delirious patients had reduced network integration and efficiency, which related to the duration and severity of the disorder ${ }^{3}$. Furthermore, loss of connectivity was found in the right PCC, usually a major hub (i.e., highly connected node) in the functional brain network. These results were all based on static functional brain networks. As delirium has a fluctuating course, it would be plausible that the disorder does not solely relies upon a static concept. Accordingly, the brain is a dynamic, flexible network that continuously reconfigures depending on the processes. Cognitive processes are thought to depend on this dynamical functioning or flexibility ${ }^{4}$. Decreased (hub) flexibility has been found to associate with decreased cognitive performance to a larger extent than static connectivity ${ }^{4}$ and delirium patients show acute cognitive disturbances. We therefore hypothesized that delirium patients have reduced flexibility of the PCC compared to clinical controls. Flexibility of the left and right PCC was assessed on four different levels, i.e. (1) the amplitude of the BOLD signal, (2) overall connectivity strength, (3) participation coefficient and (4) betweenness centrality (BC) based on the functional network.

\section{Methods}

Based on previously described data 2, 9 delirious patients and 13 clinical controls were included. The Institutional Review Board of Yonsei University approved the study and all participants gave informed consent. fMR preprocessing consisted of brain extraction, registration to a highresolution anatomical image, slice time correction, spatial smoothing, motion correction (i.e., subjects with a mean displacement $>0.2 \mathrm{~mm}$ were excluded) using MCFLIRT, additional spike regression, and bandpass filtering ${ }^{3}$. Average time series were extracted using the Brainnetome atlas (BNA) consisting of 210 cortical regions and 36 subcortical regions ${ }^{5}$. The functional connectivity matrices were obtained by calculation of the Pearson's correlation between all BNA regions and setting all negative values to zero.

Flexibility of the right and left PCC was assessed using the coefficient of variation (COV), which was calculated as the ratio between the standard deviation (SD) and mean of connection strength over all windows ${ }^{4}$. The window length was set to 25 samples (i.e., 62.5 seconds) and the shift was 5 samples (i.e., 12.5 seconds), resulting in 25 sliding-windows in each subject.

For each window the four measures were calculated. (1) The average of the amplitude the BOLD signal was calculated for each window. (2) Strength was defined as the average weight of all connections of the right and left PCC. The participation coefficient is the ratio between the number of modules the left or right PCC are connected to and the total number of modules in the network. (3) The participation coefficient was calculated based on binarized FC matrices with a sparsity range between $5 \%$ and $20 \%$. The area under the curve (AUC) over this sparsity range gave the final participation coefficient. (4) The BC was defined as the fraction of all shortest paths passing through the left or right PCC and was calculated based on the minimum spanning tree (MST) ${ }^{3}$. The MST is the backbone of the functional network containing the strongest connections without forming loops.

Using the Mann Whitney $U$ test, the difference in COV of these measures between delirium and clinical controls was assessed. P-values $<0.05$ were 
considered significant, no correction for multiple testing was performed due to the exploratory design of the study. Analyses were performed using the Brain Connectivity Toolbox and Matlab version R2012a (The MathWorks Incl., Natick, MA, USA).

\section{Results \& Discussion}

The delirious patients (age: 75.6 years (SD 6.9), male: $n=4$ (55.6\%)) were comparable to non-delirious clinical controls from the Databank for Brain Imaging at Gangnam Severance Hospital (age: 72.7 years (SD 6.7), male: 6 (46.2\%)) regarding age and gender ${ }^{2}$. No differences were found in average motion during $\mathrm{fMRI}(\mathrm{F}=1.35, \mathrm{p}=0.60)$. On average, the duration of delirium was 9 days (range 3 -19). The COV of the amplitude of the BOLD signal, strength, AUC of the participation coefficient and BC are presented in Figure 1, for delirious and clinical controls, separately for the left and right PCC. No differences were found between delirium patients and clinica controls.

In conclusion, flexibility of the left and right PCC based on four different measures was not significantly different in delirium patients compared to clinical controls, although previous work did show alterations in static connectivity in these patients. Therefore, disturbed flexibility might not be a correlate of the acute cognitive problems observed during delirium. However, the sample used in this study was small and the fMRI scans were relatively short, we therefore cannot draw firm conclusions. These results should be evaluated in a larger cohort with $\mathrm{fMRI}$ scans with a longer duration. Furthermore, not only the PCC should be studied to evaluate before disturbed flexibility can be excluded as an underlying mechanism of disturbed cognition during delirium
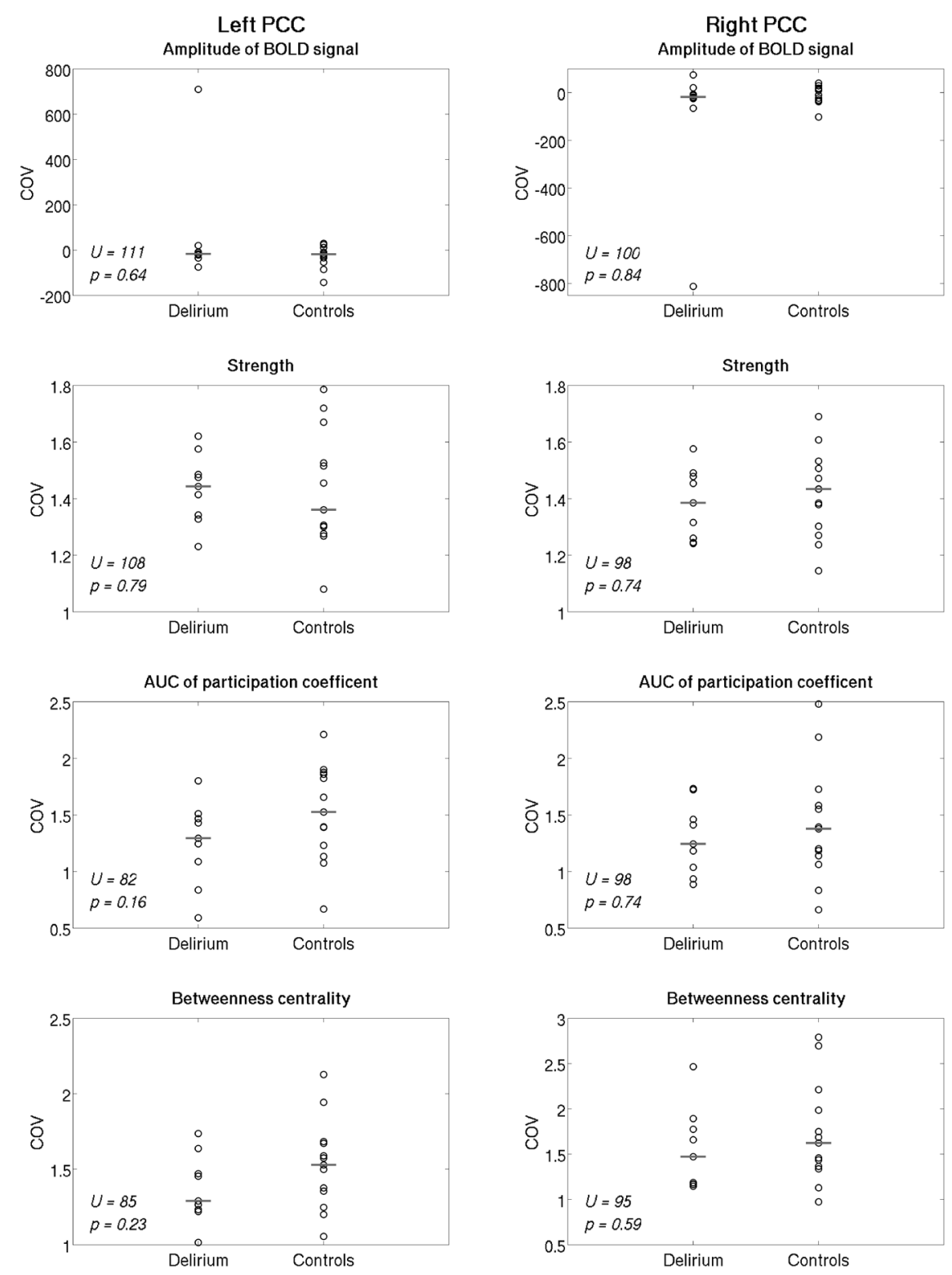

Figure 1 Coefficient of variation (COV) of the left and right posterior cingulate cortex (PCC) based on the amplitude of the BOLD signal, strength, AUC of participation coefficient and betweenness centrality (based on the minimum spanning tree) for delirium patients $(n=9)$ and control subjects $(n=13)$. Circles indicate individual values, red line is the median, $p$-values within the plots are based on the Mann Whitney $\mathrm{U}$ test. 


\section{Acknowledgements}

This work was supported by a grant from the Korea Health Technology R\&D Project through the Korea Health Industry Development Institute (KHIDI), funded by the Ministry of Health \& Welfare, Republic of Korea (grant number: HI16C0132).

\section{References}

1. van Dellen E, van der Kooi AW, Numan T, et al. Decreased Functional Connectivity and Disturbed Directionality of Information Flow in the Electroencephalography of Intensive Care Unit Patients with Delirium after Cardiac Surgery. Anesthesiology. 2014;121(2):328-335. doi:10.1097/ALN.0000000000000329

2. Choi S-H, Lee H, Chung T-S, et al. Neural Network Functional Connectivity During and After an Episode of Delirium. Am J Psychiatry. 2012;169(5):498-507. doi:10.1176/appi. ajp.2012.11060976

3. van Montfort SJT, van Dellen E, van den Bosch AMR, et al. Resting-state fMRI reveals network disintegration during delirium. Neurolmage Clin. 2018;20:35-41. doi:10.1016/J NICL.2018.06.024

4. Douw L, Wakeman DG, Tanaka N, Liu H, Stufflebeam SM. State-dependent variability of dynamic functional connectivity between frontoparietal and default networks relates to cognitive flexibility. Neuroscience. 2016;339:12-21. doi:10.1016/J. NEUROSCIENCE.2016.09.034

5. Fan L, Li H, Zhuo J, et al. The Human Brainnetome Atlas: A New Brain Atlas Based on Connectional Architecture. Cereb Cortex. 2016;26(8):3508-3526. doi:10.1093/cercor/ bhw157 


\section{Part 3}

Longitudinal changes after delirium 


\title{
Chapter 7
}

Functional brain network changes after major surgery and delirium

\author{
Simone JT van Montfort \\ Arjen JC Slooter \\ Ilse MJ Kant \\ Ellen Aarts \\ Lisette M Vernooij \\ Claudia D Spies \\ Jeroen Hendrikse \\ Edwin van Dellen
}

On behalf of the BioCog Consortium 


\section{Abstract}

Delirium, a clinical expression of acute encephalopathy, is associated with an increased risk of long-term cognitive impairment and dementia. Decreased global functional connectivity strength and a disturbed brain network organization have been described during postoperative delirium, and in patients with dementia. We hypothesized that longterm impact of postoperative delirium is reflected in functional brain network alterations after remission of the syndrome. We therefore studied whether postoperative delirium is associated with changes in the functional brain network over time in elderly patients undergoing elective surgery. Patients underwent clinical assessments and resting-state fMR before and three months after surgery. Delirium was assessed on the first seven postoperative days, using the Confusion Assessment Method for the Intensive Care Unit, the Nursing Delirium Screening Scale and chart review. After strict motion correction, $\mathrm{fMRI}$ global connectivity strength and network characteristics were calculated in 246 patients (35\% female, age 65-87 years), of whom 38 (16\%) developed postoperative delirium. Generalized linear mixed model analyses were performed to test if functional global connectivity strength, network efficiency and network integration differed between baseline and follow-up, and between patients who developed postoperative delirium (POD+) and patients who did not (POD-

). All models were adjusted for age, sex, pre-existing cognitive impairment, surgical specialty, surgery duration and center effects. Preoperatively, no differences in global functional connectivity strength, network efficiency, or network integration were found between POD+ and POD-. In the total study population, an increase in global functional connectivity strength over time was observed $(\beta=0.006, p=0.021)$. However, $P O D+$ showed a decrease in global functional connectivity strength over time $(\beta=-0.014, p=0.026)$ Patients who decreased in global functional connectivity strength (of whom $15.4 \% \mathrm{POD}+$ ) declined in trail making test $\mathrm{B}$ scores compared to the group that did not (of whom 10.3\% POD+) ( $\beta=11.04, p=0.034$ ). Postoperative delirium was not associated with changes in functional network efficiency or network integration over time. These findings indicate long-term impact of delirium on global functional connectivity strength after the syndrome clinically resolves, possibly related to lasting cognitive deterioration.

\section{Introduction}

Delirium, a clinical expression of acute encephalopathy, is characterized by an acute disturbance in attention and awareness, with additional cognitive deficits ${ }^{1,2}$. Delirium is by definition a consequence of one or more medical conditions and affects $15-25 \%$ of elderly patients after major elective surgery ${ }^{3}$, in that case called postoperative delirium. Delirium can be accompanied with patients' distress and increased length of admission, and is related to poor outcomes, such as long-term cognitive impairment and dementia ${ }^{3,4}$.

It has been hypothesized that delirium is a disconnection syndrome, reflecting a breakdown of functional brain networks ${ }^{5-8}$. The functional network is considered an abstraction of macroscale communication pathways between remote brain regions ${ }^{9}$. Functional connectivity maps can be characterized by the statistical interdependencies of time-series recorded from different brain areas, for example measured with functional magnetic resonance imaging (fMRI) ${ }^{10,11}$.

During delirium, the functional brain network can be characterized by decreased global functional connectivity strength, a less efficient and less integrated organization ${ }^{6,7,12,13}$. On a regional level, alterations in regional functional connectivity between parts of the default mode network (DMN, i.e. the posterior cingulate cortex, PCC) and the central executive network (CEN, i.e. the dorsolateral prefrontal cortex, DLPFC), were additionally shown during delirium ${ }^{14,15}$. These specific interactions are of interest for delirium, because awareness and attention disturbances are core symptoms. The DMN is implicated in wakeful rest and non-task states, and its activity is negatively correlated with the CEN, which is involved in selective attention processing ${ }^{16,17}$. Seven days after resolution of delirium, a decreased global functional connectivity strength has been observed, suggesting longterm impact of the disorder on the functional brain network (van Montfort et al., 2018). A decrease of global functional connectivity strength and disturbances in functional network efficiency and integration have also been reported in cognitive impairment and dementia ${ }^{18-26}$. 
A decrease of global functional connectivity strength due to delirium may relate to impaired outcomes, such as long-term cognitive impairment or dementia. However, up to now, studies evaluating delirium in relation to the brain network lacked baseline and follow-up measurements, i.e. fMRI measurements before the development of delirium, and after its resolution ${ }^{12,14,15}$. It is unknown whether delirium is related to a lasting change in global functional connectivity strength or brain network organization. The aim of this study was to test the hypothesis that postoperative delirium leads to a decrease in global functional connectivity strength, efficiency and integration of the functional brain network over time ${ }^{6,12,13}$. As a secondary analysis, the long-term effect of delirium on the regional connection between the PCC and the DLPFC was studied ${ }^{14,15}$

\section{Materials and methods}

\section{Study design and population}

This study is part of the Biomarker Development for Postoperative Cognitive Impairment in the Elderly (BioCog) project at the University Medical Center (UMC) Utrecht and Charité Hospital at Berlin (ethical approval numbers 14-469 respectively EA2/092/14 ) ${ }^{27}$. Inclusion criteria were European ancestry, age of 65 years or above, scheduled for a major elective surgery (i.e. orthopedic-, cardiac-, gastro-intestinal-, gynecological, urologic, maxillofacial- or otorhinolaryngologic surgery) of at least 60 minutes, an $\mathrm{fMRI}$ measurement at baseline and/or follow-up, and signed informed consent. Patients with one or more of the following characteristics were excluded: a life expectancy shorter than a year or evidence for (early) dementia as indicated with a score of 23 or lower on the Mini Mental State Examination (MMSE) ${ }^{28}$

\section{Procedures}

Included patients were invited to the hospital for a baseline measurement i.e. a clinical assessment and an MRI scan of the brain. The clinical assessment was administered by trained research staff. After surgery the patient group was followed in the hospital twice daily for delirium assessments until the day of discharge, with a maximum of seven postoperative days. Approximately three months after surgery, the patients were invited to the hospital again for the three month follow-up visit with similar measurements as during the baseline visit, i.e. a clinical assessment and an MRI scan of the brain.

\section{Clinical assessment}

Trial making test

At baseline and at follow-up, the trial making test (TMT) was administered We specifically focused on the TMT in this study, because decreased TMT test scores have previously been associated with delirium severity and also with decreased functional connectivity strength in dementia with Lewy bodies, a condition that is related to delirium ${ }^{19,29,30}$. Visual memory is required for TMT section $A$ and executive functioning are required for TMT section $B^{31-33}$

\section{Other characteristics}

Preoperative alcohol use was assessed using the self-reported Alcohol Use Disorders Identification Test (AUDIT) ${ }^{35,36}$. A cut-off value of 8 points was used to define alcohol misuse ${ }^{37}$. Preoperative depressive symptoms were estimated with the self-reported Geriatric Depression Scale (GDS) with 15 items ${ }^{38,39}$. A score of 6 was used as cut-off to define depression. Preoperative functional impairment was measured with the Barthe Index 40-42. Preoperative physical status was scored by anesthesiologists (in training) using the validated American Society of Anesthesiologists (ASA) classification ${ }^{43-45}$. The diagnoses of preoperative hypertension and diabetes were extracted from the medical records of the patients. Preoperative transient ischemic attack (TIA) or stroke was determined using the medical records of the patients and scores from neuroradiologists on cortical, subcortical and lacunar infarcts, based on the STRIVE criteria ${ }^{46}$, as previously described by Kant and colleagues ${ }^{47}$.

\section{Delirium assessment}

Delirium was defined according to the 5 th edition of the Diagnostic and Statistical Manual of Mental Disorders (DSM-5) criteria ${ }^{1}$. The Confusion Assessment Method for the Intensive Care Unit (CAM-ICU) ${ }^{48}$, the Nursing Delirium Screening Scale (Nu-DESC) ${ }^{49}$ and chart review ${ }^{50}$ were used to assess delirium by trained research staff. Patients were considered delirious in case of 2 or more cumulative points on the Nu-DESC and/or a 
positive CAM-ICU score and/or a chart review that showed descriptions of delirium (e.g., confused, agitated, drowsy, disorientated, delirious, receiving delirium-related antipsychotic therapy). In case of uncertainty, a delirium expert was consulted to make the final decision on the diagnosis of the patient. If a patient was delirious, we additionally registered the duration of delirium (in days).

\section{Image processing}

MRI scans

Imaging was performed on a 3T Achieva (Philips Medical Systems, Best, the Netherlands) scanner in Utrecht and on a 3T TrioTim (Siemens Healthineers, Erlangen, Germany) scanner in Berlin. A T1-weighted 3D Turbo Field Echo (TFE) structural image was made in Utrecht or a T1-weighted Magnetization Prepared Rapid Gradient Echo (MPRAGE) structural image was made in Berlin. Sequence parameters of the T1 TFE were: TR $=7.9 \mathrm{~ms}$, TE $=4.5$ $\mathrm{ms}$, flip angle $=8^{\circ}, 192$ sagittal slices, voxel size $1 \times 1 \times 1 \mathrm{~mm}$. Sequence parameters of the T1 MPRAGE were: TR $=2500 \mathrm{~ms}$, TE $=4.77 \mathrm{~ms}$, flip angle $=7^{\circ}, 192$ sagittal slices, voxel size $1 \times 1 \times 1 \mathrm{~mm}$. A T2*-weighted gradientecho - echoplanar imaging (GE-EPI) image was used for the resting-state blood-oxygen-level dependent (BOLD) fMRI ( $r$-fMRI) scan, which had the following sequence parameters: $\mathrm{TR}=2000 \mathrm{~ms}, \mathrm{TE}=30 \mathrm{~ms}$, flip angle $=78^{\circ}$, 32 transversal slices, voxel size $3 \times 3 \times 3,75 \mathrm{~mm}, 238$ volumes in 7 minutes and 55 seconds. The rs-fMRI was made in a dark room and patients were asked to close their eyes and to stay awake.

\section{Preprocessing}

Preprocessing of the images was performed using the FMRIB's Software Library (FSL) ${ }^{51-53}$. The brain was automatically extracted from the T1weighted scan ${ }^{54}$. The time series were corrected for motion with MCFLIRT 55,56. Motion during $\mathrm{fMRI}$ measurements can induce bias, therefore additional motion correction is necessary ${ }^{57-61}$. Volumes that exceeded the threshold of $0.2 \mathrm{~mm}$ framewise displacement ${ }^{62}$ were removed and a regression analysis with 36 motion components was done. The motion components were: three voxel-wise displacement parameters and their white matter, cerebrospinal fluid, global time courses, and the quadrates, temporal derivatives and quadrates of the derivatives of these six parameters ${ }^{60}$. The average time series from the cerebral spinal fluid, the white matter and grey matter intensities were defined after tissue segmentation with the FMRIB's Automated Segmentation Tool (FAST) ${ }^{63}$. A band-pass filter $(0.01-0.08 \mathrm{~Hz})$ was applied ${ }^{60}$.

The functional scan was registered to the high-resolution anatomical image using rigid registration. The anatomical scan was subsequently registered to the Montreal Neurological Institute (MNI) 152 T1-weighted 2 mm image in standard space with affine registration. Functional scans were slice-time corrected and spatially smoothed to reduce noise $(5 \mathrm{~mm}$ full-width-halfmaximum). To ensure stabilized magnetization, the first 15 volumes were deleted. If the remaining data was less than 240 seconds, the patient was excluded from further analysis ${ }^{64}$.

Connectivity and network analysis

We selected 264 putative functional areas that cover all cortical and subcortical brain regions ${ }^{65}$. All connectivity and network calculations were performed in MATLAB, version R2016b, using publicly available and personalized scripts. To estimate regional mean time series, voxel time series within each region were averaged. Using Pearson's correlations, functional connectivity was subsequently calculated between all time series pairs, resulting in a $264 \times 264$ functional connectivity matrix for each patient.

Minimum spanning tree (MST) network backbones were extracted using Kruskal's algorithm ${ }^{66}$. The MST connections were all based on positive correlation values, thus avoiding the problematic interpretation of negative BOLD correlations ${ }^{67,68}$. The MST can be considered as the backbone of the original network, connecting all regions without forming loops ${ }^{66-68}$, which allows a relatively unbiased comparison with another network with the same number of regions and connections ${ }^{67-69}$. Correlation values of the connectivity matrix were ranked and the highest value was included as the first MST connection using Kruskal's algorithm ${ }^{66}$. The second highest value was then added as an MST connection, etcetera until all 264 regions were connected. If adding a connection would result in a loop or triangle, this connection was discarded and the next value was evaluated. 
Formally, a maximum spanning tree was thus constructed; the highest connectivity values were used to construct the MST as these connections were expected to reflect communication with minimal cost. To be consistent with previous literature using this approach, we refer to the minimum spanning tree or MST throughout this manuscript.

During delirium, altered global functional network connectivity strength, network efficiency, network organization and altered regional connectivity between the PCC and the DLPFC have been shown, therefore these outcomes were investigated in this study ${ }^{6,12-15}$

Global functional connectivity strength

For each patient, global functional connectivity strength was calculated by averaging the connectivity values of all connections in the MST. This approach was chosen to maximize the signal-to-noise ratio of included connections within a standardized network backbone that is expected to reflect major information processing routes, to avoid problematic interpretation of negative BOLD correlations, and methodological bias of spurious connections or arbitrary thresholding in group comparisons of network topology $67,68,70,71$

Network efficiency (MST diameter)

To assess network efficiency, the MST diameter was used. The MST diameter describes the number of edges connecting the most remote nodes in the MST and therefore gives an indication of the efficiency of global network organization ${ }^{67,68}$. A low MST diameter indicates efficient information processing between remote brain regions ${ }^{67,68,72}$

Network integration (MST leaffraction)

To estimate the network integration, the MST leaf fraction was used. The MST leaf fraction describes the proportion of regions with a degree of one, i.e. regions that are connected to only one other region ${ }^{67,68}$. A large MST leaf fraction indicates an integrated network topology $67,68,72$.
Functional connectivity between the PCC and the DLPFC (PCC-DLPFC-FC) The PCC was defined as the region centered at coordinates $(M N I x / y / z)$ : -11/-56/16 (Power atlas region \#77), -3/-49/13 (Power atlas region \#78) and 11/-54/17 (Power atlas region \#82) ${ }^{14,15,65}$. The DLPFC left was defined as the region centered at coordinates ( $\mathrm{MNI} \times / \mathrm{y} / \mathrm{z}$ ): $-42 / 38 / 21$ (Power atlas region \#167) and -34/55/4 (Power atlas region \#176) 14,15,65. The DLPFC right was defined as the region centered at coordinates ( $M N I x / y / z): 38 / 43 / 15$ (Power atlas region \#168) and 40/18/40 (Power atlas region \#175) 14,15,65 The connection between the PCC and the left or the right DLPFC was calculated for each patient using Pearson's correlations between the mean time series of the regions.

\section{Statistical analysis}

Statistical analyses were performed in $\mathrm{R}$ statistics (Version 3.5.1). Baseline characteristics were compared between patients who developed postoperative delirium and patients that did not, using the Chi-square test for categorical variables and independent samples t-test or MannWhitney U-test for continuous variables as appropriate. Surgical specialty was categorized in cardiothoracic, intra-abdominal, orthopedic and other. Surgery duration was analyzed per minute and duration of delirium in days.

We conducted generalized linear mixed models to analyze the longitudinal effect of global functional connectivity strength, MST diameter, MST leaf fraction and PCC-DLPFC-FC derived from the baseline and follow-up fMRI measurements in the same patients. Patients with only one measurement available were also included in the model ${ }^{73}$. We compared baseline measures of global functional connectivity strength, MST leaf fraction, and MST diameter as well as PCC-DLPFC-FC between patients that developed postoperative delirium and patients that did not. We further studied whether these measures changed over time. Univariate analyses were performed with time and delirium in the fixed part and 'patient' as random part. To assess whether global functional connectivity strength, MST leaf fraction, and MST diameter varied over time among patients who developed postoperative delirium and patients that did not, an interaction term was added between delirium and time in the fixed part of the model. Thereafter, we conducted multivariable analyses by adding a priori selected covariates 
to the fixed part of the model. These included age, sex, MMSE, surgical specialty, surgery duration and center. Selection of confounders was made based on clinical reasoning and knowledge obtained from literature 3,74 . Models were compared based on the Akaike's information criterion (AIC). Restricted maximum likelihood estimation (REML) was used to generate unbiased variance estimates for the final models. Estimates are expressed as linear regression coefficients $(\beta)$ with $95 \%$ confidence intervals $(95 \% \mathrm{Cl})$.

As exploratory analysis, we performed Spearman's correlation analyses to investigate the association between the MST measures (global functiona connectivity strength, MST diameter and MST leaf fraction) and duration of the delirium (in patients that developed postoperative delirium only). Change in TMT score (A or B) was compared between the delirium and the non-delirium group using a generalized linear mixed model. Furthermore, if a significant change was found between the delirium and the non-delirium group in an $\mathrm{fMRl}$ outcome measure over time, we additionally evaluated this change in relation to the change in cognitive performance as measured with TMT scores. The group that decreased on the fMRI outcome measure was compared with regard to the TMT change over time to the group that increased or remained stable on the $\mathrm{fMRI}$ outcome, using a generalized linear mixed model. A p-value below 0.05 was considered statistically significant.

\section{Results}

Demographics

In this study, the total eligible cohort consisted of 554 patients that performed baseline measurements. In total, 246 patients with sufficient quality of the pre- and or postoperative fMRI scan and available postoperative data, were included in this study (Supplementary Information Figure S1). The included patients were generally more often from center Utrecht, younger, had less comorbidities and scored higher on the TMT B than excluded patients (Supplementary Information Table S1). Of the 246 included patients, 38 (16\%) developed delirium within the first seven postoperative days. Patients who developed delirium were generally older, had a longer duration of surgery and had a longer hospital stay (Table 1).
fMRI data of sufficient quality was available for 216 patients at baseline and for 160 patients at three months follow-up. Of these patients, 130 had fMR data of sufficient quality available at both measurements (Supplementary Information Table S2). Patients that had two fMRI scans of sufficient quality available, were generally more often from center Utrecht, younger and had a better performance on TMT B.

The evaluated $\mathrm{fMRI}$ outcomes appeared stable over the used timespan, tested in a group of non-surgical controls (Supplementary Information part I, and Table S4). 


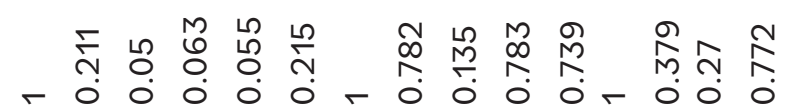

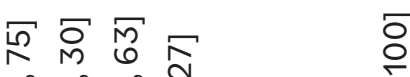

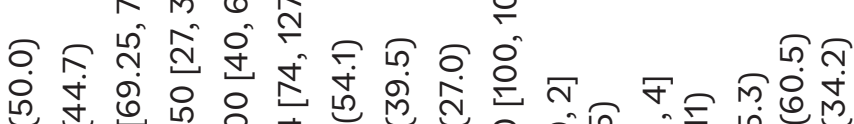

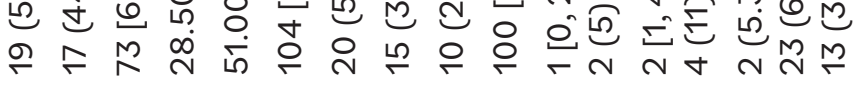

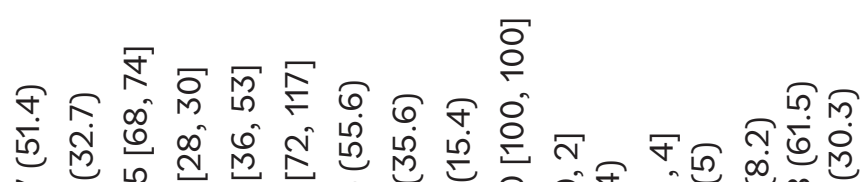

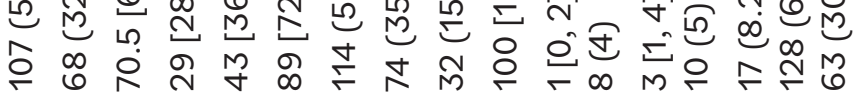

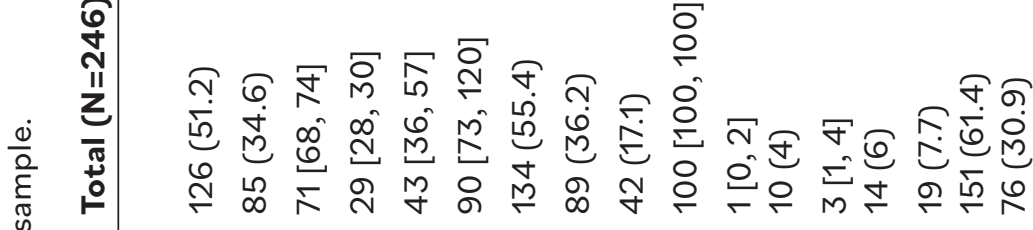

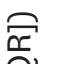

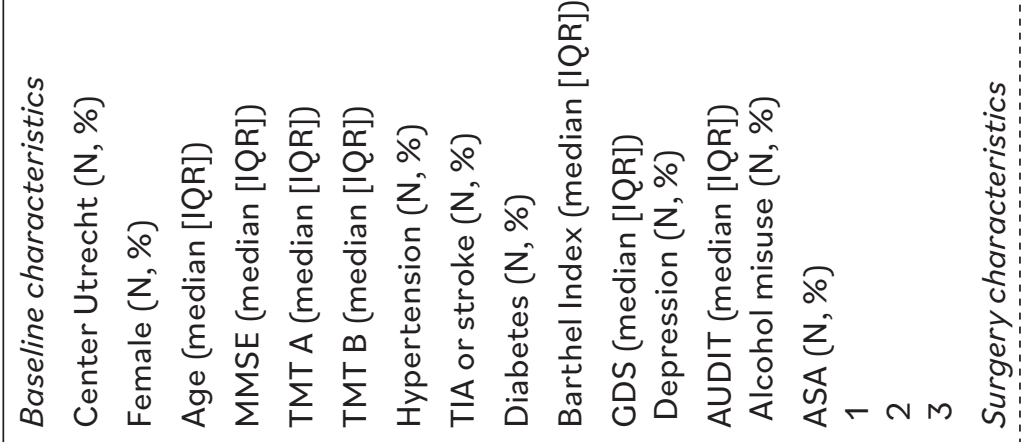

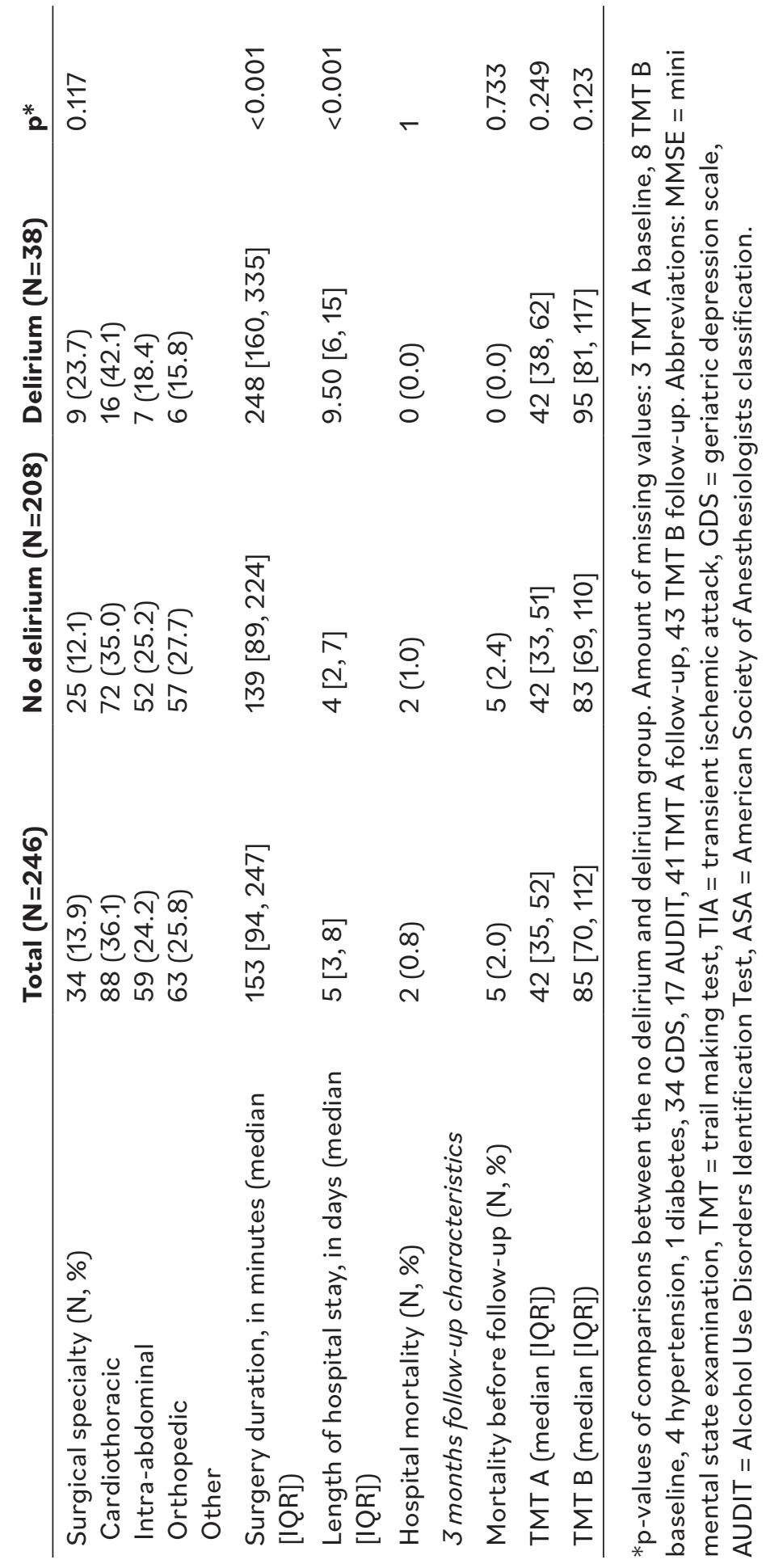


TMT scores over time

TMT values at baseline and follow-up are described in Table 1. The delirium and the non-delirium group did not differ in change of TMT scores over time (TMT A ( $\beta=0.625, p=0.835)$ and TMT $B(\beta=-0.427, p=0.944)$.

Global functional connectivity strength

Patients who developed postoperative delirium did not differ from patients who remained delirium-free regarding global functional connectivity strength at baseline

$(\beta=0.006, \mathrm{Cl}=-0.003-0.016, p=0.165)$ (for mean values, see Supplementary Information Table S3). Over time, global functiona connectivity strength increased in the total study population $(\beta=0.006$, $\mathrm{Cl}=0.000-0.012, \mathrm{p}=0.021)$. However, a decline in global functional connectivity strength was found for patients who developed delirium, relative to an increase in global functional connectivity strength in patients who remained delirium-free $(\beta=-0.014, \mathrm{Cl}=-0.028--0.008, p=0.026)$ (Figure 1). No significant correlations were found between duration of delirium and global functional connectivity strength at three months ( $\rho=-0.067, p=0.787$ ), or change in global functional connectivity strength $(\rho=-0.051, p=0.852)$.

\section{Global functional connectivity strength \& change in TMT B scores}

A group of 130 patients had sufficient quality $\mathrm{fMRI}$ scans available at both baseline and follow-up. In this group, 52 patients (40\%) showed a decrease in global functional connectivity strength over time. Patients with decreased global functional connectivity strength at follow-up (with $15.4 \%$ prevalence of postoperative delirium) had significantly declined in TMT B score $(\beta=11.04, p=0.034)$ compared to patients with equal or increased global functional connectivity strength at follow-up (10.3\% prevalence of postoperative delirium) (Figure 2). For individual trajectories of the delirium patient on global functional connectivity strength and TMT B scores, see Supplementary Information Figure S2.
Functional network efficiency (MST diameter)

No differences were found between delirium and non-delirium patients on MST diameter at baseline $(\beta=0.004, \mathrm{Cl}=-0.004-0.012, \mathrm{p}=0.339$ ), (see Supplementary Information Table S3). Over time, no significant change was found in MST diameter for the total study population $(\beta=0.000, \mathrm{Cl}=-0.004$ - 0.005, $p=0.871)$. In addition, we did not find differences in MST diameter for delirium and non-delirium patients over time $(\beta=0.000, \mathrm{Cl}=-0.013-$ $0.013, p=0.976$ ) (Supplementary Information Figure S3). No significant correlations were found between delirium duration and MST diameter at three months follow-up ( $\rho=0.029, p=0.907)$ or change in MST diameter $(\rho=0.234, p=0.383)$.

\section{Functional network integration (MST leaf fraction)}

No differences were found between delirium and non-delirium patients on MST leaf fraction at baseline $(\beta=0.000, \mathrm{Cl}=-0.009-0.008, p=0.843)$, (see Supplementary Information Table S3). Over time, no significant change was found in MST leaf fraction for the total study population $(\beta=-0.003$, $\mathrm{Cl}=-0.008-0.001, p=0.134)$. In addition, we did not find differences in MST leaf fraction for delirium and non-delirium patients over time $(\beta=0.002$, $\mathrm{Cl}=-0.001--0.001, \mathrm{p}=0.771$ ) (Supplementary Information Figure S3). No significant correlations were found between delirium duration and MST leaf fraction at three months follow-up ( $\rho=-0.036, p=0.883)$ or change in MST leaf fraction $(\rho=-0.179, p=0.506)$.

\section{PCC-DLPFC-FC}

No differences were found between delirium and non-delirium patients on PCC-DLPFC-FC at baseline (left: $\beta=-0.018, C l=-0.059-0.024, p=0.397$, right: $\beta=-0.001, \mathrm{Cl}=-0.044-0.043, \mathrm{p}=0.979$ ), (see Supplementary Information Table S3). Over time group, no significant change was found in PCC-DLPFC-FC for the total study population (left: $\beta=0.022, \mathrm{Cl}=-0.002$ - 0.047, $p=0.007$, right: $\beta=-0.005, C l=-0.029-0.019, p=0.669)$. In addition, we did not find differences in PCC-DLPFC-FC for delirium and nondelirium patients over time (left: $\beta=0.010, \mathrm{Cl}=-0.002-0.047, p=0.757$, right: $\beta=0.002, \mathrm{Cl}=-0.029-0.019, p=0.858$ ) (Supplementary Information Figure S3). 


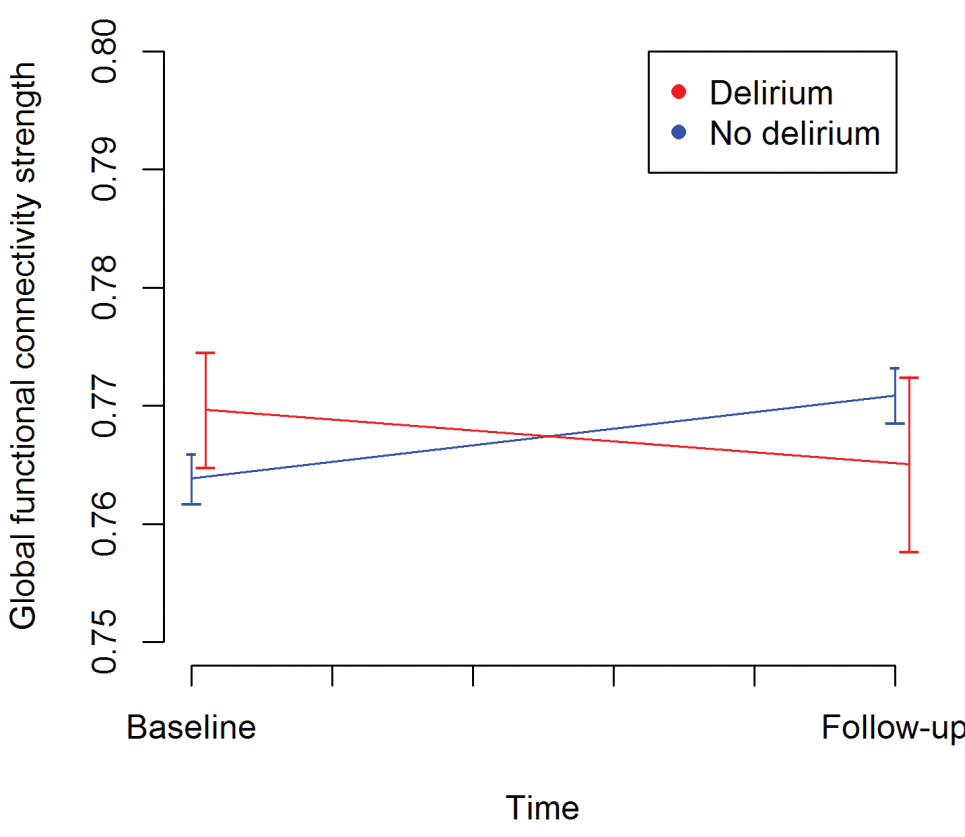

Figure $1 \mathrm{Global}$ functional connectivity strength over time for the delirium and non-delirium group. Global functional connectivity strength was evaluated at baseline (preoperatively) and at three months follow-up, in an elderly population undergoing major, elective surgery Global functional connectivity strength significantly decreased in patients that developed postoperative delirium, but increased in patients that did not develop postoperative delirium

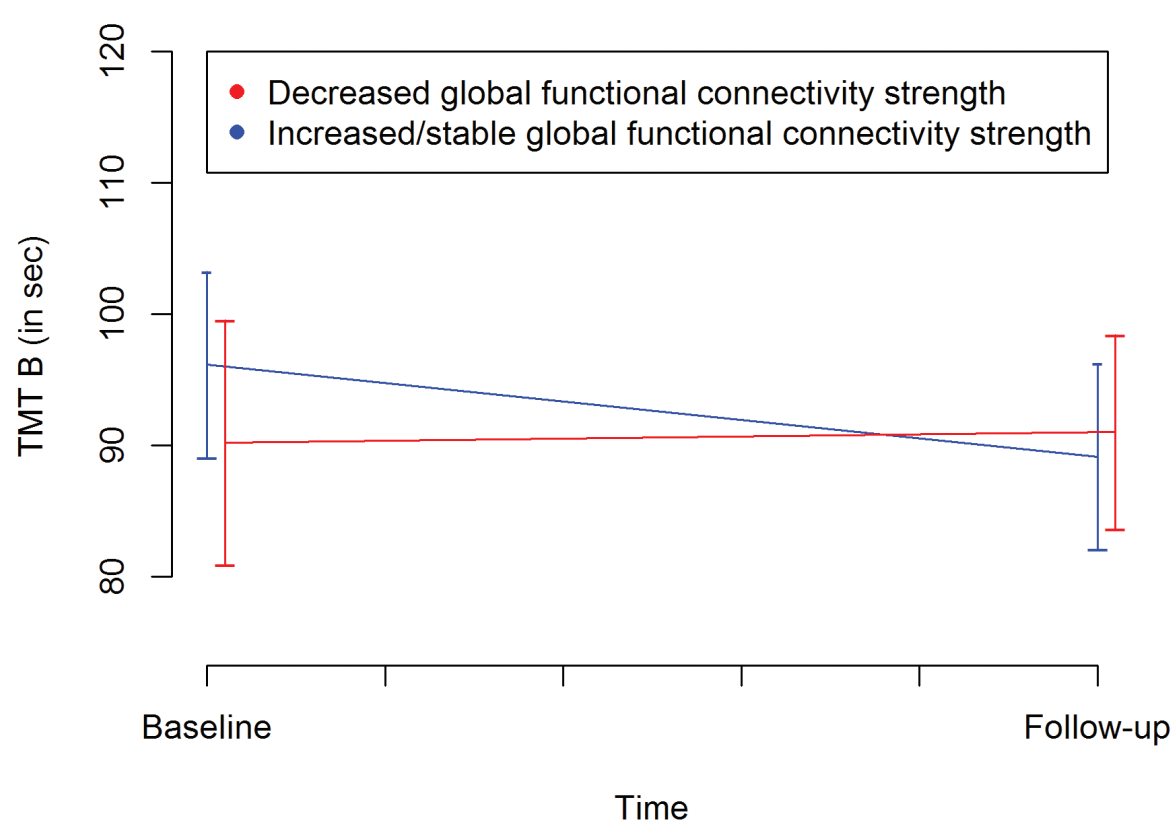

Figure 2 Trail making test B scores over time for the group that decreased in global functional connectivity strength and the group that did increased or remained equal in global functional connectivity strength. Trail making test B score is a reaction time, the lower the score, the better the performance. The group that decreased in global functional connectivity strength significantly declined in trail making test B performance. Normally, a learning effect occurs when the task is repeated, which can be observed in the group with an equal or increased global functional connectivity strength.

\section{Discussion}

In this longitudinal study, we investigated the impact of major surgery and postoperative delirium on $\mathrm{fMRI}$ functional brain networks in an elderly population. Three months after surgery and relative to the preoperative measurements, global functional connectivity strength was decreased in patients who had suffered postoperative delirium, but increased in patients who remained delirium-free. Patients who showed decreased global functional connectivity strength at follow-up had a decline in executive function compared to patients with an increased global functional connectivity strength. This study is the first to empirically evaluate the 
changes in the functional brain network, over a time period of three months, in relation to the presence or absence of postoperative delirium, and to link these changes to postoperative cognitive performance.

No baseline differences in functional brain networks were found between patients who developed delirium postoperatively and those who remained delirium-free, which is in line with previous studies showing that functional brain network characteristics that are altered during delirium do not necessarily reflect for the syndrome ${ }^{14,15,83,84}$. The onset of delirium seems therefore to reflect new functional network impairments. However, the dynamic nature of the functional brain network, as well as the complex interaction between brain network structure and function, might imply that other network characteristics predispose patients to delirium than those observed during the syndrome itself ${ }^{7}$.

In a previous study, we found that global functional connectivity strength was lower in patients one week after delirium compared to healthy controls 12, but no fMRI data were available before the onset of delirium in that investigation. The findings presented in the current study show that the decrease of global functional connectivity strength is even found three months after remission of the syndrome. The decrease was associated with deteriorated executive function, as measured with the TMT B. As decreased global functional connectivity strength is also observed in patients with severe cognitive impairment and dementia ${ }^{18-26}$, we speculate that the lasting decrease in global functional connectivity strength could be related to poor cognitive outcomes in some patients after recovery from delirium, such as long-term cognitive impairment. As we found no decline in TMT B test scores at follow-up between patients with and without postoperative delirium, we could not test if delirium-related cognitive decline was mediated by decreased global functional connectivity strength.

The absence of long-term cognitive impairment in the delirium group may be explained by several factors. It could be that the patients that had more severe cognitive problems were lost to follow-up, as they were unable to come to the hospital for the follow-up measurements. Furthermore, although delirium is a risk factor for long-term cognitive impairment, not all patients will develop long-term cognitive problems. We may have included a relatively healthy group of elective surgery patients, due to an extensive study protocol ${ }^{27}$ and strict motion correction. This group may, in general, have had higher cognitive reserves and strong brain plasticity, possibly resulting in the ability to cognitively recover from delirium ${ }^{75,76}$.

An interesting observation is that major surgery was associated with an increase in global functional connectivity strength over time. However, it is known that surgery alone may also increase the risk of long-term cognitive dysfunction ${ }^{77,78}$. The interpretation of general alterations in connectivity strength is not straightforward. A simulation study of activity-dependent neural degeneration indicated that increased functional connectivity strength may reflect high neural activity levels, which may result in neural damage and could therefore be considered as an early state of vulnerability ${ }^{79}$. As major surgery may result in neuroinflammation and reduced oxygen supply, the increase of global functional connectivity strength in the majority of patients without postoperative delirium may be a reflection of the resilient brain response to the complex interaction of these (and possibly other) factors ${ }^{79-82}$.

At three months follow-up, we found that postoperative delirium was not associated with changes in functional network efficiency, functional network integration or regional functional connectivity between the posterior cingulate cortex and the dorsolateral prefrontal cortex. This finding is consistent with the previously mentioned study on functional brain networks that showed that seven days after delirium was clinically resolved, there were no lasting changes in functional network efficiency or network integration ${ }^{12}$. Alterations in functional network efficiency and functional network integration seem thus to be related to the clinical syndrome of delirium and may recover when delirium resolves.

An important strength of this study is that we were able to obtain measurements before the onset of delirium by studying surgical patients preoperatively. In addition, robust methods were used and a large number of patients was included in this multicenter study. However, there are also important limitations. Due to rigorous motion correction, a considerable 
part of our study population had to be excluded, which may have resulted in selection of patients who are less vulnerable for delirium. Furthermore, we specifically focused on the TMT in this study, because decreased TMT test scores have previously been associated with delirium severity and also with decreased global functional connectivity strength in dementia with Lewy bodies ${ }^{19,29,30}$. However, long-term cognitive impairment after delirium based on functional network changes may additionally be reflected in other cognitive tasks. Another limitation is that information on medication use of the patients was not stored. We can therefore not exclude possible drugrelated effects on the $\mathrm{fMRI}$ measurements. Nevertheless, all patients were non-hospitalized during the $\mathrm{fMRI}$ measurements.

\section{Conclusion}

Delirium seems to result in a long-term decrease in global functional connectivity strength. Decreased global functional connectivity strength was associated with cognitive decline, irrespective of postoperative delirium. We speculate that global functional connectivity strength is related to the increased risk of long-term cognitive impairment and dementia after delirium. Notably, due to the exclusion of patients with fMRI scans of insufficient quality, which were generally older, had more comorbidities and scored worse on the TMT B, our results could have been influenced by selection effects. Still, our study may provide new insights into the biological substrate of long-term brain changes after surgery and delirium.

\section{Acknowledgements}

This work was supported by funding from the European Union Seventh Framework Program under Grant Agreement No. 602461 [FP7/20072013] and funding from the Dutch Organization for Health Research and Development (ZonMW) under Grant Agreement No. 60-63600-98-711. We would like to especially thank Noam Rubin, Raoul Lieben and Rutger van de Leur for the technical support with the $\mathrm{fMRI}$ analyses and Fienke Ditzel, Jacqueline Vromen and Wietze Pasma for the support with data management.

\section{References}

1. American Psychiatric Association. Diagnostic and Statistical Manual of Mental Disorders (5th Ed.). Washington, DC; 2013.

2. Slooter A, Otte W, Devlin J, et al. Updated nomenclature of delirium and acute encephalopathy. Statements endorsed by the American Academy of Neurology (AAN) American Delirium Society (ADS), European Academy of Neurology (EAN), European Delirium Association (EDA), European Geriatric Medici. Intensive Care Med. 2020.

3. Marcantonio ER. Delirium in Hospitalized Older Adults. Solomon CG, ed. N EnglJ Med. 2017;377(15):1456-1466. doi:10.1056/NEJMcp1605501

4. O'Malley G, Leonard M, Meagher D, O'Keeffe ST. The delirium experience: A review.J Psychosom Res. 2008;65(3):223-228. doi:10.1016/J.JPSYCHORES.2008.05.017

5. Sanders RD. Hypothesis for the pathophysiology of delirium: Role of baseline brain network connectivity and changes in inhibitory tone. Med Hypotheses. 2011;77(1):140143. doi:10.1016/j.mehy.2011.03.048

6. van Dellen E, van der Kooi AW, Numan T, et al. Decreased Functional Connectivity and Disturbed Directionality of Information Flow in the Electroencephalography of Intensive Care Unit Patients with Delirium after Cardiac Surgery. Anesthesiology. 2014;121(2):328-335. doi:10.1097/ALN.0000000000000329

7. van Montfort SJT, van Dellen E, Stam CJ, et al. Brain network disintegration as a fina common pathway for delirium: a systematic review and qualitative meta-analysis. Neurolmage Clin. 2019;23. doi:10.1016/j.nicl.2019.101809

8. Young JWS. The network model of delirium. Med Hypotheses. 2017;104:80-85 doi:10.1016/j.mehy.2017.05.027

9. Bassett DS, Sporns O. Network neuroscience. Nat Neurosci. 2017;20(3):353-364 doi:10.1038/nn.4502

10. Aertsen AM, Gerstein GL, Habib MK, Palm G. Dynamics of neuronal firing correlation modulation of "effective connectivity." J Neurophysiol. 1989;61(5):900-917. doi:10.1152/ jn.1989.61.5.900

11. van den Heuvel MP, Hulshoff Pol HE. Exploring the brain network: a review on restingstate fMRI functional connectivity. Eur Neuropsychopharmacol. 2010;20(8):519-534. doi:10.1016/j.euroneuro.2010.03.008

12. van Montfort SJT, van Dellen E, van den Bosch AMR, et al. Resting-state fMRI reveals network disintegration during delirium. Neurolmage Clin. 2018;20:35-41. doi:10.1016/J. NICL.2018.06.024 
13. Numan T, Slooter AJC, van der Kooi AW, et al. Functional connectivity and network analysis during hypoactive delirium and recovery from anesthesia. Clin Neurophysiol. 2017;128(6):914-924. doi:10.1016/j.clinph.2017.02.022

14. Choi SH, Lee H, Chung TS, et al. Neural network functional connectivity during and after an episode of delirium. Am J Psychiatry. 2012;169(5):498-507. doi:10.1176/appi. ajp.2012.11060976

15. OhJ, Shin JE, Yang KH, et al. Cortical and subcortical changes in resting-state functional connectivity before and during an episode of postoperative delirium. Aust New ZealJ Psychiatry. 2019;53(8):794-806. doi:10.1177/0004867419848826

16. Raichle ME. The Brain's Default Mode Network. Annu Rev Neurosci. 2015;38(1):433 447. doi:10.1146/annurev-neuro-071013-014030

17. Fox MD, Corbetta M, Snyder AZ, VincentJL, Raichle ME. Spontaneous neuronal activity distinguishes human dorsal and ventral attention systems. Proc Natl Acad Sci U S A. 2006;103(26):10046-10051. doi:10.1073/pnas.0604187103

18. Carlos G, Stam CJ, Gómez C, et al. Disturbed Beta Band Functional Connectivity in PatientsWith Mild Cognitive Impairment: An MEG Study. IEEE Trans Biomed Eng. 2009;56(6):1683-1690. doi:10.1109/TBME.2009.2018454

19. van Dellen E, de Waal H, van der Flier WM, et al. Loss of EEGNetwork Efficiency Is Related to Cognitive Impairment in Dementia With Lewy Bodies. Mov Disord 2015;30(13):1785-1793. doi:10.1002/mds.26309

20. Filippi M, Basaia S, Canu E, et al. Brain network connectivity differs in early-onset neurodegenerative dementia. Neurology. 2017;89(17):1764-1772. doi:10.1212/ WNL.0000000000004577

21. Peraza LR, Taylor J-P, Kaiser M. Divergent brain functional network alterations in dementia with Lewy bodies and Alzheimer's disease. Neurobio/ Aging. 2015;36(9):24582467. doi:10.1016/j.neurobiolaging.2015.05.015

22. Minati L, Chan D, Mastropasqua $\mathrm{C}$, et al. Widespread alterations in functional brain network architecture in amnestic mild cognitive impairment. J Alzheimers Dis. 2014;40(1):213-220. doi:10.3233/jad-131766

23. Chang T-Y, Huang K-L, Ho M-Y, et al. Graph theoretical analysis of functional networks and its relationship to cognitive decline in patients with carotid stenosis.J Cereb Blood Flow Metab. 2016;36(4):808-818. doi:10.1177/0271678X15608390

24. Zeng K, Wang Y, Ouyang G, Bian Z, Wang L, Li X. Complex network analysis of resting state EEG in amnestic mild cognitive impairment patients with type 2 diabetes. Front Comput Neurosci. 2015;9:133. doi:10.3389/fncom.2015.00133
25. Frantzidis CA, Vivas AB, Tsolaki A, Klados MA, Tsolaki M, Bamidis PD. Functional disorganization of small-world brain networks in mild Alzheimer's disease and amnestic Mild cognitive impairment: An EEG study using Relative Wavelet Entropy (RWE). Front Aging Neurosci. 2014;6:224. doi:10.3389/fnagi.2014.00224

26. Koenig T, Prichep L, Dierks T, et al. Decreased EEG synchronization in Alzheimer's disease and mild cognitive impairment. Neurobiol Aging. 2005;26(2):165-171. doi:10.1016/j.neurobiolaging.2004.03.008

27. Winterer $\mathrm{G}$, Androsova $\mathrm{G}$, Bender $\mathrm{O}$, et al. Personalized risk prediction of postoperative cognitive impairment - rationale for the EU-funded BioCog project. Eur Psychiatry 2018. doi:10.1016/j.eurpsy.2017.10.004

28. Folstein M, Anthony JC, Parhad I, Duffy B, Gruenberg EM. The meaning of cognitive impairment in the elderly. J Am Geriatr Soc. 1985;33(4):228-235. http://www.ncbi. nlm.nih.gov/pubmed/3989183. Accessed January 28, 2019

29. Casey CP, Lindroth $\mathrm{H}$, Mohanty $\mathrm{R}$, et al. Postoperative delirium is associated with increased plasma neurofilament light. Brain. 2020;143(1):47-54. doi:10.1093/brain/ awz354

30. Lindroth H, Bratzke L, Twadell S, et al. Predicting postoperative delirium severity in older adults: The role of surgical risk and executive function. Int J Geriatr Psychiatry. 2019;34(7):1018-1028. doi:10.1002/gps.5104

31. Lezak M, Howieson D, Loring D, Fischer J. Neuropsychological assessment. 2004 https://books.google.com/

ooks?hl=nl\&Ir=\&id=FroDVkVKA2EC\&oi=fnd\&pg=PA3\&dq=Lezak+MD, + Howieson $+D$ B,+Loring+DW.+Neuropsychological+Assessment.+4th+ed.+New+York:+Oxford+University+Press\%3B+2004.+\&ots=q60i_MOq5M\&sig=II9AE2frUA0BX6gKZboKUfbL0Q4. Accessed March 18, 2020.

32. Reitan RM. The relation of the Trail Making Test to organic brain damage.J Consult Psychol. 1955;19(5):393-394. doi:10.1037/h0044509

33. Arbuthnott K, Frank J. Trail Making Test, Part B as a Measure of Executive Control: Validation Using a Set-Switching Paradigm.J Clin Exp Neuropsychol. 2000;22(4):518528. doi:10.1076/1380-3395(200008)22:4;1-0;FT518

34. Bowie CR, Harvey PD. Administration and interpretation of the Trail Making Test. Nat Protoc. 2006;1(5):2277-2281. doi:10.1038/nprot.2006.390

35. Bohn MJ, Babor TF, Kranzler HR. The Alcohol Use Disorders Identification Test (AUDIT): validation of a screening instrument for use in medical settings. J Stud Alcohol. 1995;56(4):423-432. doi:10.15288/jsa.1995.56.423 
36. Reinert DF, Allen JP. The Alcohol Use Disorders Identification Test: An Update of Research Findings. Alcohol Clin Exp Res. 2007;31(2):185-199. doi:10.1111/j.15300277.2006.00295.x

37. Babor T, Higgins-Biddle J, Saunders J. AUDIT: the alcohol use disorders identification test: guidelines for use in primary health care. 2001. http://apps.who.int/iris/ bitstream/handle/10665/67205/WHO_MSD_?sequence=1. Accessed January 28, 2019

38. Yesavage JA, Brink TL, Rose TL, et al. Development and validation of a geriatric depression screening scale: A preliminary report. J Psychiatr Res. 1982;17(1):37-49. doi:10.1016/0022-3956(82)90033-4

39. Yesavage JA, Sheikh JI. Geriatric Depression Scale (GDS): Recent evidence and development of a shorter version. Clin Gerontol. 1986;5(1-2):165-173. doi:10.1300/ j018v05n01_09

40. Lübke N, Meinck M, Von Renteln-Kruse W. [The Barthel Index in geriatrics. A context analysis for the Hamburg Classification Manual]. Z Gerontol Geriatr. 2004;37(4):316326. doi:10.1007/s00391-004-0233-2

41. Collin C, Wade DT, Davies S, Horne V. The Barthel ADL Index: a reliability study. Int Disabil Stud. 1988;10(2):61-63. http://www.ncbi.nlm.nih.gov/pubmed/3403500. Accessed January 28, 2019.

42. Mahoney FI, Barthel DW. FUNCTIONAL EVALUATION: THE BARTHEL INDEX. Md State Med J. 1965;14:61-65. http://www.ncbi.nlm.nih.gov/pubmed/14258950. Accessed January 28, 2019.

43. Aronson WL, McAuliffe MS, Miller K. Variability in the American Society of Anesthesiologists Physical Status Classification Scale. AANA J. 2003;71(4):265-274. http://www.ncbi.nlm.nih.gov/pubmed/13677221. Accessed January 28, 2019.

44. Sankar A, Johnson SR, Beattie WS, Tait G, Wijeysundera DN. Reliability of the American Society of Anesthesiologists physical status scale in clinical practice. BrJ Anaesth. 2014;113(3):424-432. doi:10.1093/bja/aeu100

45. Owens WD, Felts JA, Spitznagel EL. ASA physical status classifications: a study of consistency of ratings. Anesthesiology. 1978;49(4):239-243. http://www.ncbi.nlm. nih.gov/pubmed/697077. Accessed January 28, 2019.

46. Wardlaw JM, Smith EE, Biessels GJ, et al. Neuroimaging standards for research into small vessel disease and its contribution to ageing and neurodegeneration. Lancet Neurol. 2013;12(8):822-838. doi:10.1016/S1474-4422(13)70124-8

47. Kant IMJ, Mutsaerts HJMM, van Montfort SJT, et al. The association between frailty and MRI features of cerebral small vessel disease. Sci Rep. 2019;9(1):11343. doi:10.1038/ s41598-019-47731-2
48. Ely EW, Margolin R, Francis J, et al. Evaluation of delirium in critically ill patients: Validation of the Confusion Assessment Method for the Intensive Care Unit (CAMICU). Crit Care Med. 2001;29(7).

49. Gaudreau J-D, Gagnon P, Harel F, Tremblay A, Roy M-A. Fast, Systematic, and Continuous Delirium Assessment in Hospitalized Patients: The Nursing Delirium Screening Scale. J Pain Symptom Manage. 2005;29(4):368-375. doi:10.1016/J PAINSYMMAN.2004.07.009

50. Inouye SK, Leo-summers ÃL, Zhang Y, Bogardus ST, Leslie DL, Agostini J V. A ChartBased Method for Identification of Delirium : Validation Assessment Method. Chart. 2005:312-318.

51. Jenkinson M, Beckmann CF, Behrens TEJ, Woolrich MW, Smith SM. FSL. Neuroimage. 2012;62(2):782-790. doi:10.1016/j.neuroimage.2011.09.015

52. Smith $S M$, Jenkinson $M$, Woolrich MW, et al. Advances in functional and structural MR image analysis and implementation as FSL. In: Neurolmage. Vol 23. ; 2004:S208-S219. doi:10.1016/j.neuroimage.2004.07.051

53. Woolrich MW, Jbabdi S, Patenaude B, et al. Bayesian analysis of neuroimaging data in FSL. Neuroimage. 2009;45(1):S173-S186. doi:10.1016/j.neuroimage.2008.10.055

54. Smith SM. Fast robust automated brain extraction. Hum Brain Mapp. 2002;17(3):143 155. doi:10.1002/hbm.10062

55. Jenkinson M, Bannister P, Brady M, Smith S. Improved Optimization for the Robust and Accurate Linear Registration and Motion Correction of Brain Images. Neuroimage. 2002;17(2):825-841. doi:10.1006/nimg.2002.1132

56. Jenkinson M, Smith S. A global optimisation method for robust affine registration of brain images. Med Image Anal. 2001;5(2):143-156. doi:10.1016/S1361-8415(01)00036-

57. Power JD, Barnes KA, Snyder AZ, Schlaggar BL, Petersen SE. Spurious but systematic correlations in functional connectivity MRI networks arise from subject motion. Neuroimage. 2012;59(3):2142-2154. doi:10.1016/j.neuroimage.2011.10.018

58. Power JD, Schlaggar BL, Petersen SE. Recent progress and outstanding issued in motion correction resting state fmri. Neuroimage. 2015;105:536-551. doi:10.1016/j neuroimage.2014.10.044.Recent

59. Pruim RHR, Mennes M, Buitelaar JK, Beckmann CF. Evaluation of ICA-AROMA and alternative strategies for motion artifact removal in resting state fMRI. Neuroimage. 2015;112:278-287. doi:10.1016/j.neuroimage.2015.02.063 
60. Satterthwaite TD, Elliott MA, Gerraty RT, et al. An improved framework for confound regression and filtering for control of motion artifact in the preprocessing of restingstate functional connectivity data. Neuroimage. 2013;64:240-256. doi:10.1016/j. neuroimage.2012.08.052

61. Ciric R, Wolf DH, Power JD, et al. Benchmarking of participant-level confound regression strategies for the control of motion artifact in studies of functional connectivity. Neuroimage. 2017;154:174-187. doi:10.1016/j.neuroimage.2017.03.020

62. Power JD, Mitra A, Laumann TO, Snyder AZ, Schlaggar BL, Petersen SE. Methods to detect, characterize, and remove motion artifact in resting state fMRI. Neuroimage. 2014;84:320-341. doi:10.1016/j.neuroimage.2013.08.048

63. Zhang Y, Brady M, Smith S. Segmentation of brain MR images through a hidden Markov random field model and the expectation-maximization algorithm. IEEE Trans Med Imaging. 2001;20(1):45-57. doi:10.1109/42.906424

64. Birn, R.M., Molloy, E.K., Patriat, R., Parker, T., Meier, T.B., Kirk, G.R., Nair, V.A., Meyerand, M.E., Prabhakaran V. The effect of scan length on the reliability of resting state fMRI connectivity estimates. Neuroimage. 2013;83:550-558. doi:10.1016/J NEUROIMAGE.2013.05.099

65. Power JD, Cohen AL, Nelson SM, et al. Functional network organization of the human brain. Neuron. 2011;72(4):665-678. doi:10.1016/j.neuron.2011.09.006

66. Kruskal JB. On the Shortest Spanning Subtree of a Graph and the Traveling Salesman Problem. Proc Am Math Soc. 1956;7(1):48-50. https://www.jstor.org/stable/ pdf/2033241.pdf. Accessed March 20, 2019

67. Stam CJ, Tewarie P, Van Dellen E, van Straaten ECW, Hillebrand A, Van Mieghem P. The trees and the forest: Characterization of complex brain networks with minimum spanning trees. Int J Psychophysiol. 2014;92(3):129-138. doi:10.1016/j. ijpsycho.2014.04.001

68. Tewarie P, van Dellen E, Hillebrand A, Stam CJ. The minimum spanning tree: An unbiased method for brain network analysis. Neuroimage. 2015;104:177-188. doi:10.1016/j. neuroimage.2014.10.015

69. van Diessen E, Numan T, van Dellen E, et al. Opportunities and methodological challenges in EEG and MEG resting state functional brain network research. Clin Neurophysiol. 2015;126(8):1468-1481. doi:10.1016/j.clinph.2014.11.018

70. Fornito A, Zalesky A, Breakspear M. Graph analysis of the human connectome: Promise, progress, and pitfalls. Neuroimage. 2013;80:426-444. doi:10.1016/j. neuroimage.2013.04.087
71. van Wijk BCM, Stam CJ, Daffertshofer A, Stam C, Apkarian A. Comparing Brain Networks of Different Size and Connectivity Density Using Graph Theory. Sporns O, ed. PLoS One. 2010;5(10):e13701. doi:10.1371/journal.pone.0013701

72. van Dellen E, Bohlken MM, Draaisma L, et al. Structural Brain Network Disturbances in the Psychosis Spectrum. Schizophr Bull. 2016;42(3):782-789. doi:10.1093/schbul/ sbv178

73. Peters SAE, Bots ML, Den Ruijter HM, et al. Multiple imputation of missing repeated outcome measurements did not add to linear mixed-effects models. / Clin Epidemiol. 2012;65(6):686-695. doi:10.1016/j.jclinepi.2011.11.012

74. Inouye SK, Westendorp RG, Saczynski JS. Delirium in elderly people. Lancet. 2014;383(9920):911-922. doi:10.1016/S0140-6736(13)60688-1

75. Monk TG, Price CC. Postoperative cognitive disorders. Curr Opin Crit Care. 2011;17(4):376-381. doi:10.1097/MCC.0b013e328348bece

76. Fick DM, Kolanowski A, Beattie E, McCrow J. Delirium in early-stage alzheimer's disease: enhancing cognitive reserve as a possible preventive measure. / Geronto/ Nurs. 2009;35(3):30-38. doi:10.3928/00989134-20090301-06

77. Evered LA, Silbert BS. Postoperative Cognitive Dysfunction and Noncardiac Surgery. Anesth Analg. 2018;127(2):496-505. doi:10.1213/ANE.0000000000003514

78. Needham MJ, Webb CE, Bryden DC. Postoperative cognitive dysfunction and dementia: what we need to know and do. BrJ Anaesth. 2017;119(suppl_1):i115-i125. doi:10.1093/ bja/aex354

79. de Haan W, Mott K, van Straaten ECW, Scheltens P, Stam CJ. Activity dependent degeneration explains hub vulnerability in Alzheimer's disease. PLoS Comput Biol. 2012;8(8):e1002582. doi:10.1371/journal.pcbi.1002582

80. Slater JP, Guarino T, Stack J, et al. Cerebral Oxygen Desaturation Predicts Cognitive Decline and Longer Hospital Stay After Cardiac Surgery. Ann Thorac Surg. 2009;87(1):36-45. doi:10.1016/J.ATHORACSUR.2008.08.070

81. van Harten AE, Scheeren TWL, Absalom AR. A review of postoperative cognitive dysfunction and neuroinflammation associated with cardiac surgery and anaesthesia. Anaesthesia. 2012;67(3):280-293. doi:10.1111/j.1365-2044.2011.07008.x

82. Lee H, Mashour GA, Noh G-J, Kim S, Lee U. Reconfiguration of network hub structure after propofol-induced unconsciousness. Anesthesiology. 2013;119(6):1347-1359. doi:10.1097/ALN.0b013e3182a8ec8c

83. van Montfort SJT, Dellen E, Wattel LL, et al. Predisposition for delirium and EEC characteristics. Clin Neurophysiol. February 2020. doi:10.1016/J.CLINPH.2020.01.023

84. Kyeong S, Shin JE, Yang KH, Lee WS, Chung T-S, Kim J-J. Neural predisposing factor of postoperative delirium in elderly patients with femoral neck fracture. Sci Rep. 2018;8(1):7602. doi:10.1038/s41598-018-26030-2 

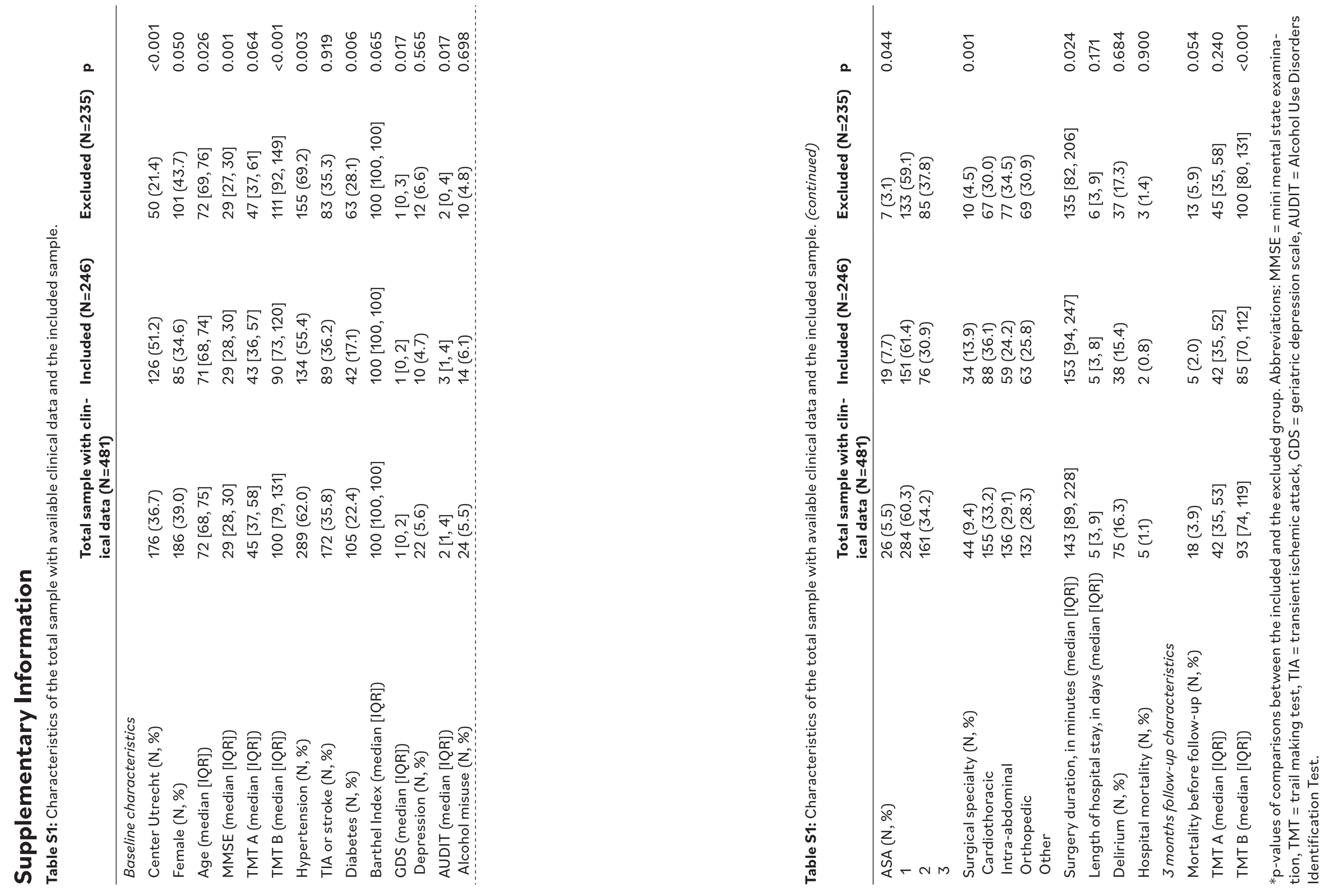

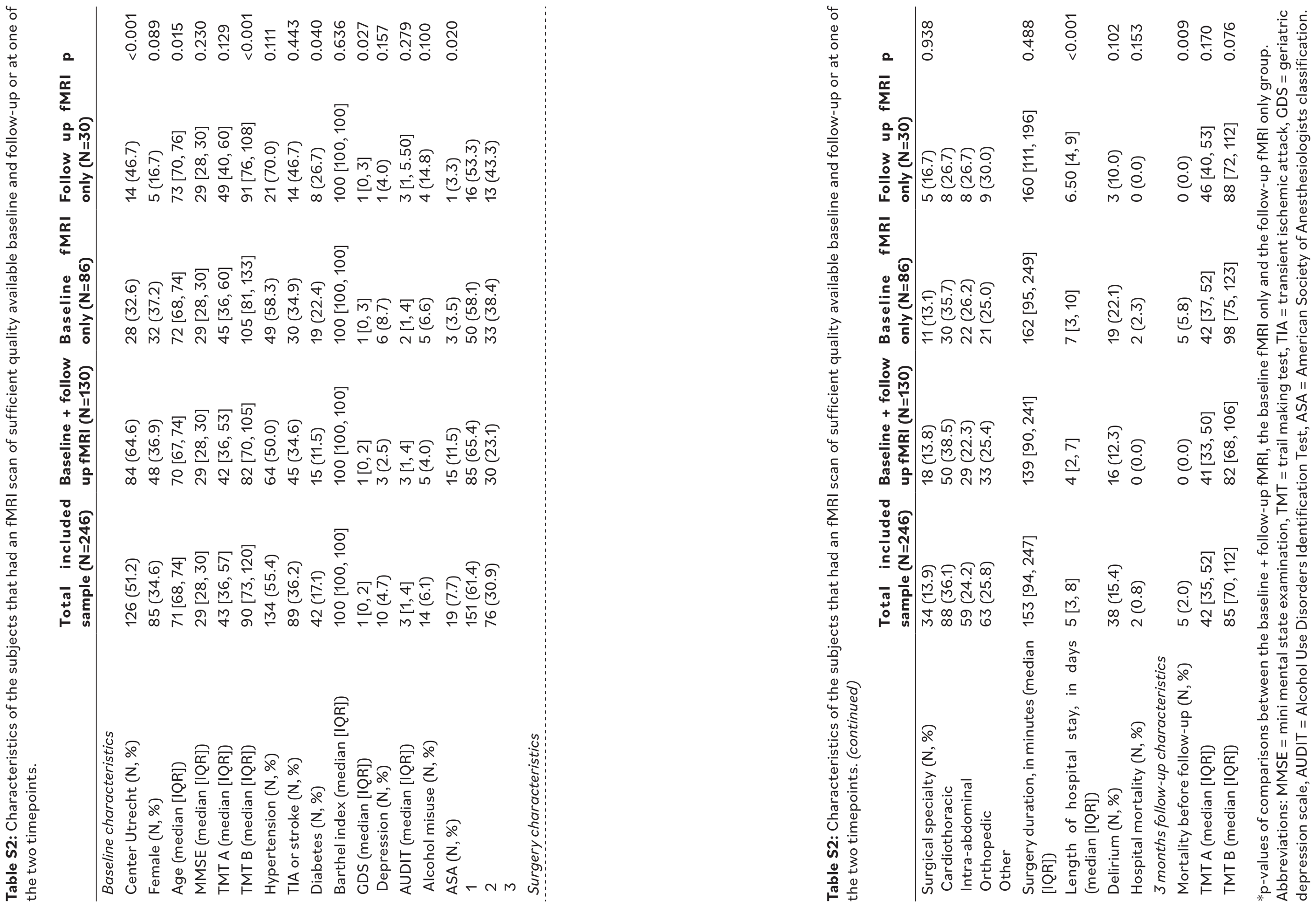
Table S3: Mean values of the functional brain network outcomes at baseline and follow up for the delirium and the no delirium group.

\begin{tabular}{lll} 
Functional brain network outcome & No delirium & Delirium \\
\hline Global functional connectivity strength baseline & 0.76 & 0.77 \\
(mean \pm SD) & \pm 0.03 & \pm 0.03 \\
Global functional connectivity strength follow up & 0.77 & 0.77 \\
(mean \pm SD) & \pm 0.03 & \pm 0.03 \\
& & \\
MST diameter baseline (mean \pm SD) & 0.13 & 0.14 \\
& \pm 0.02 & \pm 0.03 \\
MST diameter follow up (mean \pm SD) & 0.13 & 0.14 \\
& \pm 0.02 & \pm 0.02 \\
& & \\
MST leaf fraction baseline (mean \pm SD) & 0.42 & 0.41 \\
& \pm 0.02 & \pm 0.02 \\
MST leaf fraction follow up (mean $\pm S D$ ) & 0.42 & 0.41 \\
& \pm 0.02 & \pm 0.02 \\
Functional connectivity PCC - left DLPFC baseline & 0.25 & \\
(mean $\pm S D$ ) & \pm 0.10 & \pm 0.11 \\
Functional connectivity PCC - left DLPFC follow up & 0.27 & 0.25 \\
(mean $\pm S D$ ) & \pm 0.12 & \pm 0.11 \\
& & \\
Functional connectivity PCC - right DLPFC baseline & 0.26 & 0.25 \\
(mean $\pm S D$ ) & \pm 0.11 & \pm 0.12 \\
Functional connectivity PCC - right DLPFC follow up & 0.25 & 0.26 \\
(mean $\pm S D$ ) & \pm 0.11 & \pm 0.15 \\
\hline
\end{tabular}

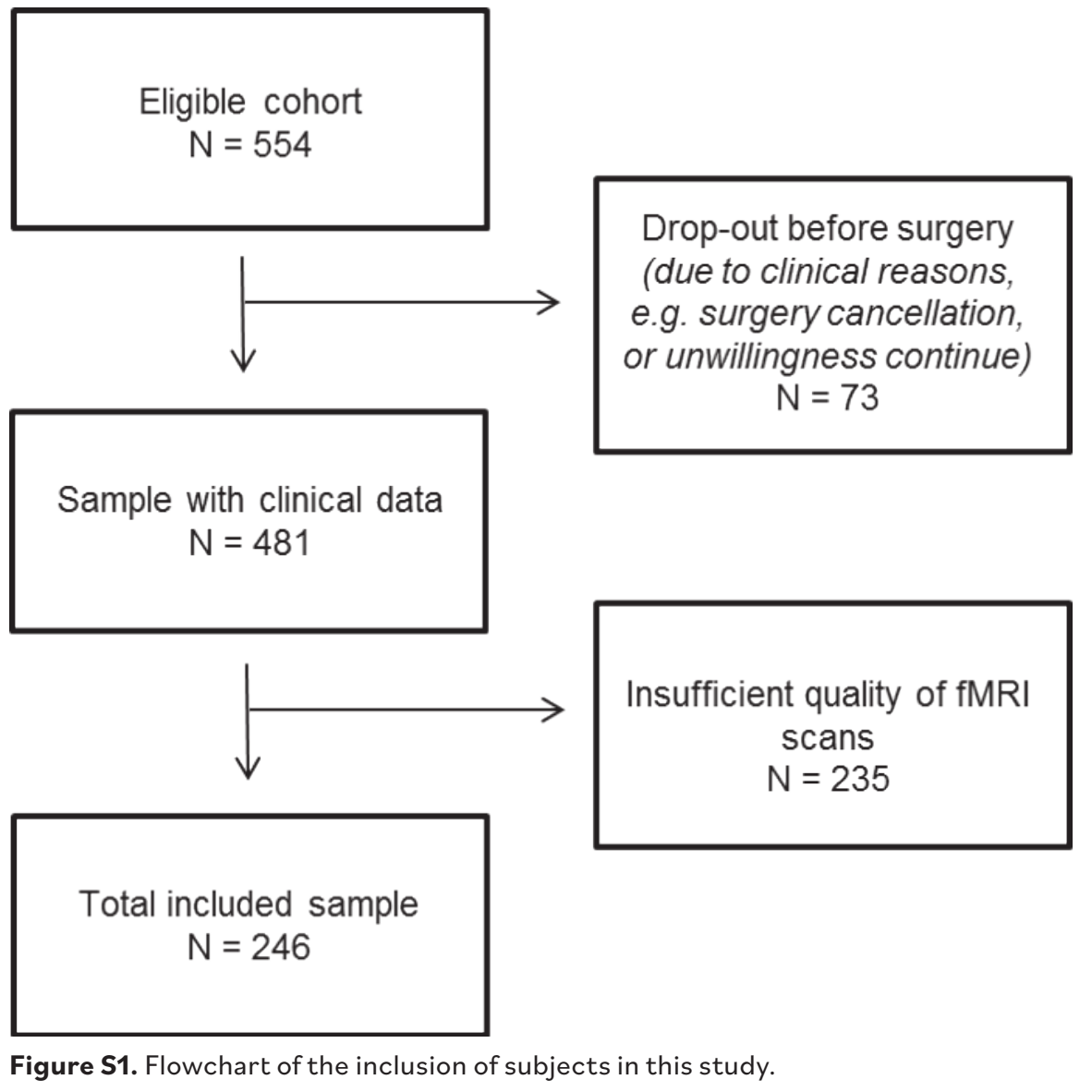

Supplementary Information Part I. Consistency of fMRI measurements over time

To test consistency of the $\mathrm{fMRI}$ outcomes, changes in global functional connectivity strength, MST diameter and MST leaf fraction were analyzed within a non-surgical control group. The non-surgical control group was recruited via general practitioners in Utrecht and in Berlin (for demographic see Supplementary Information Table S4). Inclusion criteria of non-surgical control participants were similar as the surgical group, with the exception of scheduled major surgery in the coming three months. A mixed model was constructed comparing the baseline and the follow-up measurement.

Consistency analysis in the non-hospitalized control group revealed no differences between baseline and follow-up of global functional connectivity 
strength (mean \pm SD baseline $=0.77 \pm 0.03$, mean \pm SD follow-up $=0.77$ $\pm 0.03, \beta=0.007, p=0.175$ ), MST diameter (mean \pm SD baseline $=0.13$ \pm 0.02 , mean \pm SD follow-up $=0.13 \pm 0.02, \beta=-0.002, p=0.631$ ), MST leaf fraction (mean \pm SD baseline $=0.41 \pm 0.02$, mean \pm SD follow-up $=0.42 \pm 0.02$, $\beta=0.003, p=0.469$ ), PCC-DLPFC-FC (left: mean \pm SD baseline $=0.24 \pm 0.10$, mean \pm SD follow-up $=0.28 \pm 0.13, \beta=0.035, p=0.162$; right: mean $\pm S D$ baseline $=0.25 \pm 0.11$, mean \pm SD follow-up $=0.26 \pm 0.13, \beta=0.010, p=0.719$ ) over time.

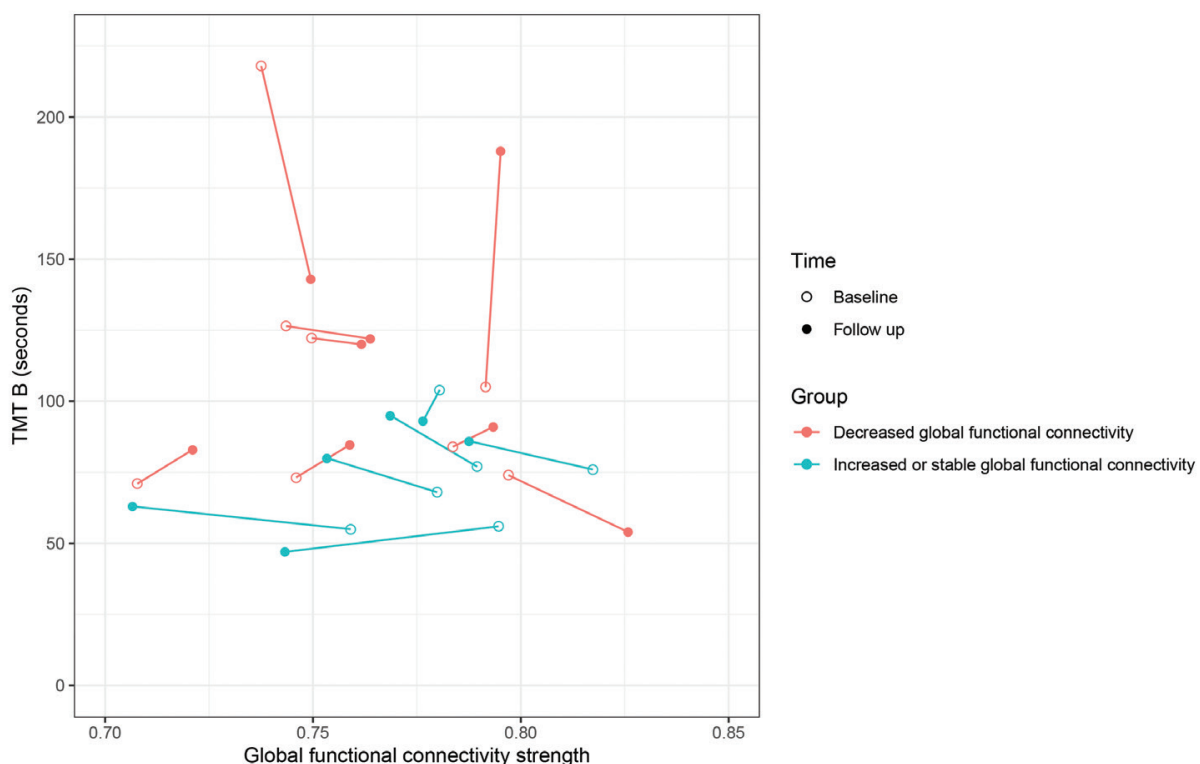

Figure S2. Individual trajectories of delirium patients on global functional connectivity strength and Trail Making Test B (TMT B) score. Preoperative (baseline) and three months postoperative (follow-up) measurements are visualized.

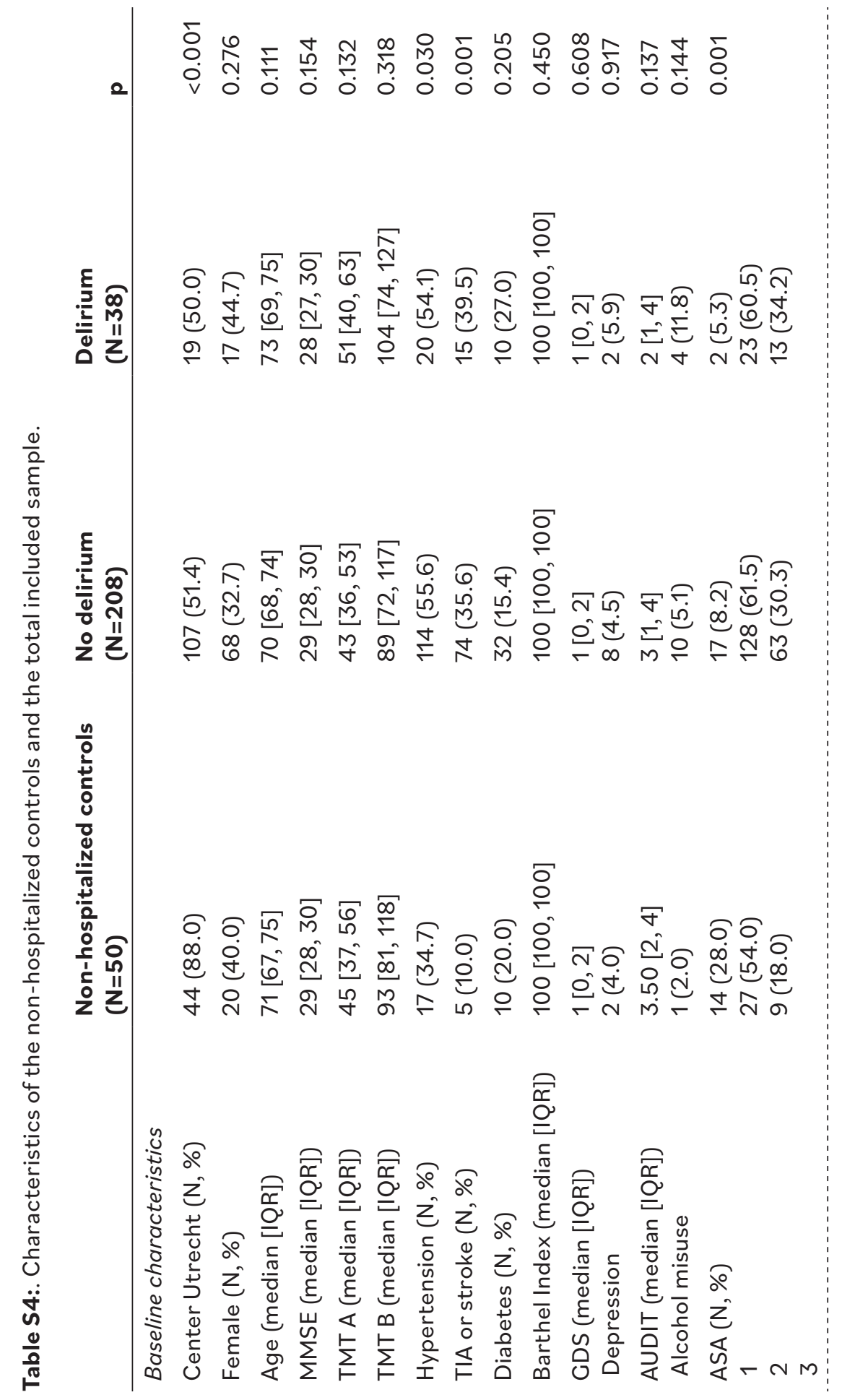




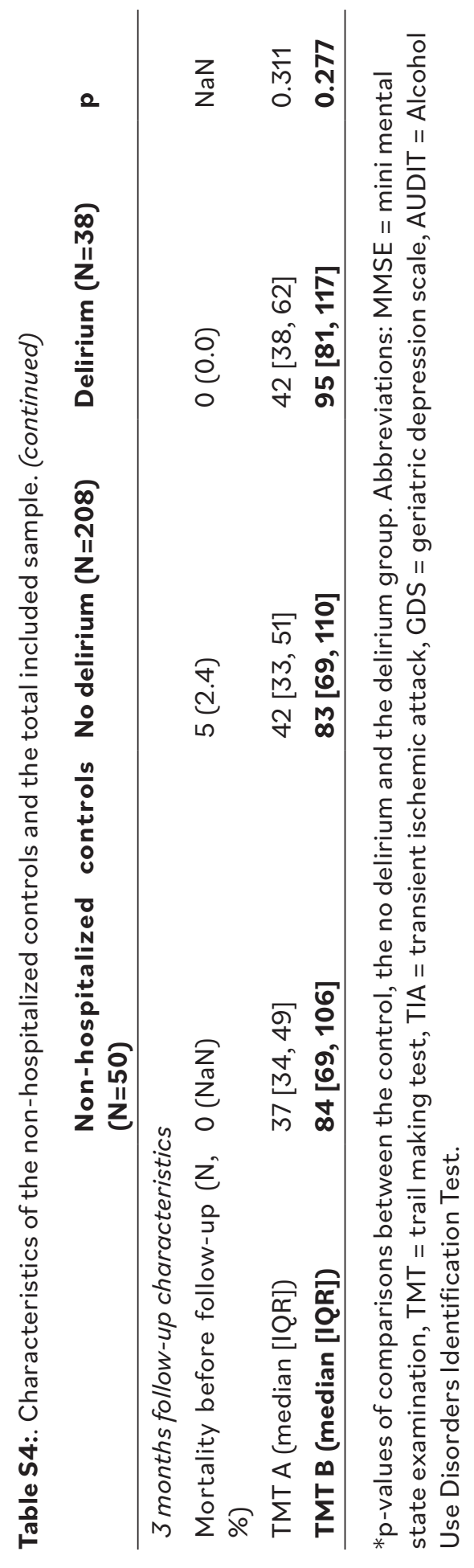

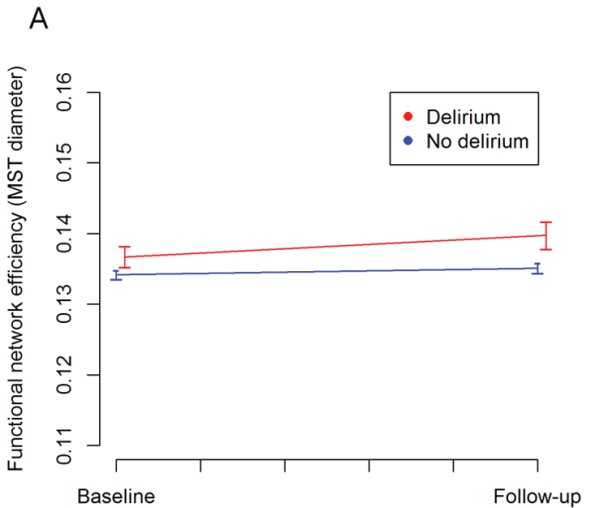

Time

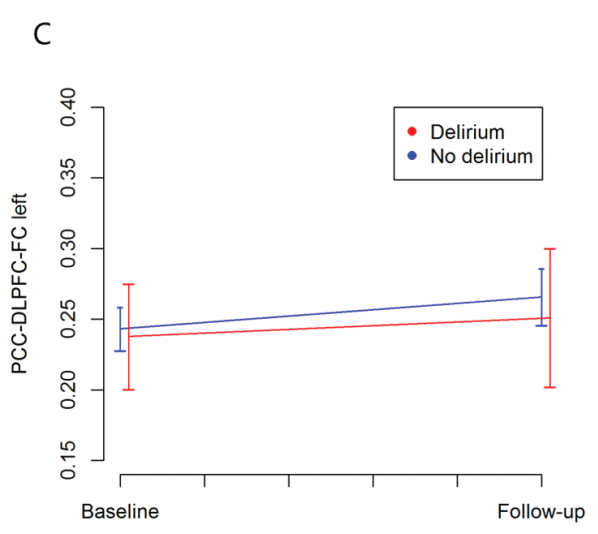

Time

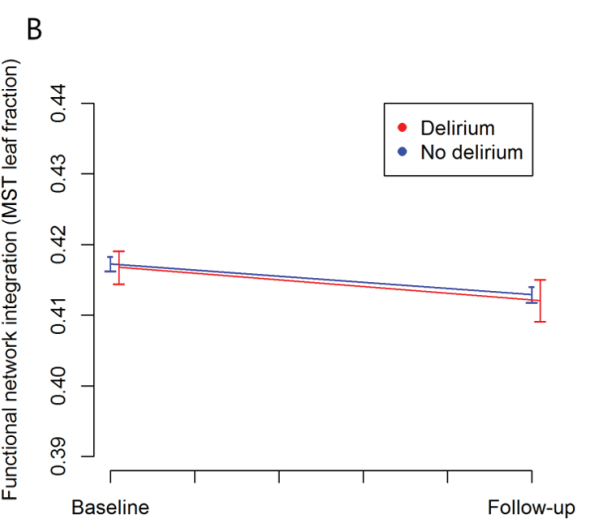

Time

D

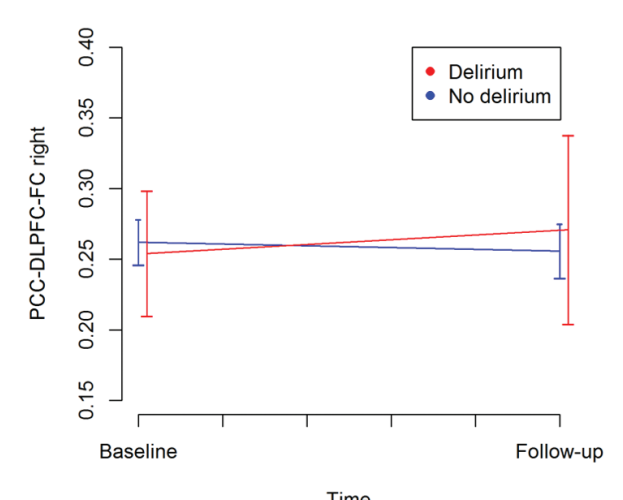

Figure S3. Functional network outcomes over time for the delirium and non-delirium groups. Functional network efficiency (A), functional network integration (B), functional connectivity between the posterior cingulate cortex and the dorsolateral prefrontal cortex left (C) and functional connectivity between the posterior cingulate cortex and the dorsolateral prefrontal cortex right (D) are visualized over time, i.e. at baseline (preoperatively) and at three months follow-up. 


\section{Part 4}

Summary and general discussion 


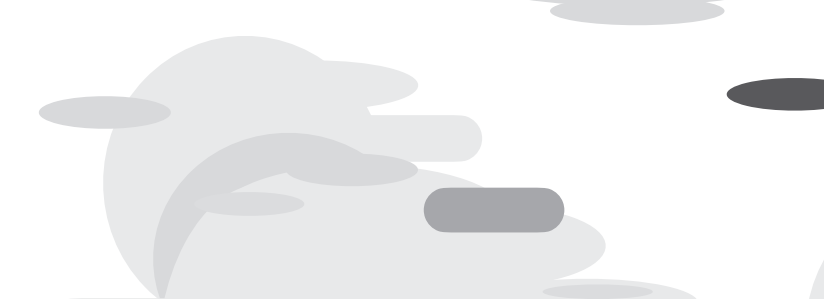

$\odot \odot$

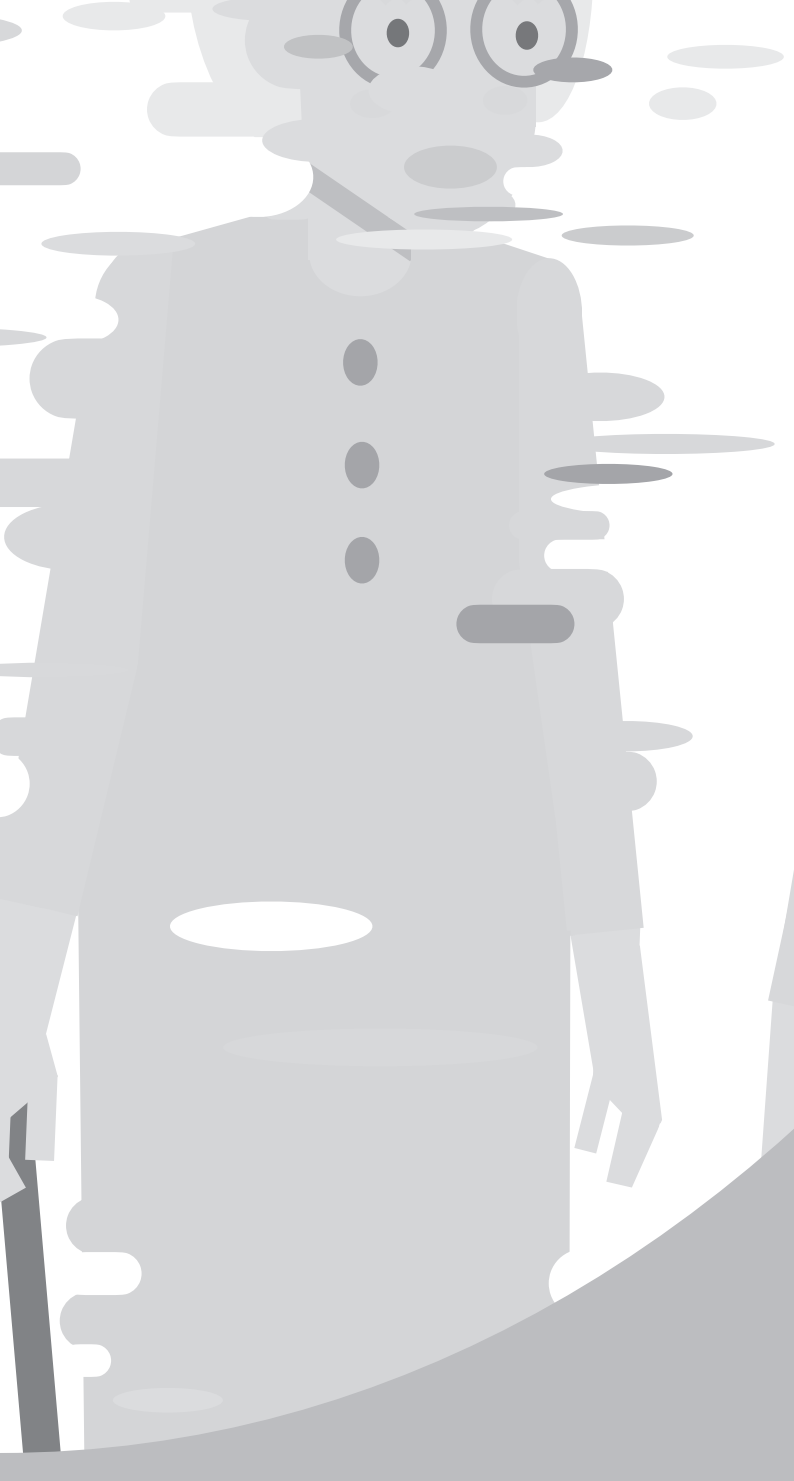

\section{Chapter 8}

\section{Summary}


Delirium is a common neuropsychiatric syndrome, characterized by acute change in attention and awareness, as direct consequence of an underlying medical condition. It is affecting $10-50 \%$ of the hospitalized elderly. Delirium is a burden for patients and related to negative outcomes, such as long-term cognitive impairment. The development of delirium is usually the result of an interaction of various heterogeneous risk factors. Predisposing risk factors, such as older age or cognitive impairment, cover the baseline vulnerability to delirium. Precipitating risk factors for delirium, such as sedation, determine acute changes that can trigger the syndrome. The underlying mechanism of how (combinations of) these risk factors lead to delirium is unknown. In addition, although several hypothesis exist, the pathophysiology of the clinical syndrome is generally unknown. Nevertheless, previous studies have indicated that the acute state of delirium can be accompanied with alterations in brain (network) activity. Studying the brain network in relation to delirium may therefore give us new insights in this complex clinical syndrome. The aim of this dissertation was to evaluate the hypothesis of delirium as a disorder of brain network disintegration. The hypothesis was tested in three different aspects. Brain network disintegration was evaluated as biological substrate of (1) vulnerability for delirium, (2) the clinical syndrome of delirium and (3) longitudinal changes after delirium.

The first part of this dissertation focused on vulnerability for delirium In chapter 2, we tested the hypothesis that delirium and its risk factors are associated with consistent brain network changes, in a systematic review and qualitative meta-analysis of 126 studies. As methodological choices can introduce bias and strongly influence the outcomes of network parameters, we developed a priori quality criteria based on state-of-theart methodological studies and consensus papers from experts in the field. Only studies of good or excellent quality were included in our results. On a structural level, predisposing risk factors were generally associated with lower connectivity strength and less efficient organization of white matter connections. On a functional level, a decrease of functional connectivity strength was found in most studies related to predisposing risk factors. Studies on precipitating factors generally indicated less efficiency of functional networks. During delirium, functional brain networks were characterized by decreased alpha band EEG connectivity strength and lower
fMRI network integration. Taken together, we found evidence that a less connected and less integrated brain network is a common mechanism in the pathophysiology of delirium.

Empirically investigating the integrated effect of delirium risk factors on the functional network may support this hypothesis and may lead to a unified understanding of delirium vulnerability associated with a variety of heterogeneous factors. Therefore, we evaluated the hypothesis that predisposing delirium risk factors induce similar neurophysiological alterations as during delirium in chapter 3 . Elderly subjects $(N=206)$ underwent resting-state EEG measurements and were assessed on predisposing delirium risk factors, i.e. older age, alcohol misuse, cognitive impairment, depression, functional impairment, history of stroke and physical status. Delirium-related EEG characteristics of interest were relative delta power, alpha connectivity strength, and network integration. Functional impairment was found to be associated with decreased alpha connectivity strength. Other predisposing risk factors for delirium had no effect on the studied EEG characteristics. This suggests that predisposition for delirium is not consistently related to EEG characteristics that can be found during delirium. In chapter $\mathbf{4}$, we additionally tested whether predisposing risk factors for delirium are associated with $\mathrm{fMRI}$ network alterations in non-delirious elderly. In this multicenter study, resting-state fMRI data were analyzed from 222 elderly subjects. Functional connectivity strength, network efficiency, and network integration were analyzed, as these measures were altered during delirium in previous studies. We found that predisposing risk factors for delirium were not associated with delirium-related $\mathrm{fMRI}$ network characteristics in an elderly population. Older age within our elderly cohort was related to functional connectivity strength, but in the opposite direction than hypothesized. Delirium-related functional network impairments can therefore not be considered as the common mechanism for predisposition for delirium.

The second part of this dissertation focused on the clinical syndrome of delirium. Previous studies have shown that delirium is associated with decreased functional connectivity and decreased network organization, using EEG. In addition, an altered fMRI connectivity between two specific 
regions that could be involved in cognition, attention or consciousness, i.e. between the posterior cingulate cortex and the dorsolateral prefrontal cortex, has been shown during delirium. In chapter 5, we aimed to increase our understanding of the global organization of the functional network during delirium and to localize possible alterations using fMRI. Restingstate $\mathrm{fMRI}$ data from nine delirious patients, seven post-delirium patients and thirteen non-delirious clinical controls were analyzed. During delirium a decreased functional network efficiency and decreased functional network integration was found. In addition, delirium was associated with loss of hub function, in the right posterior cingulate cortex. Delirium duration was strongly related to loss of functional network integration. After delirium, connectivity strength was decreased and complex regional alterations were found. These findings indicate that delirium reflects disintegration of functional interactions between remote brain areas and suggest longterm impact after the syndrome resolves. As delirium has a fluctuating course, it would be plausible that the disorder does not solely relies upon a static concept. Accordingly, the brain is a dynamic, flexible network that continuously reconfigures depending on the processes and cognitive processes (partly) seem to depend on dynamical functioning or flexibility of the brain. In chapter $\mathbf{6}$, we evaluated the hypothesis that delirium patients have reduced flexibility of the posterior cingulate cortex compared to clinical controls. Flexibility of the right and left posterior cingulate cortex was analyzed in 9 delirious patients and 13 clinical controls. We showed that flexibility of the posterior cingulate cortex did not differ between patients with delirium and clinical controls, indicating that disturbed flexibility might not be a correlate of the acute cognitive problems observed during delirium.

The last part of this dissertation focused on longitudinal changes after delirium. Delirium is associated with an increased risk of long-term cognitive impairment and dementia. Decreased functional connectivity strength and disturbed brain network organization have been described during postoperative delirium, and in patients with dementia. In chapter 7, we studied whether development of postoperative delirium is associated with changes in the functional brain network over time, in an elderly surgery population. Elective surgery patients underwent clinical assessments and resting-state $\mathrm{fMRI}$ before and three months after surgery. Delirium was measured during postoperative hospitalization. fMRI connectivity strength, network efficiency, and network integration were analyzed in 246 patients, of whom 38 (16\%) developed postoperative delirium. We showed that delirium was related to long-term functional connectivity strength decreases in this surgical elderly population, as opposed to increased postoperative connectivity strength in non-delirious controls. In addition, decreased functional connectivity strength was associated with cognitive decline, irrespective of postoperative delirium. We therefore speculate that connectivity strength is related to the increased risk of long-term cognitive impairment and dementia after delirium. 


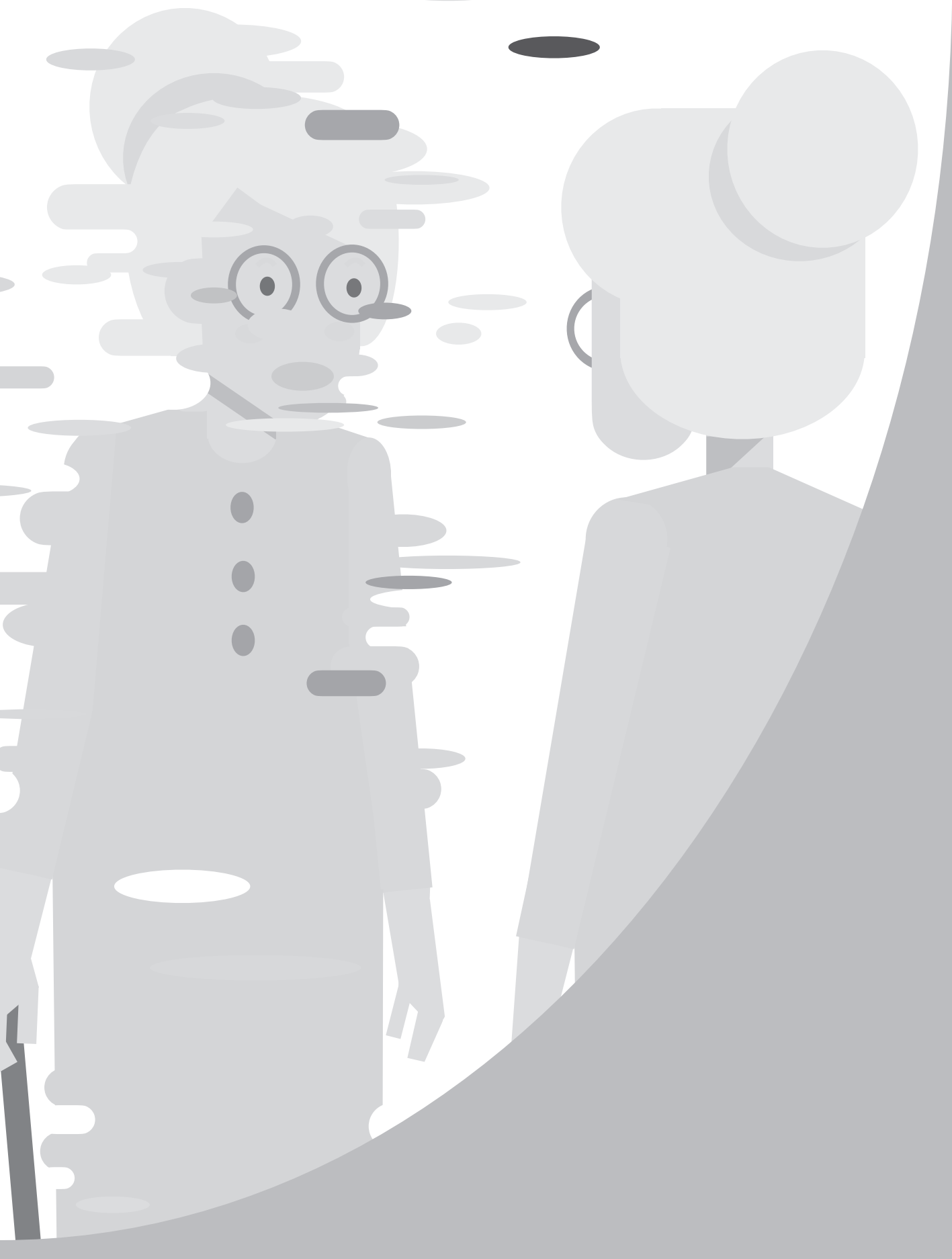

\section{Chapter 9}

General discussion 


\section{Box 1: Clinical case examples}

\section{Patient A}

Patient $A$ is a 78 year old woman, scheduled for elective coronary artery bypass surgery. She lives together with her husband. The last few months, her husband noticed that her memory is not what it used to be, but she does not complain about it. Since several years, she has diabetes type II and hypertension, which are both under control with medication. The surgery is complicated by an arterial bleeding and takes 400 minutes. After surgery, patient $A$ is (according to the protocol) transferred to the Intensive Care. She does not sleep well that night. The next day, she is transferred to the cardiothoracic ward. She is a bit restless and sometimes she forgets how she can contact the nurse. When her husband visits her, she is more at ease. On the third postoperative day, she is confused and does not know where she is. She is drowsy and does not respond adequately during conversations. A geriatrician is consulted and diagnoses a delirium. She does not recover well and two days later an infection is detected. The delirium symptoms last for five days. After ten postoperative days, she is transferred to her local hospital to further recover from surgery. Eight days afterwards, she is back home. Her husband notices that her memory problems are more serious now. After seven months, dementia is diagnosed and soon after the diagnosis she moves into a nursing home.

\section{Patient B}

Patient $B$ is a 75 year old man, scheduled for an elective knee replacement surgery. He lives independently. Since several years, he has been suffering from depressive episodes. He swims twice a week and is, apart from the problems with his knee, in a relatively good physical condition. He does not take any medication. The surgery is uncomplicated and takes 100 minutes. Patient $B$ recovers quickly during the postoperative stay in the hospital. At the fourth postoperative day, he is discharged from the hospital and moves in with his daughter to recover completely. Four weeks later, he comes back home and after two months he starts swimming again.
In this chapter, findings of this dissertation will be discussed in a broader perspective, methodology will be evaluated and suggestions for future directions and clinical implications will be introduced. In Box 1, two clinical case examples are given, which will be used throughout the chapter.

\section{From vulnerability to long-term outcomes}

Risk factors for delirium

Heterogeneous predisposing and precipitating risk factors are expected to cause delirium in a complex interaction ${ }^{1,2}$. Its etiology can therefore be considered as complex and multifactorial. It could be that specific risk factors are more potent in the development of delirium or that specific combinations of risk factors are required. However, at the moment we do not understand the exact interplay between the different factors ${ }^{2-4}$. Accordingly, we do not know if these risk factors share a similar underlying mechanism of how (a combination of) factors lead to delirium and which biological systems are weakened under the influence of the risk factors (chapter 2, 3, 4). Moreover, although different etiologies all lead to the same clinical manifestation of delirium, we currently do not know whether delirium due to different etiologies can be regarded as the same disorder 5. Up to now, no research has been done comparing possible sub-types of delirium that developed after one major precipitating risk factor, e.g. surgery, infection or metabolic disorder.

Understanding the etiology of delirium would be of huge relevance to possibly intervene before delirium can develop. Currently, we know that both patient $A$ and patient $B$ are vulnerable to postoperative delirium. However, we cannot measure on forehand that patient $A$ will develop delirium and patient $B$ will not. In this dissertation, we made a first attempt in unraveling a unified understanding of risk for delirium (chapter 2, 3, 4). Continuing this approach in future research may possibly elucidate the underlying mechanism of this crucial step in the development of delirium.

Studying delirium in clinical practice

Assessing the pathway of development of delirium is difficult considering that it always occurs after a precipitating risk factor and it is mostly unpredictable when this factor will appear. Therefore, only a limited amount 
of observational studies is available studying development of delirium. The Biomarker for Postoperative Cognitive Impairment in the Elderly (BioCog) study (chapter 3, 4, 7), especially aimed for measurements before, during and after delirium, to outline the whole process from vulnerability for delirium to long-term outcomes after delirium. As delirium may develop postoperatively, a surgical study population was used ${ }^{6}$. We assumed that postoperative delirium is comparable to other (possible) sub-types of delirium.

Although this study population is well suited for this type of research, the incidence of postoperative delirium is low (16\% in this dissertation) (chapter 7). To be able to have enough statistical power to detect differences with the non-delirium patients, many patients had to be included in the study. Including a large elderly surgery patient group was a multiannual operation and took a lot of effort and time. Since it is unknown which (baseline) factors are of relevance, it was challenging to gather as much information as possible within the limited time that may be requested from the included patients.

In addition, the BioCog study design aimed to follow up patients three months postoperatively. This is very relevant, as delirium is associated with long-term consequences ${ }^{4}$. However, patients with negative outcomes were generally more often unable to come to the hospital for the followup measurements (chapter 7). Future studies may consider an option to conduct follow-up measures at the patient's home, for example using online or telephonic questionnaires, and if brain activity measures are of interest by using portable EEG devices.

\section{Brain network disintegration as biological characteristic for delirium} In the past decades, several hypotheses have been postulated to explain the complex pathophysiology of delirium. It has been suggested that the syndrome can result from neuroinflammation or neurotransmitter disturbances ${ }^{5}$. A more recent hypothesis proposes delirium as a disconnection syndrome, caused by breakdown of brain networks ${ }^{5,7,8}$. As a starting point for this dissertation, two previous electroencephalography (EEG) studies actually indicated disconnection of the brain network during the syndrome and additionally showed that delirium was associated with altered brain network organization ${ }^{9,10}$. We therefore pursued to gain more insights in the underlying mechanisms of delirium, by studying it as a disorder of brain network integration. Our hypothesis was tested in three different aspects. Brain network disintegration was evaluated as substrate of (1) vulnerability for delirium, (2) the clinical syndrome of delirium and (3) longitudinal changes after delirium.

In our review and meta-analysis, we found some evidence that predisposing risk factors for delirium can be associated with decreased functional connectivity strength (chapter 2). However, in our empirical studies using functional magnetic resonance imaging ( $f M R I)$ and EEG, connectivity alterations reported during delirium do not appear to be a common manifestation in patients with one or more predisposing risk factors (chapter 3,4 ). It is therefore possible that predisposition for delirium is defined by other functional brain (network) characteristics than the profile of delirium itself. On the other hand, it could be that predisposing risk for delirium is solely related to structural network abnormalities, while precipitating risk factors and the fluctuating nature of delirium itself may be characterized by functional network impairments (chapter 2)

It seems that specifically during the clinical syndrome of delirium, functional network efficiency and functional network integration are decreased (chapter 5, 7). Loss of functional network integration was strongly associated with delirium duration. In addition, delirium is related with a loss of hub function of the right posterior cingulate cortex (chapter 5). Dynamical aspects of $\mathrm{fMRI}$ connectivity appear less sensitive to characterize the fluctuating course of delirium than time-invarient functional connectivity measures, such as measures for functional network efficiency or functional network integration (chapter 6). The functional brain network disintegration may therefore be considered as a biological characteristic of delirium (chapter $\mathbf{5 , 7}$ )

After delirium resolution, decreased functional connectivity strength can be observed (chapter 5, 7), while patients without delirium may show increased functional connectivity three months postoperatively (chapter 7). Decreased functional connectivity strength has previously been found in 
patients with cognitive impairment or dementia ${ }^{11-19}$. Accordingly, patients with decreased functional connectivity, irrespective of the occurrence of delirium, are showing declined cognitive performance (chapter 7). These findings indicate long-term impact of delirium on the functional brain network after the syndrome clinically resolves, possibly related to lasting cognitive deterioration

Using $\mathrm{fMRI}$, we were not able to replicate the finding of decreased functional connectivity strength during delirium, as previously found in EEG studies 9,10 , possibly due to the small sample size of our delirium group (chapter 5). We may therefore speculate that topological changes of the functional brain network are more outspoken during delirium, than changes in global functional connectivity strength. Hubs, i.e. central nodes of the network, play an important role in 'higher-order' cognitive tasks and adaptive behavior ${ }^{20,21}$. Therefore, impaired hubs are more likely to be associated with symptoms of brain dysfunction whereas damage to peripheral nodes may be asymptomatic ${ }^{22}$. In addition, hubs are more vulnerable to a diverse range of pathogenic processes ${ }^{23,24}$. Accordingly, disturbances in brain hubs have been previously indicated as a common mechanism in brain disorders 22. It could therefore be that the functional connectivity strength of hubs is impaired first, resulting in topological alterations, together leading to the initiation of delirium symptoms. During this process, the global functional connectivity strength additionally deteriorates. If this deterioration of functional connectivity strength is irreversible, for example due to low cognitive reserves, this may lead to long-term cognitive problems.

Evaluating patient $A$ and patient $B(B \circ \times 1)$ over the perioperative course, thei course of brain networks characteristics would probably have been quite different. In Figure 1, we have hypothetically depicted their brain network characteristics using the information obtained in this dissertation. Please note that the information in the figure and the following description are not observed in reality, as all analyses were done at group level. The content is therefore speculative. In patient A, simultaneously with the development of predisposing risk factors for delirium, i.e. older age and reduced physical status, the structural network starts to deviate (chapter 2). After the occurrence of precipitating risk factors, i.e. coronary artery bypass surgery, functional network efficiency, functional network integration and functional connectivity strength start to alter. At the third postoperative day (indicated in the figure as delirium chance), patient $A$ is delirious and functional network efficiency and functional network integration are seriously impaired (chapter $\mathbf{2}, \mathbf{5}$ ). After recovery of clinical symptoms of delirium, functional connectivity strength alterations are still increasing, eventually leading to the more severe cognitive complaints (chapter $\mathbf{5}, \mathbf{7}$ ). In patient B, structural network characteristics start deviating from the moment that the predisposing risk factors are developing, i.e. older age and depression (chapter 2). As patient B does not develop delirium, no alterations are observed in functional brain network efficiency or functional brain network integration (chapter 2, 5). However, postoperatively, functional connectivity strength start to alter slightly, possibly due to surgery-related neuroinflammation and reduced oxygen supply. After recovery from surgery, still some deviations in functional connectivity strength may be observed (chapter 7)

Taken together, this dissertation may add the following conclusions to the existing literature:

1. Predisposing risk for delirium does not appear to be associated with similar functional network alterations as observed during delirium.

2. Network disintegration can be defined as biological characteristic for the clinical syndrome of delirium. Alterations in functional network efficiency and integration seem to be related to the clinical symptoms of delirium and may recover when delirium resolves.

3. Delirium is associated with a decrease in global connectivity strength of the functional brain network over time. This alteration could be the biological substrate of impaired outcomes of delirium, such as longterm cognitive dysfunction or dementia.

The theory of delirium as a disorder of brain network disintegration does not have to replace other hypotheses on the pathophysiology of delirium. Previous hypotheses indicated neuroinflammation, neurotransmitter 
disturbances, neuronal aging, oxidative stress or neuroendocrine disturbances as an essential underlying biological mechanism for delirium ${ }^{5}$. It remains to be studied to what extent brain network alterations are associated with these other hypotheses. A model study that aimed to explain EEG phenomena seen in delirium, showed that EEG alterations associated with delirium, including connectivity and network impairments, may be the result of imbalance between excitatory and inhibitory activity, as well as increased fluctuations in subcortical information ${ }^{25}$. Particularly an altered balance between glutamatergic and GABAergic neurotransmission may contribute to network vulnerability ${ }^{7}$. Previous studies have shown GABAergic medication, including benzodiazepines, as precipitant of delirium ${ }^{3}$ and reduced network connectivity ${ }^{26}$. Due to the heterogeneity of the etiology, it is unlikely that a single pathway will explain the phenomena of delirium ${ }^{5}$. Presumably, integrating different hypotheses for delirium may be beneficial in elucidating the complex pathophysiology.
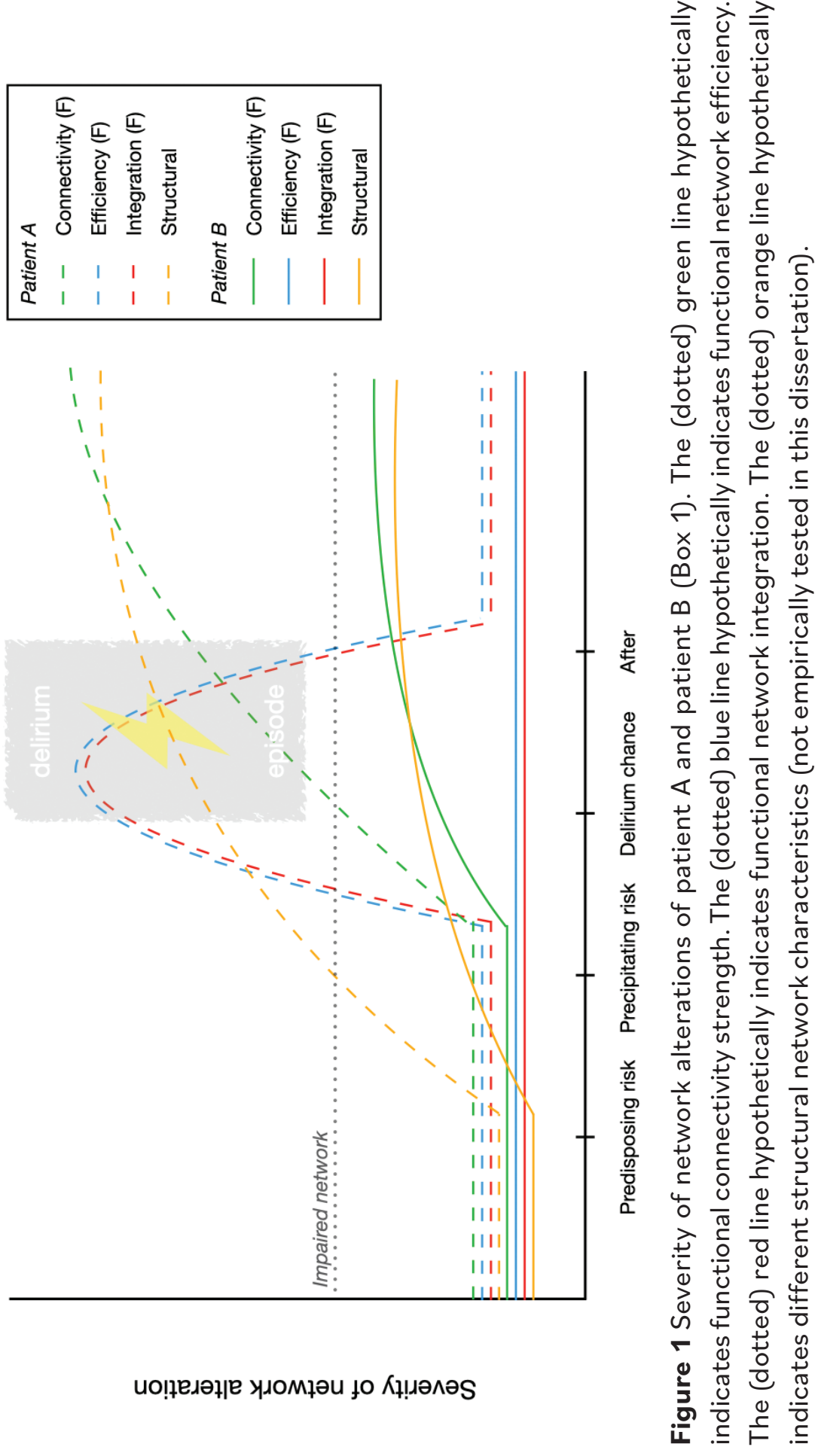


\section{Methodological considerations}

\section{FRI versus EEC}

Using neurophysiological measurements, such as resting state EEG or neuroimaging measurements, such as resting state $\mathrm{FMRI}$ in an elderly (chapter $\mathbf{3}, \mathbf{4 , 7}$ ) or a delirious population (chapter $\mathbf{5 , 6}$ ) is challenging. Both techniques require that participants do not move during the measurements and that participants remain in a 'resting state', while not falling asleep. Delirious patients can be restless or agitated ${ }^{27}$, which may influence the quality of EEG and fMRI measurements (chapter $\mathbf{5 , 6}$ ). In addition, elderly do often experience problems with lying or sitting completely still (chapter $3,4,7$ ).

An advantage of EEG is that the researcher may directly observe the patient during the measurement and at the same time notices the measured signals. If the signal deviates or if the participant moves, the researcher has the possibility to intervene. Although it is very difficult to reconstruct signals contaminated by motion artefacts, the researcher can manually select parts of the signal that are least effected by motion or other noise. For network analyses, 80 seconds of artifact-free data is adequate ${ }^{28}$. As EEG measurements mostly take 5-10 minutes, it is (in most cases) achievable to collect the right amount of artifact-free data.

In contrast, motion seems to be more persistent problem in $\mathrm{fMRI} 29,30$ During $\mathrm{fMRI}$ measurements, no direct information on small movements of the patients is available. It could be an advantage that many motion correction steps are possible after fMRI measurement. However, as we have observed in this dissertation, these motion corrections are often not sufficient, and a considerable part of the participants had to be excluded from the network analyses (chapter 4, 5, 6, 7). On average, fMRI resting state measurements take longer than EEG resting state measurements. An additional structural MRI scan, of 5-10 minutes, is needed to localize the $\mathrm{fMRI}$ signal. The actual fMRI measurement mostly takes between 10-15 minutes. From these fMRI data, at least 4 minutes should be of sufficient quality (after applying motion correction) ${ }^{31}$. The 'motion tolerance' of the signal, is therefore lower in $\mathrm{fMRI}$ than in EEG.
From that point of view, I would consider EEG as a more appropriate technique to measure brain activity than fMRI, to study an elderly or delirious population. In addition, EEG devices are portable, which can be of relevance to measure patients that are unable to visit the hospital for research purposes. Furthermore, EEG has a higher temporal resolution than $\mathrm{fMRI}$. However, $\mathrm{fMRI}$ has a superior spatial resolution compared to EEG, and could therefore be used to integrate functional brain network analysis with neuroanatomical information, such as functional connectivity between specific regions (chapter $\mathbf{4}, \mathbf{5}, \mathbf{6}, \mathbf{7}$ ). This dissertation may show that information obtained using $\mathrm{fMRI}$ can be of huge interest and may add information to EEG related findings. I would therefore state that both techniques are complementary to each other and that use of both is needed to elucidate our understanding on functional brain networks.

Conducting network studies using fMRI

Conducting fMRI network studies (chapter 4, 5, 6, 7), is accompanied with numerous processing steps to consider before the brain network can be calculated. The researcher needs to make somewhat arbitrary choices, for example on several preprocessing, additional motion correction and a parcellation atlas to define the brain regions of the network. Of course, not every research question and dataset is comparable. The variety of options in fMRI (pre-)processing allows a broad range of diverse analyses, which can be a huge benefit. However, fMRI researchers from different groups are currently applying a diversity of steps in conducting similar fMRI network studies, which can significantly influence the results ${ }^{32}$. It is therefore hard to compare studies to each other, and very complicated to set up your own fMRI analysis pipeline. There is an urgent need for consensus on fMRI (pre-) processing steps to improve the comparability between different fMR network studies.

Challenges in network science

Network science allows us to study network characteristics of the healthy and diseased brain. However, some difficulties emerge by studying the brain network due to various methodological choices, for example the use of adequate connectivity measures ${ }^{28,33-35}$ and the definition of nodes and edges $^{36-40}$. These methodological choices can introduce bias and strongly 
influence the outcomes of network analyses ${ }^{28,34,41,42}$. In chapter $\mathbf{2}$, we have evaluated the most common problems and defined quality criteria based on state-of-the-art methodological studies and consensus papers from experts $3,28,29,31,34$. These quality criteria can be used and adapted for future investigations.

In the empirical studies of the dissertation (chapter 3, 4, 5, 6, 7), we have used the minimum spanning tree (MST). The MST can be considered as the backbone network and connects all nodes with the highest possible weights without forming loops ${ }^{43,44}$. Therefore, the MST always consists of a fixed number of connections, which avoids the methodological bias of spurious connections or arbitrary thresholding in group comparisons of network topology. The MST has a high overlap with more common global network characteristics based, such as path length and clustering coefficient ${ }^{43}$. Even for evaluation of modules in the network, the MST seems appropriate ${ }^{45}$ Use of the MST in future network studies can therefore be recommended to (partly) avoid methodological biases.

Selection bias \& sample size

As the BioCog study protocol, of which data was used in chapter 3, 4 and 7, was very extensive, i.e. two times a four hour hospital visit, including $\mathrm{MRI}$ and EEG measurements (pre- and three months postoperatively), three extra blood drawings and twice daily visits from the study team during hospitalization, the included population may not be generalizable to a standard elderly population scheduled for elective surgery ${ }^{6}$. Patients with (a few) cognitive impairments, patients with a higher disease burden, and patients that did not have abilities to travel individually, i.e. in general patients that could be more vulnerable to develop delirium, are likely underrepresented in our study population. This may have led to an underestimation of our results. Furthermore, the sample sizes of the delirium group used in this dissertation were small (chapter 5, 6,7), which may have reduced the statistical power of our analyses.
Network disintegration in clinical practice \& future perspectives

\section{EEG-based delirium detection}

Delirium is often not properly detected, especially its hypoactive subtype ${ }^{4,46}$. The longer a patient is delirious, the more difficult it becomes to treat the syndrome and the higher the probability that a patient develops worse outcomes, such as long-term cognitive impairment ${ }^{47}$. If delirium is detected in time, medical care, pharmacological and non-pharmacological, can be effective in treatment of the disorder ${ }^{48,49}$. Due to the fluctuating nature and the sometimes subtle symptoms, it takes effort to correctly detect delirium. In addition, the screening methods that are currently used seem to work sufficiently in a research setting, but disappoint in routine clinical practice ${ }^{50}$. Furthermore, the diagnosis of delirium can additionally be difficult for experts, as there appeared to be considerable disagreement in its classification by experts who independently evaluated exactly the same information ${ }^{51}$.

In the past few years, our research group has successfully evaluated the opportunities for one-channel EEG-based delirium detection ${ }^{52,53}$. The onechannel EEG patch can be easily placed at the patient's head and within minutes the delirium likelihood is objectively computed based on EEC characteristics. It may even detect delirium before the clinical symptoms can be observed ${ }^{53}$. Patient A (Box 1) already showed some delirium-related symptoms at the second postoperative day. If delirium was detected earlier and therefore treatment was started earlier, this may have resulted in a different course of (consequences of) delirium.

Currently, a large clinical evaluation study is conducted on one-channe EEG-based delirium detection. If EEG-based delirium detection appears to function properly in clinical practice, this could lead to a huge improvement of delirium detection, which consequently may have beneficial impact on delirium patients, their families and caregivers. This may emphasize the importance of studying biological mechanisms of the brain during delirium and its potential use in clinical practice. 
EEC-based personalized delirium treatment

Delirium is characterized by functional network disintegration (chapter 5) and slowing of the dominant EEG oscillations ${ }^{10}$. In contrast with the level of a blood biomarker, EEG is a multidimentional signal. EEG measurements during delirium may therefore be used to define personalized delirium treatment. As mentioned above, $\mathrm{fMRI}$ may be more sensitive to motion and is not portable. Therefore, especially use of EEG can be further studied in this regard. Importantly, our research group has shown that it is feasible to perform EEG recordings in delirium patients ${ }^{9,10}$. EEG has been shown as a useful marker for personalized treatment in psychiatric disorders, such as depression and Attention Deficit Hyperactivity Disorder ${ }^{54}$. Future studies should evaluate the potential of EEG measurements during delirium as prognostic and personalized marker of responsiveness to treatment, delirium duration, and long-term cognitive outcome in delirium.

Neurostimulation as treatment for delirium

Currently, delirium treatment is mainly focused on curing the underlying medical conditions, but in 30-50\% of patients, delirium persists when these factors are resolved ${ }^{55}$. Since delirium is characterized by a disintegrated functional network and long-term decreased functional connectivity (chapter 5,7 ), studies on delirium treatment may focus on normalization of these brain alterations. Transcranial direct current stimulation (tDCS) is a noninvasive neurostimulation technique that is being increasingly explored for the treatment of neurological and psychiatric symptoms ${ }^{56}$. Different observations suggest that tDCS has great potential as delirium treatment. In a rat model for postoperative delirium, tDCS treatment positively impacted acute attention deficits, the core symptom of delirium, and reversed delirium-associated EEG slowing ${ }^{57}$. Furthermore, in healthy humans, tDCS increased alpha band activity, functional connectivity, and network efficiency, which would possibly mean a reversal of pathological alterations in delirium ${ }^{58-62}$. In addition, tDCS is effective as treatment of conditions that are related to delirium such as depression, cognitive impairment and auditory verbal hallucinations ${ }^{63-66}$. tDCS is a safe treatment, side effects are very mild and rarely reported ${ }^{67}$. Other benefits include that tDCS equipment is relatively cheap and that the device is portable. It would therefore be of huge interest to study the effectiveness of tDCS for delirium treatment.

\section{Conclusion}

Taken together, this dissertation concludes that predisposing risk for delirium does not appear to be associated with similar functional network alterations as observed during delirium. In addition, network disintegration can be defined as biological characteristic for the clinical syndrome of delirium. Alterations in functional network efficiency and integration seem to be related to the clinical symptoms of delirium and may recover when delirium resolves. Furthermore, delirium is associated with a decrease in global connectivity strength of the functional brain network over time. This alteration could be the biological substrate of impaired outcomes of delirium, such as long-term cognitive dysfunction or dementia. 


\section{References}

1. MaldonadoJR. Delirium pathophysiology: An updated hypothesis of the etiology of acute brain failure. Int J Geriatr Psychiatry. 2018;33(11):1428-1457. doi:10.1002/ gps.4823

2. Inouye SK, Westendorp RG, Saczynski JS. Delirium in elderly people. Lancet 2014;383(9920):911-922. doi:10.1016/S0140-6736(13)60688-1

3. Zaal IJ, Devlin JW, Peelen LM, Slooter AJC. A Systematic Review of Risk Factors for Delirium in the ICU*. Crit Care Med. 2015;43(1):40-47. doi:10.1097/ CCM.0000000000000625

4. Marcantonio ER. Delirium in Hospitalized Older Adults. Solomon CG, ed. N EnglJ Med. 2017;377(15):1456-1466. doi:10.1056/NEJMcp1605501

5. MaldonadoJR. Neuropathogenesis of delirium: review of current etiologic theories and common pathways. Am J Geriatr Psychiatry. 2013;21(12):1190-1222. doi:10.1016/j. jagp.2013.09.005

6. Winterer G, Androsova G, Bender O, et al. Personalized risk prediction of postoperative cognitive impairment - rationale for the EU-funded BioCog project. Eur Psychiatry. 2018. doi:10.1016/j.eurpsy.2017.10.004

7. Sanders RD. Hypothesis for the pathophysiology of delirium: Role of baseline brain network connectivity and changes in inhibitory tone. Med Hypotheses. 2011;77(1):140 143. doi:10.1016/j.mehy.2011.03.048

8. Young JWS. The network model of delirium. Med Hypotheses. 2017;104:80-85 doi:10.1016/j.mehy.2017.05.027

9. Numan T, Slooter AJC, van der Kooi AW, et al. Functional connectivity and network analysis during hypoactive delirium and recovery from anesthesia. Clin Neurophysiol. 2017;128(6):914-924. doi:10.1016/j.clinph.2017.02.022

10. van Dellen E, van der Kooi AW, Numan T, et al. Decreased Functional Connectivity and Disturbed Directionality of Information Flow in the Electroencephalography of Intensive Care Unit Patients with Delirium after Cardiac Surgery. Anesthesiology. 2014;121(2):328-335. doi:10.1097/ALN.0000000000000329

11. Carlos G, Stam CJ, Gómez C, et al. Disturbed Beta Band Functional Connectivity in PatientsWith Mild Cognitive Impairment: An MEG Study. IEEE Trans Biomed Eng. 2009;56(6):1683-1690. doi:10.1109/TBME.2009.2018454

12. van Dellen E, de Waal H, van der Flier WM, et al. Loss of EEGNetwork Efficiency Is Related to Cognitive Impairment in Dementia With Lewy Bodies. Mov Disord. 2015;30(13):1785-1793. doi:10.1002/mds.26309
13. Filippi M, Basaia S, Canu E, et al. Brain network connectivity differs in earlyonset neurodegenerative dementia. Neurology. 2017;89(17):1764-1772. doi:10.1212/ WNL.0000000000004577

14. Peraza LR, Taylor J-P, Kaiser M. Divergent brain functional network alterations in dementia with Lewy bodies and Alzheimer's disease. Neurobio/ Aging. 2015;36(9):2458 2467. doi:10.1016/j.neurobiolaging.2015.05.015

15. Minati L, Chan D, Mastropasqua C, et al. Widespread alterations in functional brain network architecture in amnestic mild cognitive impairment.J Alzheimers Dis. 2014;40(1):213-220. doi:10.3233/jad-131766

16. Chang T-Y, Huang K-L, Ho M-Y, et al. Graph theoretical analysis of functional networks and its relationship to cognitive decline in patients with carotid stenosis. Cereb Blood Flow Metab. 2016;36(4):808-818. doi:10.1177/0271678X15608390

17. Zeng K, Wang Y, Ouyang G, Bian Z, Wang L, Li X. Complex network analysis of resting state EEG in amnestic mild cognitive impairment patients with type 2 diabetes. Front Comput Neurosci. 2015;9:133. doi:10.3389/fncom.2015.00133

18. Frantzidis CA, Vivas AB, Tsolaki A, Klados MA, Tsolaki M, Bamidis PD. Functiona disorganization of small-world brain networks in mild Alzheimer's disease and amnestic Mild cognitive impairment: An EEG study using Relative Wavelet Entropy (RWE). Front Aging Neurosci. 2014;6:224. doi:10.3389/fnagi.2014.00224

19. Koenig T, Prichep L, Dierks T, et al. Decreased EEG synchronization in Alzheimer's disease and mild cognitive impairment. Neurobiol Aging. 2005;26(2):165-171. doi:10.1016/j.neurobiolaging.2004.03.008

20. Heuvel M Van Den, Stam C, ... RK-J of, 2009 undefined. Efficiency of functiona brain networks and intellectual performance. Soc Neurosci. https://www. jneurosci.org/content/29/23/7619?utm_source=Lifehacker+Newsletter\&utm campaign $=562729$ ec45-UA-142218-18utm_medium=email. Accessed April 23, 2020.

21. Crossley N, Mechelli A, ... PV-P of the, 2013 undefined. Cognitive relevance of the community structure of the human brain functional coactivation network. Nat/ Acad Sci. https://www.pnas.org/content/110/28/11583.short. Accessed April 23, 2020

22. Crossley NA, Mechelli A, Scott J, et al. The hubs of the human connectome are generally implicated in the anatomy of brain disorders. Brain. 2014;137(8):2382-2395. doi:10.1093/brain/awu132

23. van den Heuvel MP, Sporns O. Rich-Club Organization of the Human Connectome. J Neurosci. 2011;31(44). http://www.jneurosci.org.proxy.library.uu.nl/ content/31/44/15775.short. Accessed July 24, 2017. 
24. Liang X, Zou Q, He Y, Yang Y. Coupling of functional connectivity and regional cerebral blood flow reveals a physiological basis for network hubs of the human brain Proc Natl Acad Sci U S A. 2013;110(5):1929-1934. doi:10.1073/pnas.1214900110

25. Ponten SC, Tewarie P, Slooter AJC, Stam CJ, van Dellen E. Neural Network Modeling of EEG Patterns in Encephalopathy. J Clin Neurophysiol. 2013;30(5):545 552. doi:10.1097/WNP.0b013e3182a73e16

26. Ferrarelli F, Massimini M, Sarasso S, et al. Breakdown in cortical effective connectivity during midazolam-induced loss of consciousness. Proc Nat/ Acad Sci U S A. 2010;107(6):2681-2686. doi:10.1073/pnas.0913008107

27. American Psychiatric Association. Diagnostic and Statistical Manual of Mental Disorders. American Psychiatric Association; 2013. doi:10.1176/appi. books.9780890425596

28. van Diessen E, Numan T, van Dellen E, et al. Opportunities and methodologica challenges in EEG and MEG resting state functional brain network research. Clin Neurophysiol. 2015;126(8):1468-1481. doi:10.1016/j.clinph.2014.11.018

29. Ciric R, Wolf DH, Power JD, et al. Benchmarking of participant-level confound regression strategies for the control of motion artifact in studies of functiona connectivity. Neuroimage. 2017;154:174-187. doi:10.1016/j.neuroimage.2017.03.020

30. Power JD, Barnes KA, Snyder AZ, Schlaggar BL, Petersen SE. Spurious but systematic correlations in functional connectivity MRI networks arise from subject motion. Neuroimage. 2012;59(3):2142-2154. doi:10.1016/j.neuroimage.2011.10.018

31. Birn, R.M., Molloy, E.K., Patriat, R., Parker, T., Meier, T.B., Kirk, G.R., Nair, V.A., Meyerand, M.E., Prabhakaran V. The effect of scan length on the reliability of resting state fMRI connectivity estimates. Neuroimage. 2013;83:550-558. doi:10.1016/J. NEUROIMAGE.2013.05.099

32. Andellini M, Cannatà V, Gazzellini S, ... BB-J of neuroscience, 2015 undefined. Test-retest reliability of graph metrics of resting state MRI functional brain networks: A review. Elsevier. https://www.sciencedirect.com/science/article/pii/ S0165027015002113. Accessed April 19, 2020

33. Chunmei Zhang, Wenxue Li, Ke Wang. Graph Theory-Based Approach for Stability Analysis of Stochastic Coupled Systems With Levy Noise on Networks. IEEE Trans Neural Networks Learn Syst. 2015;26(8):1698-1709. doi:10.1109/TNNLS.2014.2352217

34. Fornito A, Zalesky A, Breakspear M. Graph analysis of the human connectome: Promise, progress, and pitfalls. Neuroimage. 2013;80:426-444. doi:10.1016/j neuroimage.2013.04.087
35. Rubinov M, Sporns O. Complex network measures of brain connectivity: Uses and interpretations. Neuroimage. 2010;52(3):1059-1069. doi:10.1016/j. neuroimage.2009.10.003

36. Power JD, Schlaggar BL, Petersen SE. Recent progress and outstanding issued in motion correction resting state fmri. Neuroimage. 2015;105:536-551. doi:10.1016/j. neuroimage.2014.10.044. Recent

37. Pruim RHR, Mennes M, Buitelaar JK, Beckmann CF. Evaluation of ICA-AROMA and alternative strategies for motion artifact removal in resting state fMRI. Neuroimage. 2015;112:278-287. doi:10.1016/j.neuroimage.2015.02.063

38. Dijk KRA Van, Sabuncu MR, Buckner RL. Neurolmage The in $\mathrm{fl}$ uence of head motion on intrinsic functional connectivity MRI. Neuroimage. 2012;59(1):431-438. doi:10.1016/j.neuroimage.2011.07.044

39. van Wijk BCM, Stam CJ, Daffertshofer A, Stam C, Apkarian A. Comparing Brain Networks of Different Size and Connectivity Density Using Graph Theory. Sporns O, ed. PLoS One. 2010;5(10):e13701. doi:10.1371/journal.pone.0013701

40. van den Heuvel MP, de Lange SC, Zalesky A, Seguin C, Yeo BTT, Schmidt R Proportional thresholding in resting-state fMRI functional connectivity networks and consequences for patient-control connectome studies: Issues and recommendations. Neuroimage. 2017;152:437-449. doi:10.1016/J.NEUROIMAGE.2017.02.005

41. Alderson-Day B, Diederen K, Fernyhough C, et al. Auditory Hallucinations and the Brain's Resting-State Networks: Findings and Methodological Observations. Schizophr Bull. 2016;42(5):1110-1123. doi:10.1093/schbul/sbw078

42. Tijms BM, Wink AM, de Haan W, et al. Alzheimer's disease: connecting findings from graph theoretical studies of brain networks. Neurobio/ Aging. 2013;34(8):2023 2036. doi:10.1016/j.neurobiolaging.2013.02.020

43. Tewarie P, van Dellen E, Hillebrand A, Stam CJ. The minimum spanning tree: An unbiased method for brain network analysis. Neuroimage. 2015;104:177-188. doi:10.1016/j.neuroimage.2014.10.015

44. Stam CJ, Tewarie P, Van Dellen E, van Straaten ECW, Hillebrand A, Van Mieghem $P$. The trees and the forest: Characterization of complex brain networks with minimum spanning trees. Int J Psychophysiol. 2014;92(3):129-138. doi:10.1016/j. jpsycho.2014.04.001

45. Yu M, Hillebrand A, Tewarie P, et al. Hierarchical clustering in minimum spanning trees. Chaos An Interdiscip J Nonlinear Sci. 2015;25(2):023107. doi:10.1063/1.4908014

46. Slooter A, Leur R Van De, neurology IZ-H of clinical, 2017 undefined. Delirium in critically ill patients. Elsevier. https://www.sciencedirect.com/science/article/pii/ B9780444635990000259. Accessed April 19, 2020. 
47. Fong TG, Tulebaev SR, Inouye SK. Delirium in elderly adults: diagnosis, prevention and treatment. Nat Rev Neurol. 2009;5(4):210-220. doi:10.1038/nrneurol.2009.24

48. Barr J, Fraser G, Puntillo K, ... EE-C care, 2013 undefined. Clinical practice guidelines for the management of pain, agitation, and delirium in adult patients in the intensive care unit. journals./ww.com. https://journals.Iww.com/ccmjournal/ Fulltext/2013/01000/Clinical_Practice_Guidelines_for_the_Management_of.29.aspx. Accessed April 19, 2020.

49. Young J, Murthy L, Westby M, Akunne A, Bmj RO-, 2010 undefined. Diagnosis, prevention, and management of delirium: summary of NICE guidance. bmj.com. https:// www.bmj.com/content/341/bmj.c3704.short. Accessed April 19, 2020.

50. van Eijk MM, van den Boogaard M, van Marum RJ, et al. Routine Use of the Confusion Assessment Method for the Intensive Care Unit. AmJ Respir Crit Care Med. 2011;184(3):340-344. doi:10.1164/rccm.201101-00650C

51. Numan T, van den Boogaard M, Kamper AM, Rood PJT, Peelen LM, Slooter AJC Recognition of Delirium in Postoperative Elderly Patients: A Multicenter Study. J Am Geriatr Soc. 2017;65(9):1932-1938. doi:10.1111/jgs.14933

52. van der Kooi AW, Zaal IJ, Klijn FA, et al. Delirium Detection Using EEG. Chest. 2015;147(1):94-101. doi:10.1378/CHEST.13-3050

53. Numan T, Boogaard M van den, ... AK-B journal of, 2019 undefined. Delirium detection using relative delta power based on 1-minute single-channel EEG: a multicentre study. Elsevier. https://www.sciencedirect.com/science/article/pii/ S0007091218306937. Accessed April 19, 2020.

54. Olbrich S, Van Dinteren R, Arns M. Personalized Medicine: Review and Perspectives of Promising Baseline EEG Biomarkers in Major Depressive Disorder and Attention Deficit Hyperactivity Disorder. Neuropsychobiology. 2016;72(3-4):229-240. doi:10.1159/000437435

55. Cole MG, Ciampi A, Belzile E, Zhong L. Persistent delirium in older hospital patients: a systematic review of frequency and prognosis. Age Ageing. 2008;38(1):19 26. doi:10.1093/ageing/afn253

56. Boggio P, Rigonatti S, ... RR-IJ, 2008 undefined. A randomized, double-blind clinical trial on the efficacy of cortical direct current stimulation for the treatment of major depression. academic.oup.com. https://academic.oup.com/ijnp/articleabstract/11/2/249/767876. Accessed April 18, 2020.

57. OhJ, Ham J, Cho D, ParkJY, Kim J-J, Lee B. The Effects of Transcranial Direct Current Stimulation on the Cognitive and Behavioral Changes After Electrode Implantation Surgery in Rats. Front Psychiatry. 2019;10:291. doi:10.3389/fpsyt.2019.00291
58.

Vecchio F, Pellicciari MC, Miraglia F, Brignani D, Miniussi C, Rossini PM. Effects of transcranial direct current stimulation on the functional coupling of the sensorimotor cortical network. Neuroimage. 2016. doi:10.1016/j.neuroimage.2016.01.051

59. Vecchio F, Di lorio R, Miraglia F, et al. Transcranial direct current stimulation generates a transient increase of small-world in brain connectivity: an EEG graph theoretical analysis. Exp Brain Res. 2018. doi:10.1007/s00221-018-5200-z

60. Mancini M, Brignani D, Conforto S, Mauri P, Miniussi C, Pellicciari MC. Assessing cortical synchronization during transcranial direct current stimulation: A graphtheoretical analysis. Neuroimage. 2016. doi:10.1016/j.neuroimage.2016.06.003

61. Wilson TW, McDermott TJ, Mills MS, Coolidge NM, Heinrichs-Graham E. tDCS Modulates Visual Gamma Oscillations and Basal Alpha Activity in Occipital Cortices: Evidence from MEG. Cereb Cortex. 2017. doi:10.1093/cercor/bhx055

62. Mangia AL, Pirini M, Cappello A. Transcranial direct current stimulation and power spectral parameters: a tDCS/EEG co-registration study. Front Hum Neurosci. 2014. doi:10.3389/fnhum.2014.00601

63. Brunoni AR, Moffa AH, Fregni F, et al. Transcranial direct current stimulation for acute major depressive episodes: Meta-analysis of individual patient data. $\mathrm{BrJ}$ Psychiatry. 2016. doi:10.1192/bjp.bp.115.164715

64. Dedoncker J, Brunoni AR, Baeken C, Vanderhasselt M-A. A Systematic Review and Meta-Analysis of the Effects of Transcranial Direct Current Stimulation (tDCS) Over the Dorsolateral Prefrontal Cortex in Healthy and Neuropsychiatric Samples: Influence of Stimulation Parameters. Brain Stimul. 2016. doi:10.1016/j.brs.2016.04.006

65. Brunelin J, Mondino M, Gassab L, et al. Examining transcranial direct-current stimulation (tDCS) as a treatment for hallucinations in schizophrenia. AmJ Psychiatry. 2012;169(7):719-724. doi:10.1176/appi.ajp.2012.11071091

66. Flöel A. TDCS-enhanced motor and cognitive function in neurological diseases. Neuroimage. 2014. doi:10.1016/j.neuroimage.2013.05.098

67. Bikson M, Grossman P, Thomas C, et al. Safety of Transcranial Direct Curren Stimulation: Evidence Based Update 2016. Brain Stimul. 2016. doi:10.1016/j. brs.2016.06.004 


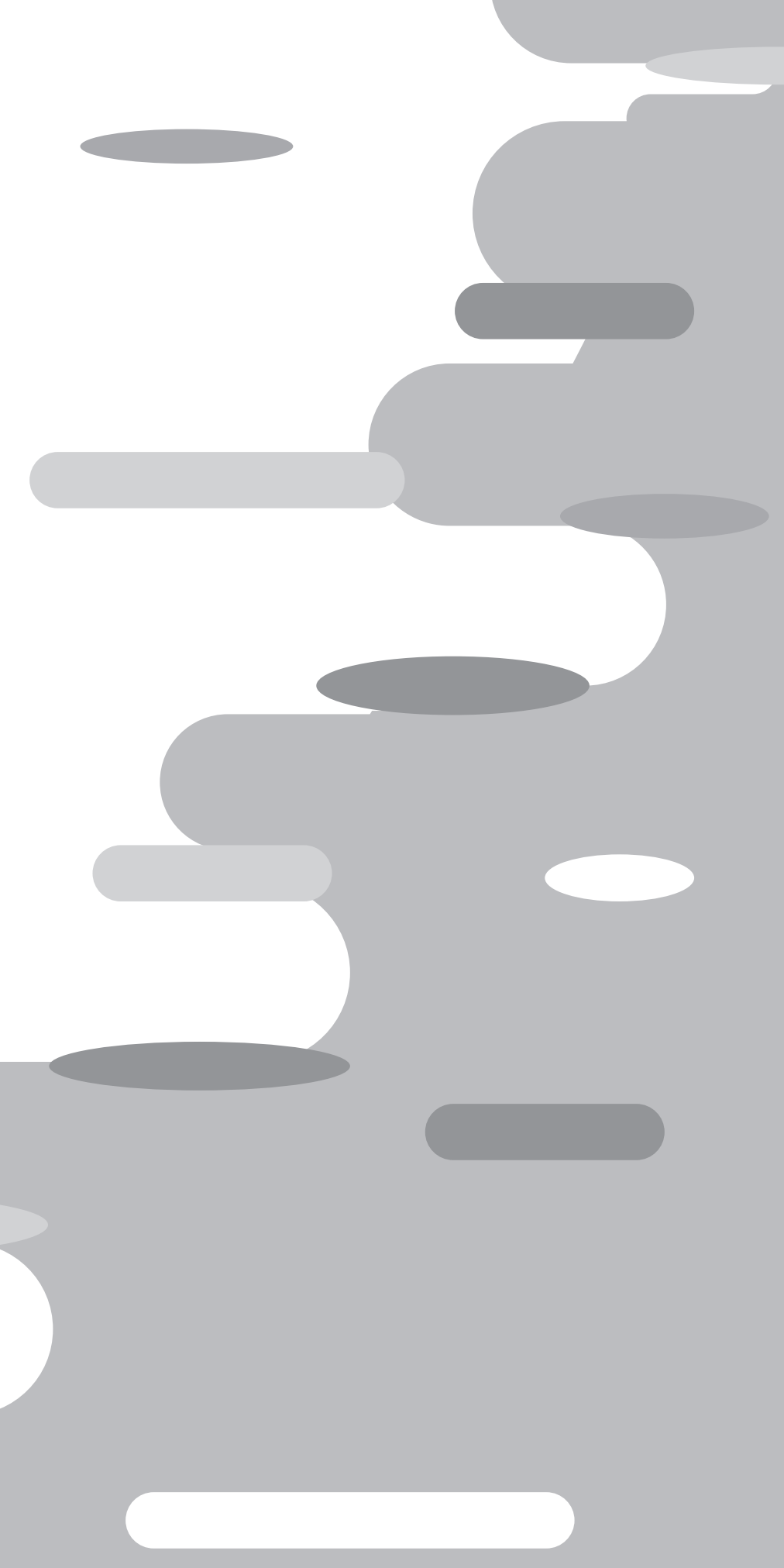

A

Appendices 


\section{Delirium als een stoornis van desintegratie van het hersennetwerk}

Delirium is een veelvoorkomend neuropsychiatrisch syndroom, gekenmerkt door acute veranderingen in aandacht en bewustzijn, als direct gevolg van een onderliggende medische aandoening. Het komt voor bij 10-50\% van de ouderen die in het ziekenhuis opgenomen zijn. Delirium is erg belastend voor patiënten en gerelateerd aan negatieve uitkomsten, zoals langdurige cognitieve problemen. De ontwikkeling van delirium is meestal het resultaat van een interactie van verschillende heterogene risicofactoren. Predisponerende risicofactoren, zoals oudere leeftijd of cognitieve problemen, zorgen voor een basiskwetsbaarheid voor delirium. Precipiterende risicofactoren, zoals sedatie, zorgen voor acute veranderingen die delirium kunnen veroorzaken. Het onderliggende mechanisme van hoe (combinaties van) deze risicofactoren tot delirium kunnen leiden, is tot nu toe onbekend. Hoewel er verschillende hypothesen bestaan, is de pathofysiologie van het klinische syndroom voor het grootste gedeelte onduidelijk. Eerdere studies hebben aangetoond dat de acute toestand van het delirium gepaard kan gaan met veranderingen van (netwerk)activiteit in de hersenen. Het bestuderen van het hersennetwerk in relatie tot delirium zou ons daarom nieuwe inzichten kunnen geven in deze complexe aandoening. Het doel van dit proefschrift was om de hypothese van delirium als een stoornis van desintegratie van het hersennetwerk te evalueren. Deze hypothese is getest op drie verschillende onderdelen, namelijk desintegratie van het hersennetwerk als biologisch substraat van (1) kwetsbaarheid voor delirium, (2) het klinische syndroom van delirium zelf en (3) longitudinale veranderingen na delirium.

In het eerste gedeelte van dit proefschrift werd kwetsbaarheid voor delirium behandeld. In hoofdstuk 2 hebben we geëvalueerd of delirium en risicofactoren voor delirium gerelateerd zijn aan consistente veranderingen in het hersennetwerk, met behulp van een systematische review en kwalitatieve meta-analyse van 126 studies. Aangezien methodologische keuzes bias kunnen introduceren en de uitkomsten van netwerkparameters sterk kunnen beïnvloeden, hebben we van tevoren kwaliteitscriteria ontwikkeld, op basis van recent ontwikkelde methodologische studies en consensus artikelen van experts in het veld. Alleen studies van goede of excellente methodologische kwaliteit werden in onze resultaten opgenomen. Op structureel niveau waren predisponerende risicofactoren doorgaans gerelateerd aan een verminderde netwerksterkte en een minder efficiënte organisatie van verbindingen in de witte stof. Op functioneel niveau werd in de meeste onderzoeken een verminderde functionele netwerksterkte gevonden in relatie tot predisponerende risicofactoren. Studies naar precipiterende factoren lieten over het algemeen een verminderde efficiëntie van functionele netwerken zien. Tijdens delirium werden functionele hersennetwerken gekenmerkt door verminderde alfaband EEG-connectiviteitssterkte en verminderde fMRI-netwerkintegratie. Samenvattend vonden we aanwijzingen dat een minder verbonden en minder geïntegreerd hersennetwerk een onderliggend mechanisme zou kunnen zijn in de pathofysiologie van delirium.

Empirisch onderzoek naar het gezamenlijke effect van risicofactoren voor delirium op het functionele netwerk zouden kunnen helpen bij een uniform begrip van kwetsbaarheid voor delirium. Daarom hebben we in hoofdstuk 3 de hypothese geëvalueerd dat predisponerende risicofactoren voor delirium gerelateerd zijn aan soortgelijke neurofysiologische veranderingen als tijdens delirium. Deelnemers van 65 jaar en ouder $(N=206)$ ondergingen resting-state EEG-metingen en werden beoordeeld op predisponerende risicofactoren voor delirium, namelijk leeftijd, alcoholmisbruik, cognitieve problemen, depressie, functionele beperkingen, beroerte in de voorgeschiedenis en fysieke status. De EEG-kenmerken die bestudeerd werden waren relatieve deltapower, alfa-connectiviteitssterkte en netwerkintegratie. Functionele beperkingen bleken gerelateerd te zijn aan verminderde alfa-connectiviteit. Andere predisponerende risicofactoren voor delirium hadden geen effect op de bestudeerde EEG-kenmerken. Dit suggereert dat kwetsbaarheid voor delirium niet consistent gerelateerd is aan veranderingen in EEG-kenmerken zoals tijdens een delirium. In hoofdstuk 4 hebben we getest of predisponerende risicofactoren voor delirium gerelateerd zijn aan fMRI-netwerkveranderingen in een populatie van ouderen zonder delirium. In deze multicenter studie werden resting-state fMRI-scans geanalyseerd van 222 ouderen. Functionele connectiviteitssterkte, netwerkefficiëntie en netwerkintegratie werden 
bestudeerd, aangezien deze parameters in eerdere studies verstoord waren tijdens delirium. Predisponerende risicofactoren voor delirium bleken in deze studie niet gerelateerd te zijn aan delirium-gerelateerde fMRI-netwerkveranderingen. Een hogere leeftijd was, binnen ons cohort van ouderen, gerelateerd aan functionele connectiviteit, maar in de tegenovergestelde richting dan van tevoren voorspeld was. Delirium gerelateerde functionele netwerk verstoringen kunnen daarom niet worden beschouwd als het onderliggende mechanisme voor kwetsbaarheid voor delirium.

Het tweede gedeelte van dit proefschrift was gericht op het klinische syndroom van delirium zelf. Eerdere studies hebben met behulp van EEG laten zien dat delirium gerelateerd is aan verminderde functionele connectiviteit en verstoorde netwerkorganisatie. Daarnaast is in een $\mathrm{fMRI}$ studie tijdens delirium een verstoorde connectiviteit gevonden tussen twee specifieke hersenregio's die betrokken kunnen zijn bij cognitie, aandacht of bewustzijn, te weten tussen de posterior cingulate cortex en de dorsolaterale prefrontale cortex. In hoofdstuk 5 wilden we het begrip van de globale organisatie van het functionele netwerk tijdens het delirium vergroten en mogelijke veranderingen lokaliseren met behulp van fMRI Resting-state fMRI-scans van negen delirante patiënten, zeven post-delirium patiënten en dertien niet-delirante klinische controles werden geanalyseerd. Tijdens delirium werd een verminderde functionele netwerkefficiëntie en verminderde functionele netwerkintegratie gevonden. Bovendien was delirium gerelateerd aan het verlies van de hubfunctie in de rechter posterior cingulate cortex. De duur van het delirium was sterk gerelateerd aan verlies van functionele netwerkintegratie. Na delirium nam de connectiviteit af en werden complexe regionale veranderingen gevonden. Deze bevindingen tonen aan dat delirium gezien kan worden als desintegratie van functionele interacties tussen hersengebieden, en suggereren langer aanhoudende effecten in de hersenen, nadat het syndroom klinisch gezien is verholpen. Aangezien delirium een wisselend beloop heeft, is het aannemelijk dat de stoornis niet uitsluitend berust op een statisch concept. Het hersennetwerk is dynamisch en flexibel en herconfigureert continue, afhankelijk van de processen die plaats vinden. Cognitieve processen lijken (deels) afhankelijk te zijn van deze dynamisch manier van functioneren en de flexibiliteit van het hersennetwerk. In hoofdstuk 6 hebben we geëvalueerd of patiënten met delirium een verminderde flexibiliteit van de posterior cingulate cortex hebben vergeleken bij klinische controles. De flexibiliteit van de rechter en linker posterior cingulate cortex werd onderzocht bij 9 delirante patiënten en 13 klinische controles. Deze studie liet zien dat de flexibiliteit van de posterieure cingulate cortex niet verschilt tussen patiënten met delirium en klinische controles. Dit geeft aan dat verstoorde flexibiliteit mogelijk geen onderdeel is van de acute cognitieve problemen die tijdens delirium worden waargenomen.

Het laatste gedeelte van dit proefschrift was gericht op longitudinale veranderingen na delirium. Delirium gaat gepaard met een verhoogd risico op langdurige cognitieve problemen en dementie. Zowel tijdens postoperatief delirium als bij patiënten met dementie zijn verminderde connectiviteit en een verstoorde organisatie van het functionele hersennetwerk beschreven. In hoofdstuk 7 hebben we onderzocht of de ontwikkeling van postoperatief delirium gerelateerd is aan veranderingen van het functionele hersennetwerk over de tijd, in een populatie van ouderen die een operatie ondergaat. Patiënten die electieve chirurgie ondergingen, werden vóór en drie maanden na de operatie klinisch beoordeeld en kregen een resting-state $\mathrm{fMRI}$ scan. Delirium werd gemeten tijdens postoperatieve ziekenhuisopname. Globale fMRI connectiviteit, netwerkefficiëntie en netwerkintegratie werden geanalyseerd bij 246 patiënten, waarvan er 38 (16\%) postoperatief delirium ontwikkelden. Dit onderzoek liet zien dat delirium gerelateerd is aan een verminderde postoperatieve functionele connectiviteit, in deze populatie van ouderen die een operatie ondergaat, in tegenstelling tot een verhoogde postoperatieve functionele connectiviteit bij niet-delirante controles. Bovendien was de verminderde functionele connectiviteitssterkte gerelateerd aan cognitieve achteruitgang, onafhankelijk van het postoperatieve delirium. We hebben dan ook sterke vermoedens dat een verstoorde connectiviteit gerelateerd is aan het verhoogde risico op langdurige cognitieve problemen en dementie na delirium. 


\section{Dankwoord}

De afgelopen jaren heb ik van veel mensen hulp gekregen. Mede dankzij hen ligt hier nu een mooi eindresultaat. Ik wil hen hier graag enorm voor bedanken!

Geachte prof. dr. Slooter, beste Arjen, heel erg bedankt voor alle begeleiding tijdens mijn promotie en de mogelijkheden die je mij gegeven hebt. Je bent een uitstekende onderzoeker en bovenal een geweldig persoon, precies het voorbeeld dat ik tijdens mijn promotie nodig had. Jouw enthousiasme en bevlogenheid zijn ongekend. Je creëert voor de onderzoekers in jouw onderzoeksgroep een hele prettige werksfeer en roept op om, naast hard werken, ook van het leven te genieten. Dit heb ik zeer gewaardeerd. Onze overleggen waren altijd inspirerend. Je zorgde ervoor de we ons, ondanks de soms uitdagende omstandigheden binnen de BioCog studie, niet van de wijs lieten brengen. Een mooi boekje zou er komen, en zie hier, dat is dankzij gezamenlijke inspanningen ook gelukt. Ook naast de onderzoeksvaardigheden heb ik veel van je geleerd, zoals dat je naar een werktrip altijd hardloopspullen mee moet nemen, dat je een vreemde stad het beste per fiets kan ontdekken, en dat je nooit weet wat je aan kan treffen in een museum voor moderne kunst. Ontzettend bedankt, ik zal onze samenwerking missen.

Geachte prof. dr. Hendrikse, beste Jeroen, veel dank voor de begeleiding tijdens mijn promotie en alle teamspirit. Misschien waren we niet het enige 'dreamteam', maar dat hinderde niet. Deze term en ookjouw humor zorgde voor veel positiviteit, die we tijdens het uitdagende BioCog project goed konden gebruiken. Ook de schrijfdagen en de presentatie cursus waren erg waardevol tijdens mijn promotie. Jouw persoonlijke manier van leidinggeven is zeer inspirerend.

Geachte dr. van Dellen, beste Edwin, wat ben ik blij dat jij mijn copromotor was. Ondanks onze meestal tegenovergestelde agenda's wist je altijd een mogelijkheid te vinden voor overleg. Al bellend in de auto, via FaceTime vanuit Australië of op je vrije dag met een vrolijke prinses op de achtergrond. Wat de omstandigheden ook waren, je nam echt de tijd voor me. Grote problemen bleken meestal toch erg mee te vallen, ingewikkelde netwerktheorieën wist jij eenvoudig aan mij over te brengen en ik stond na ons gesprek altijd weer te poppelen om aan de slag te gaan met analyses of manuscripten. Je scherpe geest, je prachtige schrijfstijl, je gave om kennis over te brengen en je eindeloze enthousiasme; ik heb er heel veel van geleerd. Veel dank voor alle begeleiding, ik ga het missen!

Geachte prof. dr. Cahn, beste Wiepke, veel dank voor de geweldige stageplek bij de GROUP studie tijdens mijn master Neuroscience \& Cognition. Hier werd de passie voor grote patiëntgebonden studies bij mij aangewakkerd. Mede dankzij deze mooie ervaring ben ik aan mijn promotietraject begonnen. Ik waardeer het heel erg dat je deel uitmaakt van mijn lees- en promotiecommissie.

Geachte leden van de lees- en promotiecommissie, hartelijk dank voor het beoordelen van mijn proefschrift en de verdediging.

Alle deelnemers van de BioCog studie, heel erg bedankt voor uw tijd en deelname aan het onderzoek. Dankzij u was deze geweldige studie mogelijk.

Dear prof. dr. Winterer, thank you for initiating the BioCog study and the opportunity to collaborate with you.

Dear prof. dr. Spies, thank you for the great collaboration during the BioCog study, and later on during the CLEO study.

Beste medewerkers van het trialbureau van de intensive care, dank voor alle hulp! Sandra, Ada, Gea, Margereth, Joanne, José, jullie stonden altijd voor ons klaar om patiënten te informeren over de studie, te screenen op delirium, of om bloedafnames te doen.

Beste medewerkers van het trialbureau van de radiologie, bedankt voor het inplannen van alle honderden MRI scans van de BioCog deelnemers en het meedenken over de logistiek. 
Beste Niels, wat ontzettend fijn dat jij vanaf dag één bij de BioCog studie betrokken was. Bedankt voor alle test-scans, het mogelijk maken van onze EEG-MRI opstelling, jouw uitleg van ons uitdagende protocol aan de andere laboranten en de gezelligheid. Met jou erbij kan er eigenlijk niets mis gaan. Zelfs toen we met pech langs de A1 stonden, konden we dankzij jouw hulp al gauw weer verder rijden.

Beste MRI laboranten, bedankt voor het scannen van alle BioCog patiënten Extra dank voor al het geduld, de flexibiliteit, het extra schoonmaken nadat de patiënten met een plakkerige EEG cap in de scanner (en in de stoel, kleedruimte en wasbak) waren geweest en de goede gesprekken tijdens het scannen.

Beste Eline, en later Vincent, veel dank voor de hulp, met een grote glimlach, bij al het papierwerk wat bij onderzoek komt kijken en alle UMC kennis. Beste Christine en Rosalie, veel dank voor het meedenken over de BioCog logistiek, de altijd opbeurende gesprekjes en het fungeren als 'levende wegwijzer' naar E2.

Beste Judith en POS medewerkers, veel dank dat we via de preoperative screenings (POS) poli de patiënten voor de BioCog studie mochten benaderen.

Alle personen die direct of indirect hebben bijgedragen aan de BioCog studie in het UMC Utrecht, ontzettend bedankt! Een samenwerking tussen de intensive care, anesthesiologie, radiologie, psychiatrie en zeven chirurgische afdelingen om een studie te kunnen uitvoeren is heel bijzonder en waardevol.

Alle co-auteurs, bedankt voor de goede samenwerking en jullie bijdrage aan de hoofdstukken van mijn proefschrift.

Beste prof. dr. Stam en KNF onderzoeksgroep van het VUmc, in het bijzonder Arjan, Ida, Meenakshi en Lennard, veel dank dat ik tijdens een groot deel van mijn promotie kon aansluiten bij jullie interessante onderzoeksoverleggen. De kennis in jullie onderzoeksgroep is enorm en ik heb heel veel van jullie geleerd. De goede sfeer zorgde ervoor dat ik me erg welkom voelde.

Dear prof. dr. Kühn, thank you for the wonderful time in Hamburg. It was very inspiring to be part of your research group for several months. Dear Caroline, thank you for all your help with the graph analyses and all your inside tips about Hamburg.

Dear BioCog team of Charité hospital in Berlin, thank you for the warm collaboration. Anika, Fatima, Alissa, Kwaku, Kathrin, Norman, Susanne, Konstanze, Gunnar, Daniel, Rudolf, Friedrich, Saya, I am very glad that I got to know you in the past years. It was great working with all of you. I will definitely never forget our Jaime I adventures in Mallorca.

Datamanagers Wietze en Jacqueline zonder jullie zou de BioCog studie niet mogelijk geweest zijn. Bedankt voor de logistieke database die jullie voor ons gebouwd hebben. Hierdoor wisten we precies welke patiënt wanneer en waarvoor gebeld moest worden. Ook veel dank voor de hulp bij de dataextracties en het overzichtelijk maken van het totale BioCog databestand.

Lieve collega-onderzoekers van de psychiatrie in het UMC Utrecht, bedankt voor de interessante onderzoeksoverleggen, de journal clubs en de schrijfdagen die we samen organiseerden. Maya, mede netwerkonderzoeker, fijn om in dit ingewikkelde en snel-veranderende onderzoeksveld samen dingen uit te kunnen zoeken. Ik ben heel blij dat ik je heb leren kennen! Naast alle gezellige koffies, etentjes en cursussen samen, hebben we ook een onvergetelijke reis gemaakt naar Maleisië en Singapore voor het HBM congres. Nu je in Den Haag woont, blijven we elkaar in de toekomst zeker zien! Meenakshi, we liepen zo mooi gelijk, maar uiteindelijk was jij toch 1,5 jaar eerder klaar dan ik. De daadkracht waarmee jij door het leven gaat is erg inspirerend. De koffies, schrijfdagen, borrels en etentjes samen waren vaak precies wat ik nodig had om weer met goede moed verder te gaan. We houden zeker contact! Jessica, ooit mijn stagebegeleider bij de GROUP studie, inmiddels een goede vriendin. Ik kan altijd alles een beetje van je afkijken (zowel qua werk als privé) en dat is heel erg fijn! Bedankt voor alle leuke koffiemomenten in het UMC en alle gezelligheid daarbuiten, ook met 
Toon, Ramone en de kindjes. Ik kan niet wachten om straks jullie nieuwe aanwinst te mogen bewonderen!

Lieve Ilse, de beste partner-in-crime die ik me heb kunnen wensen Toen we eind 2014 samen in het Douwe Egberts café zaten voor een kennismakingsgesprek, hadden we geen idee wat ons te wachten zou staan. Gelukkig bleek al gauw dat we uit hetzelfde hout gesneden waren. Een paar keer per week om 7.30u in het UMC zijn om voorbereidingen te treffen voor dagen vol metingen; geen probleem! Dagjes heen en weer naar Berlijn voor overleg met onze BioCog collega's; geen probleem! Tientallen studenten begeleiden; ook geen probleem! Elke zoveelste inclusie vieren met taart, de vele borrels en etentjes, de wall of fame, het gelach dat je al op afstand vanuit onze kamer kon horen komen, de vlogs... we hebben er wat moois van gemaakt! In de afgelopen jaren is er een bijzondere vriendschap ontstaan en ik weet zeker dat we die in de toekomst voort zullen zetten. Ook met de grote en kleine mannen erbij. Bedankt voor alles! Ik ben heel blij dat je (zelfs tijdens je verlof!) mijn paranimf wilt zijn.

Lieve collega-onderzoekers van F6: Thomas, Marlies, Kirsten, Diana, Ivo Jos, Martine, Mathilde, bedankt voor de verbreding, het meedenken en de nodige afleiding. In het bijzonder Lisette, zonder jou zou ik mijn laatste studie nooit op tijd afgekregen hebben. Het bijna dagelijks bellen tijdens de corona lockdown periode, zorgde dat ik de laatste hordes van mijn promotietraject met een stuk meer plezier kon nemen. Heel erg bedankt hiervoor

Lieve E2 collega's veel dank voor de fijne werksfeer, de goede gesprekken, de lunches en borrels. Ik ga jullie missen! Tianne, zo'n goede onderzoeker en dan toch zo bescheiden. Bedankt voor alles wat ik van je heb mogen leren en voor alle gezelligheid. Met als toppunt natuurlijk de trip naar Vilamoura voor de EDA, waar we na het harde werken konden ronddobberen in ons eigen zwembad en met ons huurautootje de buurt konden verkennen (oppassen geblazen voor pensionado's in golfkarretjes). Lotte, award winnaar van 'beste collega'! Jij verspreidt de leukste handgeschreven briefjes, legt chocoladerepen klaar op precies het goede moment en hebt warme connecties met de paashaas en de pieten. Van jou vind je geen tweede!
Ellis, ik ben zo blij dat ik jou heb leren kennen. Waar jij bent is het feest. Als wij beginnen te praten is er zo een uur voorbij en dan zijn we zeker nog niet uitgepraat. Bedankt voor de fijne vriendschap de afgelopen jaren! Wietze, bij jou voelt iedere F6 of E2-er zich op zijn gemak. Je neemt de tijd om te luisteren en helpt iedereen. Het is super dat jij er bent! Ellen, je hebt ons ontzettend geholpen met jouw werk voor de BioCog studie. Toen wij inmiddels honderden patiënten gescand hadden, kon jij het van ons overnemen. Het was fijn om jouw humor, rust en gezelligheid erbij te hebben. Ook Sandra, Suzanne en Michel, bedankt! Het was heel leuk om de laatste maanden met jullie een kamer te delen.

BioCog stagiaires, de helden van dit project! Zo'n grote studie, die 24 uur en 7 dagen per week door ging, met zo veel metingen en zo veel telefoontjes, kon niet bestaan zonder jullie grandioze inzet. Heel erg bedankt voor jullie enthousiasme, levenslust, altijd vriendelijke omgang met de patiënten, geduld bij het EEG-cap plakken, goede ideeën en gezelligheid. Emily, Lieke, Amtul, Dorian, Susan, Merel, Prescilla, Corinne, Beatrijs, Rutger Ellen, Rianne, Aletta, Yarit, Ilona, Raoul, Willem-Jan, Rebecca, Michel, Luna, Myriam, Daan, Joyce, Rosa, Noam, Carla, Lara, Mariëlle, Fienke. En natuurlijk ook aan Babeth, Jolien en de weekendstudenten veel dank!

Lief CLEO-team: Rutger, Annemarie, Arjen, Pauline, Ineke, Tjeerd, Ilse Fienke. Bedankt voor de gezellige (soms dagenlange) overleggen, de energie, de telkens nieuwe invalshoeken en de lastige uitdagingen die dan toch tot een goed einde werden gebracht. Door het werken aan het CLEO project kreeg ik precies het goede evenwicht dat ik nodig had om me tijdens mijn 'promotiedagen' te kunnen focussen op analyseren en schrijven. Ik ga jullie missen!

Lieve vrienden en vriendinnen, heel erg bedankt voor alle support en voor de nodige afleiding in de afgelopen jaren. Jullie zijn erg waardevol voor mij. Een aantal mensen wil ik graag in het bijzonder bedanken.

Lieve Tamar, Jolien en Noor, oud master-genootjes, bedankt voor de gezellige koffiemomenten in het UMC, het delen van de PhD struggles 
(Tamar), de geweldige hulp bij het invoeren van de BioCog data in een onmogelijk systeem (Jolien) en de gesprekken over de toekomst.

Lieve Harr, al sinds groep 4 vriendinnen, wat is dat fijn. De leukste dingen hebben we sinds die tijd samen gedaan. Samen naar de middelbare school, studeren en wonen in Amsterdam, reizen, feestjes, croissantjes en taartjes. Met jou kan ik alles bespreken en problemen verdwijnen dan als sneeuw voor de zon. Bedankt voor alle support de afgelopen jaren!

Lieve Romy, heel erg bedankt dat je mijn paranimf bent! Wie had dat nou gedacht toen we samen stage liepen in het Curium, inmiddels alweer meer dan zes jaar geleden. We begrijpen elkaar zo goed en hebben zo'n bijzondere en fijne vriendschap. Vaak hoefik maar een paar woorden te zeggen en weet je al precies wat ik bedoel. Met jou aan mijn zijde heb ik alle vertrouwen in de verdediging, en in de toekomst.

Lieve Myrte, Maria, Tess, Lindy, Jantien en Letty, wat zou ik zonder jullie zijn! Af en toe verlang ik ernaar om de tijd even terug te draaien. Om weer samen met jullie in de collegebanken te zitten, daarna samen te koken, te eten en te dansen. Die mooie tijd vormde de basis voor een hechte vriendschap, die de afgelopen jaren alleen maar sterker geworden is. Met jullie speculeren, wereldproblemen oplossen en bedrijven oprichten, is voor mij pure ontspanning. Bedankt voor de leuke etentjes, koffies en theetjes, reizen, feestjes, en sinds kort ook 'Houseparty' sessies! Want gedeeld door zeven is alles weinig, behalve de liefde!

Lieve Sabine, ik weet niet waar de clichés over schoonmoeders vandaan komen, maar ik kan me er gelukkig niet in vinden. Heel lief dat je het altijd over 'mijn kids' hebt wanneer je Ramone en mij bedoelt. Bedankt voor alle warmte en gezelligheid, ook tijdens de Duits/Engels/Nederlandse (werk)trip naar Mallorca.

Lieve Ruben en Jonathan, leukste en liefste broertjes van de wereld! Qua werk zijn we alledrie erg uiteenlopend terecht gekomen, van ingenieur, tot sportief pedagogisch medewerker, tot onderzoeker. Ik denk dat ik in de afgelopen jaren niet meer dan een paar zinnen metjullie over mijn onderzoek heb gewisseld. Met jullie is het altijd fijn en vertrouwd. Bedankt hiervoor! Gezellig dat Eline er sinds een tijdje ook bij is.

Lieve papa en mama, bedankt voor het stimuleren van mijn nieuwsgierige karakter. Tijdens jullie liefdevolle opvoeding mocht ik veel uitproberen. Hierdoor weet ik goed wat ik wil en wat ik kan. Jullie hebben me geleerd om door te zetten en altijd optimistisch te zijn. Twee eigenschappen die tijdens mijn promotie van essentieel belang bleken. Jullie zijn er altijd voor me en dat betekent erg veel. Ook veel dank voor het oppassen op Melchior waardoor ik met een gerust hart mijn promotie af kon ronden.

Liefste Ramone, bij jou ben ik echt thuis. Ik ben heel blij dat ik zo veel feestelijke avonturen metje mag beleven. Met het vrolijke snoepje Melchior erbij zijn we nog completer. Bedankt voor de stabiele basis, voor de honderden fietstochtjes in de kou, regen of zon, 's ochtends rond $7.00 \mathrm{u}$ naar Den Haag Centraal, voor alle perfecte oplossingen en voor alle liefde. Oja, en natuurlijk voor de mooie illustratie van mijn proefschrift. 


\section{About the author}

Simone was born in The Hague on November 24th, 1990. During high school (Christelijk Gymnasium Sorghvliet, The Hague) she met her husband Ramone (married June 2nd, 2017), and she graduated in 2008. Afterwards, she started her bachelor study, Biological Psychology at the University of Amsterdam, and graduated in 2012. She became fascinated with brain research and started the research

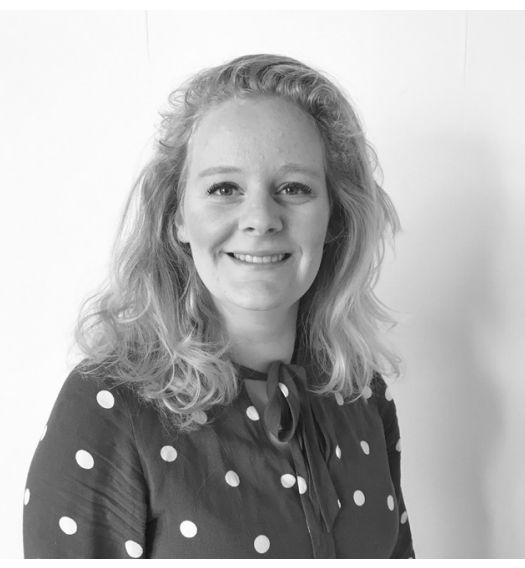

master study Neuroscience \& Cognition at Utrecht University. During her master's, she performed internships at the Psychiatry department of the University Medical Center Utrecht and at Curium LUMC, center for child and youth psychiatry, in Oegstgeest. She additionally worked as research assistant at the Psychiatry department of the University Medical Center Utrecht. She obtained her master's degree in 2014.

In 2015, she began her PhD research in the field of delirium at the Department of Intensive Care of the University Medical Center Utrecht, under supervision of prof. dr. A.J.C. Slooter, prof. dr. J. Hendrikse and dr. E van Dellen. She coordinated the BioCog study, a large multicenter study on delirium and postoperative cognitive impairment, and supervised research projects of over 20 bachelor's and master's students. In 2018 she started working as a postdoctoral researcher on a new multicenter study: the clinical evaluation of DeltaScan. She received the price for Best Oral Presentation at the European Delirium Association, Utrecht in 2018. After obtaining a network grant of ZonMw in 2019, she was able to visit the Psychiatry department of the Eppendorf Universitäts Klinikum of Hamburg for several months to learn new research skills and to expand her network. Shortly after her trip to Hamburg, she gave birth to her son Melchior (April 27th, 2019).
In May 2020, she started as Program Manager at ZonMw in The Hague. In her new role, she works on a program that enables researchers to develop new knowledge, in order to solve problems and challenges in health care. 


\section{List of publications}

This dissertation

1. van Montfort, SJT, Slooter, AJC, Kant, IMJ, Aarts, E, Vernooij, LM, Spies, CD, Hendrikse, $J$, van Dellen, E, on behalf of the BioCog consortium. Functional brain network changes after major surgery and delirium, submitted.

2. van Montfort, SJT, Slooter, AJC, Kant, IMJ, van der Leur, RR, Spies, CD, de Bresser, J, Witkamp, TD, Hendrikse, J, van Dellen, E, on behalf of the BioCog consortium. fMR network correlates of predisposing risk factors for delirium: a cross-sectional study, Neuroimage Clinical (2020), 27, 102347

3. van Montfort, SJT, van Dellen, E, Wattel, LL, Kant, IMJ, Numan, T, Stam, CJ, Slooter AJC, on behalf of the BioCog consortium. Predisposition for delirium and EEC characteristics, Clinical Neurophysiology (2020), 131, 1051 - 1058

4. van Montfort, SJT, van Dellen, E, Stam, CJ, Ahmad, A, Mentink, LJ, Kraan, CW, Zalesky, A, Slooter, AJC. Brain network disintegration as a final common pathway for delirium: a systematic review and qualitative meta-analysis, Neurolmage Clinical (2019), 23 101809.

5. van Montfort, SJT, Numan, T, van Dellen, E, Kyeong, S, Douw, L, Kim, JJ. Delirium is not associated with altered hub flexibility of the posterior cingulate cortex in an explorative fMRI pilot study, Clinical Neurophysiology (2018), 129, 2541 - 2543.

6. van Montfort, SJT, van Dellen, E, van den Bosch, AMR, Otte, WM, Schutte, MJL, Choi, SH, Chung, TS, Kyeong, S, Slooter, AJC, Kim, JJ. Resting state fMRI reveals network disintegration during delirium, Neurolmage Clinical (2018), 20, 35 - 41.

\section{Other}

1. Feinkohl, I, Borchers, F, Burkhardt, S, Krampe, H, Kraft, A, Speidel, S, Kant, IMJ, van Montfort, SJT, Aarts, E, Kruppa, J, Slooter, AJC, Winterer, G, Pischon, T, Spies, C. Stability of neuropsychological test performance in older adults serving as normative controls for a study on postoperative cognitive dysfunction. BMC Research Notes (2020), 13, 55.

2. Kant, IMJ, de Bresser, J, van Montfort, SJT, Slooter, AJC, Hendrikse, J. MRI markers of neurodegenerative and neurovascular changes in relation to postoperative delirium and postoperative cognitive decline. American Journal of Geriatric Psychiatry (2017), $25,1048-1061$
3. Kant, IMJ, de Bresser, J, van Montfort, SJT, Aarts, E, Verlaan, JJ, Zacharias, N, Winterer, G, Spies, C, Slooter, AJC, Hendrikse, J, on behalve of the BioCog consortium. The association between brain volume, cortical brain infarcts, and physical frailty, Neurobiology of Aging (2018), 70, 247 - 253.

4. Kant, IMJ, Mutsaerts, HJMM, van Montfort, SJT, Jaarsma-Coes, M, Witkamp, T, Zacharias, N, Winterer, G, Spies, C, Hendrikse, J, Slooter, A, de Bresser, J, on behalf of the BioCog consortium. The association between frailty and MRI features of cerebral small vessel disease, Scientific Reports (2019), 9, 11343

5. Lammers, F, Borchers, F, Feinkohl, I, Hendrikse, J, Kant, I, Kozma, P, Pischon, T, Slooter AJC, Spies, C, van Montfort, SJT, Zacharias, N, Zaborsky, L, Winterer, G, on behalf of the BioCog consortium. Basal forebrain cholinergic system volume is associated with general cognitive ability in the elderly. Neuropsychologica (2018), 119, 145 - 156.

6. Winterer, G, Androsova, G, Bender, O, Boraschi, D, Borchers, F, Dschietzig, TB, Feinkohl, J, Fletcher, P, Gallinat, J, Hadzidiakos, D, Haynes, JD, Heppner, F, Hetzer S, Hendrikse, J, Itterman, B, Kant, IMJ, Kraft, A, Krannich, A, Krause, R, Kühn, S, Lachmann, G, van Montfort, SJT, Müller, A, Nürnberg, P, Ofosu, K, Pietsch, M, Pischon, T, Preller, J, Renzulli, E, Scheurer, K, Schneider, R, Slooter, AJC, Spies, C, Stamatakis, E, Volk, HD, Weber, S, Wolf, A, Yürek, F, Zacharias, N. Personalized risk prediction of postoperative cognitive impairment - rationale for the EU-funded BioCog project. European Psychiatry (2018), 50, 34 - 39

7. van Montfort, SJT, Bos, DJ, Oranje, B, Durston, S, Smeets, PAM. The effects of omega3 polyunsaturated fatty acids on human brain morphology and function: what is the evidence? European Neuropsychopharmacology (2016), 26, 546 - 561

8. van Montfort, SJT, Maat, A, de Nijs, J, Derks, EM, Kahn, RS, Linszen, DH, van Os, J, Wiersma, D, Bruggeman, R, Cahn, W, de Haan, L, Krabbendam, L, Myin-Germeys, I, GOUP investigators. Emotion processing in schizophrenia patients is state and trait dependent, Schizophrenia Research (2015), 161, 292 - 298. 
